Tuning the electronic properties of two-dimensional systems

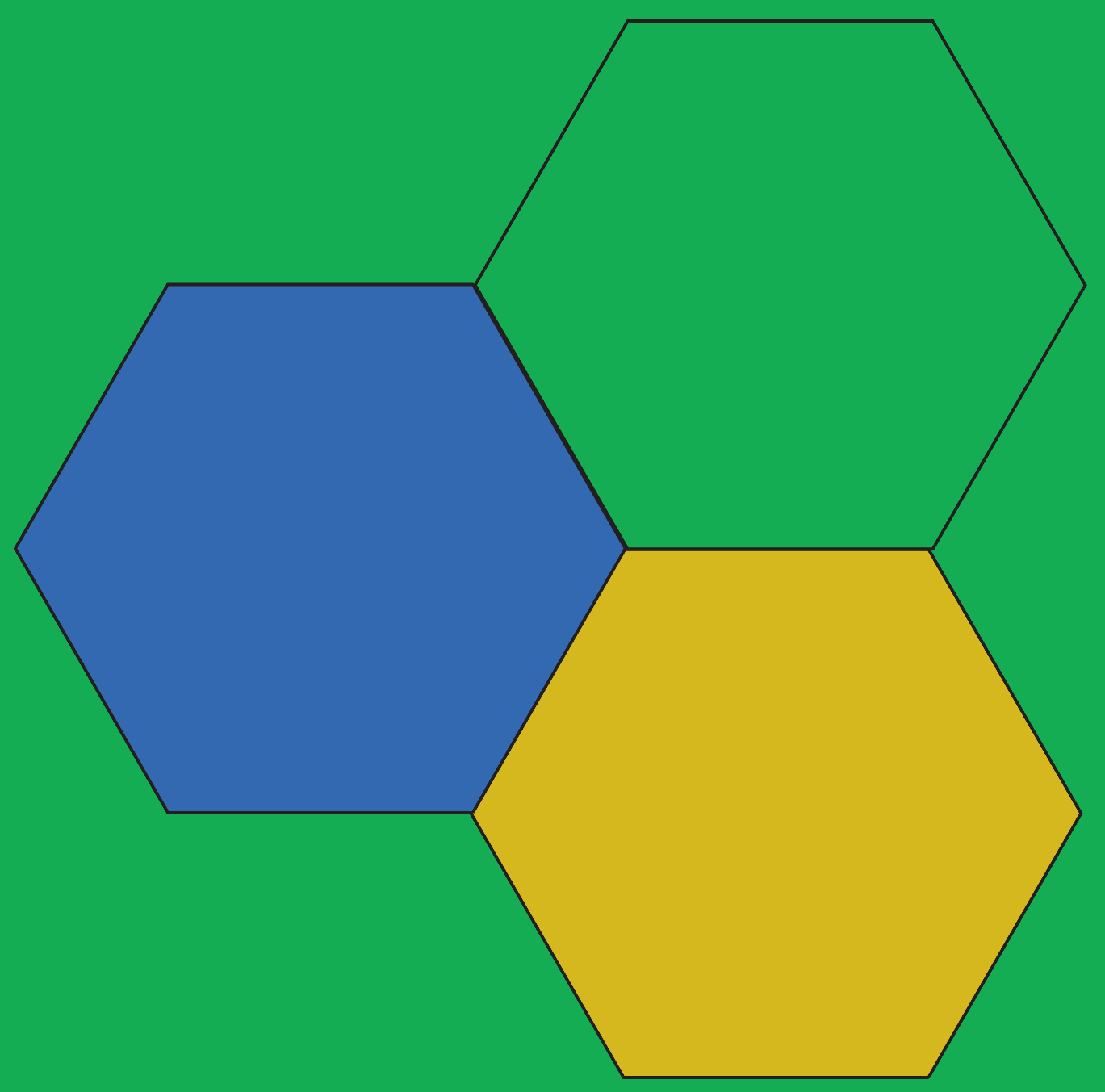

Yuqiang Gao 


\section{TUNING THE ELECTRONIC PROPERTIES OF TWO-DIMENSIONAL SYSTEMS}

Yuqiang Gao 
This dissertation has been approved by:

Supervisor

Prof. dr. P. J. Kelly (promotor)

Co-supervisor

Prof. dr. G.H.L.A. Brocks

Cover design: Yuqiang Gao

Printed by: Ipskamp printing, Enschede, The Netherlands

ISBN: 978-90-365-5005-5

DOI: $10.3990 / 1.9789036550055$

(C)2020 Yuqiang Gao, The Netherlands. All rights reserved. No parts of this thesis may be reproduced, stored in a retrieval system or transmitted in any form or by any means without permission of the author. Alle rechten voorbehouden. Niets uit deze uitgave mag worden vermenigvuldigd, in enige vorm of op enige wijze, zonder voorafgaande schriftelijke toestemming van de auteur. 


\title{
TUNING THE ELECTRONIC PROPERTIES OF TWO-DIMENSIONAL SYSTEMS
}

\author{
DISSERTATION \\ to obtain \\ the degree of doctor at the Universiteit Twente, \\ on the authority of the rector magnificus, \\ Prof. dr. T.T.M. Palstra, \\ on account of the decision of the Doctorate Board \\ to be publicly defended \\ on Wednesday 27 May 2020 at 16:45 uur
}

by

Yuqiang Gao

born on 5 April, 1991

in Anhui, China 
Graduation Committee

Chairman/secretary: Prof. dr. J. L. Herek University of Twente

Supervisor:

Prof. dr. P. J. Kelly

University of Twente

Co-supervisor:

Prof. dr. G.H.L.A. Brocks University of Twente

Committee Members: Prof. dr. ir. G. Koster

University of Twente

Prof. dr. C. Filippi

University of Twente

Prof. dr. T. Dietl

Polish Academy of Sciences

Dr. E. Santos

Queen's University Belfast

Dr. J. Ye

University of Groningen 


\section{Contents}

1 Introduction 1

1.1 Materials investigated in the thesis $\ldots \ldots \ldots \ldots . \ldots \ldots$

1.1.1 2D-Xenes $(\mathrm{X}=\mathrm{Si}, \mathrm{Ge}, \mathrm{Sn}) \ldots \ldots \ldots . \ldots . \ldots 2$

1.1.2 Transition metal dichalcogenide (TMD) monolayers . . . . . 2

1.1.3 Two-dimensional electron gas at $\mathrm{LaAlO}_{3} / \mathrm{SrTiO}_{3}$ interface . . 3

1.2 Method .................... . 4

1.2.1 Density Functional Theory . . . . . . . . . . . . . 4

1.2.2 Exchange and Correlation Potential . . . . . . . . . . 5

1.2.3 Basis Set: Plane-Waves . . . . . . . . . . . . . . . 5

1.2.4 Maximally Localized Wannier Function . . . . . . . . . . . 6

1.2 .5 Modelling impurity states . . . . . . . . . . . . . 7

1.3 Outline of this thesis . . . . . . . . . . . . 8

2 The ferroelectric field effect on the 2DEG at $\mathrm{LaAlO}_{3} / \mathrm{SrTiO}_{3}(001)$ interface 9

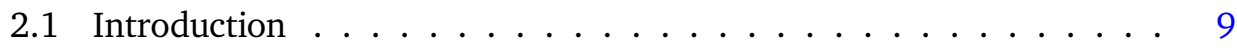

2.2 Computational Method . . . . . . . . . . . . . . . . 11

2.3 Results and Discussion . . . . . . . . . . . . . . . . . . 12

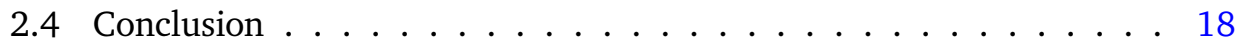

3 High temperature itinerant ferromagnetism in p-doped monolayers of $\begin{array}{ll}\mathrm{MoS}_{2} & 21\end{array}$

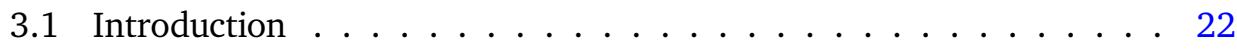

3.2 Computational Details . . . . . . . . . . . . . . . . 25

3.3 Results . . . . . . . . . . . . . . . . . . . 27

3.3.1 Single impurity limit: $\mathrm{V}$ in $\mathrm{MoS}_{2} \ldots \ldots \ldots . \ldots 29$

3.3.2 Binding of $\mathrm{V}$ impurity pairs . . . . . . . . . . . . . 40

3.3.3 Magnetic Interaction of impurity pairs . . . . . . . . . . 42

3.3.4 Nb and Ta in $\mathrm{MoS}_{2} \ldots \ldots \ldots \ldots$. . . . . . . . . . . . . . . . . . . . . 47

3.4 Magnetic Ordering . . . . . . . . . . . . . . . . . . . . . . . . . . 49

3.4.1 Single ion anisotropy . . . . . . . . . . . . . . . . 49

3.4.2 Monte Carlo calculations . . . . . . . . . . . . . . 53

3.4 .3 Curie temperature . . . . . . . . . . . . . . 54 
3.5 Comparison with other work $\ldots \ldots \ldots \ldots$

3.6 Discussion . . . . . . . . . . . . . . . . 61

3.7 Summary \& Conclusions . . . . . . . . . . . . . . . . . . . . . 63

3.8 Appendices . . . . . . . . . . . . . . . . . . . . . 64

3.8.1 Sulphur reference atom . . . . . . . . . . . . . 64

3.8 .2 Hydrogen atom in LDA/GGA . . . . . . . . . . . . . 64

4 Systematic DFT study of structural \& electronic properties in $p$-doped $\begin{array}{ll}\mathrm{MX}_{2} \text { ML } & 67\end{array}$

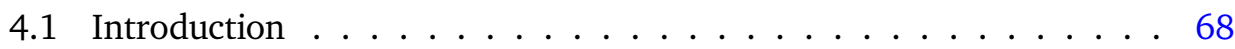

4.2 Computational Method . . . . . . . . . . . . . . 71

4.3 Results . . . . . . . . . . . . . . . . . 72

4.3.1 $\mathrm{MX}_{2}$ Monolayers . . . . . . . . . . . . . . . . 72

4.3.2 Structural and electronic properties of p-doped $\mathrm{MX}_{2}$ monolayers . . . . . . . . . . . . . 73

4.3.3 Single ion anisotropy $\ldots \ldots \ldots$. . . . . . . . 81

4.3.4 Interaction with intrinsic defects . . . . . . . . . . 85

4.3.5 Electron doping: Rhenium . . . . . . . . . . . . 88

4.4 Discussion . . . . . . . . . . . . . . . . . . . . . . . . . 89

4.5 Conclusions . . . . . . . . . . . . . . . . . 91

5 Edge reconstruction of 2D-Xene (X=Si, Ge, Sn) nanoribbons 93

5.1 Introduction . . . . . . . . . . . . . . 93

5.2 Computational Method . . . . . . . . . . . . . . . . . 95

5.3 Electronic properties . . . . . . . . . . . . . . . . . . . 96

5.4 Edge reconstruction $\ldots \ldots \ldots$. . . . . . . . . . . . 97

5.5 Tight binding model . . . . . . . . . . . . . . . . . . . . . 99

5.6 Discussion . . . . . . . . . . . . . . . . . . . . . . 101

5.7 Conclusion . . . . . . . . . . . . . . . . . . 102

$\begin{array}{ll}\text { Bibliography } & 103\end{array}$

$\begin{array}{ll}\text { List of publications } & 119\end{array}$

$\begin{array}{ll}\text { Summary } & 121\end{array}$

$\begin{array}{ll}\text { Acknowledgements } & 123\end{array}$ 


\section{1 \\ Introduction}

Nanotechnology is about making electronic devices with dimensions of the order of 1-100 nanometers. The smaller the size, the greater the integration and the more powerful the circuits. However, as the dimensions are reduced, the problems associated with heat dissipation and quantum size effect become more prominent that limiting the further miniaturization of electronic devices. New kinds of materials might make an important contribution. In 2004, the preparation of graphene [1], a single carbon atom nano sheet, opened a new area of two-dimensional (2D) materials and attracted huge attention for its potential in the miniaturization of electronic devices. The spectacular properties that do not exist in their 3D counterparts $[2 ; 3 ; 4]$ also provide opportunities for the design of novel electronic devices. In addition, 2D materials offer great flexibility in term of tuning their electronic properties using electric fields [5], functionalization [6], doping [7], and structural tuning [8;9].

Quantum physics offers a deep understanding of materials at the atomic level. In principle, starting from quantum physics we can reproduce experimental results by solving the many body Schrödinger equation with only natural contents as input. Such calculations are called ab initio or first principles calculations. With the development of advanced methods and powerful supercomputers, ab initio calculations can describe the behaviour of electrons in materials. This has become an important method to interpret and predict new phenomena in materials science, especially for nanoscale systems, where quantum effects become more prominent.

In this thesis, I use first-principles density functional theory to investigate the electronic and magnetic properties of a number of two-dimensional systems. In the following, I will introduce the two-dimensional systems investigated in this thesis, 
the method used in the study, and outline of the thesis.

\subsection{Materials investigated in the thesis}

\subsubsection{D-Xenes $(\mathrm{X}=\mathrm{Si}, \mathrm{Ge}, \mathrm{Sn})$}

(a)

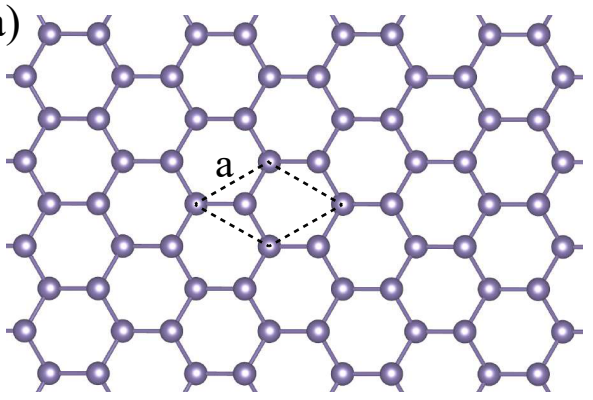

(b)

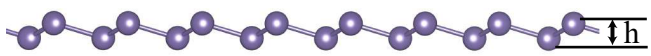

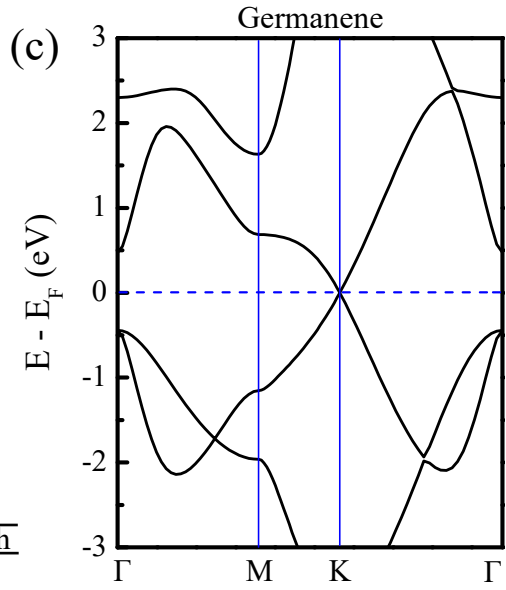

Figure 1.1: The atomic structure (a) top view and (b) side view and (c) band structure of hexagonal germanene.

By analogy with graphene, $2 \mathrm{D}$-Xenes $(\mathrm{X}=\mathrm{Si}, \mathrm{Ge}, \mathrm{Sn})$ consist of single layers of group IVA atoms arranged on a hexagonal lattice, for example germanene shown in Fig. 1.1a. Unlike graphene where atoms all lie in a plane, germanene has a buckled structure (Fig. 1.1b). The breaking of reflection symmetry in the direction perpendicular to the germanene layer makes the $p_{z}$ orbitals hybridize with the neighbouring $s p^{2}$ orbitals, resulting in a mixed $s p^{2}-s p^{3}$ hybridization, which stabilizes a corrugated hexagonal arrangement. Due to the symmetry of the honeycomb lattice, the band structure (Fig. 1.1c) shows a Dirac cone at the Fermi level similar to graphene with two linear bands crossing at $K / K^{\prime}$ points. However, for the large spin-orbit coupling $\xi$ in germanene, the dispersion relation become nonlinear: $E(k)= \pm \sqrt{\left(v_{F} k\right)^{2}+\xi^{2}}$, resulting in a massive Dirac cone and a band gap [10].

\subsubsection{Transition metal dichalcogenide (TMD) monolayers}

In order to be used as a building element for electronic applications, a band gap is needed. The absence of a band gap in graphene limits its potential for wide application in electronic industry. Transition metal dichalcogenide (TMDs) monolayers with the type $\mathrm{MX}_{2}(\mathrm{M}=\mathrm{Cr}, \mathrm{Mo}, \mathrm{W} ; \mathrm{X}=\mathrm{S}$, Se, Te) provide a promising alternative to 
gapless graphene. A monolayer of $\mathrm{MX}_{2}$ consists of a hexagonal plane of $\mathrm{X}$ atoms on either side of a hexagonal plane of $\mathrm{M}$ atoms as shown in Fig. 1.2a, in which the hybridization of X-p orbitals and M-d orbitals leads to a direct band gap ranging from 1-2 eV [11] (Fig. 1.2b).

(a)
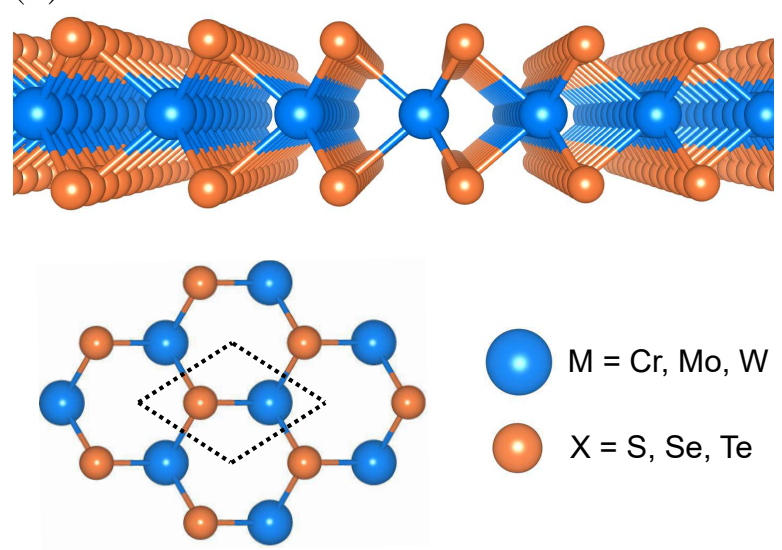

(b)

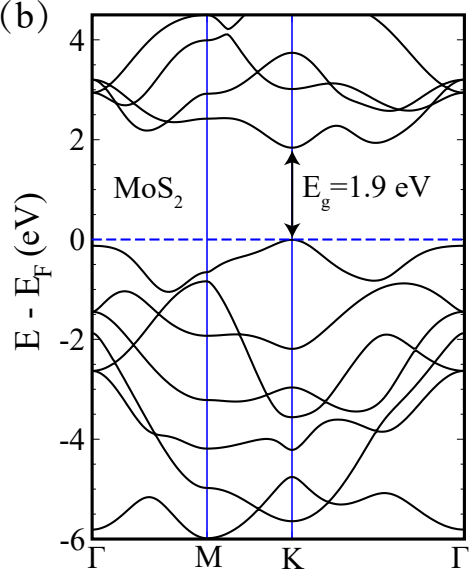

Figure 1.2: (a) The atomic structure of a monolayer of $\mathrm{MX}_{2}$ and (b) bandstructure of monolayer $\mathrm{MoS}_{2}$.

The weak van der Waals force in TMDs makes it possible to exfoliate monolayers of TMDs experimentally. The lack of inversion symmetry in monolayers of $\mathrm{MX}_{2}$ enhances spin orbit coupling (SOC) and breaks the Kramers degeneracy $\left(\epsilon_{\uparrow}(k)=\right.$ $\left.\epsilon_{\downarrow}(k)\right)$ in most of the Brillouin zone. Because of the time-reversal symmetry $\left(\epsilon_{\uparrow}(k)=\right.$ $\epsilon_{\downarrow}(-k)$ ), the splitting at $K$ and $K^{\prime}$ points is opposite. The SOC splitting in the valence band has a value of 0.1-0.4 eV, which is much higher than that in the conduction band [12].

\subsubsection{Two-dimensional electron gas at $\mathrm{LaAlO}_{3} / \mathrm{SrTiO}_{3}$ interface}

The two-dimensional electron gas (2DEG) with high carrier mobility formed at the n-type interface between the two band insulators $\mathrm{LaAlO}_{3}(\mathrm{LAO})$ and $\mathrm{SrTiO}_{3}$ (STO) [13] has sparked much interest in the so-called polar discontinuity. The polar catastrophe at the LAO/STO interface [14] is believed to be the main reason for the formation of the 2DEG, in which a charge transfer from the surface of the $\mathrm{LAO}$ layer to the n-type $\mathrm{LaO}^{+} / \mathrm{SrO}_{2}^{-}$interface balances the polar discontinuity as indicated in Fig. 1.3. For practical applications, the 2DEG should be field-tunable in order to be able to make a transistor. The large dielectric constant of LAO and STO make it an ideal system for high-frequency transistors. 

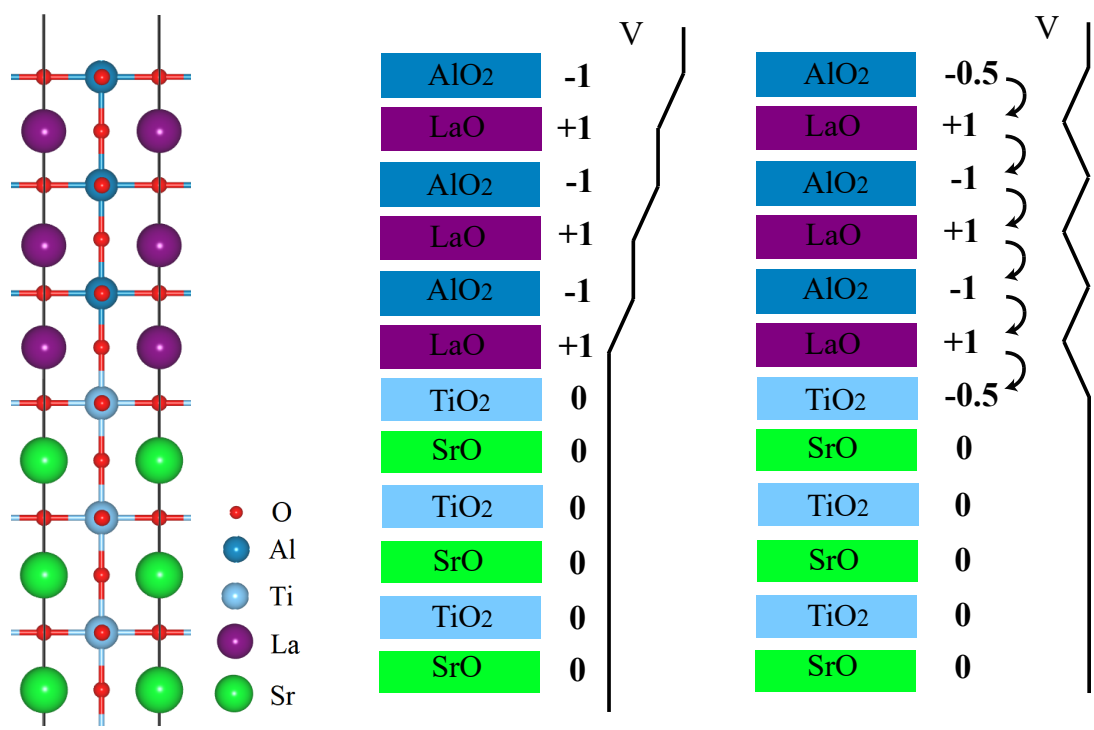

Figure 1.3: The atomic structure of n-type LAO/STO (001) interface and polar catastrophe model.

\subsection{Method}

\subsubsection{Density Functional Theory}

The electronic structure calculations performed in this thesis are based on density functional theory (DFT) developed by Kohn, Sham, and Hohenberg [15; 16]. DFT has proved very successful in calculating the electronic and structural properties of materials. The theory states that the ground state of an N-electron system is fully characterized by the total electron density $n(\boldsymbol{r})$ that depends on only three spatial coordinates. Any properties of the system can be expressed as a functional of the total electron density $n(\boldsymbol{r})$. According to the variational principle, a universal functional for the total energy, $E[n(\boldsymbol{r})]$, is minimized by the correct ground state electron density $n(\boldsymbol{r})$. The many body Schrödinger equations then can been solved explicitly with the universal function $E[n(\boldsymbol{r})]$. However, we do not have the exact form of the functional and normally a good approximation can give a reasonable accurate results. Kohn and Sham proposed the Kohn-Sham (KS) equation that makes solving many body Schrödinger equations possible with much less computational resources by reducing the many body problem of interacting electrons to a problem of non-interacting electrons moving in an effective potential. Non-interacting electron system means each individual electron moves independently of each other, only feeling the average electrostatic field from all the other electrons and nuclei. Hence the kinetic energy and Coulomb term can be calculated exactly in a noninteracting electron system. In the KS framework, apart from the Hartree potential 
that includes the non-interacting classic Coulomb interaction, the exchange and correlation potential account for the deviation from the exact potential.

\subsubsection{Exchange and Correlation Potential}

The development of different exchange-correlation potentials has been the main subject in computational material science for a long time. In general, they follow a Jacob's ladder according to the form of electron density functional. The most common one is the local density approximation (LDA), in which the inhomogeneous electron gas is approximated locally by a homogeneous electron gas with the same local density. Local spin density approximation (LSDA), the spin polarized variation, replaces the spin averaged energy density with the energy density for a polarized homogeneous electron gas. Improvement can be achieved by considering the gradient of charge density, namely the generalized gradient approximation (GGA). Hybrid functionals hybridize the approximated exchange-correlation potential with a portion of exact exchange from Hartree-Fock theory. The van der Waals force (dispersion) in layered materials like graphite comes from long-range, nonlocal electron correlation, which LDA and GGA neglect. To describe the van der Waals force properly, a non-local term should be added to the local correlation functional. This non-local term is like a Hartree potential but replacing the Coulomb kernel $\left(1 /\left|x-x^{\prime}\right|\right)$ with a van der Waals kernel $\left(1 /\left|x-x^{\prime}\right|^{6}\right)$.

\subsubsection{Basis Set: Plane-Waves}

To solve the KS equations, the KS orbitals are expanded in a basis set. Based on Bloch's theorem, the electron wavefunctions in a periodic potential are periodic in real space with a phase factor $e^{i k \cdot r}$. So the first choice of basis set for the wavefunctions corresponding to periodic potential are naturally plane waves, in which the periodic parts of the electron wavefunctions are expanded in plane waves. The kinetic energy and effective Coulomb potential are periodic in real space, they can be expanded in a plane wave basis simply by Fourier transformation. The kinetic operator becomes diagonal in the plane wave basis which greatly simplifies calculating the Hamiltonian.

The advantage of a plane wave basis is that it transforms a problem of calculating an infinite number of electronic wavefunctions in real space to that of calculating a finite number of electronic wavefunction in infinite number of $\mathrm{k}$ points in reciprocal space. The KS orbitals become $\psi_{n}^{k}$, where $\mathrm{n}$ is the index of KS orbitals and $\mathrm{k}$ ranges over the Brillouin zone. The problem can be further simplified by a finite k-point sampling, because the wavefunction at close $\mathrm{k}$ points are very similar. The reciprocal lattice vector $\mathrm{G}$ that expand $\mathrm{KS}$ orbitals into plane waves should be cut off at some point, such as $E_{c u t}=\frac{\hbar^{2}}{2 m}\left|\boldsymbol{k}+\boldsymbol{G}_{c u t}\right|^{2}$. Convergence tests should be done to get dense enough k-point sampling and cutoff energy. 
Problems arises on trying to expand the rapidly oscillating wavefunction near the nuclei where a huge number of plane waves is required. On the other hand, the valence electrons determine the chemical properties of the system, while the core electrons tightly bound to the nuclei make little contribution. For practical consideration, it is not necessary to accurately calculate the wavefunction in the core region. Instead, a smooth pseudopotential with the same scattering properties replaces the diverging real potential inside a core sphere near the nuclei. This allows the pseudo wavefunction to be expanded using much fewer plane waves. To guarantee the accuracy, the pseudopotential should be "norm conserving" in which the eigenenergies of pseudopotential are the same with that of real potential and the pseudo wavefunction should match the real wavefunction at and beyond the boundary of the core region.

In our electronic structure calculations, we use the Projector Augmented Wave (PAW) method, which guarantees high accuracies by keeping all-electron wavefunctions. It constructs the all-electron wavefunction from a pseudo wavefunction and atom-like functions localized near the nuclei. The pseudo wavefunction coincides with the all-electron wavefunction beyond the core region. Inside the core region, or called augmentation region, the wavefunction are rarely affected by the surrounding crystal and so can be constructed by the atom-like functions. The coefficients of the atomic orbitals come from expansion coefficients of the pseudo wavefunction inside the augmentation spheres.

\subsubsection{Maximally Localized Wannier Function}

Although in electronic calculations the extended Bloch orbitals $\psi_{n k}(r)$ are widely used, we have alternative representation in terms of localized Wannier function. Wannier functions can provide insights to the chemical bonding that are not easily obtained from Bloch functions. Because Bloch functions are periodic in real space and have a different phase for different $\mathrm{k}$. We can get a localized function (Wannier function) in real space by superposing the Bloch functions at different $\mathrm{k}$ points, which integrates over the Brillouin zone. To get the translational symmetry of Wannier function in real space, a phase factor $e^{-i \boldsymbol{k} \cdot \boldsymbol{R}}$ should be inserted into the integrand, where $\boldsymbol{R}$ is the real-space lattice vector. So actually the Wannier functions can be obtained by Fourier transformation of Bloch functions:

$$
w_{n R}(r)=\frac{1}{\sqrt{N}} \sum_{k} e^{-i k \cdot R} \psi_{n k}(r)
$$

in which $n$ is the band index and $\frac{1}{\sqrt{N}}$ is the normalization factor. Wannier functions form an orthogonal basis set. Each band $n$ has one Wannier function $w_{n}(r)$ localized at site $r$, which can be translated by a lattice vector $\boldsymbol{R}$ to generate replica Wannier functions $w_{n R}(r)$ in the entire periodic crystal. 
However, the transformation is not unique for the arbitrary phase of Bloch function at each $\mathrm{k}$ point. One localization procedure is needed to determine the maximally localized Wannier function, which can eliminate the nonuniqueness of Wannier functions and determine a constant phase for the Bloch function. For bands that are degenerate or crossing at certain $k$ points, Bloch states can be mixed via a unitary transformation $U_{m n}^{k}$ :

$$
\tilde{\psi}_{n \boldsymbol{k}}(r)=\sum_{m} U_{m n}^{k} \psi_{n \boldsymbol{k}}(r)
$$

The many body wavefunction is a Slater determinant, so a unitary transformation would not change the traces over the manifold bands. Marzari and Vanderbilt defined the quadratic spreads $\Omega$ of Wannier function as

$$
\Omega=\sum_{n}\left[\left\langle w_{n 0}\left|r^{2}\right| w_{n 0}\right\rangle-\left\langle w_{n 0}|\boldsymbol{r}| w_{n 0}\right\rangle^{2}\right]
$$

which gives a localization criterion for the Wannier function. Then the maximally localized Wannier function can be obtained by minimize the $\Omega$ with respect to the $U_{m n}^{k}$.

\subsubsection{Modelling impurity states}

Two chapters of this thesis are about modelling impurity states. So it is worth introducing how to model these states. Actually, since the appearance of quantum physics, people had started to apply it into modelling impurity states for the remarkable effect of the impurity on the properties of semiconductor. The understanding of donor and acceptor states in semiconductors contributes to the invention of the transistor which shapes modern society. In 1970s, to model these impurity states, the standard way was to use Green function method or effective mass theory (EMT). The impurity is considered to introduce an extra Coulomb potential at its site and localized impurity states in the forbidden energy gap. For strong and short range impurity potential, the deep in-gap states can be modelled using Green's functions, while the effective mass theory can capture the main characters of the weakly bound shallow impurity states. The Green function determines the potential self-consistently only in the region that significantly differ from that of unperturbed host. So the local environment of impurity states that are not perturbed can be described correctly. In EMT, the analogy of donor or acceptor to the hydrogen model makes the impurity states bound to the band extremes with an effective mass and screened Coulomb potential. The derivation of EMT with experiment results (called chemical shift) normally be corrected by the "central cell correction", in which in the "central cell" region the screening of impurity potential decreases by $\epsilon(q)$. 
Alternatively, we can model the impurity states in a supercell. The supercell is periodically repeated so that a periodic crystal is obtained. The size of the supercell should be large enough to minimize the interactions between impurity atoms in adjacent cells. The system with a few hundreds of atoms can be treated routinely. However, it can not describe a shallow system with impurity wavefunction extending over hundreds of lattice site. To describe the extended impurity potential well, the supercell should be very large which is beyond the computational capacity today. For the deep or not very shallow impurity states, supercell method would be very suitable. In this thesis, we would use supercell method to model the impurity states in monolayers of $\mathrm{MX}_{2}$.

\subsection{Outline of this thesis}

The thesis is structured as follows. In chapter 2, we study the ferroelectric field effect on the two-dimensional electron gas at the n-type LAO/STO (001) interface through a ferroelectric substrate $\mathrm{BaTiO}_{3}$ (BTO). We show that the carrier density at the LAO/STO interface can be reversely tuned by the ferroelectric field of the substrate. This originates from the ferroelectric field control of the intrinsic electric field in LAO.

In chapter 3, we explore the possibility of making a semiconducting monolayer of $\mathrm{MoS}_{2}$ ferromagnetic by introducing holes into the narrow Mo d band that forms the top of the valence band. By substitutionally doping group VB elements $(\mathrm{V}, \mathrm{Nb}, \mathrm{Ta})$ in monolayer of $\mathrm{MoS}_{2}$, we find the holes are fully polarized and pairs of such holes couple ferromagnetically unless the dopant atoms are too close. We analyse the mechanism behind the quenching and propose possible solutions to avoid the quenching by considering other TMD system such as monolayer of $\mathrm{MoSe}_{2}$ or $\mathrm{MoTe}_{2}$.

In chapter 4, we carry out a systematic study of the structural and electronic properties of single acceptor and double acceptor $(\mathrm{V}, \mathrm{Nb}, \mathrm{Ta}, \mathrm{Ti}, \mathrm{Zr}, \mathrm{Hf})$ doped $\mathrm{MX}_{2}$ monolayers in the single impurity limit, including impurity binding energy, structure distortion, and single ion magnetic anisotropy. The effect of intrinsic defects such as vacancy and antisite are discussed. The Curie temperature is evaluated in single acceptor doped monolayers of $\mathrm{MX}_{2}$.

In chapter 5, we study the structural reconstruction at the bare zigzag edge of 2D-Xene $(X=S i, G e, S n)$. An edge reconstruction with $3 a$ periodicity is predicted, which opens a gap and shows non-magnetic ground state. 


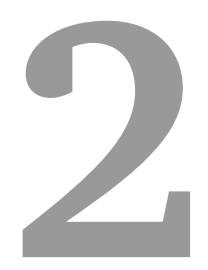

\section{The ferroelectric field effect on the two-dimensional electron gas at $\mathrm{LaAlO}_{3} / \mathrm{SrTiO}_{3}(001)$ interface *}

We perform first-principles calculations to explore the possibility of tuning the twodimensional electron gas at the $\mathrm{LaAlO}_{3} / \mathrm{SrTiO}_{3}$ (001) interface through $\mathrm{BaTiO}_{3}$ substrate. A metal-to-insulator transition is found at the interface as the polarization of $\mathrm{BaTiO}_{3}$ reverses. Through the potential analysis of the $\mathrm{LaAlO}_{3} / \mathrm{SrTiO}_{3} / \mathrm{BaTiO}_{3}$ superstructure, we find that the intrinsic electric field of LaAlO3 is significantly suppressed as the polarization points away from the $\mathrm{LaAlO}_{3} / \mathrm{SrTiO}_{3}$ interface, while it is enhanced with the polarization pointing to the interface. The ferroelectric field control of the intrinsic electric field, and therefore the electronic reconstructions at the interface, originating from the screening of polarization charges, opens the way to the development of novel nanoscale electronic devices.

\subsection{Introduction}

The (001) interface between two band insulators, $\mathrm{LaAlO}_{3}$ (LAO) and $\mathrm{SrTiO}_{3}(\mathrm{STO}$ ), which exhibits great variety of emergent phenomenon such as the two-dimensional electron gas (2DEG), superconductivity, magnetism, and their coexistence [13; 17; $18 ; 19 ; 20]$, has attracted great interests in searching new novel phenomenon

"published as: [Y.Q. Gao et al., Physica E: Low-dimensional Systems and Nanostructures 80, 2016, 195-201]. 
and manipulating these phenomenon at the interface. The 2DEG at the n-type $\mathrm{LAO} / \mathrm{STO}$ interface appears rather suddenly as the thickness of LAO layer reaches a critical value $\mathrm{d}_{c}=4$ unit cell (u.c.), while the interface is still insulating below $\mathrm{d}_{c}$ [21]. According to the widely acknowledged "polar catastrophe" model [13; 14], an electronic reconstruction will be induced by the polar discontinuity at the ntype $\mathrm{LaO}^{+} / \mathrm{TiO}_{2}^{0}$ interface, which transfers electrons from the surface $\mathrm{AlO}_{2}$ layer to the interface $\mathrm{TiO}_{2}$ layer to compensate the divergent electrostatic potential and leads to an conducting interface. The 2DEG formed at the LAO/STO interfaces has a large carrier density $\left(\sim 10^{13} \mathrm{~cm}^{-2}\right)$ and high carrier mobility $\left(\sim 10^{4} \mathrm{~cm}^{2} \mathrm{~V}^{-1} \mathrm{~s}^{-1}\right)$ $[13 ; 21 ; 22]$, which can be used as drain-source (DS) channels in field-effect transistor (FET).

In analogy to tuning the 2DEG in FET by gate field, many efforts have been done to manipulate the 2DEG at the LAO/STO interface through strain, electric field, modulation-doping, metal contact, surface treatment and etc $[21 ; 23 ; 24$; $25 ; 26 ; 27 ; 28 ; 29 ; 30 ; 31]$. However, strain and modulation-doping may induce unexpected structure disorder or electronic scattering, such as oxygen octahedral rotation [30], magnetism scattering [31], which leads to a relative small modulation of the carrier density of the 2DEGs. In contrast, electric field without perturbing the microstructure can be more efficient and controllable in tuning the carrier density of 2DEG $[21 ; 27 ; 32]$. A metal-insulator transition (MIT) accompanied with a memory behavior was observed at LAO/STO interface with $\mathrm{d}_{L A O}=3$ u.c. by applying a positive voltage to its back gate, in which the strong electric field of the 2DEG-defect sheet dipole layer is believed to stabilize the 2DEG at the interface [21]. For the samples with $\mathrm{d}_{L A O} \geq 4$ u.c., the conductivity can also be modulated by a gate voltage, although the MIT occurs only at low temperature [33]. Localization of 2DEG can also be induced by a polar phase transition in $\mathrm{SrTi}^{18} \mathrm{O}_{3}$ at negative gate voltage, which strongly reduces the lattice polarizability and the subsequent screening [27]. The ferroelectric phase transition in STO induced by electric field provides a new path to tune the 2DEG at the interface, in which the polarization partially screens or enhances the intrinsic electric field in LAO and thus changes the carrier concentration, although the mechanism behind the electric-field control of 2DEG is still under discussion [33; 34; 35].

Compared with polarizing the STO by a gate voltage, a ferroelectric substrate can be more efficient $[36 ; 37 ; 38]$. Recently, a giant and nonvolatile ferroelectric electroresistive diode was experimentally investigated by integrating a vertical ferroelectric capacitor into 2DEG at the interface. This indicates the ferroelectric field effect on the intrinsic electric field in LAO [39]. Meanwhile, the prediction of a 2DEG and a two-dimensional hole gas (2DHG) at the interface of $\mathrm{BaTiO}_{3} / \mathrm{SrTiO}_{3}$ (BTO/STO) has been reported [40], in which the ferroelectricity of BTO can introduce a polar discontinuity at the interface similar to the LAO with alternating polar planes. And we can predict that the interaction between the interfaces of LAO/STO and BTO/STO may result in novel phenomena. 
In this Chapter, we performed first-principle calculations systematically to tune the 2DEG at the n-type LAO/STO interface through a ferroelectric substrate BTO and investigated the ferroelectric field effect on the carrier density of the 2DEG. Our calculations show that the carrier density at the n-type LAO/STO interface is greatly changed as the polarization of BTO reverses. The electronic reconstruction at the interface resulted from the change of the intrinsic electric field in LAO due to the ferroelectric field effect. This suggests new possibilities to tune the 2DEG at the LAO/STO interface and new paths to develop high performance transistors.

\subsection{Computational Method}

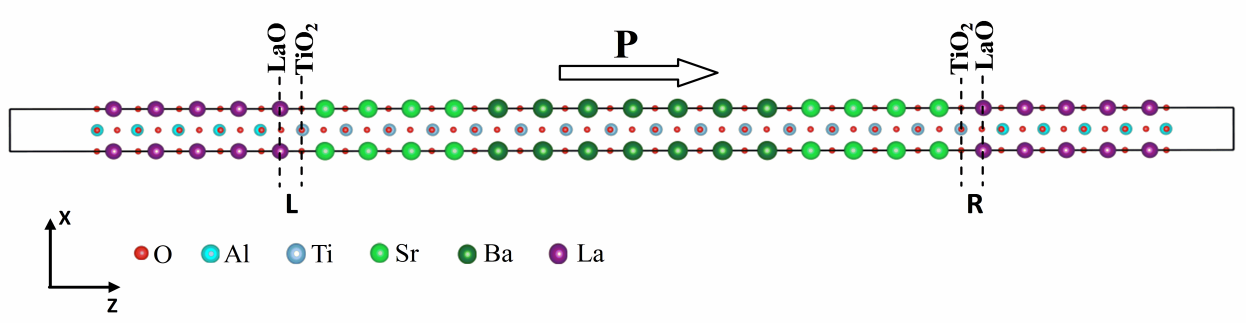

Figure 2.1: (color online). Schematic of the LAO/STO/BTO superstructure with two symmetrical n-type LAO/STO interfaces. The direction of polarization in BTO is from left to right along the (001).

The calculations are performed within density functional theory (DFT) using the generalized gradient approximation of Perdew, Burke, and Ernzerfof (PBE), as implemented in the Vienna ab initio simulation package (VASP). The GGA+U method is adopted with an on-site Coulomb repulsion of $\mathrm{U}=5.8 \mathrm{eV}$ and an exchange interaction of $\mathrm{J}=1 \mathrm{eV}$ for Ti-3d orbital and $\mathrm{U}=9 \mathrm{eV}$ and $\mathrm{J}=1 \mathrm{eV}$ for La- $4 \mathrm{f}$ orbital. The energy cutoff for the plane wave basis set is chosen to be $500 \mathrm{eV}$. A $8 \times 8 \times$ 1 Monkhorst-Pack k-point meshes is used for the LAO/STO/BTO superstructure Brillouin-zone integration and $8 \times 8 \times 8$ for the bulk LAO, BTO and STO calculations. The structures are relaxed until the residual force become less than $30 \mathrm{meV} / \AA$. The energy is converged to $10^{-5} \mathrm{eV} /$ cell.

For convenience, we adopt a symmetrical structure similar to that used in the investigation of magnetoelectric effect [41], which allows us to study the effect of polarization reversal at the interface of a single heterostructure by comparing properties of the two interfaces. The slab is constructed of 10 u.c. LAO, 8 u.c. STO, and 7.5 u.c. BTO with two symmetrical n-type LAO/STO interfaces along the (001) direction. 7.5 u.c. BTO is sufficient to sustain the ferroelectricity [42]. To avoid spurious electric field, we construct a vacuum of $15 \AA$ to separate the slab as shown in Fig. 2.1. Test calculations show that the vacuum layer is sufficiently thick. The 
schematic for the LAO/STO/BTO superstructure with polarization in BTO pointed to the right is shown in Fig. 2.1.

Bulk LAO is orthorhombic (R-3c) at room temperature and shows a pseudocubic structure as LAO is epitaxially grown on the BTO substrate. We optimize the cubic lattice constant of STO and LAO to be $3.949 \AA$ and $3.81 \AA$, respectively, which agrees well with the experimental value of $3.905 \AA$ and $3.789 \AA$ [43]. The overestimation of equilibrium lattice parameters is well known for the GGA exchange-correlation potential used in the calculations. Considering the tetragonal phase of BTO at room temperature, the calculated lattice constant $\mathrm{a}=3.994$ $\AA$ and $\mathrm{c} / \mathrm{a}=1.015$ compare favorably with the experimental value of $\mathrm{a}=3.998 \AA$ and $c / a=1.011$ [44], respectively. To simulate the epitaxial growth on BTO substrate, the in-plane lattice constant is fixed to the GGA equilibrium lattice constant of tetragonal BTO $(\mathrm{a}=\mathrm{b}=3.994 \AA)$. Under this constraint, the out-of-plane lattice constant of bulk LAO and STO are obtained by minimizing the total energy giving the c/a ratios of 0.87 and 0.92 , respectively. The energy gaps for bulk STO, LAO and BTO are $2.0 \mathrm{eV}\left(\mathrm{E}_{g}^{\text {expt }}=3.2 \mathrm{eV}[13]\right), 3.6 \mathrm{eV}\left(\mathrm{E}_{g}^{\text {expt }}=5.6 \mathrm{eV}[13]\right)$ and $2.0 \mathrm{eV}$ $\left(\mathrm{E}_{g}^{\text {expt }}=3.0 \mathrm{eV}[45]\right.$ ), respectively, where the underestimation of energy gap is due to the local nature of the functional in DFT and would not change our conclusions.

\subsection{Results and Discussion}

To investigate the effect of polarization of BTO on the electronic characteristics of the n-type LAO/STO interface, we first consider the supercell with paraelectric BTO, which is stable as the supercell is fully relaxed. The density of states (DOS) projected on unit-cell bilayers for left and right LAO/STO interfaces are plotted in Fig. 2.2(a) and Fig. 2.2(b) (red line), respectively. We find that the 2DEG is constrained at the LAO/STO interfaces and does not extends into deeper STO layers as previous reports $[22 ; 46]$. This can be attributed to the tensile strain $(2.4 \%)$ on STO [47]. As reported by previous theoretical works [48], the valence band maximum of each unit-cell bilayer in LAO shifts linearly upward away from the interface due to the intrinsic electric field until it reaches the conduction band bottom of STO near the interface. As a result, the electrons from the valence band of surface $\mathrm{AlO}_{2}$ layer are transferred to the conduction band of Ti $3 \mathrm{~d}$ orbitals near the interface to compensate the polar discontinuity at the interface. The charge transfer can be estimated by integrating the density of states projected on Ti 3d orbitals from the conduction band minimum up to the Fermi energy at the interface. The evaluated carrier density in STO is about $0.09 \mathrm{e} /$ cell, greatly lower than the theoretical 0.5 e/cell for the existence of polar distortions in LAO and STO [48].

The relative displacements of $\mathrm{Ti}$ atoms with respect to $\mathrm{O}$ atoms are found to be about $0.10 \AA$ in bulk BTO, corresponding to a ferroelectric polarization of about $0.22 \mathrm{C} / \mathrm{m}^{2}$ along the 001 direction calculated using the Berry phase method, which 

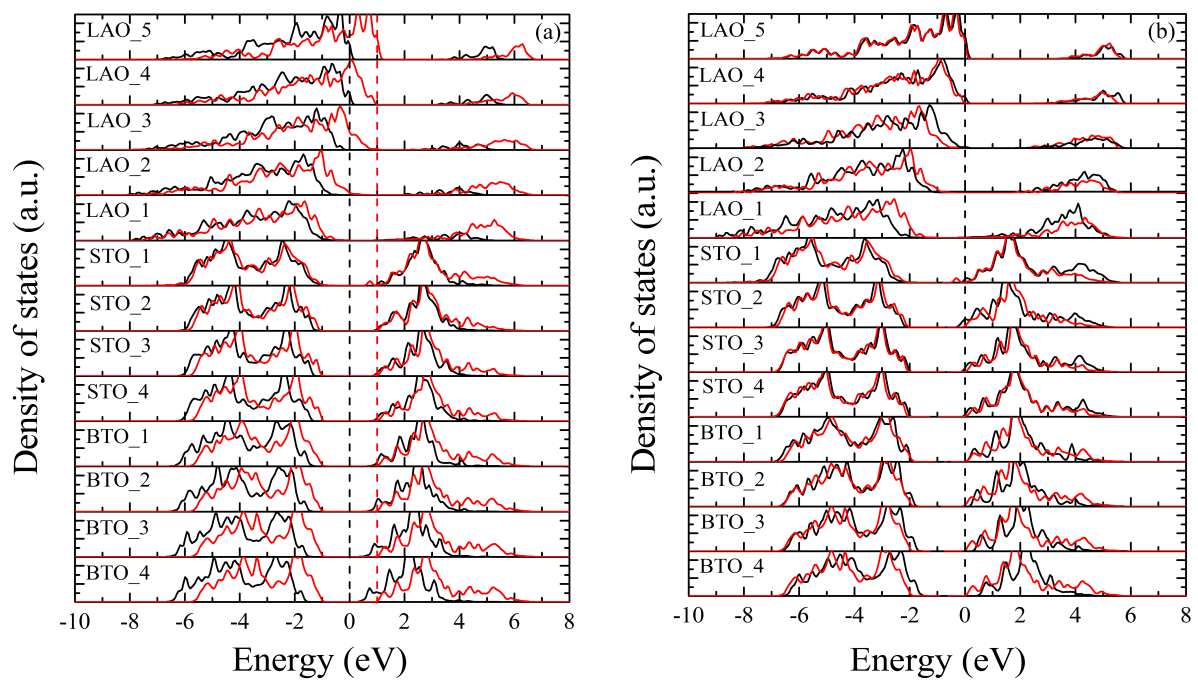

Figure 2.2: (color online). The density of states projected on the subunit-cell with unpolarized (red line) and polarized (black line) BTO for the left (a) and right (b) n-type LAO/STO interfaces. The vertical black dashed lines indicate the Fermi level located at $0 \mathrm{eV}$. In (a), the DOS for the unpolarized condition was shifted by $1 \mathrm{eV}$ to align with the conduction band of the system with the polarized BTO. Its Fermi level is marked with a red dashed line. The numbers in figures indicate the LAO or STO unit cell layers at various distances away from the interface.

consists well with the experimental and previous theoretical calculations [49; 50; 51].

We polarize the BTO from left to right along (001) as shown in Fig. 2.1. From Fig. 2.2(a), it can be seen that the DOS of left n-type LAO/STO interface (black line) with polarization pointed away from the interface exhibits insulator character as the conduction band bottom of STO lies above the Fermi level. It is clear that a gap of about $1 \mathrm{eV}$ occurs between the top valance band of surface LAO and the bottom conduction band of STO layer at the interface. On the contrary, from Fig. 2.2(b) (black line), the right n-type LAO/STO interface with polarization pointed to the interface is conducting with a large distribution of electronic states on the Fermi level. In addition, the 2DEG spread over four STO layers as shown in Fig. 2.2(b). The DOS of BTO shows the same trend to LAO in Fig. 2.2(a) that decreases the polar drop in LAO, while it shows the opposite trend in Fig. 2.2(b) that enhances the polar drop in LAO. Therefore, the n-type LAO/STO interface goes through a metal-to-insulator transition as the polarization of BTO reverses. In Fig. 2.2(a) (black line), the DOS of BTO shows the same trend as that in LAO, indicating that the build-in electric field in BTO and LAO have the same directions. In contrast, the 
BTO exhibits an opposite electric field direction to the LAO in Fig. 2.2(b). It should be mentioned that there is considerable density of electronic states on the Fermi level at the left surface $\mathrm{AlO}_{2}^{-1}$ layer. For the sake of the low-lying STO conduction band at the right LAO/STO interface, the electric field in left LAO and BTO can be compensated by transferring the electrons at the left surface $\mathrm{AlO}_{2}^{-1}$ layer to the $\mathrm{TiO}_{2}^{0}$ layer near the right $\mathrm{LAO} / \mathrm{STO}$ interface shown in Fig. 2.2(a), (b). However, this would not change the insulating characteristic at the left LAO/STO interface.

(a)

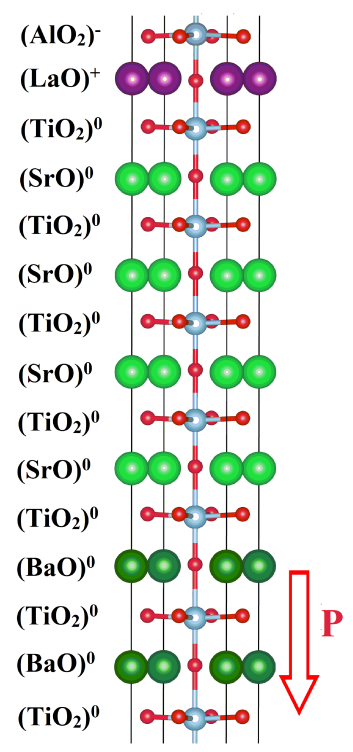

(b)

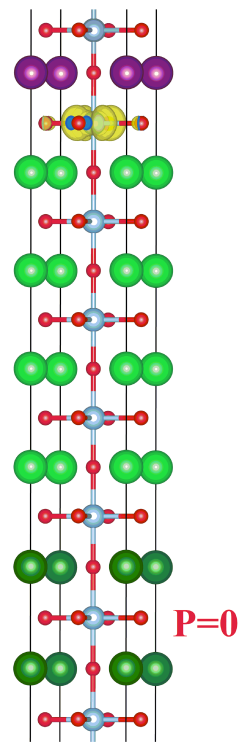

(c)

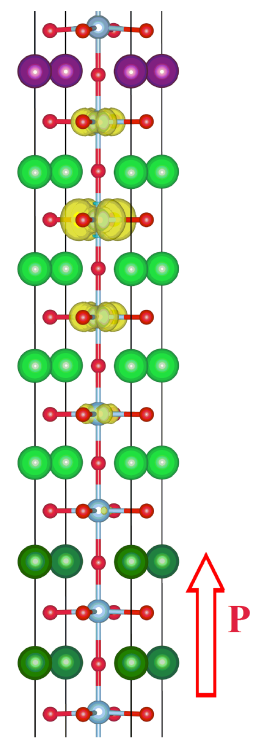

Figure 2.3: (color online). Charge density projected on the bands forming the 2DEG in LAO/STO/BTO system for (a) polarization in BTO pointed away from the interface, (b) unpolarized BTO, and (c) polarization in BTO pointed to the interface. The arrows indicate the direction of the polarization in BTO. The same isovalue of 0.0038 is used to produce the charge density plots.

To have a direct view of the spatial distribution of the 2DEG, the charge density projected on the bands forming 2DEG in LAO/STO/BTO system with various polarizations has been plotted in Fig. 2.3. The 2DEG is constrained at the interface under $\mathrm{P}=0$. The carrier density of $2 \mathrm{DEG}$ is enhanced as $\mathrm{P}$ points to the interface, while it decreases to zero as $\mathrm{P}$ reverses. These results consist well with the DOS shown in Fig. 2.2. In our calculations, we adopt BTO as the substrate which induces a tensile strain on STO and makes the Ti $d_{x y}$ orbitals of STO lie on the lowest energy level [30]. The 2DEG comprises mostly $d_{x y}$ orbitals as shown in Fig. 2.3(b), (c). It is intriguing that the 2DEG mainly lies on STO_2 and decreases at the deeper STO layers, i.e., STO_3 and STO_4 layers. This can be attributed to the electrostatic 
attraction by the bound charges induced by the polarization in BTO.

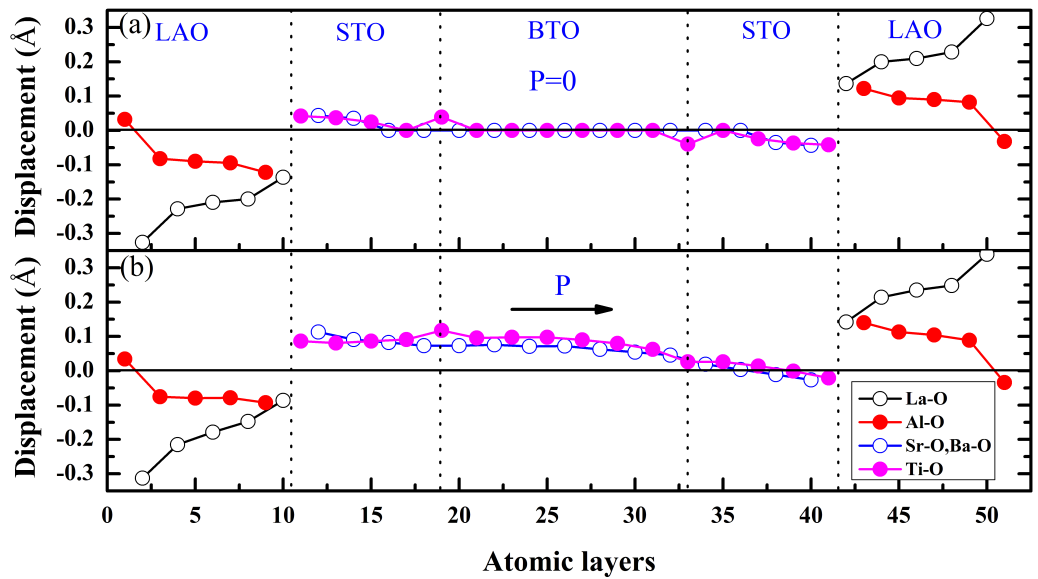

Figure 2.4: (color online). The cations ( $\mathrm{La}, \mathrm{Sr}, \mathrm{Ba}, \mathrm{Al}, \mathrm{Ti}$ ) displacements relative to the oxygen anion in each layer are displayed for the system with (a) unpolarized BTO and (b) polarized BTO.

Fig. 2.4(a), (b) shows the relative displacements of cations relative to the oxygen anions at each layer with un-polarized and polarized BTO, respectively. The polar displacements in STO and LAO are supposed to be the origin of the critical thickness in the formation of 2DEG [48]. The polar displacement in LAO and STO with cations moving away from the interface relatively to anions can compensate the intrinsic electric field in LAO. From Fig. 2.4(a), we can see that the polar displacement in LAO and STO consistent well with experimental results and previous calculations. It is intriguing that the relative displacements of $\mathrm{Al}$ and $\mathrm{O}$ atoms in LAO surface layer is opposite to that in other LAO layers. In contrast, as BTO is polarized, it is clear that the ionic displacements of both sides are divergent as shown in Fig. 2.4(b). The relative ionic displacement of Ti with respect to $\mathrm{O}$ atoms in the middle layer of BTO is about $0.097 \AA$, a little bit less than the bulk value. The most obvious change of the ionic displacements due to the polarization of BTO are the rumpling in STO. Compared with un-polarized situation, the relative displacements of Ti and O atoms in left STO are enhanced to an average value of $0.08 \AA$, while it is suppressed in right STO. The STO epitaxially grown on BTO substrate suffers a tensile strain, which can be easily polarized [36]. Due to the "dead layer" near the right interfacial $\mathrm{LaO}^{+}$layer, the polarization in right STO is negligible. In addition, the suppressed polar distortion in right STO contributes to the enhancement of the charge density of 2DEG.

To quantify the effect of polarization of BTO on the electronic structure of the n-type LAO/STO interface and the ionic displacements, the planer-averaged elec- 

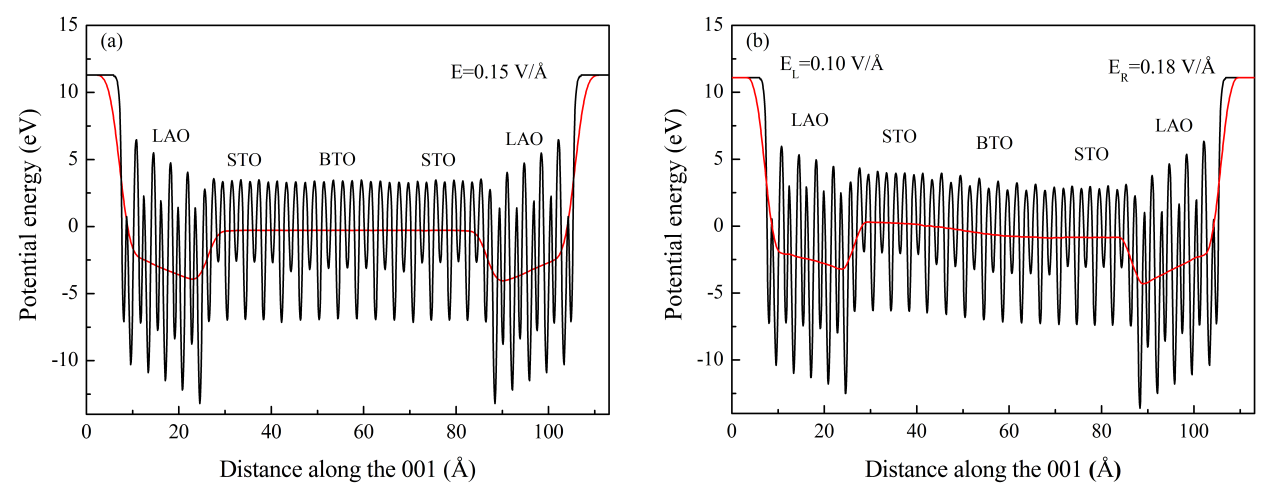

Figure 2.5: (color online). The planar-averaged (dark) and the macroscopically averaged (red) potential energy of LAO/STO/BTO superstructure for the (a) unpolarized and (b) polarized condition as a function of distance along the 001.

trostatic potential [52] in the LAO/STO/BTO superstructure is plotted along $\mathrm{z}$ axis for unpolarized and polarized supercells, respectively in Fig. 2.5(a) and (b). From the slope of the macroscopic-averaged electrostatic potential, the electric field for left and right LAO in polarized situation is estimated to be $0.10 \mathrm{~V} / \AA$ and $0.18 \mathrm{~V} / \AA$, respectively, while the electric field is estimated to be $0.15 \mathrm{~V} / \AA$ for the unpolarized situation. When BTO is polarized from left to right, it is evident that the intrinsic electric field in left LAO is suppressed, while the right is enhanced. The difference of electric field between two sides can be attributed to the ferroelectric field effect of BTO.

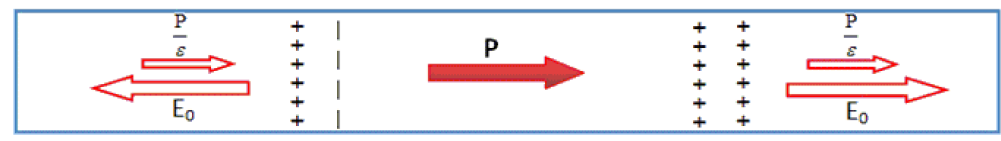

Figure 2.6: (color online). The schematic of the ferroelectric field effect on the intrinsic-field of LAO as described in text.

To make it clear, the schematic of the polarization effect on the intrinsic electric field of LAO is shown in Fig. 2.6. The polarization of BTO produces positive (negative) bound charges at right BTO/STO (left LAO/STO) interface [indicated in Fig. 2.6]. The bound charges create an additional electric field in LAO, which is parallel and anti-parallel to the intrinsic electric field $\mathrm{E}_{0}$ in right and left LAO, respectively. The electric field in right LAO is enhanced, leading to an increase of the charge density at the interface. On the other hand, the left LAO/STO interface becomes insulating due to the decrease of the intrinsic electric field in left LAO. 
As a result, a metal-to-insulator (MIT) transition is observed at the LAO/STO interface with the polarization reversal. The enhanced (suppressed) electric field in right (left) LAO leads to a small increase (decrease) of the polar distortion in LAO to balance the imposed electric field as shown in Fig. 2.4(b). For thick BTO, The conductance at the LAO/STO can be tuned sensitively from metal to insulator with the direction and magnitude of the polarization of BTO.

The intrinsic electric field E of LAO in presence of BTO polarization can be described as follows:

$$
E=E_{0} \pm \Delta E \mp \frac{P}{\epsilon_{L A O}}
$$

where $\mathrm{E}_{0}$ represents the intrinsic value of electric field in LAO, $\mathrm{P}$ represents the average polarization of BTO and STO, $\triangle E$ represents the compensating electric field induced by the change of polar displacement in LAO $\left(\Delta E=\sum Z_{i} \Delta z_{i} / V_{L A O} \epsilon_{L A O}\right)$, $\mathrm{V}_{L A O}$ is the volume of LAO. Using the calculated value of $\Delta E(0.055 \mathrm{~V} / \AA)$ and the averaged polarization $\mathrm{P}=0.15 \mathrm{C} / \mathrm{m}^{2}$ (calculated using Berry Phase method), we estimate the dielectric constant of LAO to be $\epsilon_{L A O}=18 \epsilon_{0}$, which is less than the bulk value of $24 \epsilon_{0}$. This can be attributed to the thin thickness of LAO.

Below the critical thickness, the polar potential in LAO is not enough to overcome the gap of STO. The polar potential will be sustained in LAO and increase with the thickness of LAO, leading to a decrease of the gap between surface $\mathrm{O} 2 \mathrm{p}$ valence band states and interface Ti $3 \mathrm{~d}$ conduction band states [48; 53]. Above the critical thickness, the polar potential in LAO exceeds the gap of STO and the part over the gap $\left[\Delta V_{L A O}=E t_{L A O}-e_{g}-\left(e_{V B M}^{S T O}-e_{V B M}^{L A O}\right)\right]$ will be compensated by the electronic reconstruction, leading to a conducting interface, while a potential drop equal to the gap of STO will still be sustained in LAO. The theoretical charge density of 2DEG $0.5 \mathrm{e} /$ cell is the limiting case as the thickness of LAO increases.

For the system with unpolarized BTO, the intrinsic electric field in LAO is estimated from Fig. 2.5(a) to be $0.15 \mathrm{~V} / \AA$, with an electric potential $V=e \times(3.7 \AA) \times$ $(5 \mathrm{cells}) \times 0.15 \mathrm{~V} / \AA=2.78 \mathrm{eV}$, larger than the calculated band gap of STO $2.0 \mathrm{eV}$ (calculated value of the valence-band offset is about $0.1 \mathrm{eV}$ [54] ). The compensating electronic dipole is $D=-0.09 e \times(3.7 \AA) \times 5$ cells $=-1.67 e \AA$. Using the calculated dielectric constant of LAO, we can get the compensating electronic potential $\Delta V=4 \pi e \frac{D}{\epsilon(5 \times 3.7 \AA)^{2}}=0.61 \mathrm{eV}$, fitting well with the $\Delta V$ calculated by $(2.78 \mathrm{eV})-(2.0 \mathrm{eV})-0.1 \mathrm{eV}=0.68 \mathrm{eV}$.

As to the polarized condition, the $\Delta V$ in LAO is estimated to be $e \times 3.7 \AA \times$ $(5 \mathrm{cells}) \times(0.1 \mathrm{~V} / \AA)-2.1 \mathrm{eV}=-0.25 \mathrm{eV}$ and $e \times 3.7 \AA \times(5 \mathrm{cells}) \times(0.18 \mathrm{~V} / \AA)-2.1 \mathrm{eV}=$ $1.23 \mathrm{eV}$ for left and right side, respectively. Thus, the polar potential in left LAO does not reach the gap of STO, leading to an insulator characteristic at the left LAO/STO interface with the conduction band bottom of STO lying $1 \mathrm{eV}$ above the Fermi level, while the right LAO has a large polar potential with an enhanced charge density of $2 \mathrm{DEG}$ at right LAO/STO interface. It should be noted, due to the residul polar 
potential of $1 \mathrm{eV}$ in $\mathrm{BTO}$, the electrons from left surface $\mathrm{AlO}_{2}^{-1}$ layer are transferred to the right LAO/STO interface with $\Delta V_{L A O}=e \times 3.7 \AA \times(5 c e l l s) \times(0.1 \mathrm{~V} / \AA)+$ $(1 \mathrm{eV})-2.1 \mathrm{eV}=0.75 \mathrm{eV}$. A total charge density of $0.24 \mathrm{e} /$ cell appears in right STO, estimated by integrating the density of states projected on Ti 3d orbital from the conduction band minimum up to the Fermi energy and from the bottom of the STO slab to the interface. Due to the conservation of charge in the system, the 2DEG in the supercell must originate from both left and right $\mathrm{AlO}_{2}$ surface layers, which are $0.10 \mathrm{e} / \mathrm{cell}$ and $0.14 \mathrm{e} / \mathrm{cell}$, respectively, estimated by integrating the density of states projected on $\mathrm{O} 2 \mathrm{p}$ orbital from the valence band maximum down to the Fermi energy and from the top of the LAO slab to the interface. The electric reconstruction near right LAO/STO interface compensates both side polar potentials, including the polarization in BTO. It is noted that the two-dimensional gas formed at right interface screen the polarization in BTO, leading to a substantially reduced potential drop of about $0.016 \mathrm{eV} / \AA$ across the ferroelectric film.

In short, the ferroelectric field in BTO tunes the 2DEG at the LAO/STO (001) interface through altering the intrinsic electric-field in LAO, which provides a new way to tune the 2DEG at the LAO/STO interface. The change of intrinsic electric field in LAO is also responsible to the electric-field effect on 2DEG which changes the polar order in STO, especially at low temperature due to the phase transition in STO. Ferroelectricity was induced in $\mathrm{SrTiO}_{3}$ by the isotope exchange of ${ }^{18} \mathrm{O}$ for ${ }^{16} \mathrm{O}$ without the application of external fields, which can enhance the ferroelectric field effect on 2DEG discussed above [55]. A similar temperature-dependent metal-toinsulator transition in the LAO/BTO interface epitaxially grown on the STO substrate has been experimentally reported by Chae etc [56], which is attributed to the enhanced electronic carrier concentration due to the ferroelectric transition of BTO thin film. Based on the "polar catastrophe" model, the polar discontinuity at the interface provides the driving force for the formation of 2DEG. By calculations, it is reasonable to conclude that the intrinsic electric field in LAO plays the dominant role in the electronic reconstruction, which determines the potential drop in $\mathrm{LAO}$ and therefore the energy gap between $\mathrm{O} 2 \mathrm{p}$ orbital in the surface $\mathrm{AlO}_{2}$ layer and $\mathrm{Ti} 3 \mathrm{~d}$ orbital of the interface $\mathrm{TiO}_{2}$ layer due to the alignment of valence band of LAO and STO at the interface.

\subsection{Conclusion}

In summary, we have performed first principle calculations to explore the effect of the polarization of BTO on the electronic properties at the n-type LAO/STO interface. A MIT occurs at the LAO/STO (001) interface as the polarization of BTO reverses. The analysis on the LAO/STO/BTO superstructure shows that the polarization of BTO significantly changes the intrisinc electric field in LAO and leads to the electronic reconstrction at the interface, which provides a mechanism to make 
us understand better about the modulation of 2DEG and guide us design new novel device. In practice, the charge density at the n-type interface can be tuned sensitively through the polarization of ferroeltctric substrate, which has great potential in the application of high-frequency transitors, nonvolatile memory devices, and other novel electric devices in the future. 


\section{3 \\ High temperature itinerant ferromagnetism in $p$-doped monolayers of $\mathrm{MoS}_{2}$}

We use density functional theory to explore the possibility of making the semiconducting transition-metal dichalcogenide $\mathrm{MoS}_{2}$ ferromagnetic by introducing holes into the narrow Mo $d$ band that forms the top of the valence band. In the single impurity limit, the repulsive Coulomb potential of an acceptor atom and intervalley scattering lead to a twofold orbitally degenerate effective-mass like $e^{\prime}$ state being formed from Mo $d_{x^{2}-y^{2}}$ and $d_{x y}$ states, bound to the $K$ and $K^{\prime}$ valence band maxima. It also leads to a singly degenerate $a_{1}^{\prime}$ state with $M o d_{3 z^{2}-r^{2}}$ character bound to the slightly lower lying valence band maximum at $\Gamma$. Within the accuracy of our calculations, these $e^{\prime}$ and $a_{1}^{\prime}$ states are degenerate for $\mathrm{MoS}_{2}$ and accommodate the hole that polarizes fully in the local spin density approximation in the impurity limit. With spin-orbit coupling included, we find a single ion magnetic anisotropy of $\sim 5 \mathrm{meV}$ favouring out-of-plane orientation of the magnetic moment. Pairs of such hole states introduced by $\mathrm{V}, \mathrm{Nb}$ or Ta doping are found to couple ferromagnetically unless the dopant atoms are too close in which case the magnetic moments are quenched by the formation of spin singlets. Monte Carlo calculations allows us to estimate ordering temperatures as a function of $x$. For $x \sim 9 \%$, Curie temperatures as high as $100 \mathrm{~K}$ for $\mathrm{Nb}$ and Ta and in excess of $160 \mathrm{~K}$ for $V$ doping are predicted. Factors limiting the ordering temperature are identified and suggestions made to circumvent these limitations.

"published as: [Y.Q. Gao, N. Ganguli, and P. J. Kelly. Phys.Rev.B 99,220406(R),2019];[Y.Q. Gao, N. Ganguli, and P. J. Kelly. Phys. Rev. B 100, 235440, 2019] 


\subsection{Introduction}

The discovery of ferromagnetism in (In,Mn)As [57] and (Ga,Mn)As [58] and predictions for achieving room temperature ordering [59] sparked a huge effort to realize a dilute magnetic semiconductor (DMS) that might lead to a semiconductorbased spin electronics ("Spintronics"). After twenty-five years of intensive research, the maximum ordering temperature has stagnated at values too low for extensive applications [60]. The number of material systems being considered has proliferated but it is not clear what the fundamental limit is to the ordering temperature achievable in any particular material system. There are many reasons for the low ordering temperatures $[61 ; 62]$ but the essential dilemma is that the open $d$ shell states of magnetic impurities like Mn are quite localized. While this favours the onsite exchange interaction that is the origin of the Hund's-rule spin alignment and makes the ionic moment insensitive to temperature, it leads to weaker exchange interactions between pairs of impurity ions that determine the Curie temperature $T_{C}$, the ferromagnetic ordering temperature. To increase $T_{C}$, the concentration of impurity atoms has to be increased. This is accompanied by a variety of adverse effects such as a nonuniform distribution of magnetic impurities or the formation of antisite defects that are electron donors which counter the intended increase in the concentration of holes. In many semiconductors, transition metal ions introduce "deep levels", tightly bound partially occupied states in the fundamental gap of the semiconductor. At high dopant concentrations, these form deep impurity bands that dominate the (transport) properties of a material that is no longer a semiconductor and from the electronic structure point of view, is an entirely new material.

In a quite different context, it was long believed that long-range magnetic ordering would not be possible in two-dimensional (2D) materials [63; 64]. However the observation of ferromagnetism in ultrathin epitaxial layers of e.g., Fe on Au substrates demonstrated that the Mermin-Wagner theorem is not watertight, violation of the proof usually being attributed to magnetocrystalline anisotropy [65]. The recent observation of ferromagnetic ordering in two different chromium-based 2D crystalline materials $\mathrm{Cr}_{2} \mathrm{Ge}_{2} \mathrm{Te}_{6}$ [66] and $\mathrm{CrI}_{3}$ [67] nonetheless attracted considerable attention [68]. One reason was because of the general interest in 2D materials, triggered by spectacular observations on graphene $[1 ; 69 ; 70]$. This interest was reinforced by the realization that the properties of semiconductors like $\mathrm{MoS}_{2}$ could also be importantly different in few- and mono-layer form $[71 ; 11 ; 72]$ and was compounded by the desirability of stacking layers of 2D materials with different properties [73] whereby the lack of a ferromagnetic material in a vast profusion of 2D materials was a striking lacuna [74]. Because the Curie temperatures of monolayers of the chromium based materials $[66$; 67] is low, $\lesssim 50 \mathrm{~K}$, the very recent reports that the transition metal dichalcogenide $\mathrm{VSe}_{2}$ [75] and $\mathrm{Fe}_{3} \mathrm{GeTe}_{2}$ [76] exhibit ferromagnetism at room temperature acquires huge significance.

The ferromagnetism of $\mathrm{VS}_{2}$ and $\mathrm{VSe}_{2}$ was predicted with the aid of density 
functional theory (DFT) calculations [77]. The driving force behind the magnetic ordering can be understood in terms of the band structure of the nonmagnetic $1 \mathrm{H}$ phase shown in Fig. 3.1(a) that is very similar to that of the isostructural $\mathrm{MoS}_{2}$ shown in Fig. 3.1(b) but with one valence electron per formula unit less so that it is metallic with the Fermi level situated in the middle of the solid red band. Bulk multilayered $\mathrm{MoS}_{2}$ is a non-magnetic semiconductor with an indirect bandgap of about $1 \mathrm{eV}$. In monolayer form it was predicted to have a larger, direct gap [78] and this was confirmed experimentally where direct gaps of $\sim 1.8 \mathrm{eV}$ have been reported $[71 ; 11]$. In the figure, the "nominal" Mo $4 d$ bands are indicated in red, the black bands are sulphur-derived $3 p$ bands. The interaction of the Mo- $d$ and S- $p$ states is such that a large covalent bonding-antibonding gap is formed leaving a single Mo- $d$ band (solid red line) with mixed $\left\{d_{x^{2}-y^{2}}, d_{x y}, d_{3 z^{2}-r^{2}}\right\}$ character in the fundamental band gap [79; 80]. For $\mathrm{MoX}_{2}$, this band is completely filled but for $\mathrm{VX}_{2}$ it is only half full. The dispersion of only about $1 \mathrm{eV}$ leads to a high average density of states of $\sim 2$ states/eV and the gain in energy achieved by exchange-splitting this narrow band more than offsets the kinetic energy cost. The bandwidth reduction in 2D that leads to larger band gaps is favourable for itinerant ferromagnetism because of the higher average densities of states (DoS) than in three dimensions. Likewise $3 d$ elements are more favourable than $4 d$ and $4 d$ more favourable than $5 d$ because of the greater localization of the $d$ electrons and concomittant smaller bandwidth as the principal quantum number decreases.

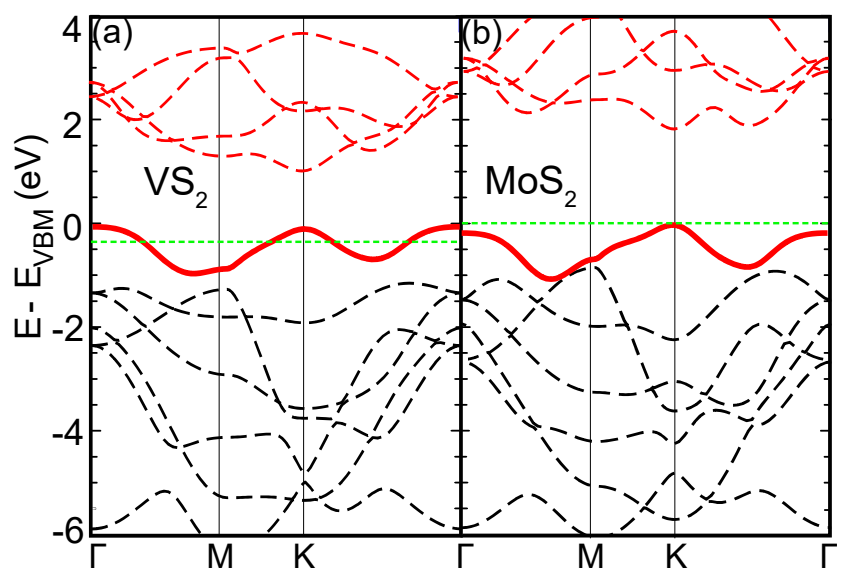

Figure 3.1: Non spin-polarized band structures of monolayers of trigonal prismatic $1 \mathrm{H} \mathrm{VS}_{2}$ (a) and $\mathrm{MoS}_{2}$ (b). The Fermi level is indicated by a horizontal dashed green line. The valence band maximum (VBM) at the $\mathrm{K}$ and $\mathrm{K}^{\prime}$ points has mixed $d_{x^{2}-y^{2}}$ and $d_{x y}$ character. The slightly lower-lying valence band maximum at the $\Gamma$ point has $d_{3 z^{2}-r^{2}}$ character.

A number of intrinsic defects have been found to form local moments [81; 82; 
83; 84; 85] in $\mathrm{MX}_{2}$ materials and suggestions have been made to make the $\mathrm{MX}_{2}$ materials magnetic by adsorption of impurity atoms $[86 ; 81 ; 87 ; 82]$, or by substituting $\mathrm{M}$ or $\mathrm{X}$ atoms with impurity atoms $[88 ; 89 ; 90 ; 91 ; 92 ; 87 ; 93 ; 94 ; 95 ; 96$; $82 ; 97 ; 98 ; 99 ; 100 ; 101 ; 102 ; 103 ; 104 ; 105]$. Even though the Mermin-Wagner theorem $[63 ; 64]$ tells us that there is no long range ordering in two dimensions for isotropic Heisenberg exchange, few attempts have been made to determine the exchange coupling between magnetic impurities $[91 ; 92 ; 87 ; 94 ; 95 ; 101 ; 105]$ and it was only very recently that the magnetic anistropy of a defect was calculated, for an antisite defect in $\mathrm{MoS}_{2}$ [85]. Replacing some of the M atoms with Hund'srule coupled transition metal atoms like $\mathrm{Mn}$ or Fe gives rise to deep impurity levels in the semiconductor gap. Where attempts have been made to estimate the Curie temperature, the predicted values are either very low or the concentration of transition metal dopant is so high that the doped material is no longer a semiconductor [89; 91; 92; 94; 95; 104]. Based upon the electronic structure shown in Fig. 3.1(b), we explore a different approach to making $\mathrm{MoS}_{2}$ ferromagnetic in this manuscript [106].

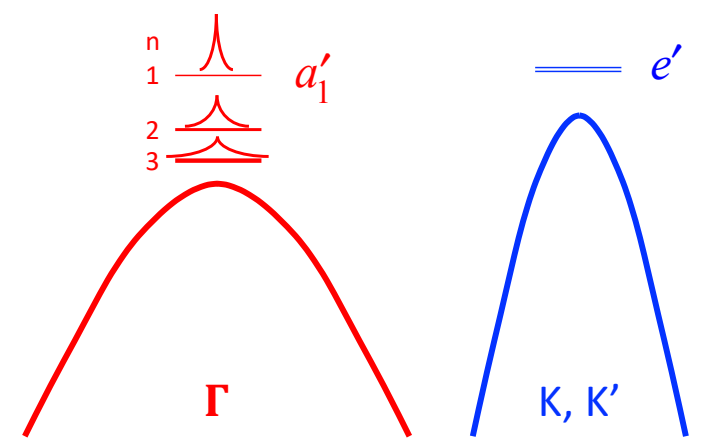

Figure 3.2: Schematic of the effective mass acceptor states bound to the valence band maxima (VBM): an $e^{\prime}$ state bound to the $\mathrm{K}-\mathrm{K}^{\prime} \mathrm{VBM}$ and an $a_{1}^{\prime}$ state to the $\Gamma$ VBM. $n$ is the principal quantum number and only $n=1,2,3$ levels of the Rydberg series are sketched at the $\Gamma$ point.

Group VIB Mo has a $4 d^{5} 5 s^{1}$ electronic configuration and, in a dichalcogenide like $\mathrm{MoS}_{2}$, is nominally $\mathrm{Mo}^{4+}$ with one up-spin and one down-spin $d$ electron so it is nonmagnetic as seen in Fig. 3.1(b). When a Mo atom is substituted by a group $\mathrm{VB}$ atom like $\mathrm{V}, \mathrm{Nb}$ or $\mathrm{Ta}$, then the dopant atom e.g. $\mathrm{V}^{4+}$, has a single unpaired $d$ electron and a single hole is thereby introduced into the narrow Mo $4 d$ band; substitution of a group IVB atom (Ti, Zr, Hf) will introduce two holes per dopant atom. In the impurity limit, the asymptotic Coulomb potential leads to a series of hydrogenic states bound to the top of the valence band; to the maxima at the $\mathrm{K}$ and $\mathrm{K}^{\prime}$ points with mixed Mo $d_{x^{2}-y^{2}}$ and $d_{x y}$ character and to the slightly lower valence band maximum at the $\Gamma$ point with Mo $d_{3 z^{2}-r^{2}}$ character and a large effective mass 
[107].

The aim of this Chapter is to determine if there are dopant atoms whose potential is sufficiently similar to that of the host Mo atom that only weakly bound, effective-mass like states are formed above the valence band edge, Fig. 3.2. At low concentrations these bound states should polarize and form impurity bands that have such a high density of states that they remain exchange split [108]. At finite temperatures these polarized bound holes will be excited into the valence band giving rise to a DMS. The key objectives of this Chapter are to determine (i) whether single acceptor dopant atoms give rise to polarized effective-mass like states in the $\mathrm{MoS}_{2}$ host system and to determine the position of these states with respect to the valence band edge; (ii) whether the interaction between pairs of dopant atoms favours ferromagnetic or antiferromagnetic alignment and to identify the nature of the interaction, Zener $p$ - $d$ type, double exchange etc. $[61 ; 62]$ and understand the factors determining it; (iii) the magnetic anisotropy of single impurities, the so-called single ion anisotropy (SIA); (iv) the ordering temperature and express it in terms of parameterized models that describe the dopant-induced states and their interactions in order to identify the most promising regions of parameter space to realize a room temperature DMS.

To do this we use density functional theory total energy calculations to determine ground state energies of single acceptor impurities. We outline the methods used and give some technical details specific to the present work in Sec. 3.2. Our results are presented in Sec. 4.3 beginning with a study of the single impurity limit of a substitutional vanadium atom in Sec. 3.3.1 including the effects of spin polarization and local atomic relaxation. The binding of pairs of $\mathrm{V}$ dopants is considered in Sec. 3.3.2 and their magnetic "exchange" interaction in Sec. 3.3.3 with special attention being devoted to understanding the quenching of the magnetic moments of close pairs of impurity ions. In Sec. 3.3.4 we briefly compare V with $\mathrm{Nb}$ and Ta. Sec. 3.4 is concerned with the question of magnetic ordering and begins with a study of the single ion anisotropy of V impurities in Sec. 3.4.1 to justify using an Ising spin model with the exchange interactions from Sec. 3.3.3 and the Monte Carlo techniques described in Sec. 3.4.2 to estimate ordering temperatures in Sec. 3.4.3. A comparison of our findings with other calculations in Sec. 3.5 leads us to consider how using the generalized gradient approximation (GGA) would alter our local density approximation (LDA) results. After a brief discussion in Sec. 3.6 some conclusions are drawn in Sec. 3.7.

\subsection{Computational Details}

Calculations of the total energy and structural optimizations were carried out within the framework of density functional theory (DFT) using the projector augmented wave (PAW) method [109] and a plane-wave basis set with a cut-off energy of 400 $\mathrm{eV}$ as implemented in the vasP code $[110 ; 111 ; 112]$. Monolayers of $\mathrm{MX}_{2}$ period- 
ically repeated in the $c$ direction were separated by more than $20 \AA$ of vacuum to avoid spurious interaction.

Table 3.1: In-plane lattice constant $a$, distance between sulphur atoms $d_{\mathrm{SS}}$ (thickness of an $\mathrm{MoS}_{2}$ monolayer), Mo-S bond length $d_{\mathrm{MoS}}$, energy gap $\Delta \varepsilon_{g}$, and energy difference between the valence band maxima (VBM) at the $\mathrm{K}$ and $\Gamma$ points $\Delta_{\mathrm{K} \Gamma}=\varepsilon_{\mathrm{K}}-\varepsilon_{\Gamma}$ in LDA and GGA for bulk and monolayer (ML) MoS 2 . A van der Waals functional should be used to obtain a reasonable interlayer separation for bulk layered $\mathrm{MoS}_{2}$. Because we are only interested in monolayers of $\mathrm{MoS}_{2}$ in this Chapter, we have used the experimental value of $c$ to obtain the bulk results shown here.

\begin{tabular}{lllllll}
\hline & & $a(\AA)$ & $d_{\mathrm{SS}}(\AA)$ & $d_{\mathrm{MoS}}(\AA)$ & $\Delta \varepsilon_{g}(\mathrm{eV})$ & $\Delta_{\mathrm{K} \Gamma}$ \\
\hline Bulk & GGA & 3.183 & 3.127 & 2.42 & 0.885 & -0.640 \\
& LDA & 3.125 & 3.115 & 2.38 & 0.748 & -0.640 \\
& Exp & $3.160^{a}$ & $3.172^{a}$ & $2.41^{a}$ & $1.290^{c}$ & $-0.600^{b}$ \\
ML & GGA & 3.185 & 3.130 & 2.42 & 1.650 & 0.012 \\
& LDA & 3.120 & 3.115 & 2.38 & 1.860 & 0.150 \\
& Exp & 3.160 & 3.172 & 2.41 & $1.900^{c}$ & $0.140^{b}$ \\
\hline
\end{tabular}

${ }^{a}$ Ref.113, ${ }^{b}$ Ref.114 ${ }^{c}$ Ref.11

The equilibrium structural parameters for bulk and monolayer $\mathrm{MoS}_{2}$ were calculated in both the LDA [115] and GGA [116] and are given in Table 3.1. It can be seen that the GGA slightly overestimates lattice constants and bond lengths compared to experiment [113]. The LDA underestimates them by more than the GGA overestimates them, a result found for many materials. In the present case, the agreement with experiment is still very reasonable for both LDA and GGA. However, we see that for an $\mathrm{MoS}_{2}$ monolayer the LDA gives a better description of the energy levels near the valence band maximum (VBM) than does the GGA, in particular the important quantity $\Delta_{\mathrm{K} \Gamma}=\varepsilon_{\mathrm{K}}-\varepsilon_{\Gamma}$, the position of the VBM at the $\Gamma$ point, $\varepsilon_{\Gamma}$, relative to the top of the valence band at the K point, $\varepsilon_{\mathrm{K}}$ [114]. To describe acceptor states accurately, it is important to have a good description of the host band structure in the vicinity of the VBM so we will describe exchange and correlation effects in this Chapter using the local spin density approximation LSDA as parameterized by Perdew and Zunger [115]. Results obtained with the GGA are considered in Sec. 3.5.

We model substitutional impurities and impurity pairs in $N \times N$ in-plane supercells with $N$ as large as 15 using the calculated equilibrium lattice constant for the pure monolayer (ML) host, Fig. 3.3. Local geometries are first relaxed using $N=6$ and only a small differential relaxation needs to be performed in the larger supercells. Interactions between pairs of impurities were studied in $12 \times 12$ supercells. The atomic positions were relaxed using a $2 \times 2 \times 1 \Gamma$-centered $k$-point mesh until 


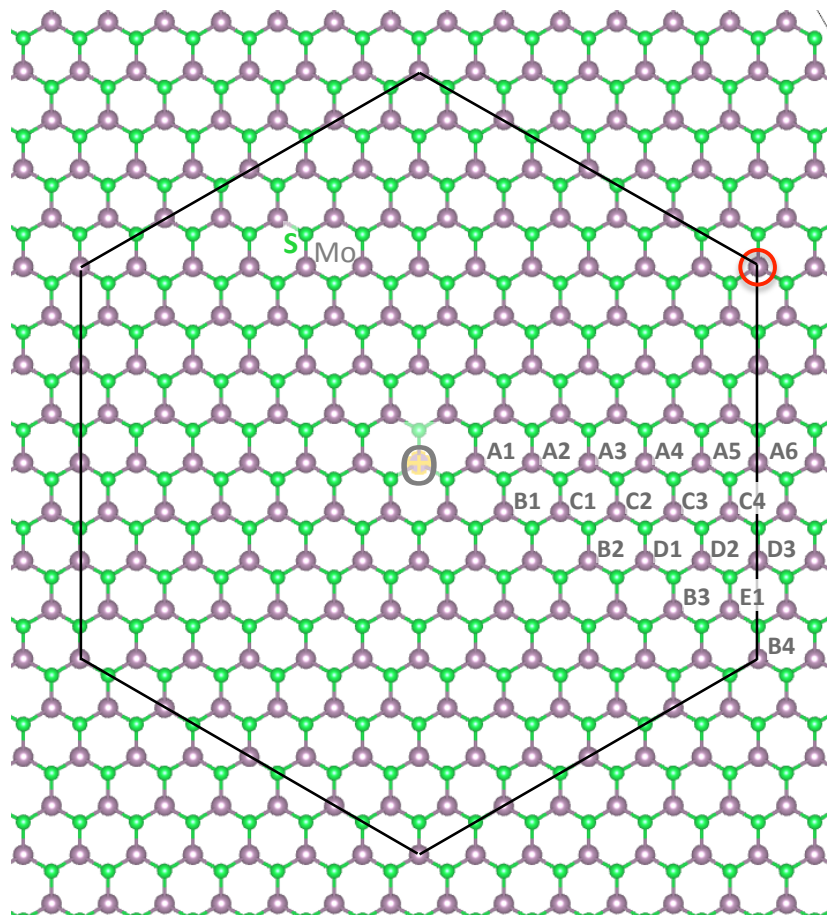

Figure 3.3: Sketch of a $12 \times 12 \mathrm{MoS}_{2}$ supercell with a substitutional atom at the origin, O. Shown is the more symmetric Wigner-Seitz cell. The potential on the Mo atom indicated with a red circle that is furthest from this atom will be used to identify the host valence band maximum (VBM). Mo atoms at various distances from the central atom are labelled A1-A6, B1-B4, C1-C4, D1-D3 and E1 for later reference.

the forces on each ion were smaller than $0.01 \mathrm{eV} / \AA$. Spin-polarized calculations were performed with a denser mesh corresponding to $4 \times 4 k$-points for a $12 \times 12$ unit cell.

\subsection{Results}

Impurity states in semiconductors are usually described in one of two limits: (i) in effective mass theory (EMT) where the main emphasis is on the Rydberg series of bound states tied to the conduction band minima or valence band maxima formed in response to a Coulomb potential or (ii) in the tight-binding limit where the main emphasis is on the local chemical binding, atomic relaxation and impurity states formed deep in the fundamental bandgap associated with an impurity potential very different to the host atomic potential $[117 ; 118 ; 119]$. Because there is no consensus of how best to combine both aspects [120], we consider the behaviour 


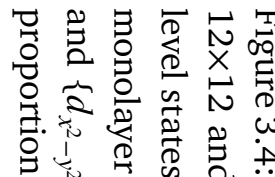

¿

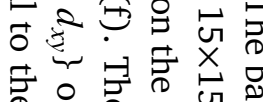

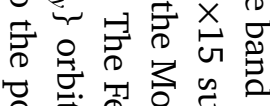

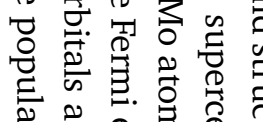

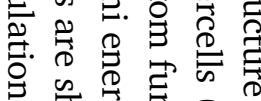

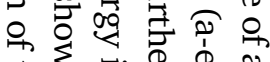

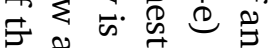

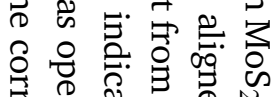

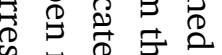

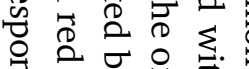

명요용

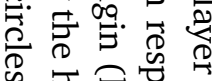

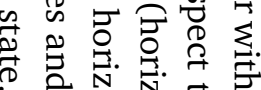

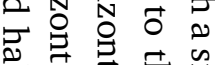

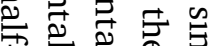

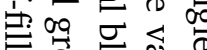

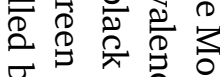

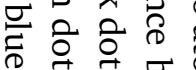

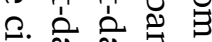

है है ڤँ

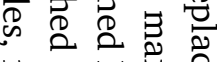

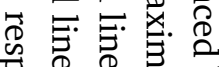

团莙声㲒

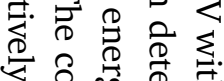

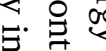

ㄹ. 궁.

仓苛. 它呑

$\sum$ 号范

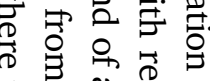

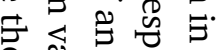

$\approx \cong \stackrel{2}{2}$

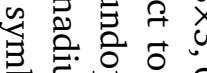

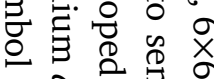

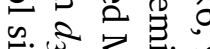
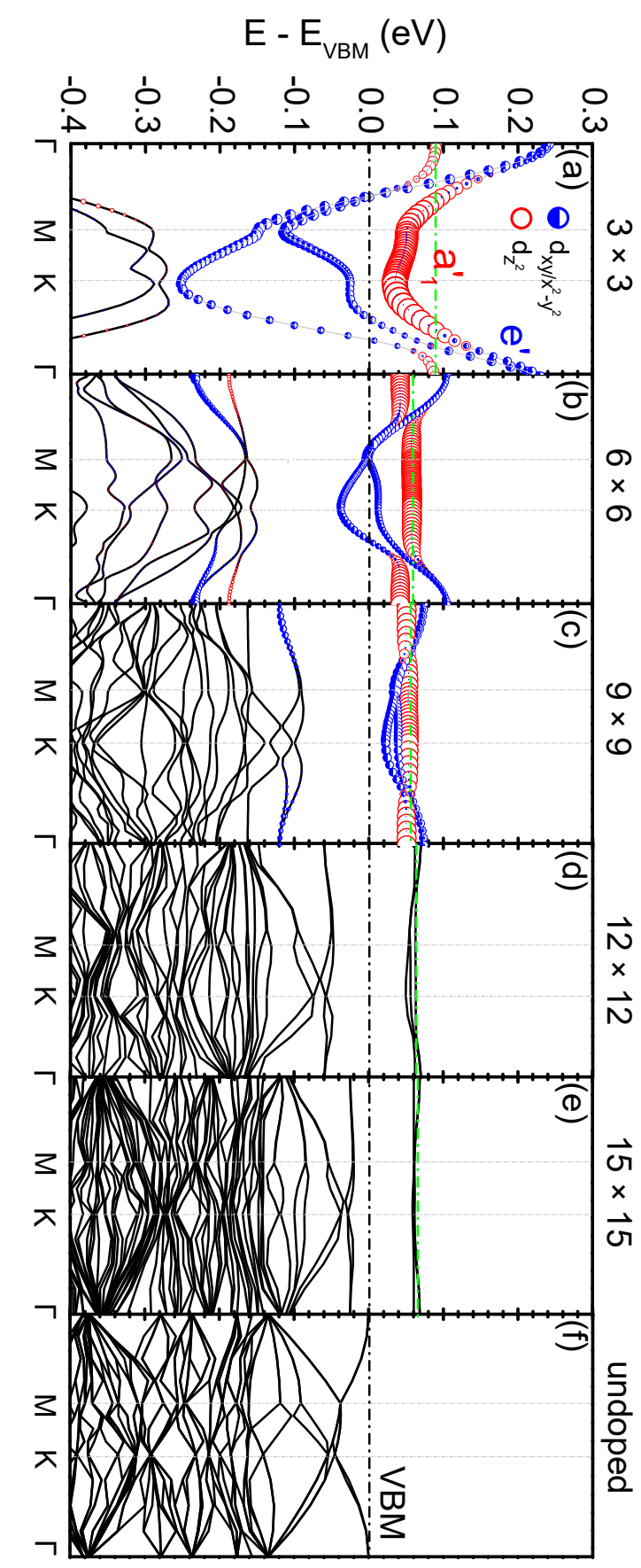

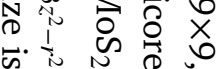


of shallow acceptor states in a periodic supercell geometry in some detail in the following section.

\subsubsection{Single impurity limit: $\mathrm{V}$ in $\mathrm{MoS}_{2}$}

We begin by replacing a single Mo atom in an $N \times N \mathrm{MoS}_{2}$ supercell with a $\mathrm{V}$ atom with one valence electron less, Fig. 3.3. To more easily identify the downfolded host bands we choose $N$ to be a multiple of three whereby the $\mathrm{K}$ and $\mathrm{K}^{\prime}$ points fold down to the $\Gamma$ point of the reduced BZ. The energy bands for this supercell before relaxing the local geometry are shown in Fig. 3.4 for $N=3,6,9,12$ and 15. The repulsive (for electrons; attractive for holes) impurity potential is seen to push not one but three impurity states out of the valence band to form localized states labeled $a_{1}^{\prime}$ and $e^{\prime}$ under the local $D_{3 h}$ symmetry, Fig. 3.2. By projecting the corresponding wavefunctions at the $\Gamma$ point onto spherical harmonics on the $V_{\text {Mo }}$ site, we find that the singly degenerate $a_{1}^{\prime}$ state has $\mathrm{V} d_{3 z^{2}-r^{2}}$ character while the $e^{\prime}$ state that is doubly degenerate at the center of the BZ has $\mathrm{V}\left\{d_{x^{2}-y^{2}}, d_{x y}\right\}$ character. The corresponding partial charge density plots are shown on the left- respectively right-hand sides (lhs, rhs) of Fig. 3.5. By fitting the wave functions of the impurity states to a hydrogenic wave function $\psi(r)=\operatorname{Aexp}\left(-r / a_{0}^{*}\right)$, we find effective Bohr radii $a_{0}^{*}$ of $4.2 \AA$ and $8.0 \AA$ for the $a_{1}^{\prime}$ and $e^{\prime}$ states, respectively in Fig. 3.5(c) and Fig. 3.5(d).

We identify these $a_{1}^{\prime}$ and $e^{\prime}$ states with the most tightly bound (effective mass like) acceptor states formed when a screened Coulomb potential is introduced by substitution of a Mo atom by $\mathrm{V}$ (Nb or $\mathrm{Ta}$ ). In the single impurity limit, intervalley scattering leads to a twofold orbitally degenerate effective mass like state formed from Mo $\left\{d_{x^{2}-y^{2}}, d_{x y}\right\}$ states bound to the $\mathrm{K}$ and $\mathrm{K}^{\prime}$ valence band maxima in Fig. 3.1 and a singly degenerate state with Mo $d_{3 z^{2}-r^{2}}$ character bound to the slightly lower lying valence band maximum at $\Gamma$ in Fig. 3.1. Within the accuracy of our calculations, these $e^{\prime}$ and $a_{1}^{\prime}$ states are (accidentally) degenerate for $\mathrm{MoS}_{2}$ and accommodate the hole that we will see polarizes fully in the local spin density approximation [121]. The shape of the dispersion of the impurity states is essentially independent of the supercell size so the bands can be described with a single effective hopping parameter. The $a_{1}^{\prime}$ state exhibits very little dispersion consistent with the out-of-plane $d_{3 z^{2}-r^{2}}$ orbital character at $\Gamma$ where the weak dispersion of the host $\mathrm{MoS}_{2}$ bands is described by a large effective mass [122]. In the language of effective mass theory (EMT), the binding energy of the $a_{1}^{\prime}$ state is dominated by the central cell correction [117].

In the rightmost panel of Fig. 3.4, we show the band structure of an undoped monolayer calculated in a $15 \times 15$ supercell so the $\mathrm{K}$ point VBM is downfolded onto $\Gamma$. If we compare this with the impurity supercell bands on the left, we see that even for $N=15$, Fig. 3.4(e), the interaction of the impurity bands and the VBM still suppresses the VBM quite noticeably, by more than $20 \mathrm{meV}$. 
(a) Top view: [001] plane

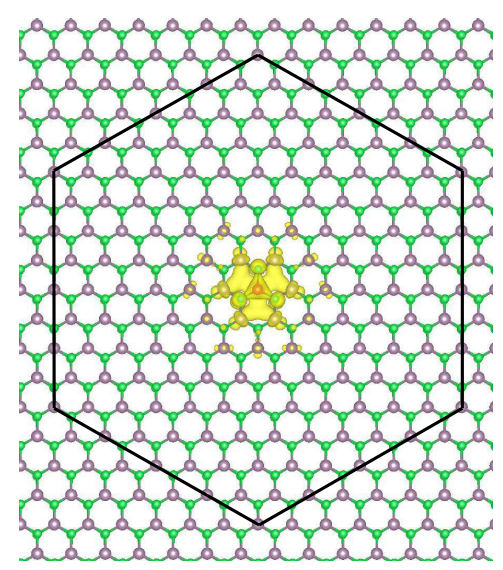

Side view: [010] plane

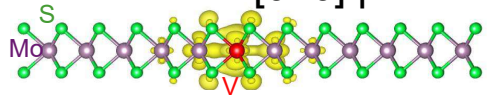

(c)

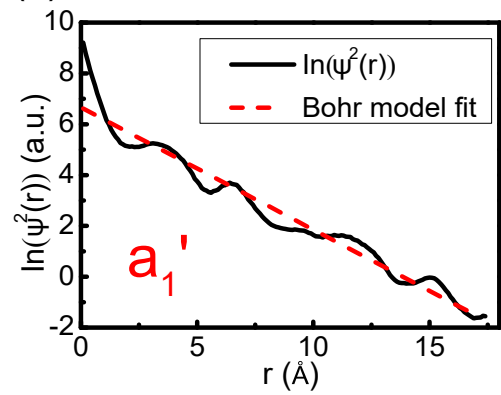

(b) Top view: [001] plane

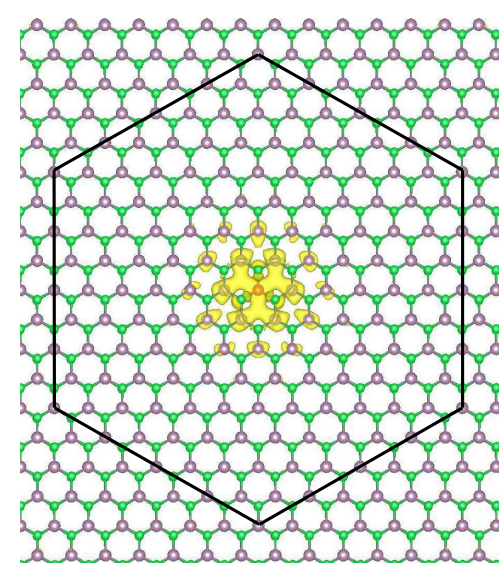

Side view: [010] plane

(d)

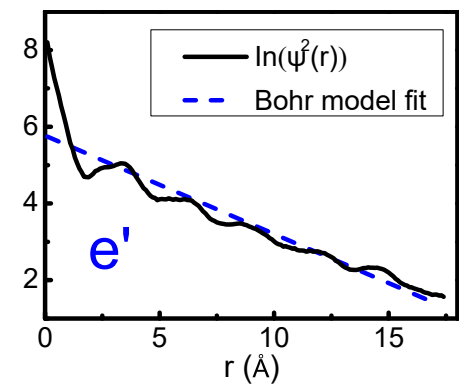

Figure 3.5: Charge density plots for the $a_{1}^{\prime}$ (lhs) and $e^{\prime}$ (rhs) states at the $\Gamma$ point in Fig. 3.4(d) in the central [001] plane through the Mo atoms (top view) and [010] plane (side view) for a $12 \times 12$ supercell. The isosurface levels are $0.001 e / \AA^{3}$. (c) and (d): circularly averaged charge densities fitted with a Bohr model.

\section{Screened impurity potential}

Identifying the valence band maximum (VBM) in an impurity supercell calculation is complicated by the Rydberg series of effective mass like states associated with the single impurity whose wavefunctions will overlap with their periodic images and form bands that overlap and hybridize with the "true" valence band states, Fig. 3.2. To disentangle those effects, we first determine the position of the VBM with respect to Mo $4 s$ semicore states, $\varepsilon_{4 s}^{\mathrm{Mo}}$, for an undoped monolayer of $\mathrm{MoS}_{2}$; $\varepsilon$ denotes a Kohn-Sham eigenvalue. For a sufficiently large impurity supercell, the position of $\varepsilon_{4 s}^{\text {Mo }}$ for the Mo atom furthest from the impurity (indicated with a 


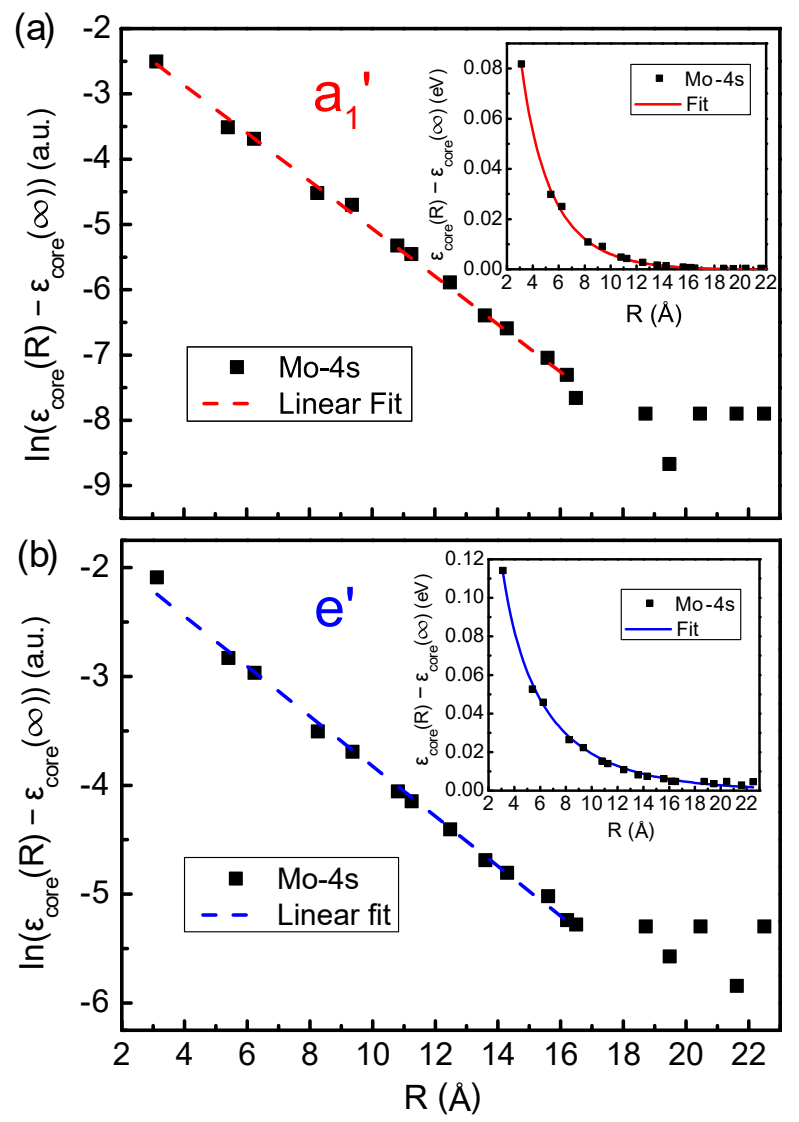

Figure 3.6: Dependence of the Mo $4 s$ semicore level on the separation from the $\mathrm{V}_{\mathrm{Mo}}$ dopant ion. The Coulomb potential of the $\mathrm{V}$ dopant is screened by the host valence electrons and by the $a_{1}^{\prime}$ hole (upper panel) respectively by the $e^{\prime}$ hole (lower panel). The 18 data points refer to the 18 inequivalent Mo atoms labelled in Fig. 3.3. The asymptotic value $\varepsilon_{\text {core }}(\infty)$ was determined by fitting the calculated data points in the insets to an exponential wave function and using this fit (red and blue curves) to extrapolate to $R=\infty$.

red circle in Fig. 3.3) relative to the VBM should be asymptotically equal to the corresponding energy separation for an undoped monolayer of $\mathrm{MoS}_{2}$ because the impurity potential far from the dopant center will be completely screened by the bound charge of the neutral impurity in an $a_{1}^{\prime}$ or $e^{\prime}$ bound state. To test this hypothesis quantitatively, we plot $\varepsilon_{4 s}^{\mathrm{Mo}}$ with respect to its asymptotic value as a function of the separation of Mo from the impurity V ion in the insets of Fig. 3.6 for $N=12$ supercells (symbols). The corresponding results for the $\mathrm{S}$ semicore $2 s$ state $\varepsilon_{2 p}^{\mathrm{S}}$ are shown in Appendix 3.8.1 and yield similar conclusions.

In an impurity supercell calculation, a localized electron in a (semi)core level on 
a Mo atom a distance $R$ from the impurity atom will see a screened $1 / \epsilon_{r} R$ repulsive potential that is partially compensated by the charge of the bound hole, $n_{\text {hole }}(r)$. Here $\epsilon_{r}$ is the relative static dielectric constant. We can "measure" this screened Coulomb potential by studying how Mo (and S) semicore levels behave as a function of their separation from the central $\mathrm{V}$ atom. The perturbing electrostatic potential seen by the core electrons has the form

$$
\begin{aligned}
\varepsilon_{\text {core }}(R)-\varepsilon_{\text {core }}(\infty) & =\frac{1}{\epsilon_{r} R}\left(1-\int_{0}^{R} n_{\text {hole }}(r) 2 \pi r d r\right) \\
& =\frac{e^{\frac{-2 R}{a_{0}^{*}}}\left(\frac{2 R}{a_{0}^{*}}+1\right)}{\epsilon_{r} R}
\end{aligned}
$$

where in (3.1a) $n_{\text {hole }}(r)=\int_{-\infty}^{\infty} n_{\text {hole }}(r, z) d z$ and $n_{\text {hole }}(r, z)$ is obtained by integrating $\left|\psi_{i}(r, \theta, z)\right|^{2}$ over $\theta$ for $i=a_{1}^{\prime}$ or $e^{\prime}$. In (3.1b), we assume that $\psi_{i}(r, \theta, z)$ is the solution of a strictly two-dimensional hydrogenic problem [123]. If we take the natural logarithm of (3.1b), the slope is $-2 / a_{0}^{*}$ for large values of $R$ and we can extract $a_{0}^{*}$ from Fig. 3.6.

In the supercell band structures shown in Fig. 3.4, the $a_{1}^{\prime}$ and $e^{\prime}$ derived states overlap and $n_{\text {hole }}(r)$ is a mixture of these two states with different masses $m_{h}$. To circumvent this complication, we calculate the electronic structure at the $\mathrm{K}$ (or M) point where the lowest unoccupied state has $a_{1}^{\prime}$ character. By using a sufficiently small temperature broadening we can obtain the corresponding charge density and obtain the result shown in Fig. 3.6 (upper panel). Alternatively, we calculate the electronic structure at the $\Gamma$ point where the lowest unoccupied state has $e^{\prime}$ character to obtain Fig. 3.6 (lower panel). The ab-initio values of $\varepsilon_{\text {core }}(R)$ and $\ln \left[\varepsilon_{\text {core }}(R)-\varepsilon_{\text {core }}(\infty)\right]$ are fit quite well with (3.1b) with an effective Bohr radius of $a_{0}^{*} \sim 5.4 \AA$ and $\epsilon_{r}=20$ for the $a_{1}^{\prime}$ hole and $a_{0}^{*} \sim 8.7 \AA$ and $\epsilon_{r}=13$ for the $e^{\prime}$ hole. The values of $\epsilon_{r}$ should be compared to recent calculations for the in-plane "macroscopic" dielectric constant where $\epsilon_{r}=15$ was found for monolayers of $\mathrm{MoS}_{2}$ as well as for bulk $\mathrm{MoS}_{2}$ with negligible ionic contribution to the screening [124]. The deviation of $\epsilon_{r}=20$ from the macroscopic value is not very surprising in view of the localization of the $a_{1}^{\prime}$ hole that does not "see" many unit cells of $\mathrm{MoS}_{2}$. The value for the $e^{\prime}$ hole is reasonable.

At large values of $R$ in Fig. 3.6, the potential felt by the core states is seen not to decay but to oscillate. We attribute this to the accumulation of the residual hole charge at the supercell boundary that is a consequence of charge neutrality. The data points in Fig. 3.6 that deviate from the trend line are to be found outside the circle inscribed in the hexagonal WS cell.

In the effective mass approximation the effective Bohr radius $a_{0}^{*}=\epsilon_{r} / m_{h} \times 0.529$ $\AA$ and the ground state binding energy with respect to the appropriate VBM is $\varepsilon_{b}=m_{h} / \epsilon_{r}^{2} \times 13.606 \mathrm{eV}$. From the band structure in Fig. 3.1, the effective mass in 
units of the free electron mass $m_{0}$ is $m_{h} \sim 0.56$ at the $\mathrm{K}$ point VBM and 3.42 at the $\Gamma$ point VBM, consistent with a previous calculation [125]. Combining these masses with $\epsilon_{r}=15$ [124] leads to values of $a_{0}^{*} \sim 14 \AA$ and $\varepsilon_{b} \sim 34 \mathrm{meV}$ for $e^{\prime}$ holes and $a_{0}^{*} \sim 2.3 \AA$ and $\varepsilon_{b} \sim 207 \mathrm{meV}$ for $a_{1}^{\prime}$ holes, respectively. At best the EMT is indicative but is clearly not quantitative for the most strongly bound acceptor states - a conclusion that is not especially surprising in view of the expected central cell correction for ground states [117] as well as the strong localization of both states.

The screening of the impurity potential by (i) the $\mathrm{MoS}_{2}$ valence electrons and (ii) by the bound impurity hole means that the residual perturbation measured by the core states decreases rapidly with $R$ allowing us to estimate the position of the reference core state far from the impurity and therefore of the VBM to an accuracy of a few meV for $N=12$. This procedure was used to estimate the position of the VBM and of the impurity states with respect to it for each supercell size shown in Fig. 3.4 (dot-dashed line).

The same results can be obtained more simply by noting that the repulsive potential that binds a Rydberg series to the top of the valence band has little effect on the conduction band edge. Since we know the value of the band gap, the VBM can be determined from the conduction band minimum. We verified that this leads to the same results as the more elaborate procedure discussed in the foregoing.

\section{Hydrogenic perturbation model}

Now that we have established procedures for determining the position of the VBM, we see that the $a_{1}^{\prime}$ and $e^{\prime}$ impurity bands in Fig. 3.4 not only narrow as the supercell size $N$ is increased but rise with respect to the VBM. To make this clearer, we plot their centers of gravity

$$
\bar{\varepsilon}_{i}=\frac{\sum_{n \mathbf{k}} f_{i n}(\mathbf{k}) \varepsilon_{n}(\mathbf{k})}{\sum_{n \mathbf{k}} f_{i n}(\mathbf{k})}
$$

with respect to the VBM in Fig. 3.7; both levels are seen to rise as a function of $N$. The probability $f_{i n}(\mathbf{k})$ is the $i$ character of the wavefunction $\psi_{n \mathbf{k}}$ obtained by projecting $\psi_{n \mathbf{k}}$ onto site centered orbitals $\beta_{i}$ and $i \equiv R l m$ is a composite site, angular momentum index. Here we have chosen $i$ to be the $d_{x^{2}-y^{2}}$ and $d_{x y}$ Kubic harmonics on the $\mathrm{V}_{\text {Mo }}$ atom for the $e^{\prime}$ state and $d_{3 z^{2}-r^{2}}$ on $\mathrm{V}_{\text {Mo }}$ for the $a_{1}^{\prime}$ state and the summation is carried out over the entire Brillouin zone and over the three split off impurity bands in Fig. 3.4. Since the position of the impurity levels introduced by $\mathrm{V}_{\mathrm{Mo}}$ atoms will play an important role in determining the magnetic moment and exchange interaction between impurities, we wish to understand this increase.

A Coulomb potential in a semiconductor gives rise to a Rydberg series of bound states. A finite supercell cannot describe the asymptotic form of the potential correctly but will truncate it on the supercell boundary. In a self-consistent calculation, 


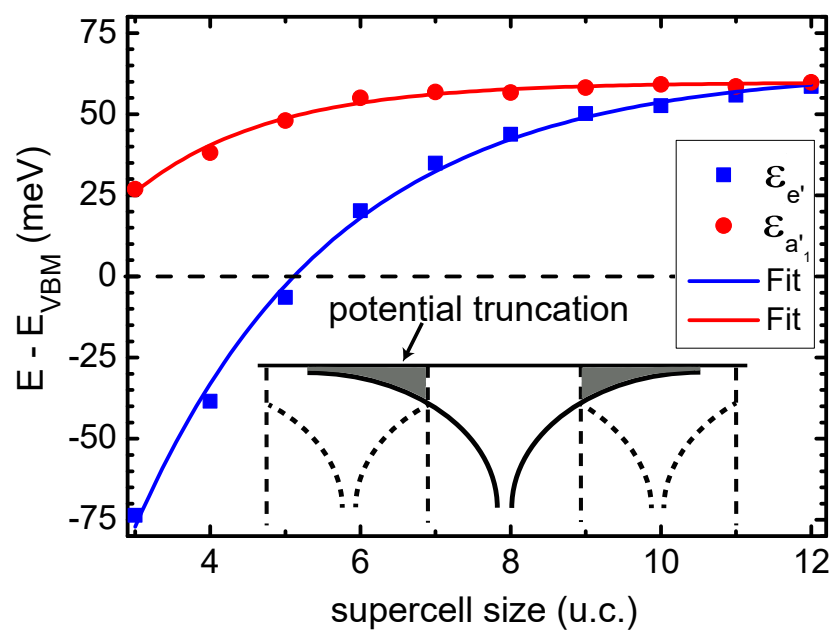

Figure 3.7: Dependence on the supercell size $N$ of the (spin-degenerate) $a_{1}^{\prime}$ and $e^{\prime}$ impurity levels induced by a substitutional vanadium atom $\mathrm{V}_{\text {Mo }}$ with respect to the valence band maximum. The solid lines are fits to the data points using a model that includes the tail of the Coulomb potential in first order perturbation theory. (Inset) Truncation of the Coulomb potential in a supercell calculation.

the requirement of charge neutrality will lead to the charge in the tail of the hydrogenic state accumulating on the supercell boundary. As the supercell size is increased, more of the "tail" of the (repulsive) Coulomb potential is described correctly, leading to the rise of the impurity levels seen in Figs. 3.4 and 3.7.

The effect of truncating the Coulomb potential can be estimated using a simple two-dimensional (2D) hydrogenic [123] model and first order perturbation theory. For simplicity we assume a circular geometry and replace the 2D Wigner-Seitz cell with a circle of radius $S$ with the same area $\pi S^{2}=A_{\text {ws }}$. The correction to the ground state energy of a hydrogen atom in $2 \mathrm{D}$ is

$$
\int_{S}^{\infty} R^{2}(r) \frac{e^{2}}{4 \pi \epsilon_{r} r} 2 \pi r d r=\frac{e^{2}}{2 \pi \epsilon_{r} a_{0}^{*}} e^{-2 S / a_{0}^{*}}
$$

where $\mathrm{a}_{0}^{*}$ is the effective Bohr radius, $\epsilon_{r}$ is the relative dielectric constant and $R(r)$ is the radial part of the $2 \mathrm{D}$ hydrogenic wave function [123] for a screened Coulomb potential. Taking the top of the valence band $\varepsilon_{\mathrm{VBM}}$ of an ideal $\mathrm{MoS}_{2}$ monolayer, as estimated in the previous subsection, to be zero, we fit the ab-initio calculated data points with the solid curves shown in Fig. 3.7. The fit is very good and deviations can be attributed to local screening effects in the "real" inhomogeneous crystal as modelled in DFT.

The $a_{1}^{\prime}$ and $e^{\prime}$ impurity levels increase in energy with increasing supercell size and converge to a (coincidentally) common value of $\sim 62 \mathrm{meV}$ in the single impurity 
limit $(N \rightarrow \infty)$. From the fitting, we obtain another estimate of the effective Bohr radius of $8.3 \AA$ for the $e^{\prime}$ state, of $5.5 \AA$ for the $a_{1}^{\prime}$ state and of $\epsilon_{r} \sim 10.0$ for the inplane dielectric constant. These values should be compared to the EMT predictions of $a_{0}^{*} \sim 14 \AA$ and $\varepsilon_{b} \sim 34 \mathrm{meV}$ with respect to the K point VBM for the $e^{\prime}$ holes and $a_{0}^{*} \sim 2.3 \AA$ and $\varepsilon_{b} \sim 207 \mathrm{meV}$ with respect to the $\Gamma$ point VBM for the $a_{1}^{\prime}$ holes. Taking the LDA value of $\Delta_{\mathrm{K} \Gamma} \sim 150 \mathrm{meV}$ from Table 3.1 into account, we would expect to find the $a_{1}^{\prime}$ ground state at $207-150=57 \mathrm{meV}$ above the K point VBM.

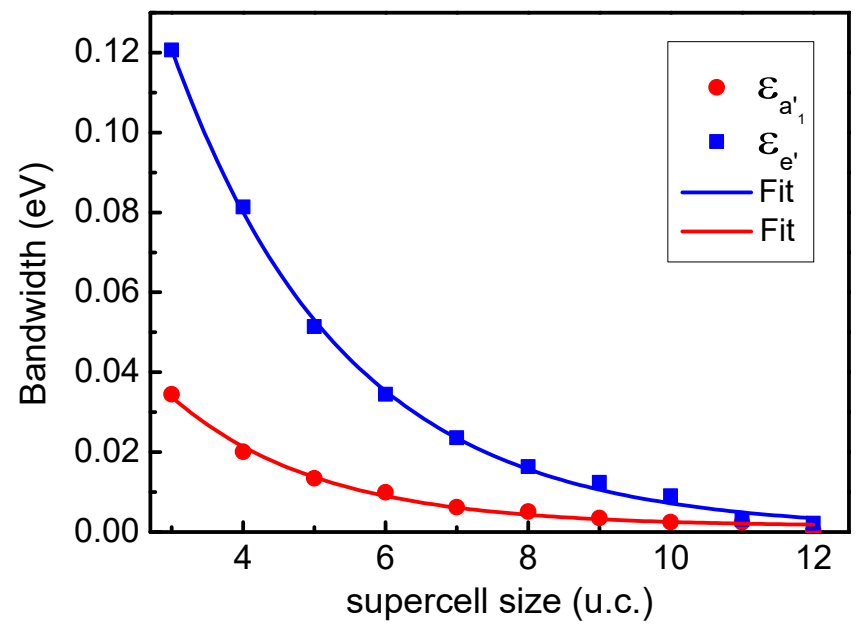

Figure 3.8: Dependence of the band width of (spin-degenerate) impurity bands induced by substitutional $\mathrm{V}$ impurities on the supercell size $N$. The data points calculated using (3.4) are fit using the hydrogen model discussed in the text.

According to Fig. 3.7, the highest $e^{\prime}$ impurity states emerge from the valence band when the supercell size is larger than $5 \times 5$. This is consistent with the effective Bohr radius of the impurity levels deduced in Fig. 3.5. For impurity states with higher principal quantum numbers, the Bohr radii are at least twice as large. These states are not sufficiently localized in the Coulomb potential to appear above the valence band maximum for the largest supercells we have studied.

We can also determine effective Bohr radii from the dependence of the widths of the impurity bands, shown in Fig. 3.4, on the supercell size $N$ because of the dependence of the bandwidth on the overlap of impurity wavefunctions in neighboring supercells. In Fig. 3.8 we plot the second moment of the impurity bands

$$
\sqrt{\frac{\sum_{n \mathbf{k}} f_{i n}(\mathbf{k})\left(\varepsilon_{n}(\mathbf{k})-\bar{\varepsilon}_{i}\right)^{2}}{\sum_{n \mathbf{k}} f_{i n}(\mathbf{k})}} \propto e^{-R / a_{0}^{*}}
$$

as a function of $N$ where $\mathrm{a}_{0}^{*}$ is the effective Bohr radius and $R$ is the distance between dopants in neighboring supercells. From the fitting, we get effective Bohr radii of 
$7.8 \AA$ for the $e^{\prime}$ state and $5.2 \AA$ for the $a_{1}^{\prime}$ state which are consistent with our earlier results summarized in Table 3.2.

Table 3.2: Summary of the $a_{1}^{\prime}$ and $e^{\prime}$ bound state effective Bohr radii $a_{0}^{*}(\AA)$ derived in different ways without relaxation.

\begin{tabular}{|c|c|c|c|c|c|c|c|}
\hline & & \multicolumn{2}{|c|}{$\mathrm{V}$} & \multicolumn{2}{|c|}{$\mathrm{Nb}$} & \multicolumn{2}{|c|}{$\mathrm{Ta}$} \\
\hline & & $a_{1}^{\prime}(\Gamma)$ & $e^{\prime}(\mathrm{K})$ & $a_{1}^{\prime}(\Gamma)$ & $e^{\prime}(\mathrm{K})$ & $a_{1}^{\prime}(\Gamma)$ & $e^{\prime}(\mathrm{K})$ \\
\hline$\overline{\text { Fig. } 3.5}$ & & 4.2 & 8.0 & 5.3 & 10.0 & 5.2 & 10.3 \\
\hline Fig. 3.6, & Eq. $3.1 \mathrm{~b} \mathrm{Mo}$ & 5.4 & 8.7 & 6.2 & 9.6 & 5.8 & 9.7 \\
\hline Fig. 3.29, & Eq. 3.1b S & 6.5 & 8.8 & 6.8 & 10.0 & 6.5 & 10.0 \\
\hline Fig. 3.7, & Eq. 3.3 & 5.5 & 8.3 & 5.9 & 10.0 & 6.2 & 10.4 \\
\hline Fig. 3.8, & Eq. 3.4 & 5.2 & 7.8 & 5.2 & 9.8 & 5.4 & 10.1 \\
\hline EMT & $\left(\epsilon_{r}=15\right)$ & 2.3 & 14.0 & 2.3 & 14.0 & 2.3 & 14.0 \\
\hline
\end{tabular}

\section{Effect of relaxation}

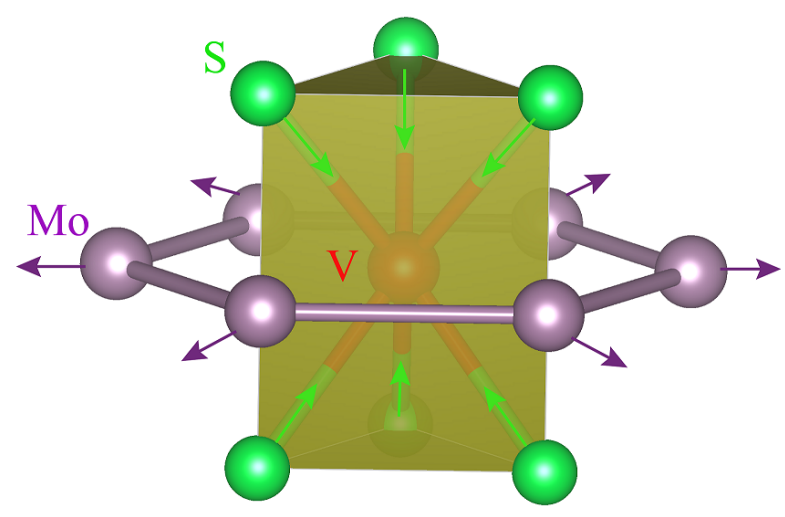

Figure 3.9: (color online). Schematic of the relaxation about a vanadium atom on a substitutional Mo site, $\mathrm{V}_{\mathrm{Mo}}$, in $\mathrm{MoS}_{2}$.

One of the most attractive and useful features of a plane wave basis is the ease with which Hellmann-Feynman forces can be calculated. This makes it simple to determine how the host $\mathrm{MoS}_{2}$ crystal relaxes locally in response to substituting a Mo atom with V, Fig. 3.9. According to the electronic structure Fig. 3.4(d) for the unrelaxed geometry, shown enlarged in Fig. 3.10(b), the Fermi level is essentially pinned in the orbitally nondegenerate $a_{1}^{\prime}$ state and the system does not undergo a Jahn-Teller (JT) distortion; if we begin geometry optimisation from a JT distorted configuration, the system relaxes back to a symmetric one. Consistent with this, we 
find only symmetry-conserving ("breathing mode") relaxation about the vanadium ion with the six nearest neighbour sulphur atoms relaxing towards the $\mathrm{V}$ atom and the six in-plane neighbouring Mo atoms relaxing radially away, shown in Fig. 3.9. The displacements converge rapidly with supercell size to $\Delta d_{\mathrm{V}-\mathrm{S}}=0.060 \AA$ and $\Delta d_{\mathrm{V}-\mathrm{Mo}}=0.008 \AA$ as seen in Table 3.3. The total energy gain from relaxation is $150 \mathrm{meV}$.

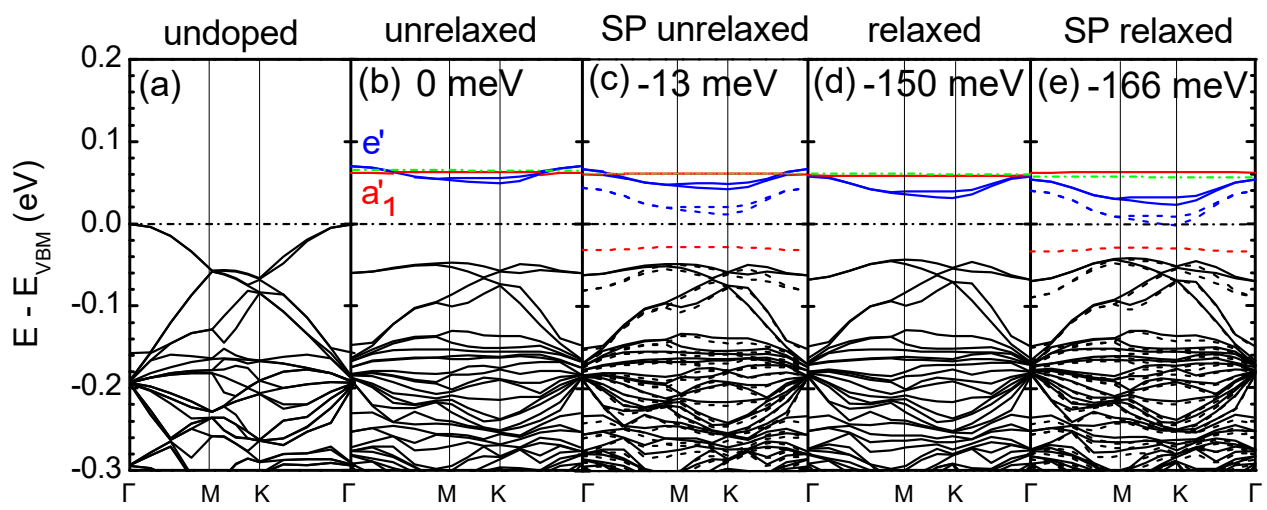

Figure 3.10: Effect of relaxation and spin-polarization on the electronic structure of a $12 \times 12$ supercell for an $\mathrm{MoS}_{2}$ monolayer with a single Mo atom replaced by V. (a) Reference bands for an undoped $\mathrm{MoS}_{2}$ monolayer, energy bands for a single substitutional V impurity (b) without relaxation, (c) with spin polarization (SP) and without relaxation, (d) with relaxation, without spin polarization, (e) spin polarized and relaxed. The $a_{1}^{\prime}$ level is red, the $e^{\prime}$ states are blue. In (c) and (e), the solid (dashed) lines indicate minority (majority) spin states. The energy gain with respect to the unrelaxed case is given in each panel in meV. The zero of energy is the VBM and the Fermi level is indicated by a green dot-dashed line.

The band structures of the unrelaxed and relaxed $12 \times 12$ impurity supercells are compared in panels (b) and (d) of Fig. 3.10. The band structures are aligned on the VBM, located at the $\Gamma$ point as seen in Fig. 3.10(a) for an undoped monolayer, using the $4 s$ semicore level shift of the "B4" Mo atom on the boundary of the Wigner-Seitz cell furthest from the dopant $\mathrm{V}$ atom, see Fig. 3.3. The main effect of relaxation is to lift the quasidegeneracy of the $a_{1}^{\prime}$ and $e^{\prime}$ impurity states, Fig. 3.10(d). The increased V-Mo bond length leads to a lowering of the center of gravity of the $e^{\prime}$ state with respect to the $a_{1}^{\prime}$ state that is antibonding with respect to the neighbouring $\mathrm{S} p$ states. As a consequence, the hole state acquires essentially pure $a_{1}^{\prime}$ character. 
Table 3.3: Relaxation of nearest neighbour $\mathrm{S}$ and Mo shells about a substitutional vanadium atom as a function of the $N \times N$ supercell size $N$. Atomic displacmenents in $\AA$.

\begin{tabular}{lccccc}
\hline$N$ & 3 & 6 & 9 & 12 & 15 \\
\hline$\Delta d_{\mathrm{V}-\mathrm{S}}$ & -0.060 & -0.060 & -0.060 & -0.060 & -0.060 \\
$\Delta d_{\mathrm{V}-\mathrm{Mo}}$ & 0.003 & 0.005 & 0.008 & 0.008 & 0.008 \\
\hline
\end{tabular}

\section{Spin polarization}

The electronic structure of an unpaired spin in an orbitally nondegenerate $a_{1}^{\prime}$ impurity state resembles that of a free hydrogen-like atom and, like a free atom, its total energy can be lowered by allowing the electron to polarize in the local spin density approximation $[121 ; 126]$. The result of doing so in the dilute limit is shown in Figs. 3.10(c) and (e). Without relaxation, the localized and dispersionless $a_{1}^{\prime}$ state splits by $91 \mathrm{meV}$ leaving the hole with mixed $a_{1}^{\prime}-e^{\prime}$ character and a magnetic moment of $m=1 \mu_{B}$. Expressing the exchange splitting in terms of an effective Stoner parameter $I_{\mathrm{xc}}$ as $\Delta \varepsilon=m I_{\mathrm{xc}}$ results in a value of $I_{\mathrm{xc}}$ of $91 \mathrm{meV}$ that is substantially less than the free atom value of $I_{\mathrm{xc}} \sim 0.7 \mathrm{eV}$ [127]. In the local density approximation, it is the local electron density that drives the exchange splitting and the small exchange splitting can be understood in terms of the much lower spin density of the impurity state compared to that of a free atom. Consistent with this picture is the even smaller exchange splitting of the more delocalized $e^{\prime}$ state that is only $\sim 25 \mathrm{meV}$. Before relaxation, the partial occupation of $a_{1}^{\prime}$ and $e^{\prime}$ states allows the $e^{\prime}$ state to "freeload" on the much more localized $a_{1}^{\prime}$ electron density enhancing its spin polarization and exchange splitting $(24.7 \mathrm{meV})$ which decreases to $15.2 \mathrm{meV}$ after relaxation, Table 3.6. The mixing of the $e^{\prime}$ impurity state with what will eventually become the top of the valence band is clearly seen in the $12 \times 12$ supercell in terms of the large exchange splitting of the uppermost host valence band state (black solid and dashed bands) at the $\Gamma$ point. The total energy gain from spin polarization of $13 \mathrm{meV}$ without relaxation or $16 \mathrm{meV}$ with relaxation is dwarfed by the $150 \mathrm{meV}$ energy gain from relaxation.

For smaller supercell sizes, the dispersion of the $e^{\prime}$ state increases until it overlaps the unoccupied $a_{1}^{\prime}$ level and begins to quench the spin polarization for $N<8$, Table 3.4. Reducing the supercell size further increases the quenching and when, in addition, the impurity potential fails to pull the impurity levels above the VBM for supercell sizes smaller than $5 \times 5$, the magnetic moment disappears. Relaxation enhances the magnetic moment by reducing the overlap of the $a_{1}^{\prime}$ and $e^{\prime}$ states in spite of the unfavourable increase of the $e^{\prime}$ state dispersion. Even a very small value of the Coulomb repulsion parameter $U=1 \mathrm{eV}$ [128] can lead to a $3 \times 3$ supercell becoming polarized. Most of the discrepancies in the literature can be explained 
Table 3.4: Calculated magnetic moment (in $\mu_{B}$ ) for an $\mathrm{MoS}_{2}$ monolayer supercell doped with $\mathrm{V}, \mathrm{Nb}$ and $\mathrm{Ta}$ as a function of the $N \times N$ supercell size without (Un) and with (Re) relaxation. For $\mathrm{V}$ the effect of an onsite Coulomb repulsion parameter $U=1 \mathrm{eV}$ was examined for the relaxed case. The reciprocal space sampling density is constant.

\begin{tabular}{llcccccccccc}
\hline$N$ & & 3 & 4 & 5 & 6 & 7 & 8 & 9 & 10 & 11 & 12 \\
\hline $\mathrm{V}$ & $\mathrm{Un}$ & 0.00 & 0.00 & 0.61 & 0.82 & 0.97 & 1.00 & 1.00 & 1.00 & 1.00 & 1.00 \\
& $\mathrm{Re}$ & 0.00 & 0.00 & 0.81 & 1.00 & 1.00 & 1.00 & 1.00 & 1.00 & 1.00 & 1.00 \\
& $\mathrm{U}$ & 0.54 & 0.68 & 1.00 & 1.00 & 1.00 & 1.00 & 1.00 & 1.00 & 1.00 & 1.00 \\
\hline $\mathrm{Nb}$ & $\mathrm{Un}$ & 0.00 & 0.00 & 0.00 & 0.34 & 0.54 & 0.68 & 0.87 & 0.93 & 1.00 & 1.00 \\
& $\mathrm{Re}$ & 0.00 & 0.00 & 0.00 & 0.66 & 0.75 & 0.89 & 1.00 & 1.00 & 1.00 & 1.00 \\
\hline $\mathrm{Ta}$ & $\mathrm{Un}$ & 0.00 & 0.00 & 0.00 & 0.32 & 0.42 & 0.57 & 0.86 & 0.95 & 1.00 & 1.00 \\
& $\mathrm{Re}$ & 0.00 & 0.00 & 0.00 & 0.68 & 0.83 & 0.92 & 1.00 & 1.00 & 1.00 & 1.00 \\
\hline
\end{tabular}

in terms of the supercell size, k-point sampling, exchange-correlation potential, $U$ etc. $[87 ; 90 ; 93 ; 96 ; 82 ; 98 ; 103 ; 104 ; 129]$

\section{Formation energies}

Table 3.5: Formation energies in eV of substitutional $\mathrm{V}, \mathrm{Nb}$ and $\mathrm{Ta}$ impurities in an $\mathrm{MoS}_{2}$ monolayer for a $12 \times 12$ supercell.

\begin{tabular}{lccc}
\hline & $\mathrm{V}$ & $\mathrm{Nb}$ & $\mathrm{Ta}$ \\
\hline Unrelaxed & 0.40 & 0.01 & -0.12 \\
Relaxed & 0.25 & -0.15 & -0.23 \\
\hline
\end{tabular}

The formation energy of a substitutional dopant $\mathrm{X}_{\mathrm{Mo}}$ is defined as

$$
E_{\text {form }}\left[\mathrm{X}_{\mathrm{Mo}}\right]=E_{\mathrm{tot}}\left[\mathrm{MoS}_{2}: \mathrm{X}\right]-E_{\mathrm{tot}}\left[\mathrm{MoS}_{2}\right]+\mu_{\mathrm{Mo}}-\mu_{\mathrm{X}}
$$

where $\mathrm{E}_{\text {tot }}\left[\mathrm{MoS}_{2}: \mathrm{X}\right]$ is the total energy of an $\mathrm{MoS}_{2}$ monolayer with one Mo atom replaced by one $\mathrm{X}$ atom, $\mathrm{E}_{\mathrm{tot}}\left[\mathrm{MoS}_{2}\right]$ is the total energy of a pristine $\mathrm{MoS}_{2}$ monolayer and $\mu_{\mathrm{Mo}}$ and $\mu_{\mathrm{X}}$ are the total energies per atom of Mo and $\mathrm{X}$ in their bulk metallic bcc phases, respectively. Taking the (spin-polarized) $S_{2}$ molecule as the reference chemical potential for $\mathrm{S}$, the heat of formation of a $\mathrm{MoS}_{2}$ monolayer, $E_{\text {form }}\left[\mathrm{MoS}_{2}\right]$, was calculated to be $-5.31 \mathrm{eV} /$ formula unit. The formation energy of $\mathrm{V}_{\mathrm{Mo}}$ is small and those of $\mathrm{Nb}_{\mathrm{Mo}}$ and $\mathrm{Ta}_{\mathrm{Mo}}$ actually become negative when relaxed indicating that doping $\mathrm{MoS}_{2}$ with these group $\mathrm{V}$ elements should be experimentally feasible, Table 3.5. 


\subsubsection{Binding of $\mathrm{V}$ impurity pairs}

Two substitutional dopant $\mathrm{V}$ atoms will have a negligible interaction energy when sufficiently far apart. This energy can be calculated as follows. First define a reference energy $E_{\mathrm{V}}^{N}$ for a single $\mathrm{V}$ dopant atom substituting a Mo atom in $\mathrm{MoS}_{2}$ as

$$
E_{\mathrm{V}}^{N}=E_{\mathrm{tot}}^{N}\left[\mathrm{~V}_{\mathrm{Mo}}\right]-E_{\mathrm{tot}}^{N}\left[\mathrm{MoS}_{2}\right]
$$

where $E_{\text {tot }}^{N}\left[\mathrm{MoS}_{2}\right]$ is the total energy of an $N \times N$ supercell of $\mathrm{MoS}_{2}$ in equilibrium and $E_{\mathrm{tot}}^{N}\left[\mathrm{~V}_{\mathrm{Mo}}\right]$ is the total energy of the same supercell with one Mo atom replaced with a V atom. To calculate absolute formation energies, suitable chemical potentials would need to be included to take account of where the $\mathrm{V}$ atom came from and where the Mo atom went to; we will not be concerned with those here. The binding energy $E_{b}$ is then

$$
E_{b}^{N}(R)=E_{\mathrm{tot}}^{N}\left[\mathrm{~V}_{2}(R)\right]-E_{\mathrm{tot}}^{N}\left[\mathrm{MoS}_{2}\right]-2 E_{\mathrm{V}}^{N}
$$

where $E_{\mathrm{tot}}^{N}\left[\mathrm{~V}_{2}(R)\right]$ is the total energy of a supercell with two Mo atoms a distance $R$ apart substituted with $\mathrm{V}$ atoms and the last two terms on the right do not depend on $R$. We consider the two cases where relaxation is (Re) and is not (Un) included.

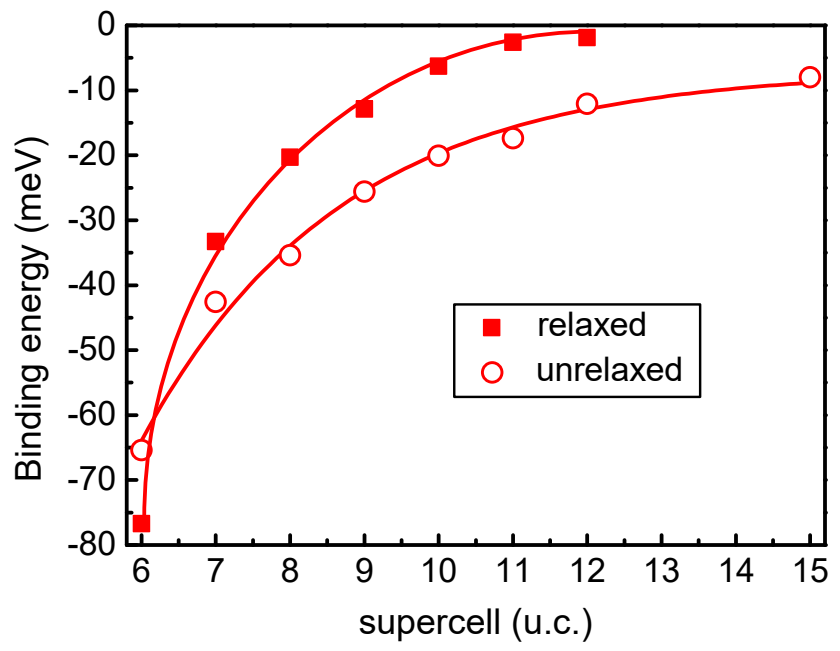

Figure 3.11: $E_{b}^{N}\left(R_{\max }\right)$ as a function of the supercell size $N$ for spin-polarized unrelaxed (open red circles) and relaxed (filled red squares) geometries. The red lines are a guide for the eye.

For supercells containing two substitutional $\mathrm{V}$ atoms as far apart as possible $\left(R_{\max }\right)$, with one $\mathrm{V}$ atom at the origin and the second at the corner site in Fig. 3.3, the binding energy $E_{b}^{N}\left(R_{\max }\right)$ is shown as a function of the supercell size $N$ in Fig. 3.11 with spin polarization included. Although $E_{b}^{N}\left(R_{\max }\right)$ does not change 
much for $N \geq 12$, there is still a surprisingly large binding energy of $\sim 8 \mathrm{meV}$ for $N=15$ in the unrelaxed case. We can trace this to the near degeneracy of the minority-spin $e^{\prime}$ and $a_{1}^{\prime}$ related bands shown in Fig. 3.10(c) for the unrelaxed $\mathrm{V}_{\mathrm{Mo}}$ as well as the relatively long range of the $e^{\prime}$ holes. When relaxation is included, the hole becomes localized in the dispersionless minority-spin $a_{1}^{\prime}$ band, Fig. 3.10(e), it becomes much easier to converge the total energy (with respect to BZ sampling and self-consistency) and $E_{b}^{12}\left(R_{\max }\right)$ decreases fast to $\sim 2 \mathrm{meV}$ for $N=12$. In general, when there is a gap between occupied and unoccupied states, total energies can be converged better. Since the problem has to do with the (separation independent) reference energy $E_{\mathrm{V}}^{N}$, it turns out to be better to consider

$$
E_{b}^{N}(R)=E_{\mathrm{tot}}^{N}\left[\mathrm{~V}_{2}(R)\right]-E_{\mathrm{tot}}^{N}\left[\mathrm{~V}_{2}(R=\infty)\right]
$$

and approximate $E_{\text {tot }}^{N}\left[\mathrm{~V}_{2}(R=\infty)\right] \sim E_{\text {tot }}^{N}\left[\mathrm{~V}_{2}\left(R_{\max }\right)\right]$

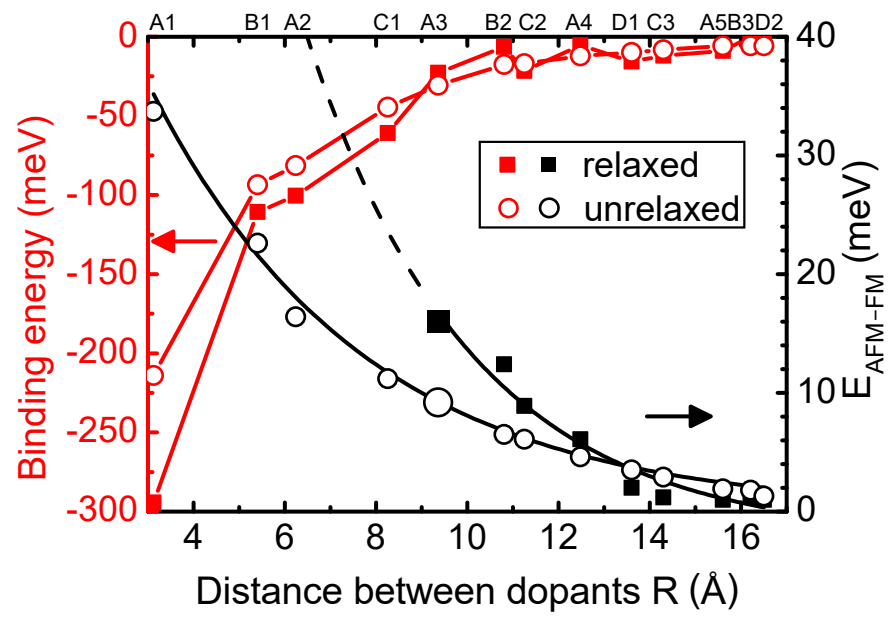

Figure 3.12: Red symbols: interaction energies of dopant $\mathrm{V}_{\mathrm{Mo}}$ atoms in a $12 \times 12$ $\mathrm{MoS}_{2}$ supercell as a function of their separation. The binding energy was calculated using (3.8) for unrelaxed (open red circles) and relaxed (filled red squares) geometries. The lines are a guide to the eye. Black symbols: total energy differences between parallel and antiparallel aligned spins on the $\mathrm{V}$ dopant atoms without (open black circles) and with (filled black squares) relaxation. The lines are fits to an exponentially decaying function. The dashed black line extrapolates the relaxed exchange interaction to separations where it is found to be quenched. The labels along the top of the figure indicate the sites in Fig. 3.3 and the large symbols refer to the A3 configuration discussed in the text and Fig. 3.16.

Using a $12 \times 12$ supercell and (3.8) we explore the pair binding energy $E_{b}^{12}(R)$ for $\mathrm{V}_{\text {Mo }}$ dopants as a function of their separation $R$ in Fig. 3.12 where one dopant atom is assumed at the site marked 0 in Fig. 3.3. Without relaxation, the (absolute value 
of the) binding energy decreases monotonically from a value of $\sim 220 \mathrm{meV}$ for $\mathrm{V}$ atoms on neighbouring Mo sites to a value of $\sim 0 \mathrm{meV}$ at the maximum separation in a $12 \times 12$ supercell (open red circles, left axis). Because these energies are so small, we will later assume that dopant atoms are randomly distributed in real materials that are not in full thermodynamic equilibrium.

With relaxation (filled symbols), the magnitude of the binding energy increases for separations $R$ smaller than a critical separation, $R_{c} \sim 8.5 \AA$, and does not change for separations larger than this. This behaviour is intimately related to quenching of the magnetic moments for $\mathrm{V}$ dopant atoms closer than $R_{c}$ and for these separations, $R<R_{c}$, an exchange interaction cannot be determined. We proceed to consider the magnetic interactions.

\subsubsection{Magnetic Interaction of impurity pairs}

We estimate the exchange interaction $\Delta E(R)$ between pairs of dopant atoms as the energy difference between configurations with the $\mathrm{V}$ magnetic moments aligned parallel ("ferromagnetically", FM) and antiparallel ("antiferromagnetically", AFM)

$$
\Delta E(R)=E_{\text {tot }}\left[\mathrm{V}_{2}^{\mathrm{AFM}}(R)\right]-E_{\text {tot }}\left[\mathrm{V}_{2}^{\mathrm{FM}}(R)\right]
$$

in $12 \times 12$ supercells so that the interaction between periodic images is acceptably small. Because the spin-polarized calculations are computationally expensive, care is taken to construct suitable starting $\mathrm{V}_{2}^{\mathrm{FM}}$ configurations using "superpositions" of

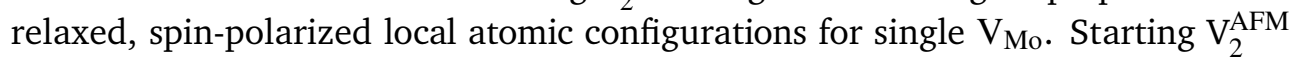
configurations are constructed from relaxed $\mathrm{V}_{2}^{\mathrm{FM}}$ configurations so only the much smaller differential relaxation needs to be calculated.

The energy difference between antiferromagnetic and ferromagnetic ordering without (open black circles) and with (filled black squares) relaxation is shown on the right axis of Fig. 3.12. Before relaxation, neighbouring $\mathrm{V}$ dopant atoms show FM coupling with a total moment of $2 \mu_{B}$ or $1 \mu_{B}$ per $V$ for all separations. The interaction decreases monotonically and exponentially from a maximum of $\sim 33 \mathrm{meV}$ for nearest neighbours with a decay length of $\sim 5.3 \AA$. With relaxation included the magnetic moments are quenched for separations $R$ smaller than a critical separation, $R_{c} \sim 8.5 \AA$, and for these separations an exchange interaction cannot be determined. For separations $R>R_{c}$, the coupling remains ferromagnetic and is enhanced. Because the maximum value of the magnetic ordering temperature will depend strongly on this relaxation-induced behaviour, we need to understand its origin.

\section{Quenching of moments for $R<R_{c}$}

To do so, we consider a $12 \times 12$ supercell for an $\mathrm{MoS}_{2}$ monolayer with a pair of Mo atoms on neighbouring sites substituted with V. The unpolarized supercell elec- 

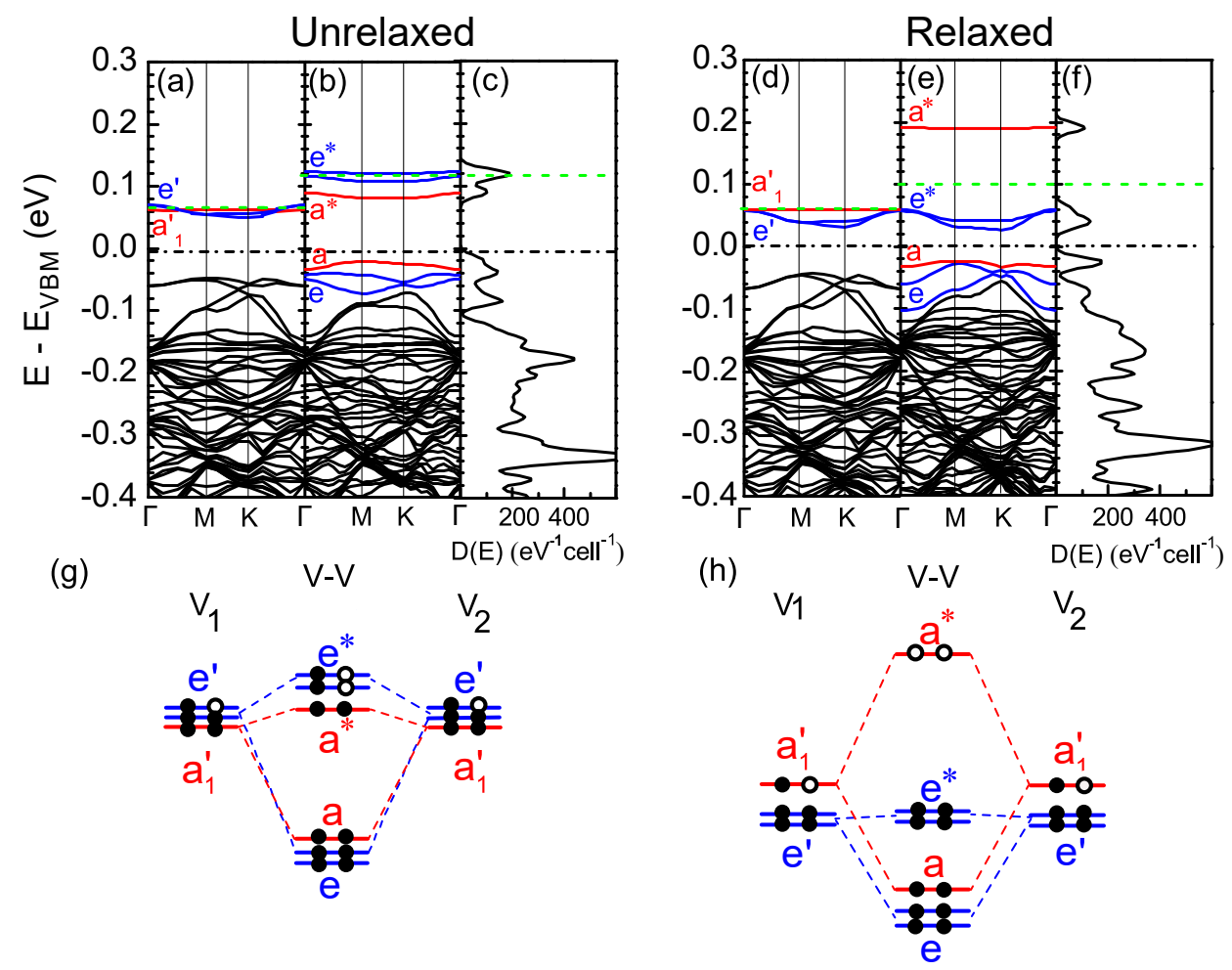

Figure 3.13: Spin unpolarized electronic structure (bands and DoS) for a $12 \times 12$ $\mathrm{MoS}_{2}$ supercell with two $\mathrm{V}$ atoms in an A1 configuration on nearest neighbour Mo sites (b,c,e,f) and, for comparision, for a single $\mathrm{V}_{\mathrm{Mo}}$ atom (a,d) corresponding to Fig. 3.10((b,d). Without (a,b,c,g) and with (d,e,f,h) atomic relaxation. Schematic of the coupling mechanism between two $\mathrm{V}$ dopants with two holes without $(\mathrm{g})$ and with (h) atomic relaxation. Impurity bands are highlighted in red and blue. The $\mathrm{K}$ point VBM is set to be zero (dot-dashed black line) and the Fermi level is shown as a dashed green line.

tronic structures and DoS are shown in Fig. 3.13 without (left) and with relaxation (right). The corresponding band structures for a single $\mathrm{V}$ impurity are included in the left panels for reference. In the spirit of a defect molecule model, Fig. 3.13(a-c) suggests that the $e^{\prime}$ orbitals form $\sigma$ bonding-antibonding $e-e^{*}$ pairs scarcely lifting the degeneracy of the $e$ states while the $a_{1}^{\prime}$ states interact less strongly to form a $\pi$ bonding-antibonding $a-a^{*}$ pair. Without relaxation, the strength of the $\pi$ bond between the $a_{1}^{\prime}$ orbitals is not strong enough to raise the $a^{*}$ level above the $e^{*}$ level and the two holes reside on the fourfold orbitally and spin degenerate $e^{*}$ states as sketched in Fig. 3.13(g). This leads to a DoS peak at the Fermi level that is unstable with respect to exchange splitting. 
(a) unrelaxed structure: $\pi$ bond

(b) unrelaxed structure: $\sigma$ bond
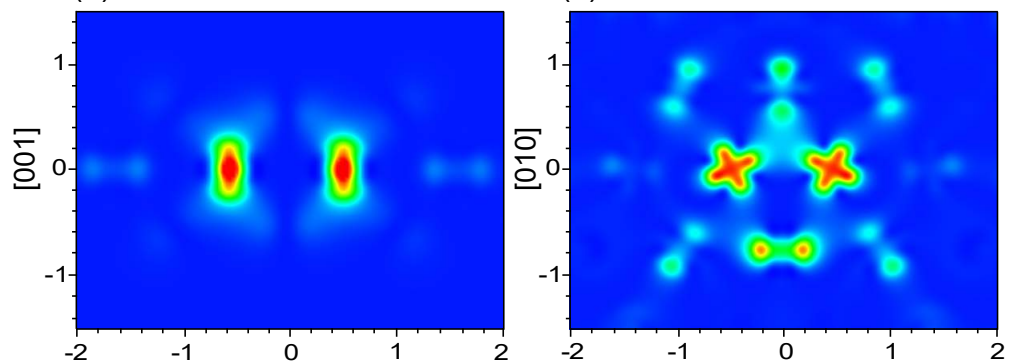

(c) relaxed structure: $\pi$ bond

(d) relaxed structure: $\sigma$ bond
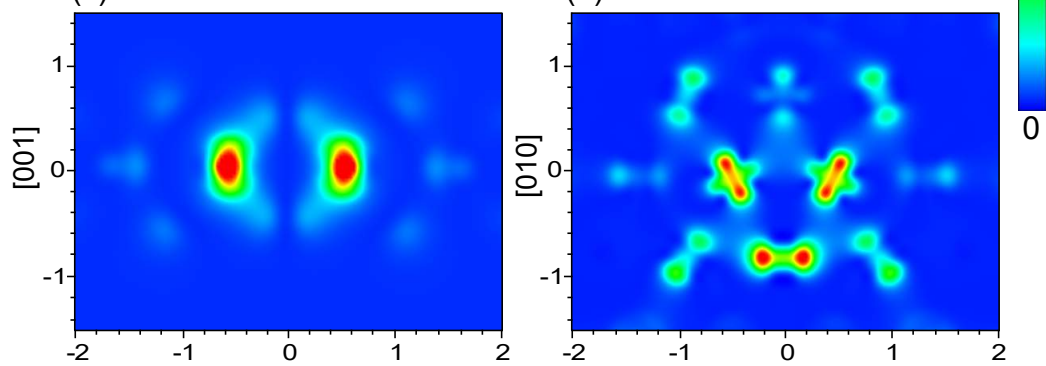

(e) atomic structure: $\pi$ bond

(f) atomic structure: $\sigma$ bond
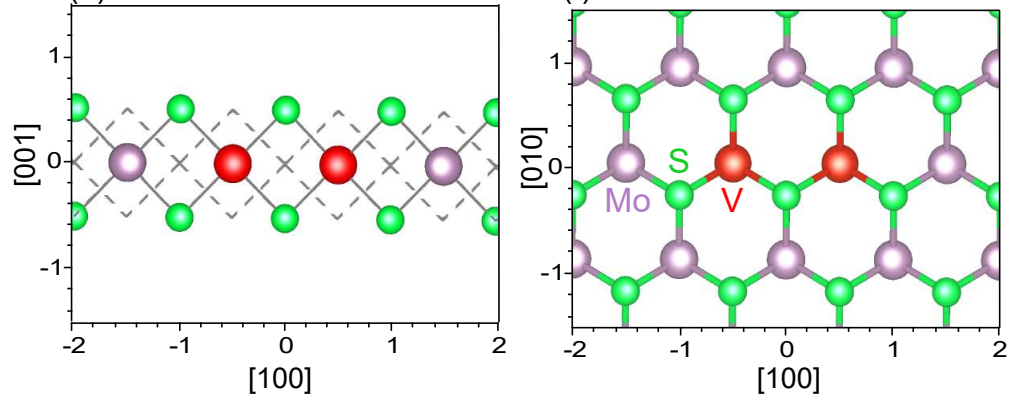

Figure 3.14: Side view of the partial charge distributions of the $\pi$ bond (left panels) and top view of the $\sigma$ bond (right panels) without (a, b) and with (c, d) atomic relaxation. The corresponding atomic structures are shown in $(e, f)$.

Relaxation results in a structure where the neighbouring $S$ atoms move closer to the $\mathrm{V}$ atoms, the two $\mathrm{V}$ atoms move apart and the $a_{1}^{\prime}$ levels on individual $\mathrm{V}_{\mathrm{Mo}}$ atoms are lifted clear of the $e^{\prime}$ levels. The reduced V-S separation strengthens the $\pi$ bond [Fig. 3.14(a) versus Fig. 3.14(c)] through hybridization between vanadium $d_{3 z^{2}-r^{2}}$ and sulphur $p_{x}$ and $p_{y}$ orbitals, while the $\sigma$ bonds formed by vanadium $\left\{d_{x y}, d_{x^{2}-y^{2}}\right\}$ orbitals are weakened by the increased V-V separation [Fig. 3.14(b) versus Fig. 3.14(d)]. This makes the $\pi$ bond the dominant bonding interaction between dopants. The $a-a^{*}$ splitting is increased so much by relaxation that the $a^{*}$ level is lifted well above the $e^{*}$ level and the two holes are accommodated in an orbitally nondegenerate state (rhs of Fig. 3.13). 
To demonstrate how competition between bonding and exchange interactions of the $\mathrm{V} d_{3 z^{2}-r^{2}}$ orbitals leads to the quenching of the magnetic moments, we examine how these interactions depend on the separation between the dopant atoms. We define the bond strength $\Delta_{\pi}$ of the $\pi$ bond to be the $a^{*}-a$ bonding-antibonding splitting. Fig. 3.15 shows how $\Delta_{\pi}$ depends on the impurity separation $R$ with (filled black squares) and without (open black circles) structural relaxation. As $R$ increases, $\Delta_{\pi}$ decreases because of the decreasing wavefunction overlap. Without relaxation (open circles), $\Delta_{\pi}$ is smaller than the exchange splitting (red triangles, dashed red line) for all separations and a triplet state would form as indicated in the rhs inset of Fig. 3.15. With relaxation (filled squares), $\Delta_{\pi}$ is larger and exceeds the exchange splitting at distances smaller than $\sim 7 \AA$. A singlet state is formed to gain bonding energy, as sketched in the lhs inset of Fig. 3.15, and this leads to the quenching of the magnetic moment. The critical quenching separation is twice the effective Bohr radius $a_{0}^{*}=4.2 \AA$ for the $a_{1}^{\prime}$ state, implying the formation of a $\pi$ bond. In general, to quench the magnetic moment, the $\pi$ bonding interaction should be strong enough to make the $a^{*}$ state the highest lying state.

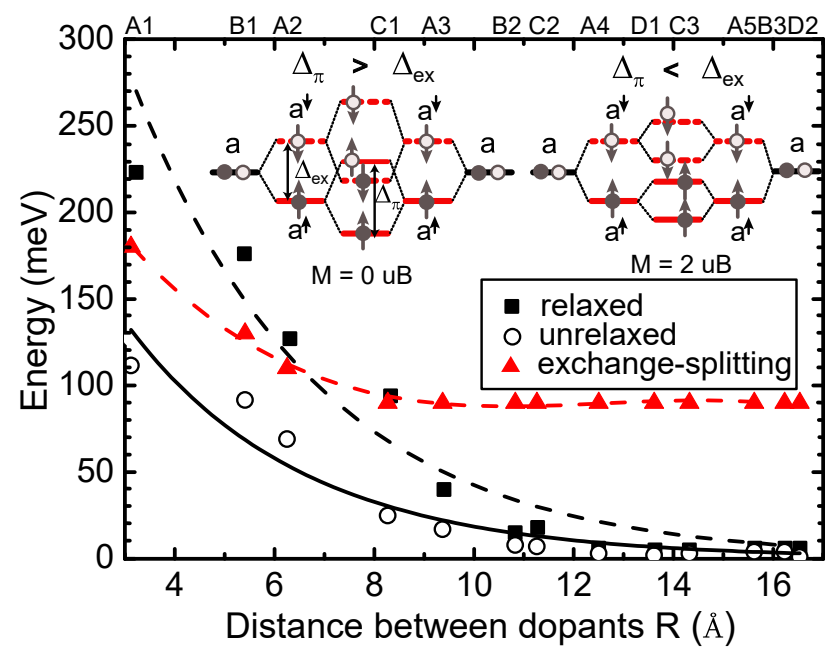

Figure 3.15: Unpolarized $a-a^{*}$ level separation with (filled black squares) and without (open black circles) atomic relaxation plotted as a function of the separation between the substitutional dopant atoms in a $12 \times 12 \mathrm{MoS}_{2}$ supercell. The $a^{*}$ exchange splitting for different (unrelaxed) configurations is indicated by red triangles. The insets show the energy level schemes with spin polarization included for relaxed configurations with impurity separations below (lhs) and above (rhs) the critical separation of $8.5 \AA$, respectively.

Lastly, we note a significant enhancement of the exchange splitting when two vanadium atoms are close, Fig. 3.15. From a value of $92 \mathrm{meV}$ for single V dopants, the increase in hole density at short separations doubles the exchange splitting to 
$\sim 180 \mathrm{meV}$ for (unrelaxed) V dopants on neighbouring Mo sites.

\section{Enhancement of Exchange Interaction for $R>R_{C}$}

For separations greater than $R_{c}$, the exchange interaction is strongly enhanced by relaxation before decaying more strongly than the unrelaxed case till it eventually becomes smaller when $R \sim 13 \AA$, Fig. 3.12. We can understand the enhancement by considering in Fig. 3.16 the defect levels associated with the A3 configuration, Fig. 3.3. For the unrelaxed structure (lhs), the breaking of the local $D_{3 h}$ symmetry is negligible and the bonding $e$ and antibonding $e^{*}$ states remain doubly degenerate. At this separation of $9.55 \AA$, the bonding interaction of the $a_{1}^{\prime}$ states is much less that of the $e^{\prime}$ states so that the $e^{*}$ level is the lowest unoccupied level to which both holes gravitate. Because it is degenerate, the $e^{*}$ level can exchange split with both holes aligned to form a triplet spin state. The exchange splitting is weak because of the delocalisation of the $e$ levels.

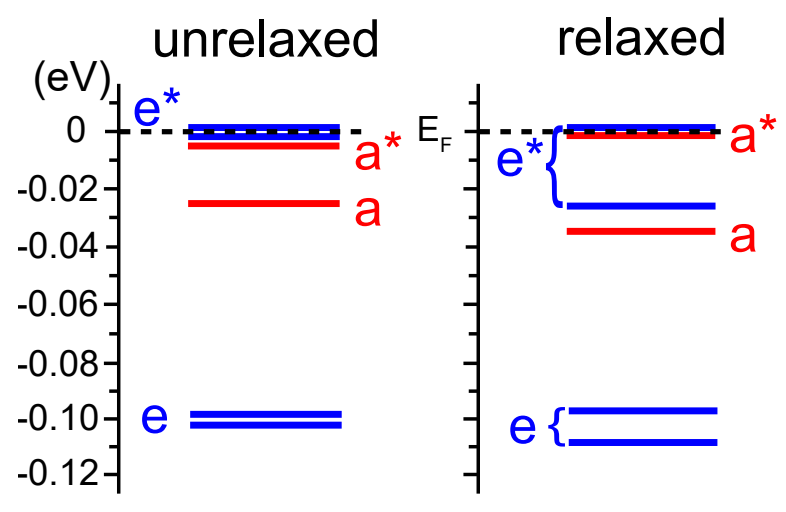

Figure 3.16: Defect level structure calculated without spin polarisation for an A3 configuration of two $\mathrm{V}_{\mathrm{Mo}}$ atoms. (lhs) unrelaxed and (rhs) relaxed. The levels are calculated from the appropriate weighted average of the $\Gamma, \mathrm{K} / \mathrm{K}^{\prime}$ and $\mathrm{M}$ eigenvalues of the corresponding bands. $a$ and $a^{*}$ (red), $e$ and $e^{*}$ (blue) levels originate in the $a_{1}^{\prime}$ and $e^{\prime}$ levels for single $\mathrm{V}_{\mathrm{Mo}}$ dopants. The zero of energy is the Fermi level indicated by a black dashed horizontal line.

Relaxation reduces the V-S bond length while increasing the V-V bond length and breaks the local $D_{3 h}$ symmetry leading to a significant splitting of the degenerate $e$ and $e^{*}$ levels as well as a small increase in the bonding interaction between the $a_{1}^{\prime}$ levels (Fig. 3.16, rhs). The net result is that the $a^{*}$ level and highest $e^{*}$ level become degenerate and accommodate the two holes. Because of the greater localization of the $a$ levels, this leads to an enhancement of the exchange splitting for the parallel (FM) configuration of the two $\mathrm{V}_{\mathrm{Mo}}$ dopants (compared to the unrelaxed case) and a reduction for the antiparallel (AFM) configuration with a correspond- 
ing increase of the $E_{\mathrm{AFM}}-E_{\mathrm{FM}}$ energy difference (large symbols in Fig. 3.12). To a good approximation the interaction strength only depends on the separation and decays exponentially more rapidly than the unrelaxed case with a much reduced decay length of $3.6 \AA$ reflecting the greater localization of the $a_{1}^{\prime}$ holes.

\subsubsection{Nb and Ta in $\mathrm{MoS}_{2}$}

We expect $\mathrm{Nb}$ and $\mathrm{Ta}$ to more closely resemble Mo than $\mathrm{V}$ with a weaker central cell potential leading to less localized impurity states than in the case of $\mathrm{V}$. Nb and Ta will turn out to have very similar effective Bohr radii and binding energies that lead to virtually indistinguishable magnetic properties.

\section{Single impurity limit: $\mathrm{Nb}$ and Ta in $\mathrm{MoS}_{2}$}

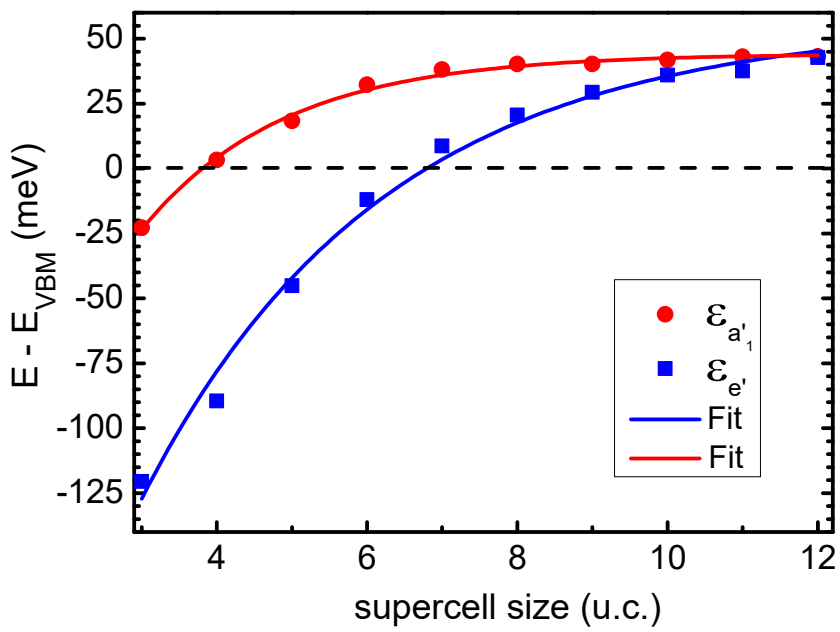

Figure 3.17: Same as Fig. 3.7 but for $\mathrm{Nb}$ instead of $\mathrm{V}$. The corresponding results for Ta are virtually indistinguishable.

For an unrelaxed $\mathrm{Nb}(\mathrm{Ta})$ substitutional impurity, we find an effective Bohr radius of 10.0 (10.3) $\AA$ for the $e^{\prime}$ state and 5.3 (5.2) $\AA$ for the $a_{1}^{\prime}$ state by fitting the circularly averaged wave function in a $12 \times 12$ supercell to be compared to values of $8 \AA$ and $4.2 \AA$, respectively, for $\mathrm{V}$ found in Sec. 3.3.1, Table 3.2. The binding energies of these $e^{\prime}$ and $a_{1}^{\prime}$ states converge to a common value of $\sim 45 \mathrm{meV}$ in the large supercell, single impurity limit Fig. 3.17; the results for $\mathrm{Nb}$ and $\mathrm{Ta}$ are virtually indistinguishable and only those for Nb are shown. The smaller binding energies and larger effective Bohr radii make the $\mathrm{Nb}(\mathrm{Ta})$ impurity states more sensitive to the supercell truncation of the impurity potential compared to V. Increasing delocalization of the holes from $\mathrm{V} \rightarrow$ Ta leads to a reduction of the exchange splittings, 
Table 3.6, and a magnetic moment is found to develop only when $N>5$. Total polarization only occurs for $N>10$, see Table 3.4.

After relaxation, the Nb-Mo (Ta-Mo) bond length increases by 0.040 (0.036) $\AA$, leading to a lowering of the $e^{\prime}$ state. The hole then goes into the $a_{1}^{\prime}$ state whose exchange splitting increases while that of the $e^{\prime}$ state decreases because of the reduced overlap in space of the $e^{\prime}$ and $a_{1}^{\prime}$ partial electron densities. As we already saw for $\mathrm{V}$ in Table 3.4, relaxation enhances the magnetic moments.

Table 3.6: Summary of the exchange splitting $\Delta_{\mathrm{ex}}$ in meV of $a_{1}^{\prime}$ and $e^{\prime}$ bound states in unrelaxed (Un) and relaxed (Re) structures in $12 \times 12$ supercells.

\begin{tabular}{lcccccccc}
\hline & \multicolumn{3}{c}{$\mathrm{V}$} & & \multicolumn{2}{c}{$\mathrm{Nb}$} & & \multicolumn{2}{c}{$\mathrm{Ta}$} \\
\cline { 2 - 3 } \cline { 8 - 9 }$\Delta_{\mathrm{ex}}$ & $a_{1}^{\prime}(\Gamma)$ & $e^{\prime}(\mathrm{K})$ & & $a_{1}^{\prime}(\Gamma)$ & $e^{\prime}(\mathrm{K})$ & & $a_{1}^{\prime}(\Gamma)$ & $e^{\prime}(\mathrm{K})$ \\
\hline Un & 91.3 & 24.7 & & 32.4 & 10.9 & & 35.6 & 10.8 \\
$\operatorname{Re}$ & 96.1 & 15.2 & & 53.2 & 9.8 & & 52.0 & 9.7 \\
\hline
\end{tabular}

\section{Interaction of $\mathrm{Nb}(\mathrm{Ta})$ impurity pairs}

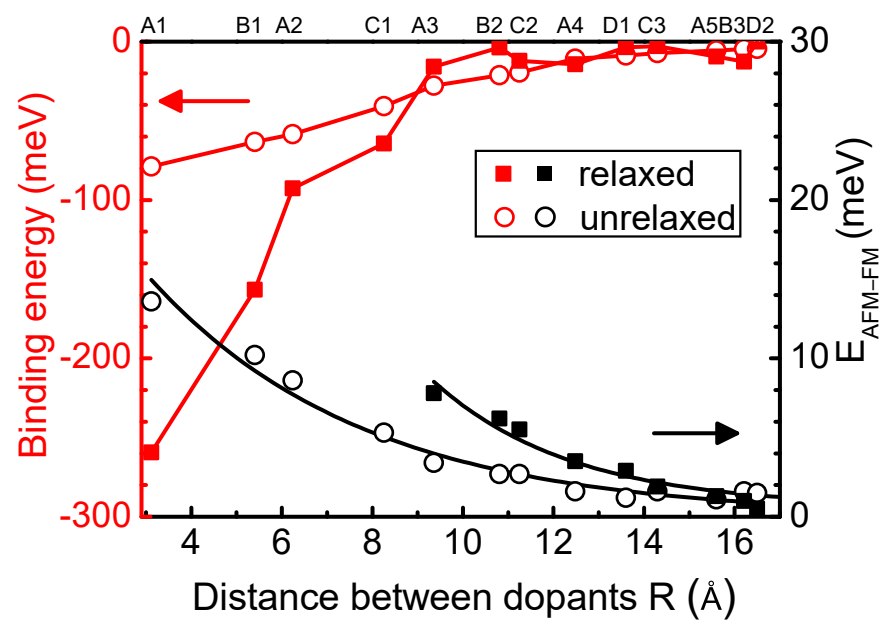

Figure 3.18: Same as Fig. 3.12 but for $\mathrm{Nb}$ instead of V. The corresponding results for Ta are virtually indistinguishable.

Because of the smaller bound state energies and weaker spin polarization, the exchange interaction between pairs of $\mathrm{Nb}(\mathrm{Ta})$ dopant atoms is weaker than that between pairs of V atoms, Fig. 3.12, and decays more slowly with increasing separation, Fig. 3.18. To a good approximation the interaction strength only depends on the separation $R$ and decays exponentially with $R$ with a decay length of 5.2 (5.8) $\AA$ versus 3.6 for $V$ when relaxation is included. 
The exchange splitting of the $a_{1}^{\prime}$ and $e^{\prime}$ levels and their relative hole occupations determine the strength of their different exchange interactions. Relaxation raises the $a^{*}$ level to become degenerate with the upper $e^{*}$ level, enhancing the exchange splitting of the $a$ level while reducing that of the $e$ level (see Table 3.6) and changes the character of the holes from $e$-like to $a+e$-like. This increases the strength of the exchange interaction but leads to a faster decay as we saw in Figs 3.12 and 3.18 for vanadium.

In summary, before relaxation, the long-range weak $e^{\prime}$ ferromagnetic interaction dominates while the strong short-range $a_{1}^{\prime}$ interaction dominates after relaxation, the near-degeneracy of the upper $e^{*}$ and $a^{*}$ levels making it possible to form a triplet without violating the Pauli exclusion principle.

\subsection{Magnetic Ordering}

The Ising spin model in two dimensions undergoes a phase transition to long-range magnetic order at a finite temperature [130; 131]. For a Heisenberg model with isotropic exchange interactions, thermal fluctuations destroy long-range magnetic ordering in two dimensions at any finite temperature [63; 64]. The Ising spin model, with spin dimensionality $n=1$, is recovered by assuming a generalized Heisenberg spin Hamiltonian with isotropic exchange and strong perpendicular anisotropy. Though the predictions of such generalized Heisenberg models are not identical to those of the Ising spin model, the consensus is that for ferromagnetism to exist in two-dimensional systems, magnetic anisotropy is essential. We therefore begin this section on magnetic ordering by studying the magnetic anisotropy of a single substitutional dopant, the so-called single ion anisotropy (SIA).

\subsubsection{Single ion anisotropy}

Microscopically, magnetic anisotropy arises when spin and orbital degrees of freedom are coupled by the spin-orbit interaction so that the total energy depends on the spatial orientation of the magnetic moment. According to the "force theorem" [132; 133], changes to the total energy, $\delta E$, that result from a small perturbation can be related to changes in the sum of the single-particle eigenstates of the KohnSham equations [16] of DFT, $\delta E \sim \delta \sum_{i}^{\text {occ }} \varepsilon_{i}$, which should not be iterated to self consistency. The force theorem has been applied to the calculation of the magnetic anisotropy energy (MAE) where the perturbation is the spin-orbit coupling (SOC) [134] and comparison with explicit total energy calculations yields essentially perfect agreement for Fe, Co and Ni [135]. The advantage of the force-theorem approach is that it allows the MAE to be directly related to (changes to) the electronic structure $[136 ; 137]$ which are shown for the relaxed configuration of a single $\mathrm{V}$ atom in a $6 \times 6$ supercell of $\mathrm{MoS}_{2}$ in Fig. 3.19. 


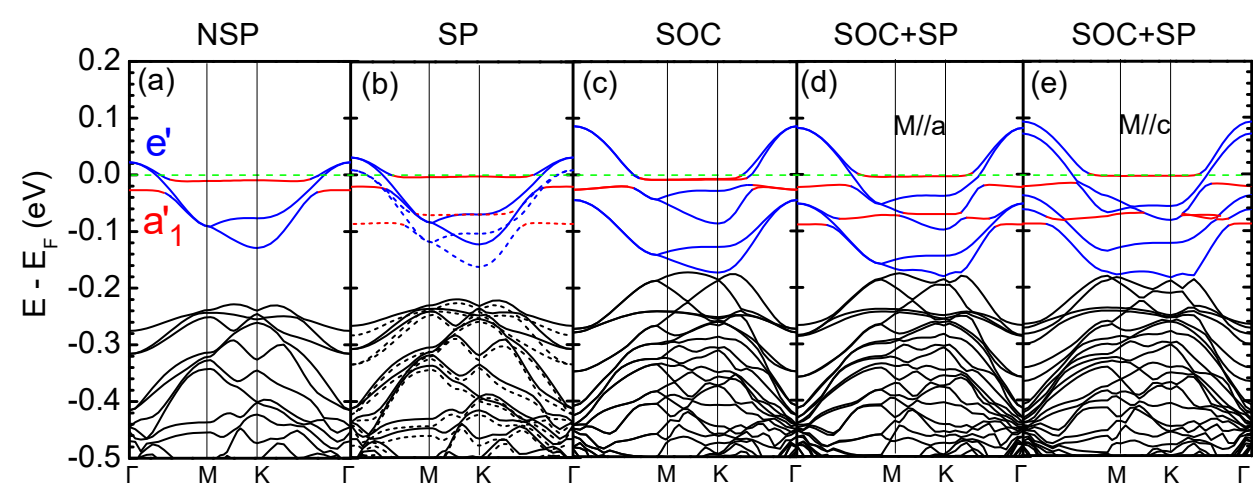

Figure 3.19: The band structure of a single $V$ dopant in a $6 \times 6$ supercell including relaxation. (a) non-spin polarized (NSP), (b) spin polarized (SP), (c) only spin orbit coupling (SOC). Including SOC and SP with the magnetization in-plane (M\|a) (d) and perpendicular to the monolayer plane $(\mathbf{M} \| c)$ (e). Because SOC does not mix the $e^{\prime}\left(m_{l}= \pm 2\right)$ and $a_{1}^{\prime}\left(m_{l}=0\right)$ states strongly, we can continue to label these blue and red. In (b), the solid and dashed lines represent spin-up and spin-down states, respectively. The Fermi level shown as a horizontal green dashed line is chosen to be zero.

In the context of Fig. 3.10(e), we already discussed the exchange splitting of the $a_{1}^{\prime}$ and $e^{\prime}$ levels. In a $6 \times 6$ supercell, the increased band dispersion leads to a smaller exchange splitting. With (without) relaxation, these splittings averaged over the Brillouin zone are, respectively, 66 (64) meV and 20 (22) meV (Fig. 3.19b). Because $\mathrm{MX}_{2}$ monolayers do not have inversion symmetry, SOC leads to a substantial splitting of the spin degenerate states at $\mathrm{K}$ and $\mathrm{K}^{\prime}$ with $\left\{d_{x y}, d_{x^{2}-y^{2}}\right\}$ character ( $l=2, m= \pm 2$ ) [138]. In Fig. 3.19(c), we see that SOC splits the $e^{\prime}$ level at $\Gamma$ by $130 \mathrm{meV}$ while the $a_{1}^{\prime}$ level with $d_{3 z^{2}-r^{2}}$ character $(l=2, m=0)$ is not affected. For a larger $(12 \times 12)$ supercell, the effect of SOC on the bands shown in Fig. 3.10(d) is to split the upper $e^{\prime}$ level so that it lies above the unaffected $a_{1}^{\prime}$ level and accommodates the hole.

To understand the energy levels obtained with SOC and spin polarization (exchange splitting) in the single impurity limit, it is instructive to consider the model Hamiltonian

$$
H=H_{0}+\Delta \mathbf{m} \cdot \mathbf{s}+\xi \mathbf{l} \cdot \mathbf{s}
$$

where $H_{0}$ is the spin-independent part of the Hamiltonian, $\Delta \mathbf{m}$ is the exchange field that leads to an exchange splitting $\Delta$, and $\mathbf{m}$ is a unit vector in the direction of the magnetization, $\mathbf{m} \equiv \mathbf{M} /|\mathbf{M}|$. In the subspace of the $l=2, m_{l}= \pm 2$ orbitals

$$
\xi \mathbf{l} \cdot \mathbf{s}=\frac{\xi}{2}\left(\begin{array}{cc}
l_{z} & l_{-} \\
l_{+} & -l_{z}
\end{array}\right)=\left(\begin{array}{cc}
\xi & 0 \\
0 & -\xi
\end{array}\right)
$$


where we use Hartree atomic units with $\hbar=1$. For $\mathbf{M} \| c$,

$$
\Delta \mathbf{m} \cdot \mathbf{s}=\left(\begin{array}{cc}
\frac{\Delta}{2} & 0 \\
0 & -\frac{\Delta}{2}
\end{array}\right) .
$$

and the SOC Hamiltonian can be written as

$$
H=H_{0}+\left(\begin{array}{cccc}
\xi+\frac{\Delta}{2} & 0 & 0 & 0 \\
0 & -\xi-\frac{\Delta}{2} & 0 & 0 \\
0 & 0 & -\xi+\frac{\Delta}{2} & 0 \\
0 & 0 & 0 & \xi-\frac{\Delta}{2}
\end{array}\right)
$$

For $\mathbf{M} \| a$ we have

$$
H=H_{0}+\left(\begin{array}{cccc}
\xi & \frac{\Delta}{2} & 0 & 0 \\
\frac{\Delta}{2} & -\xi & 0 & 0 \\
0 & 0 & -\xi & \frac{\Delta}{2} \\
0 & 0 & \frac{\Delta}{2} & \xi
\end{array}\right)
$$

Diagonalizing $H$ results in the energy level scheme sketched in Fig. 3.20. The magnetic anisotropy energy is $E_{\mathrm{MAE}}=E_{a}-E_{c}$, where $E_{a}$ and $E_{c}$ are the total energies when $\mathbf{M} \| a$ and $\mathbf{M} \| c$, respectively. Using the force theorem, the energy change on including SOC is given by the change in the sum of single-particle eigenvalues. The reference energy (without SOC) cancels when the difference is taken for the two magnetization directions and, for occupancy with a single hole, $E_{\mathrm{MAE}}$ can be estimated to be

$$
E_{\mathrm{MAE}} \quad=E_{a}-E_{c}=\xi+\frac{\Delta}{2}-\sqrt{\xi^{2}+\frac{\Delta^{2}}{4}}
$$

where we make use of the fact that the sum over all single particle eigenvalues is zero to express the sum over occupied states in terms of the sum over unoccupied states that is simply the energy of the hole. In this simple model, it is clear that for single acceptors the energy is lower when the magnetization is out of plane. In the limit that $\Delta \ll \xi, E_{\mathrm{MAE}} \sim \frac{\Delta}{2}\left(1-\frac{\Delta}{4 \xi}\right)$.

To determine the MAE using the vASP code $^{\dagger}$, we adopt a two step procedure. We first perform a well-converged self-consistent spin-polarized calculation for the minimum energy geometry without SOC. The output from that calculation is used as input to the second step where SOC is added and the Kohn-Sham equation is solved non self-consistently yielding a new eigenvalue spectrum, Fermi energy and wavefunctions from which a total energy can be determined; to use the force theorem, we will just make use of the eigenvalue-sum part of the total-energy output. When adding the SOC, an orientation for the exchange field (magnetization direction) needs to be chosen and this will yield an orientation dependent eigenvalue

\footnotetext{
†The vaSP guide, https://cms.mpi.univie.ac.at/vasp/vasp.pdf
} 


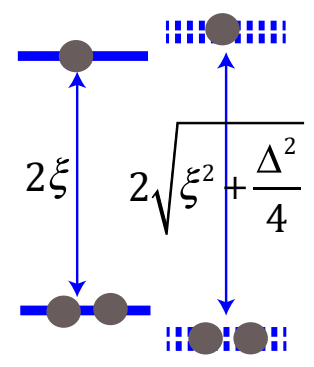

$\mathrm{M} / / \mathrm{a}$

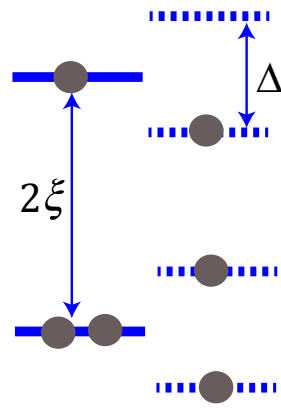

$\mathrm{M} / / \mathrm{c}$

Figure 3.20: Schematic of the $e^{\prime}$ energy levels with spin orientation in-plane ( $\left.\mathbf{M} \| a\right)$ and out of plane $(\mathbf{M} \| c)$. The solid blue lines are spin degenerate.

spectrum, Fermi energy etc. To determine the MAE, we need to perform two calculations with the magnetization chosen (i) perpendicular to the plane and (ii) in plane. The MAE will be expressed as the difference. To calculate the single particle eigenvalue sum for the electronic structure shown in Fig. 3.19 requires a careful BZ summation $[134 ; 139 ; 136]$ for which we use the improved tetrahedron method [140]. The results obtained using the force theorem for a $6 \times 6$ supercell and 4, 8 and 12 divisions of the reciprocal lattice vectors are shown in Fig. 3.21 as a function of the BZ area element $(2 s)$ normalized to the area, $S_{\mathrm{BZ}}$, of the BZ for a $1 \times 1$ primitive unit cell (black squares). An integral is defined as the limit where $s \rightarrow 0$ for an infinite number of sampling $\mathrm{k}$ points and from the figure we expect a value of $\sim 0.8 \pm 0.2 \mathrm{meV}$.

When the supercell size is increased and the dispersion of the $e^{\prime}$ and $a_{1}^{\prime}$ states becomes smaller, we might expect the BZ summation to converge faster but the situation is complicated by the near-degeneracy of the $e^{\prime}$ and $a_{1}^{\prime}$ bands. Fig. 3.21 includes results for $9 \times 9$ and $12 \times 12$ supercells indicating a strong increase in the size of the $E_{\mathrm{MAE}}$ in the single impurity limit. The strong dependence of the MAE on the supercell size can be understood in terms of the reduced dispersion of the impurity levels and the contribution to the MAE from states near the Fermi level whose degeneracy is lifted when the magnetization direction is rotated from inplane with $\mathbf{M} \| a$ to out-of-plane with $\mathbf{M} \| c$ as illustrated by Fig. 3.19(d,e) and Fig. 3.20. For a $12 \times 12$ supercell, we can extrapolate the results obtained using a $2 \times 2$ and $4 \times 4$ k-point sampling to estimate a converged MAE of $4.2 \mathrm{meV}$. The exchange-splitting $\Delta$ is $15.2 \mathrm{meV}$ for the $e^{\prime}$ level and using this value of $\Delta$ and $2 \xi=130 \mathrm{meV}$ in (3.15) yields a value of $E_{\mathrm{MAE}} \sim 7.2 \mathrm{meV}$ that is still larger than the $4.2 \mathrm{meV}$ estimate from the full calculation. We can extrapolate the results for the three sizes of supercell to $s=0$ and then plot the results as a function of the inverse supercell size (inset) to estimate the SIA in the infinite supercell limit to be 


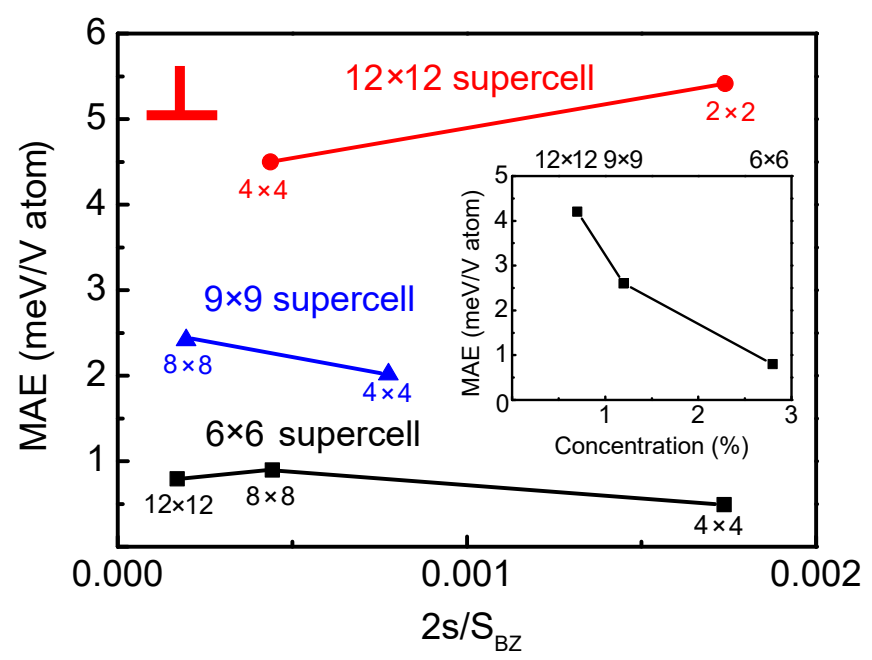

Figure 3.21: (color online). Convergence of the anisotropy energy of a relaxed substitutional $\mathrm{V}_{\mathrm{Mo}}$ atom in a monolayer of $\mathrm{MoS}_{2}$ for $6 \times 6$ (black), $9 \times 9$ (blue) and $12 \times 12$ (red) supercells as a function of the area, $s$, of the triangular surface element used to perform the two-dimensional BZ integral, given as a fraction of the total area of the $2 \mathrm{D} \mathrm{BZ}, S_{\mathrm{BZ}}$, for a $1 \times 1$ unit cell. The number of divisions of the reciprocal lattice vectors corresponding to each surface element is indicated for each supercell.

$4.5 \pm 0.5 \mathrm{meV}$ per $\mathrm{V}$ ion. This is much larger than the value reported for $2 \mathrm{D} \mathrm{CrI}_{3}$ [141] that exhibits Ising behavior [67]. The dipole-dipole interactions that play an important role in determining whether or not the magnetization of thin magnetic layers and magnetic multilayers is in-plane or out-of-plane are orders of magnitude smaller in the present case and can be safely neglected $[134 ; 139 ; 136]$.

\subsubsection{Monte Carlo calculations}

Very strong single-ion anisotropy combined with isotropic Heisenberg exchange results in Ising-like behaviour [142] which automatically gives a magnetically ordered phase at finite temperature $[130 ; 131]$. We map the energy differences calculated between FM and AFM oriented spins onto an isotropic Heisenberg exchange interaction and then model the V-doped $\mathrm{MoS}_{2}$ monolayer as an Ising spin system for which all odd moments disappear in zero field by symmetry. Monte Carlo calculations are used to determine the Curie temperature $T_{C}$ using Binder's cumulant method [143; 144] where the fourth order cumulant of the magnetization $\mathbf{M}$ simplifies to $U_{4}(T, L)=1-\left\langle M^{4}\right\rangle / 3\left\langle M^{2}\right\rangle^{2}$. As the system size $L \rightarrow \infty, U_{4} \rightarrow 0$ for $T>T_{C}$ and $U_{4} \rightarrow 2 / 3$ for $T<T_{C}$. For large enough lattice size, $U_{4}(T, L)$ curves for different values of $L$ cross as a function of temperature at a "fixed point" value 
$U^{*}$ and the location of the crossing fixed point is the critical point [144].

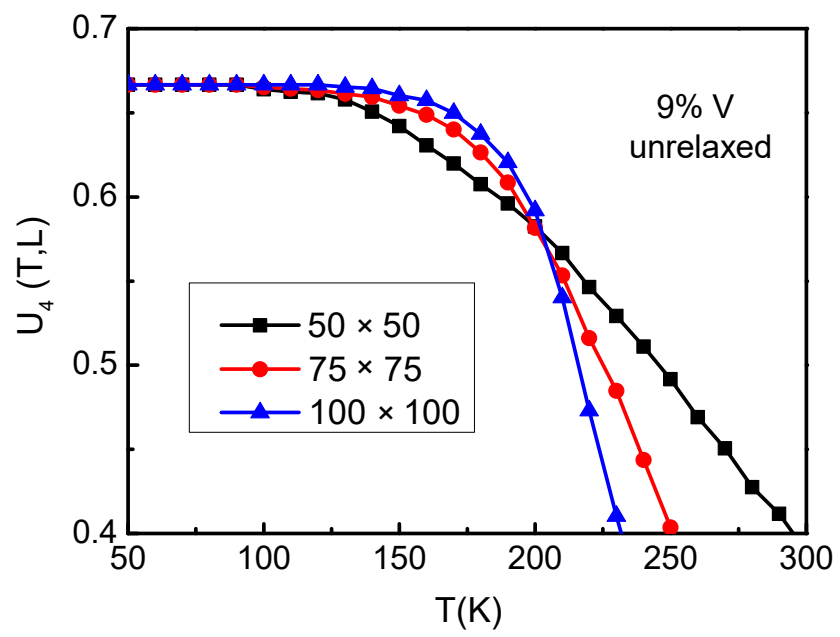

Figure 3.22: Variation of the fourth order cumulant for three different supercell sizes $L=50,75,100$ as a function of temperature.

At a given temperature and doping concentration, we establish thermodynamic equilibrium in $10^{5}$ Monte Carlo (MC) thermalization steps and then average over 48 different random dopant configurations to obtain $\left\langle M^{2}\right\rangle$ and $\left\langle M^{4}\right\rangle$. Three different lattice sizes with $L=50,75,100$ are used to calculate $U_{4}$ as a function of the temperature $T$ with doping concentrations from $1 \%$ to $11 \%$. An example of the results is shown in Fig. 3.22 where the fitting curve for the unrelaxed case in Fig. 3.12 is used to describe the exchange interactions for a doping concentration of $9 \%$. The temperature corresponding to the size independent universal fixed point $U^{*}$ where the $U_{4}(L, T)$ curves for different lattice sizes $L$ intersect yields an estimate for $T_{C}$. For the largest supercell size $L=100$, we calculated the magnetic susceptibility $\chi=\left[\left\langle M^{2}\right\rangle-\langle|M|\rangle^{2}\right] / N k_{b} T$ which diverges at the critical temperature in the thermodynamic limit [144]. An example is shown in Fig. 3.23 in which the Curie temperature obtained from the position of the magnetic susceptibility peak is in good agreement with that obtained from the fourth order cumulant.

\subsubsection{Curie temperature}

The ordering temperatures we calculate are shown in Fig. 3.24 for V doping concentrations $x$ in the range from $1 \%$ to $11 \%$. Without relaxation, $T_{C}(x)$ increases monotonically with doping concentration and reaches room temperature for a concentration of $\sim 11 \%$. With relaxation, the ferromagnetic exchange interaction is quenched for close dopant pairs and $T_{C}(x)$ exhibits a maximum of $\sim 165 \mathrm{~K}$ for $9 \%$ doping. If we extrapolate the exchange interaction for relaxed dopant pairs to sep- 


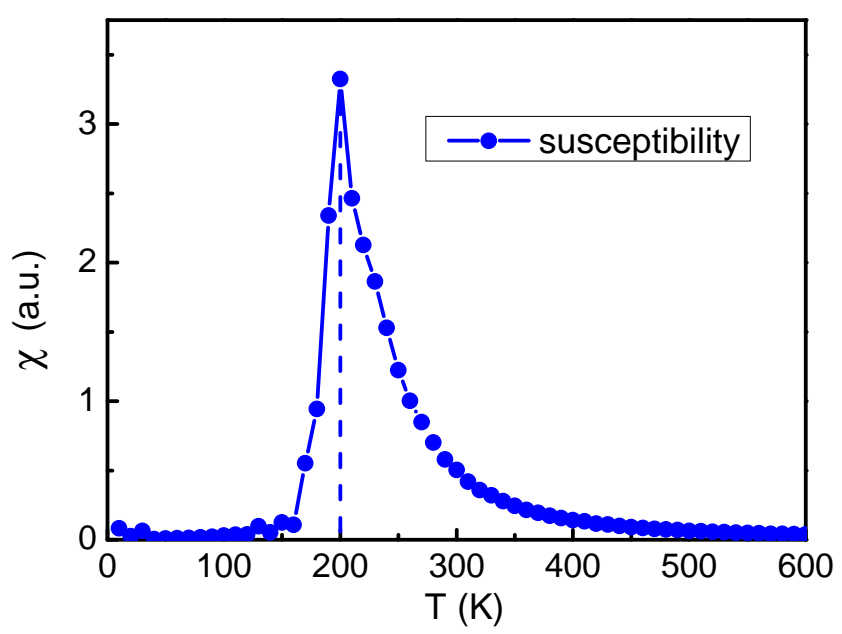

Figure 3.23: Variation of the magnetic susceptibility as a function of temperature for $9 \%$ unrelaxed V dopant concentration with $L=100$.

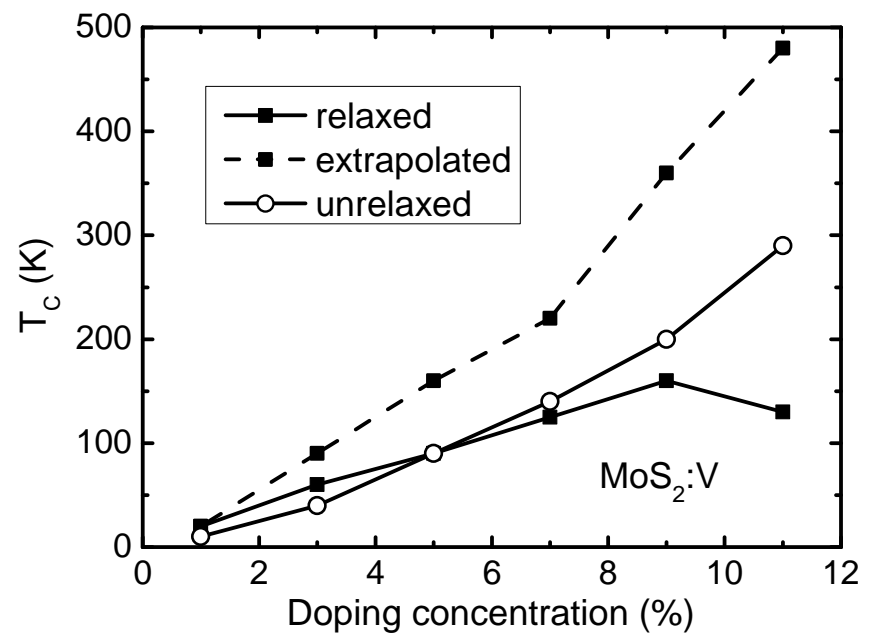

Figure 3.24: Variation of the Curie temperature as a function of the doping concentration calculated using Binder's cumulant method and the exchange interactions shown in Fig. 3.12 for an $\mathrm{MoS}_{2}$ monolayer doped with V. The dashed curve was calculated by extrapolating the exchange interaction for relaxed dopant pairs to separations shorter than the critical separations where quenching occurs. 
Table 3.7: Computational studies of single acceptor dopants in $\mathrm{MoS}_{2}$. SC: Supercell. XC: Exchange-Correlation functional. MAE: Magnetic Anisotropy Energy. US-PP: Ultrasoft pseudopotential [145]. NC-PP: Norm-conserving pseudopotential [146]. PAW: Projector Augmented-Wave [109]. LDA: Local Density Approximation [115]. CA: Ceperley-Alder [147]. GGA: Generalized Gradient Approximation. PBE: Perdew-Burke-Ernzerhof [116]. HSE: Heyd-Scuseria-Ernzerhof [148]. QE: QUANTUM ESPRESSO [149]. VASP: Vienna Ab-initio Simulation Package [111; 112]. WIEN2K [150]. SIESTA [151].

\begin{tabular}{|c|c|c|c|c|c|c|c|c|c|c|}
\hline \multicolumn{2}{|c|}{ SC size (\# atoms) } & \multirow[b]{2}{*}{ Dopant } & \multirow[b]{2}{*}{ Method } & \multirow[b]{2}{*}{$\mathrm{XC}$} & \multirow[b]{2}{*}{$\mathrm{U}(\mathrm{eV})$} & \multirow{2}{*}{$\begin{array}{l}\text { Vac. } \\
(\AA)\end{array}$} & \multirow[b]{2}{*}{ Code } & \multirow[b]{2}{*}{ MAE } & \multirow{2}{*}{$\begin{array}{l}\text { Exchange } \\
\text { Int. } J(d)\end{array}$} & \multirow[b]{2}{*}{ Reference } \\
\hline Default & Max/test & & & & & & & & & \\
\hline $4 \times 4$ & & $\mathrm{~V}, \mathrm{Nb}, \mathrm{Ta}$ & US-PP & GGA/PBE & 0 & 10 & $\mathrm{QE}$ & No & No & Cheng PRB13 [89] \\
\hline $4 \times 4$ & & $\mathrm{~V}$ & PAW & GGA/PBE & 0 & 12 & VASP & No & No & Yue PLA13 [90] \\
\hline \multirow[t]{2}{*}{$5 \times 5$} & $6 \times 6$ & $\mathrm{Nb}$ & NC-PP & LDA/CA & 0 & 15 & SIESTA & No & No & Dolui PRB13 [87] \\
\hline & & $\mathrm{Nb}$ & PAW & HSE & 0 & & VASP & No & No & \\
\hline $5 \times 5$ & & $\mathrm{~V}$ & PAW & GGA/PBE & 0 & 15 & VASP & No & No & Yun PCCP14 [93] \\
\hline $8 \times 8$ & & $\mathrm{~V}$ & PAW & GGA/PBE & 5.5 & & VASP & No & No & Andriotis PRB14 [96] \\
\hline $5 \times 5$ & $7 \times 7$ & $\mathrm{~V}, \mathrm{Nb}, \mathrm{Ta}$ & US-PP & GGA/PBE & 0 & 12 & $\mathrm{QE}$ & No & No & Lu NRL14 [82] \\
\hline $4 \times 4$ & & $\mathrm{~V}$ & PAW & GGA/PBE & 0 & 15 & VASP & No & No & Miao JMS16 [98] \\
\hline $5 \times 5$ & & V & PAW & GGA/PBE & 3 & 15 & VASP & No & Yes & Fan NRL16 [101] \\
\hline $6 \times 6$ & & $\mathrm{Cr}, \mathrm{V}$ & PAW & GGA/PBE & 0 & 20 & VASP & No & No & Robertson ACSN16 [102] \\
\hline \multirow[t]{2}{*}{$4 \times 4$} & $4 \times 4$ & $\mathrm{~V}, \mathrm{Cr}$ & FLAPW & GGA & 2.5 & 15 & WIEN2K & No & No & Singh AM17 [103] \\
\hline & $8 \times 5$ & $\mathrm{~V}$ & PAW & GGA/PBE & 0 & 16 & VASP & No & Yes & Miao ASS18 [104] \\
\hline $6 \times 6$ & & $\mathrm{Nb}, \mathrm{Ta}$ & PAW & HSE & 0 & 17 & VASP & No & No & Choi PRAP [152] \\
\hline $3 \times 3$ & $4 \times 4$ & $\mathrm{~V}$ & US-PP & GGA & 3 & 20 & $\mathrm{QE}$ & No & Yes & Mekonnen IJMPB18 [105] \\
\hline $12 \times 12$ & $15 \times 15$ & $\mathrm{~V}, \mathrm{Nb}, \mathrm{Ta}$ & PAW & LDA(GGA) & - & 20 & VASP & Yes & Yes & This work \\
\hline
\end{tabular}

arations smaller than the critical separation where quenching occurs (dashed line in Fig. 3.12), the Curie temperature increases rapidly and monotonically with doping concentration and exceeds room temperature for dopant concentrations larger than $9 \%$. Because the maximum value of $T_{C}(x)$ we obtain would be higher but for the quenching of the magnetic moments of closely separated relaxed dopants, it becomes important to consider how to suppress the quenching to obtain higher Curie temperatures. This will be discussed in Sec. 3.6.

\subsection{Comparison with other work}

Table 3.7 summarizes earlier computational work on doping $\mathrm{MoS}_{2}$ monolayers with $\mathrm{V}, \mathrm{Nb}$ or Ta. Because much of it was concerned with doping rather than with magnetic ordering, no attempts were made to calculate the magnetic anisotropy. Most of the calculations were done using small supercells and the separation dependence of the exchange interaction was not studied systematically, if at all. We noted in Sec. 3.2 that the GGA positions the $\Gamma$-point VBM too high with respect to the K-point VBM by comparison with experiment [114] and we therefore used the LDA that yields better agreement with experiment in this regard. Because most of the calculations referred to in Table 3.7 were performed with the GGA exchange-correlation potential, we examine the effect of using the GGA rather than the LDA. 


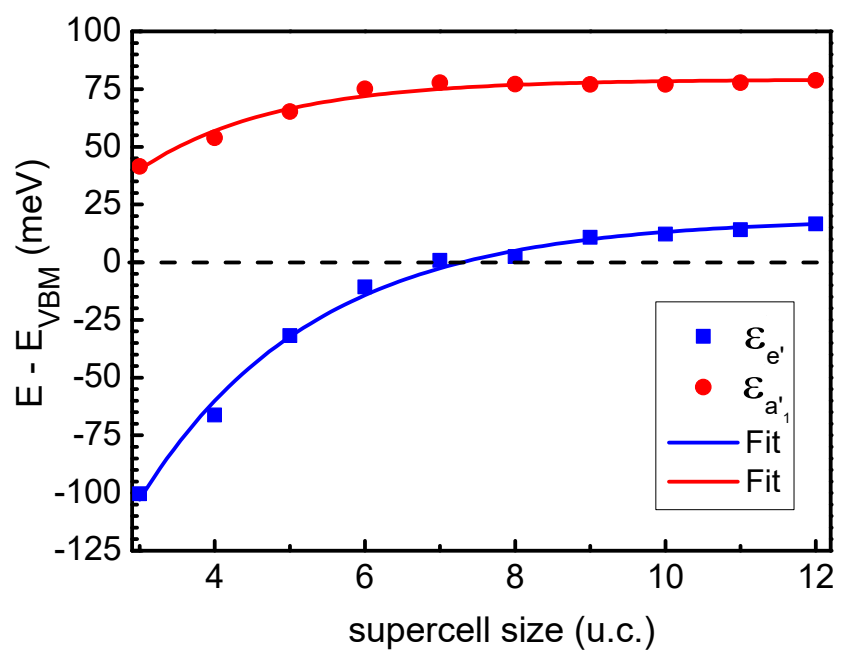

Figure 3.25: Dependence on the supercell size $N$ of the $a_{1}^{\prime}$ and $e^{\prime}$ impurity levels induced by an unrelaxed substitutional vanadium atom $V_{\text {Mo }}$ with respect to the valence band maximum in the GGA.

\section{GGA versus LDA}

If we use atomic configurations whose geometry was optimized using the LDA and repeat the electronic structure calculations using the GGA, we qualitatively reproduce the LDA results for the exchange interaction between $\mathrm{V}$ dopant atoms. The significant differences that will be documented below therefore have their origin in the only slightly different minimum-energy geometries predicted by the GGA.

Our starting point is an $\mathrm{MoS}_{2}$ monolayer whose lattice constant $a_{\mathrm{GGA}}=3.185 \AA$ minimizes the GGA total energy, Table 3.1. The GGA overestimate of the position of the $\Gamma$-point VBM with respect to the $\mathrm{K} / \mathrm{K}^{\prime} \mathrm{VBM}$ makes the $a_{1}^{\prime}$ level much higher than the $e^{\prime}$ levels as shown in Fig. 3.25 where these levels are plotted as a function of supercell size. The asymptotic values are $\sim 76$ and $\sim 20 \mathrm{meV}$ for the $a_{1}^{\prime}$ and $e^{\prime}$ states, respectively compared to $\sim 62 \mathrm{meV}$ for both in the LDA case, Fig. 3.7. Compared to the LDA estimate of a Bohr radius of $4.2 \AA$ from the radial extent of the partial charge density in Fig. 3.5, with the GGA we find a slightly larger value of $4.7 \AA$ for the $a_{1}^{\prime}$ state. By analogy with a hydrogen atom where the extent of the $1 s$ orbital increases greatly for the $\mathrm{H}^{-}$ion compared to the neutral atom, we attribute the slightly larger radial extent for the more strongly bound hole in the GGA case to its greater $a_{1}^{\prime}$ (hole) occupancy. In the GGA, the doped system is fully spin-polarized for the smallest $3 \times 3$ supercell we considered and the $a_{1}^{\prime}$ exchange splitting of $142 \mathrm{meV}$ is much larger than the $91 \mathrm{meV}$ we found in the unrelaxed LDA case. (The LDA predicts an exchange splitting of $\Delta \varepsilon_{1 s}=\varepsilon_{1 s}^{\uparrow}-\varepsilon_{1 s}^{\downarrow}=0.35$ Rydberg for a hydrogen atom compared to 0.55 Rydberg for the GGA; see Sec. 3.8.2). 


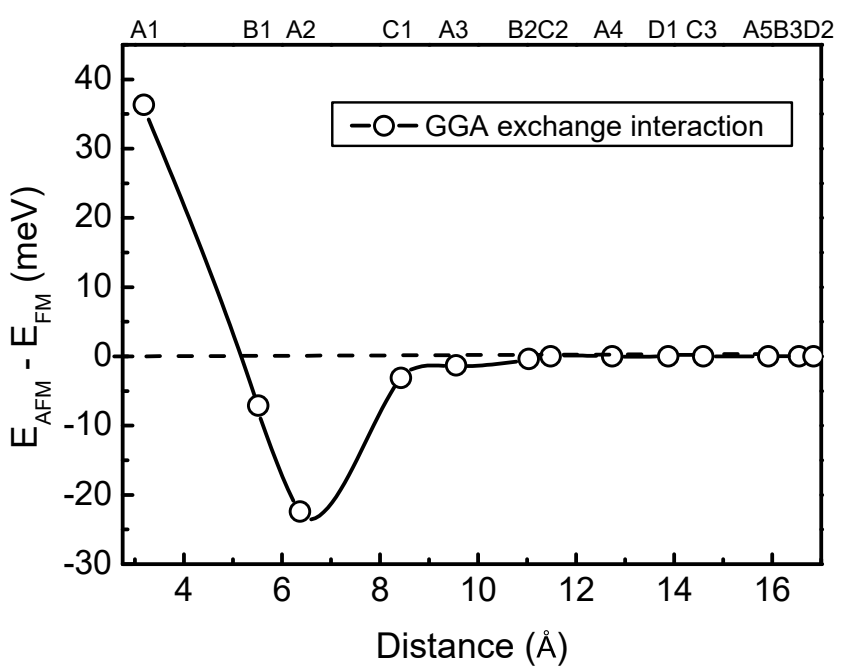

Figure 3.26: The total energy difference between parallel and antiparallel aligned spins on the V dopants without relaxation within GGA.

Fig. 3.26 shows the exchange interaction calculated with the GGA for pairs of unrelaxed $\mathrm{V}_{\mathrm{Mo}}$ dopants as a function of the distance between them. We observe an oscillatory behavior with FM coupling for neighbouring pairs ("A1" configuration, see Fig. 3.3) that switches to AFM for B1, A2, C1 and A3 configurations after which it is essentially zero reflecting the small effective Bohr radius of the $a_{1}^{\prime}$ level $\left(a_{0}^{*} \sim\right.$ $4.7 \AA$ ). The relatively large separation in energy of the $a_{1}^{\prime}$ and $e^{\prime}$ levels means that for separations larger than $\sim 9 \AA$, both holes are to be found in the anti-bonding $a^{*}$ level because the bonding-antibonding interaction is too weak to lead to hole occupancy of the $e^{*}$ level. Because both holes must of necessity occupy the $a^{*}$ level, their spins must be opposite and FM ordering is energetically unfavourable (though the energy difference is very small).

To understand the oscillation for small separations, we consider the electronic structures of the $a_{1}^{\prime}$ and $e^{\prime}$ derived $a-a^{*}$ and $e-e^{*}$ bonding and antibonding states for the A1 and A2 configurations in Fig. 3.27 with and without spin polarization. We begin with the A1 configuration. Compared to the LDA case that was shown in Fig. 3.13(b), we see that the unpolarized $a$ and $a^{*}$ levels (lhs of Fig. 3.27) are higher than the $e-e^{*}$ levels reflecting the corresponding feature for a single substitutional dopant and that the $a-a^{*}$ bonding-antibonding interaction is larger because the Bohr radius of the $a_{1}^{\prime}$ states is larger in the GGA. On the rhs of the A1 panel, we see that the exchange splitting of the $a^{*}$ levels is approximately doubled to $\sim 300 \mathrm{meV}$ for the $\mathrm{A} 1$ configuration compared to the single $\mathrm{V}_{\mathrm{Mo}}$ case because of the overlapping hole densities; the same doubling occurs in the LSDA, where the exchange splitting of $92 \mathrm{meV}$ for a single substitutional $\mathrm{V}$ is enhanced to $180 \mathrm{meV}$ for neighbouring 


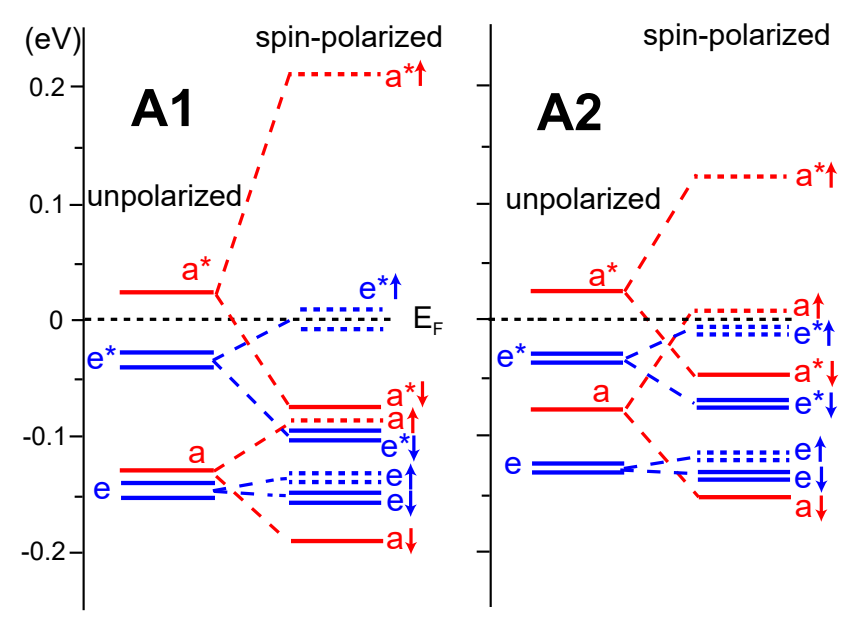

Figure 3.27: Energy diagrams for unrelaxed dopant pairs in A1 and A2 configurations (Fig. 3.3) with and without spin polarization calculated in GGA. The levels are calculated from the appropriate weighted average of the $\Gamma, \mathrm{K} / \mathrm{K}^{\prime}$ and $\mathrm{M}$ eigenvalues as in figures 3.13 and 3.16. The Fermi level is indicated as a black dashed horizontal line. The spin up and spin down states are represented as dashed and solid lines, respectively.

V pairs, see Fig. 3.15. The exchange splitting is so large that the down-spin $a^{*}$ level moves below the (approximately doubly) degenerate up-spin $e^{*}$ level with the result that the hole can flip its spin and the Fermi level is pinned in the half-filled up-spin $e^{*}$ state. By having one hole in an $a^{*}$ state and the other in an $e^{*}$ state it is possible for their spins to be parallel and to simultaneously gain spin-polarization and bonding energy.

As the separation between the $\mathrm{V}_{\mathrm{Mo}}$ atoms is increased, the exchange splitting and bonding interaction decrease rapidly for the $a$ states and a FM triplet state is only formed at the expense of having one hole occupying a bonding $a$ state (rhs of A2 panel); spin-polarization energy gain is offset by loss of bonding energy. Antiparallel alignment of the holes allowing gain of both spin-polarization and bonding energy becomes more favourable.

The effect of relaxation is to push the $a_{1}^{\prime}$ level up in energy and increase the $a-a^{*}$ bonding-antibonding splitting so that the unpolarized electronic structure resembles that of Fig. 3.13(e) with both holes in the $a^{*}$ level. For dopant separations less than $\sim 10 \AA$ this leads to quenching of the magnetism. For separations larger than this, the $a_{1}^{\prime}$ levels $\left(a_{0}^{*} \sim 4.7 \AA\right)$ interact only weakly with each other leading to very small energy differences $E_{\mathrm{AFM}}-E_{\mathrm{FM}}$ and negligible exchange interaction because the $e^{\prime}$ related levels are too low in energy to be occupied.

As anticipated at the beginning of this section, we can trace the large difference 
between the GGA and LDA descriptions of the exchange interaction between pairs of $\mathrm{V}_{\mathrm{Mo}}$ dopants to the $2 \%$ difference between the LDA and GGA lattice constants. If we use the experimental lattice parameters listed in Table 3.1, the differences between LDA and GGA disappear and we find that near-degenerate $e^{\prime}$ and $a_{1}^{\prime}$ impurity levels with binding energies of $56 \mathrm{meV}$ in a direct band gap of $1.78 \mathrm{eV}$ couple ferromagnetically for all separations. The different orbital character of the $e^{\prime}$ and $a_{1}^{\prime}$ levels means that they are very sensitive to the in-plane lattice constant $a$ and out-of-plane $d_{\mathrm{SS}}$, respectively, whose ratio determines their relative positions. From Table 3.1, we see that the LDA ratio of 1.001 is much closer to the experimental value of 0.996 than the GGA ratio of 1.018 and argue that the LDA provides a more reasonable description of the relative position of the impurity levels.

When SOC is taken into consideration, the energy difference $\Delta_{\mathrm{K} \Gamma}$ between the $\mathrm{K} / \mathrm{K}^{\prime}$ and $\Gamma$-point valence band maxima increases from 150 to $216 \mathrm{meV}$ for LDA and from 12 to $88 \mathrm{meV}$ for GGA, respectively, because of the large spin-orbit splitting at the $\mathrm{K} / \mathrm{K}^{\prime}$ point, Table 3.8. The experimental value, $140 \mathrm{meV}$, is just in between making it unclear what will actually happen. To resolve this issue, experiment should focus on determining the position of the $a_{1}^{\prime}$ level with respect to the top of the valence band in the single impurity limit.

Because the relative position of the impurity states determines the exchange interaction between dopant atoms, we have performed exploratory calculations to tune the relative positions of the $\mathrm{K} / \mathrm{K}^{\prime}$ and $\Gamma$ valence band maxima and consequently of the $a_{1}^{\prime}$ and $e^{\prime}$ levels with strain. For GGA, a $1 \%$ compressive strain $(\Delta a<0)$ is found to lower the $a_{1}^{\prime}$ levels to be degenerate with the $e^{\prime}$ levels and we find FM coupling for all separations. $2 \%$ tensile strain $(\Delta a>0)$ lifts the $a_{1}^{\prime}$ levels far above the $e^{\prime}$ levels stabilizing the magnetic moment of single impurities but favouring singlet formation of impurity pairs and AFM coupling. So while tensile strain reduces the formation energies of $\mathrm{Nb}_{\mathrm{Mo}}$ and $\mathrm{Ta}_{\mathrm{Mo}}$ and facilitates $p$ doping of $\mathrm{MoS}_{2}$ [152], it is detrimental for ferromagnetic ordering.

Table 3.8: Effect of SOC on the band gap $\Delta \varepsilon_{g}$ and valence band alignment for an $\mathrm{MoS}_{2}$ monolayer in the LDA and GGA. $\Delta_{\mathrm{K} \Gamma}=\varepsilon_{\mathrm{K}}-\varepsilon_{\Gamma}$ is the energy difference between the valence band maximum (VBM) at the $\mathrm{K}$ and $\Gamma$ points.

\begin{tabular}{lcclcc}
\hline & \multicolumn{2}{c}{ No SOC } & & \multicolumn{2}{c}{ Including SOC } \\
\cline { 2 - 3 } \cline { 5 - 6 } & $\Delta \varepsilon_{g}(\mathrm{eV})$ & $\Delta_{\mathrm{K} \Gamma}$ & & $\Delta \varepsilon_{g}(\mathrm{eV})$ & $\Delta_{\mathrm{K} \Gamma}$ \\
\hline GGA & 1.650 & 0.012 & & 1.586 & 0.088 \\
LDA & 1.860 & 0.150 & & 1.787 & 0.216 \\
Exp & & & & $1.900^{a}$ & $0.140^{b}$ \\
\hline
\end{tabular}

${ }^{a}$ Ref.11 ${ }^{b}$ Ref.114

If the exchange interactions are so sensitive to the lattice constant and the ratio of $a$ to $d_{\mathrm{SS}}$, it might be useful to consider tuning this ratio by modifying $\Delta_{\mathrm{K} \Gamma}$ either 
using strain or by alloying, $\mathrm{Mo}(\mathrm{S} / \mathrm{Se} / \mathrm{Te})_{2}$. In the $\mathrm{MoSe}_{2}, \mathrm{MoTe}_{2}$ and $\mathrm{WSe}_{2}$ systems, the $a_{1}^{\prime}$ level lies so much lower than the $e^{\prime}$ level that using GGA or LDA with their different lattice parameters has little effect; the coupling is dominated by the long range of the $e^{\prime}$ levels.

\section{LDA + U}

Two of the studies cited in Table 3.7 use a finite value of $U$ to better describe onsite Coulomb repulsion between electrons in localized $d$ orbitals [96; 103]. We find that LDA+U [128] with modest values of $U$ makes the local magnetic moment more localized and enhances it. The (more localized) $a_{1}^{\prime}$ level is more sensitive to $U$ than the $e^{\prime}$ level.

A small value of $U$ (less than $1 \mathrm{eV}$ ) increases the exchange splitting of the $a_{1}^{\prime}$ level and increases the FM exchange interaction which would yield a larger $T_{\mathrm{C}}$ compared to calculations without $U$. A larger value of $U$ (larger than $3 \mathrm{eV}$ ) causes the hole to become even more localized and fully polarized even at very high concentrations $(25 \%)$. As a consequence, the exchange interaction decays more rapidly and the separation below which quenching occurs decreases because the bonding interaction decays more rapidly. Compared to LDA calculations, the Curie temperature would be lower at low dopant concentration but enhanced at high concentration.

In our LDA calculations, we find that the shallow vanadium $3 d$ orbitals hybridize strongly with S-3p and Mo-4d orbitals delocalizing the holes. We expect the Coulomb $U$ in our system to be small and with a small $U$, the Curie temperature should be enhanced. Our LDA results should thus represent a lower bound on the exchange interaction and ordering temperature.

\subsection{Discussion}

According to the Zener $p-d$ model used to interpret magnetic coupling in $\mathrm{Ga}(\mathrm{Mn}) \mathrm{As}$ dilute magnetic semiconductors [60], holes in As $(p)$ bonding states mediate the exchange interaction between strong local $\left(d^{5}\right)$ magnetic moments on $\mathrm{Mn}^{2+}$ dopant ions. In the present case, the magnetic moments that we find come from unpaired $\left(d^{1}\right)$ spins in gap states that are only weakly bound by the Coulomb potential of the dopant ions, Fig. 3.2. The large Bohr radii we find for these states, Table 3.2, allows them to overlap to form narrow bands and suggests that on-site Coulomb interactions may play a minor role and a model of itinerant ferromagnetism may be more appropriate than the various localized models used to study the $\mathrm{Ga}(\mathrm{Mn}) \mathrm{As}$ and related systems $[61 ; 62]$. When the holes are in orbitally nondegenerate $a_{1}^{\prime}$ levels, AFM coupling is favoured to satisfy Pauli's exclusion principle; when they are in the degenerate $e^{\prime}$ levels, FM coupling is preferred to minimize the Coulomb interaction. For $\mathrm{MoS}_{2}$, the (accidental) near-degeneracy of the $a_{1}^{\prime}$ and $e^{\prime}$ hole states leads 
to them competing to determine the magnetic properties whereby the strength of the exchange interaction is related to the exchange splitting of the impurity band and will be affected by the band dispersion for high doping concentrations.

In the low doping limit, the impurity states have no dispersion and are fully polarized. As the impurity concentration is increased, the impurity levels overlap to form narrow bands that broaden and eventually overlap the narrow Mo band that forms the top of the valence band. As seen in Fig. 3.8, the impurity bandwidth increases exponentially with increasing doping concentration. For the $9 \%$ $\mathrm{V}$ dopant concentration for which we find $T_{\mathrm{C}}$ to be a maximum, the $e^{\prime}$ impurity bandwidth is $\sim 400 \mathrm{meV}$. The $a_{1}^{\prime}$ band is narrower, only about a third as wide. Both bandwidths exceed the $91 \mathrm{meV}$ exchange splitting we find for single $\mathrm{V}$ impurities in Fig. 3.10 that would imply partial quenching of the magnetic moments. For the ordered V dopants studied in Table 3.4, this quenching occurs as the concentration is increased above $3 \%$ and is complete by $6 \%$. For itinerant electrons occupying narrow bands, it has been argued that the effective interaction predicted by the Stoner criterion will not be reduced by correlation effects or spin wave excitations [108]. In contrast to traditional dilute magnetic semiconductors with large local moments that do not contribute to the spin stiffness, the completely spin polarized carriers in narrow impurity bands lead to a large spin stiffness and develop ferromagnetic ordering by their mutual interaction.

The quenching of ferromagnetic pairing for close impurity pairs can be avoided by considering instead of $\mathrm{MoS}_{2}$ as host semiconductor, $\mathrm{MoSe}_{2}$ or $\mathrm{MoTe}_{2}\left(\mathrm{WSe}_{2}\right.$ or $\mathrm{WTe}_{2}$ ) for which the $\Gamma$ point VBM drops with respect to the $\mathrm{K} / \mathrm{K}^{\prime} \mathrm{VBM}$ as $\mathrm{S} \rightarrow \mathrm{Se} \rightarrow \mathrm{Te}$ (sketched in Fig. 3.28). Preliminary calculations show that the $a_{1}^{\prime}$ defect levels do indeed follow the $\Gamma$ point VBM leaving the holes in the orbitally degenerate $e^{\prime}$ derived impurity bands. The increased lattice constant makes the $e^{\prime}$ states more localized and enhances the spin polarization in $\mathrm{MoSe}_{2}$ and $\mathrm{MoTe}_{2}$. Very recently there have been reports of long-range and/or room temperature ferromagnetism occurring in $\mathrm{V}$ doped $\mathrm{WSe}_{2}$ monolayers [153], in $\mathrm{MoSe}_{2}$ and $\mathrm{MoTe}_{2}$ [154], in V and Ta doped $\mathrm{MoTe}_{2}[155 ; 156]$ and in $\mathrm{MoS}_{2}$ [157] whereby the interaction with anion vacancies would appear to play an important role. These systems clearly warrant closer study.

We might expect double acceptors to have larger magnetic moments and exchange interactions. However, when $\mathrm{MoS}_{2}$ is doped with Ti, Zr, Hf on the Mo site, the $a_{1}^{\prime}$ level is lifted far above the $e^{\prime}$ levels and accommodates both holes so such substitutional impurities are nonmagnetic in the single impurity limit. Only when dopant pairs are sufficiently close $(\sim 6 \AA)$ does strong $\pi$ bonding lift the $e^{*}$ antibonding level above the $a$ bonding level so all four holes occupy antibonding states. The two holes in the $e^{*}$ states can become ferromagnetic with a total magnetic moment of $2 \mu_{B}$ but this is not an improvement on the single acceptor case.

In $\mathrm{MoSe}_{2}$ or $\mathrm{MoTe}_{2}$ (WSe $\mathrm{W}_{2}$ or $\mathrm{WTe}_{2}$ ) monolayers where the $\Gamma$ point VBM lies well below the $\mathrm{K} / \mathrm{K}^{\prime} \mathrm{VBM}$, the two holes introduced by double acceptors occupy $e^{\prime}$ 
states that can accomodate four holes. Half-filling of these degenerate levels leads to a competition between Jahn-Teller distortion and exchange splitting. If the JahnTeller distortion is sufficiently strong, the magnetic moment will be quenched and we do not expect double acceptors to be magnetic for low doping concentrations.

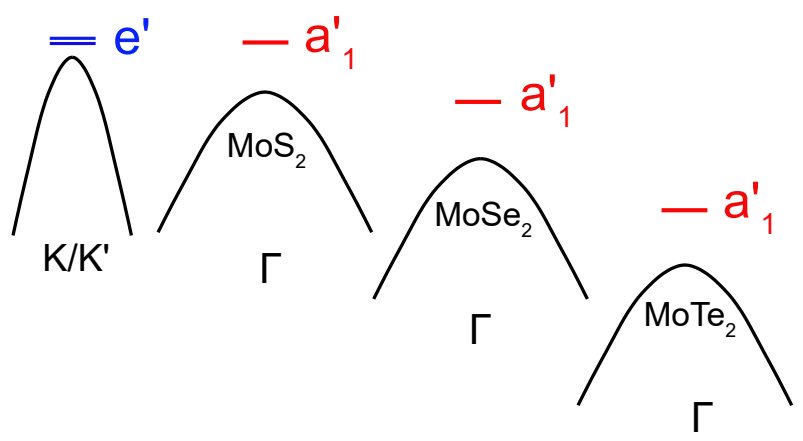

Figure 3.28: Schematic of the relative position of VBM at K and Gamma points and corresponding impurity levels for MX2.

\subsection{Summary \& Conclusions}

We have used $a b$ initio calculations to explore the possibility of inducing ferromagnetism in an $\mathrm{MoS}_{2}$ monolayer by substitutionally doping it with $\mathrm{V}$, Nb or Ta on Mo sites. In the single impurity limit, the resulting repulsive Coulomb potential leads to a doubly degenerate $\left\{d_{x y}, d_{x^{2}-y^{2}}\right\}$ state with $e^{\prime}$ symmetry bound to the $\mathrm{K} / \mathrm{K}^{\prime}$ valence band maxima and a singly degenerate $a_{1}^{\prime}$ state with $d_{3 z^{2}-r^{2}}$ character bound to the slightly lower-lying $\Gamma$ valence band maximum that are accidentally degenerate. The exchange interaction between two such hole states depends on whether the holes have $a_{1}^{\prime}$ or $e^{\prime}$ character, the former being quite localized, the latter quite extended in space. The magnetic moments of the spin $\frac{1}{2}$ acceptor states couple ferromagnetically at low concentrations but if the dopants are closer than the effective Bohr radius of the $d_{3 z^{2}-r^{2}}$ orbital, the magnetic moments quench in order to profit from the bonding interaction. The details of the exchange interaction depend sensitively on the equilibrium structure of the undoped monolayer that in turn depends on the (approximate) exchange-correlation functional used. We argue that the LDA is preferable to the GGA because it describes the ordering of the $\mathrm{K} / \mathrm{K}^{\prime}$ and $\Gamma$ valence band maxima better compared to experiment.

When spin-orbit coupling is included, we calculate a large magnetic anisotropy energy for acceptors with a preference for out of plane orientation and argue that this large single ion anisotropy justifies using an Ising spin model to study the ferromagnetic ordering. We estimate the ordering temperature by combining our 
(isotropic) separation-dependent exchange interactions with Monte Carlo calculations using Binder's cumulant method. For an $\mathrm{MoS}_{2}$ monolayer doped with $\mathrm{V}$ ( $\mathrm{Nb}$ or Ta), we estimate ferromagnetic Curie temperatures as a function of the dopant concentration and find a maximum $T_{C}$ of $\sim 170 \mathrm{~K}(\sim 100 \mathrm{~K})$ at around $9 \%$ dopant concentration. At sufficiently high concentrations of impurity states, the acceptor states form bands and magnetism is quenched when this band width exceeds a critical value that depends sensitively on the exchange-correlation functional used.

Although the maximum calculated $T_{C}$ is below room temperature, our work demonstrates that shallow impurities in $\mathrm{MX}_{2}$ monolayers that bind weakly but have long range interactions are promising dopants to explore with a view to realizing room temperature ferromagnetism.

\subsection{Appendices}

\subsubsection{Sulphur reference atom}

The impurity potential of a vanadium acceptor screened by a hole in the $a_{1}^{\prime}$ and $e^{\prime}$ states as felt by $S$ atoms is plotted in the insets to Fig. 3.29. The effective Bohr radii of these impurity states determined by fitting to Eq. (3.1b) are $8.8 \AA$ for screening by the $e^{\prime}$ state and $6.5 \AA$ for screening by the $a_{1}^{\prime}$ state. For the $e^{\prime}$ level, this effective Bohr radius is consistent with the other estimates summarized in Table 3.2. For the $a_{1}^{\prime}$ level, however, the value of $6.5 \AA$ yielded by the $S$ atom probes is larger than the value of $5.4 \AA$ yielded by using Mo atoms as probes. We already saw more scatter in the estimate of the $a_{1}^{\prime}$ radius and in view of its very small value and the importance of the central cell potential and local screening effects on this length scale, it is not surprising to see this type of variation measured by probes at different radial distances.

\subsubsection{Hydrogen atom in LDA/GGA}

In the local density approximation (LDA), the total energy of a neutral hydrogen atom is not -1 Rydberg but its absolute value is about $10 \%$ smaller, $\sim-0.89$ Rydberg and the Kohn-Sham eigenvalue for the $1 s$ state is $\varepsilon_{1 s}=-0.46$ [121]. It has been shown that the discrepancy can be substantially reduced by using the spinpolarized (SP) version of the LDA, the local spin density approximation (LSDA) [121]. Using the Perdew-Zunger [115] parameterization of L(S)DA (Table 3.9), we obtain total energies $(E)$ and KS eigenvalues $(\varepsilon)$ of $E=-0.89$ Ry, $\varepsilon=-0.46 \mathrm{Ry}$ (LDA) and $E=-0.97$ Ry (LSDA) and an exchange splitting of the Kohn-Sham $1 s$ eigenvalues of -0.35 Ry. If instead of the L(S)DA, we use the Perdew-BurkeErnzerhof [116] GGA, we obtain energies of -0.92 Ry (GGA) and -0.99 Ry (SP-GGA) and an exchange splitting of -0.55 Ry. 


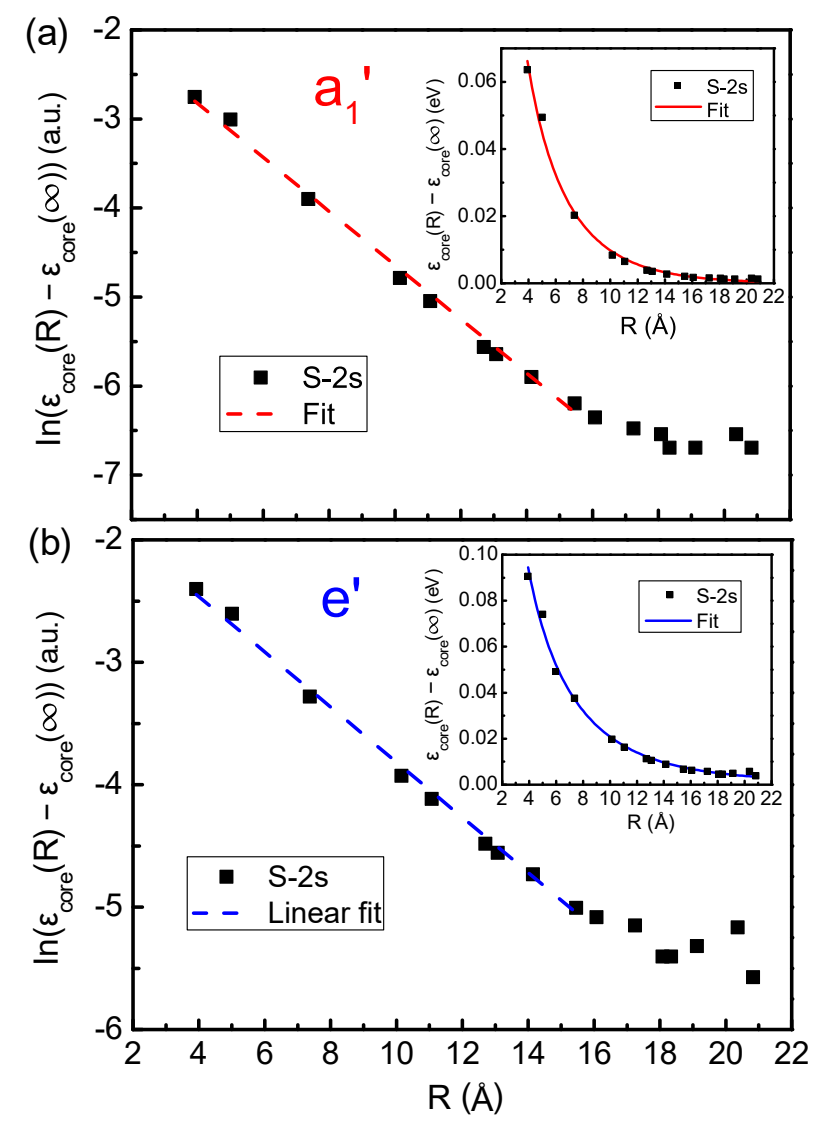

Figure 3.29: Dependence of the S $2 s$ semicore levels on the separation from the $\mathrm{V}_{\mathrm{Mo}}$ dopant ion. The (screened) Coulomb potential of the $\mathrm{V}$ dopant is screened by the $a_{1}^{\prime}$ hole (upper panel) respectively by the $e^{\prime}$ hole (lower panel). The asymptotic value $\varepsilon_{\text {core }}(\infty)$ was determined by fitting the calculated data points in the insets to an exponential wave function and using this fit (red curves) to extrapolate to $R=\infty$.

Table 3.9: Comparison of total energies $(E)$ and Kohn-Sham (KS) eigenvalues $(\varepsilon)$ for a hydrogen atom as described by the LDA and the LSDA, the non-spin polarized (NSP)-GGA and spin-polarized (SP)-GGA in Rydberg units (13.606 eV).

\begin{tabular}{lcclcc}
\hline & \multicolumn{2}{c}{ LDA } & & \multicolumn{2}{c}{ GGA } \\
\cline { 2 - 3 } \cline { 5 - 6 } & NSP & SP & & NSP & SP \\
\hline$E$ & -0.89 & -0.97 & & -0.92 & -0.99 \\
$\varepsilon_{1 s}^{\uparrow}$ & -0.46 & -0.18 & & -0.47 & 0.00 \\
$\varepsilon_{1 s}$ & -0.46 & -0.53 & & -0.47 & -0.55 \\
$\Delta \varepsilon_{1 s}$ & 0.00 & 0.35 & & 0.00 & 0.55 \\
\hline
\end{tabular}




\section{Systematic DFT study of structural and electronic properties in $p$-doped Monolayers of $\mathrm{MX}_{2}$}

We use density functional theory calculations to systematically investigate the structural and electronic properties of $M X_{2}$ transition metal dichalcogenide monolayers with $M=C r, M o, W$ and $X=S$, Se, Te that are doped with single $(V, N b, T a)$ and double (Ti, $\mathrm{Zr}, \mathrm{Hf}$ ) acceptor dopants on the $M$ site in the dilute limit. Three impurity levels are found above the valence band maxima (VBM). Under the local $D_{3 h}$ symmetry an orbitally doubly degenerate $e^{\prime}$ level is bound to the $K / K^{\prime} V B M$ and a singly degenerate $a_{1}^{\prime}$ level is bound to the $\Gamma$-point VBM. The relative positions of these impurity levels determines the different structural and electronic properties in p-doped $M X_{2}$ monolayers and can be tuned by replacing $S$ with $S e$ or Te for which the $\Gamma$ point VBM is substantially lower, bringing the $a_{1}^{\prime}$ level down with it.

For single acceptors, the near-degeneracy of the $a_{1}^{\prime}$ and $e^{\prime}$ levels in sulphur compounds is lifted for $X=$ Se and Te. As a result, the ground state of close impurity pairs is a triplet and the quenching of ferromagnetic coupling found for $X=S$ is avoided. Large out-of-plane magnetic anisotropy energies (as large as $10 \mathrm{meV} /$ dopant) are found in the single acceptor doped $M X_{2}$ systems, which is essential for long-range ferromagnetic ordering in two dimensions. However, when $X=S e$ and Te, a Jahn-Teller distortion lifts the degeneracy of the $e^{\prime}$ levels lowering the local symmetry of $\mathrm{V}, \mathrm{Nb}$ and Ta single acceptors and decreases the magnetic anisotropy energies.

For Ti, Zr and Hf double acceptors, both holes occupy the high lying $a_{1}^{\prime}$ level and so are not magnetic in $M S_{2}$ monolayers. In $M \mathrm{Se}_{2}$ and $M T e_{2}$ monolayers, the Jahn-Teller 
distortion competes with the exchange splitting and quenches the magnetic moment as it is large enough. Moreover, the double acceptors have a large in-plane magnetic anisotropy energy, which can not develop long-range magnetic ordering in $2 D$.

The existence of deep intrinsic vacancy and antisite defects would localize the holes and enhance the magnetization in p-doped $M X_{2}$ monolayers. Our systematic study on the p-doped $M X_{2}$ systems offers a guide to search for the most promising solution that realizes the room temperature ferromagnetic semiconductor in p-doped monolayers of $M X_{2}$.

\subsection{Introduction}

After ferromagnetism was observed in (In,Mn)As [57] and (Ga,Mn)As [58], predictions were made for achieving room temperature ordering [59] that sparked a huge effort to realize such a dilute magnetic semiconductor (DMS). In spite of twenty-five years of intensive research, the maximum ordering temperature has stagnated at values well below room temperature [60]. The number of material systems considered has proliferated but it is not clear what the fundamental limits are to the ordering temperature achievable in any particular material system, so research proceeds largely empirically. There are many reasons for the low ordering temperatures $[61 ; 62]$ but the essential dilemma is that the open $d$ shell states of magnetic impurities like Mn are quite localized. While this favours the onsite exchange interaction that is the origin of the Hund's-rule spin alignment and makes the ionic moment insensitive to temperature, it leads to weaker exchange interactions between pairs of impurity ions that determine the Curie temperature $T_{C}$, the ferromagnetic ordering temperature. $T_{C}$ can be increased by increasing the concentration of impurity atoms but this is accompanied by adverse effects such as the clustering of magnetic impurities or the formation of antisite defects that are electron donors which counter the intended increase in the concentration of holes. In many semiconductors, transition metal ions like Mn, Fe or Co introduce "deep levels", tightly bound partially occupied states, in the fundamental gap of the semiconductor. At high dopant concentrations, these form deep impurity bands that dominate the (transport) properties of a material that from the electronic structure point of view is no longer a semiconductor but an entirely new material.

We recently explored a different approach to realizing a high temperature magnetic semiconductor $[106 ; 158]$. In a tight-binding approach, the transition metal (TM) $d$ bandwidth is $2 z t$ where $z$ is the number of neighbouring TM atoms and $t$ is the nearest neighbour hopping matrix element. Because of the reduced coordination number $z$, a two-dimensional material like the TM dichalcogenide $\mathrm{MoS}_{2}$ has a substantially reduced $d$ bandwidth $W_{d}$; this is favourable for the occurrence of itinerant ferromagnetism as the Stoner criterion $D\left(E_{F}\right) I_{\mathrm{xc}}>1$ is more easily satisfied where $D\left(E_{F}\right)$ is the density of states (DoS) at the Fermi level and $I_{\mathrm{xc}}$ is the Stoner 
parameter. $I_{\mathrm{xc}}$ is essentially an atomic property and in the local spin density approximation (LSDA) [159; 126] it contains contributions from both exchange and correlation $[160 ; 161 ; 127]$.

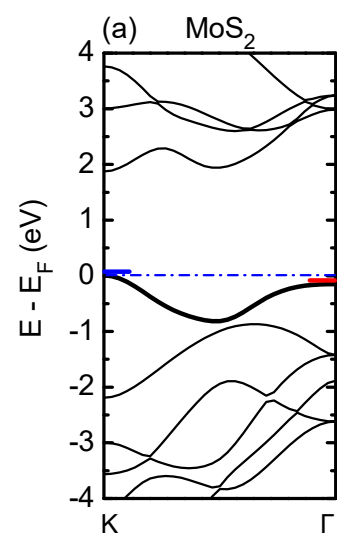

(b) $\mathrm{MoSe}_{2}$

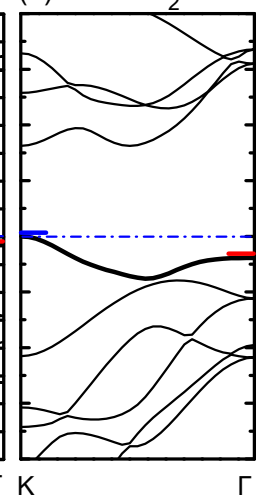

(d) $\mathrm{MoS}_{2}: 1 \mathrm{~V}$

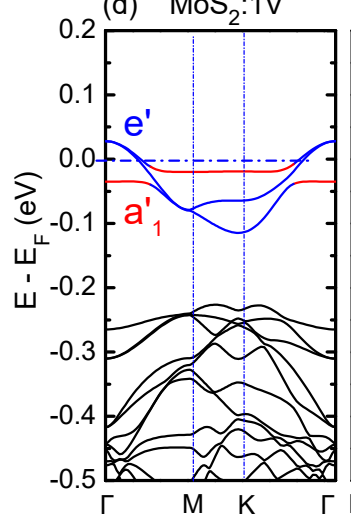

(e) $\mathrm{MoSe}_{2}: 1 \mathrm{~V}$

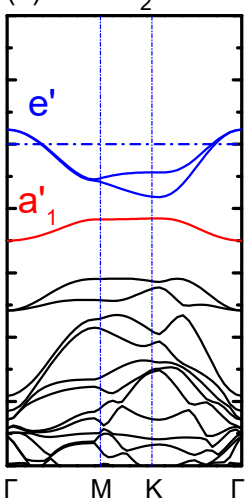

(c) $\mathrm{MoTe}_{2}$

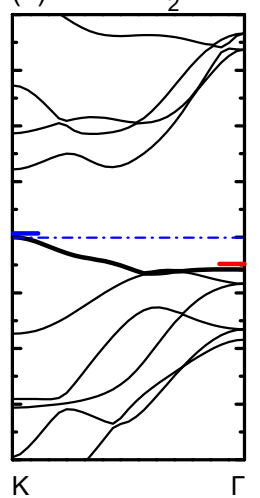

(f) $\mathrm{MoTe}_{2}: 1 \mathrm{~V}$

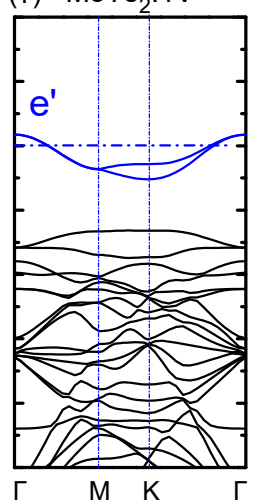

Figure 4.1: (color online). Band structures of monolayer $\mathrm{MoX}_{2}(\mathrm{X}=\mathrm{S}, \mathrm{Se}, \mathrm{Te})$ without (a-c) and with (d-f) a single substitutional vanadium dopant atom on a Mo site in a $6 \times 6$ supercell. The zero of energy is the Fermi level. In (a-c), the blue and red horizontal lines represent, respectively, the $e^{\prime}$ impurity levels bound to the $K / K^{\prime}$ valence band maxima (VBM) and the $a_{1}^{\prime}$ impurity level bound to the $\Gamma$ VBM. In (d-f) the blue and red lines are the corresponding bands formed from these impurity levels because of the finite size of the unit cell and the periodic boundary conditions. Note the different energy scales used in the top and bottom panels.

A monolayer of $\mathrm{MoS}_{2}$ is a direct gap semiconductor with a very narrow nonbonding Mo $d$ band, the bold black band in Fig. 4.1(a), forming the top of the valence band in a gap formed by bonding and antibonding combinations of Mo- $d$ and S- $p$ states. To probe the enhanced DoS, we considered hole-doping $\mathrm{MoS}_{2}$ by replacing some of the Mo atoms with group VB acceptor atoms in Ref.106; 158. Mo has a $4 d^{5} 5 s^{1}$ electronic configuration and in $\mathrm{MoS}_{2}$ it is nominally $\mathrm{Mo}^{4+}$ with one 
up-spin and one down-spin $d$ electron so it is nonmagnetic. When a group VIB Mo is replaced by a group $\mathrm{VB}$ atom like $\mathrm{V}, \mathrm{Nb}$ or $\mathrm{Ta}$, then the dopant atom e.g. $\mathrm{V}^{4+}$, has a single unpaired $d$ electron and a single hole is thereby introduced into the narrow Mo $4 d$ band; substitution of a group IVB atom (Ti, Zr, Hf) will introduce two holes per dopant atom. In the single impurity limit, the asymptotic Coulomb potential leads to a series of hydrogenic states bound to the top of the valence band. At the $K$ and $K^{\prime} \mathrm{VBM}$, the highest doubly degenerate state with $e^{\prime}$ symmetry in the local $D_{3 h}$ symmetry has mixed Mo $d_{x^{2}-y^{2}}$ and $d_{x y}$ character. At the slightly lower $\Gamma$-point VBM the highest $a_{1}^{\prime}$ state has Mo $d_{3 z^{2}-r^{2}}$ character and a large effective mass [107]. At finite concentrations of dopant atoms, these impurity states form impurity bands and at finite temperatures, the holes in these impurity bands are thermally excited into the top of the narrow nonbonding Mo $d$ band.

In Ref. 106; 158 we found that pairs of $\mathrm{V}, \mathrm{Nb}$ and Ta substitutional dopants in an $\mathrm{MoS}_{2}$ monolayer coupled ferromagnetically for all dopant separations but the shortest. In the single impurity limit, a large out-of-plane magnetic anisotropy of $\sim 5 \mathrm{meV}$ per dopant ion was found that allows violation of the Mermin-Wagner theorem according to which thermal fluctuations destroy ordering in two dimensions for isotropic Heisenberg exchange [63; 64]. Large single ion anisotropy (SIA) combined with isotropic exchange coupling leads to effective Ising-spin behaviour [142] and long-range magnetic ordering $[130 ; 131]$ at finite ordering temperatures that can be estimated using Monte-Carlo calculations [143; 144]. For V, Nb and Ta, we estimated values of $T_{C}$ of about $100 \mathrm{~K}$ for $\mathrm{Nb}$ and Ta and as high as $160 \mathrm{~K}$ for $\mathrm{V}$ for doping concentrations of order $9 \%$. Very recent reports of long-range and/or room temperature ferromagnetism occurring in $\mathrm{V}$ doped $\mathrm{WSe}_{2}$ monolayers [153], in $\mathrm{MoSe}_{2}$ and $\mathrm{MoTe}_{2}$ [154], in V and Ta doped $\mathrm{MoTe}_{2}$ [155; 156] and in $\mathrm{MoS}_{2}$ [157] (whereby the interaction with anion vacancies would appear to play an important role) provide the motivation for a closer study of these systems.

In our study of $\mathrm{MoS}_{2}$, we identified the formation of singlet states for dopant pairs closer than a critical separation $(\sim 9 \AA)$ as the most important factor limiting the ordering temperature to below room temperature in $p$-doped $\mathrm{MoS}_{2}$ monolayers [106; 158]. For close dopant pairs, the $\pi$ interaction of $a_{1}^{\prime}$ states with $d_{3 z^{2}-r^{2}}$ character is stronger than the $\sigma$ interaction between $e^{\prime}$ states with $d_{x^{2}-y^{2}}$ and $d_{x y}$ character so the highest lying level occupied by the two holes is an antibonding $a^{*}$ state. It is orbitally nondegenerate so the holes are forced to have opposite spins i.e., the net magnetic moment is zero. Because of their $d_{3 z^{2}-r^{2}}$ character, the $a_{1}^{\prime}$ states are very localized in the $x y$ plane and the interaction between $a_{1}^{\prime}$ states decays more rapidly than that between $e^{\prime}$ states with $d_{x^{2}-y^{2}}$ and $d_{x y}$ character. For separations larger than the critical distance, the orbitally degenerate antibonding $e^{*}$ state can accomodate two holes with parallel spins corresponding to a ferromagnetic exchange interaction. Because the $\Gamma$-point VBM is so much lower for $\mathrm{MoSe}_{2}$ and $\mathrm{MoTe}_{2}$, compare Fig. 4.1(a)-(c), we expect the $a_{1}^{\prime}$ levels will lie correspondingly low in energy and be fully occupied so quenching of the ferromagnetic interaction 
will not occur. However, there is a downside. Because of its spatial localization, the exchange splitting of the $a_{1}^{\prime}$ levels is substantially larger than that of the $e^{\prime}$ levels making it much less sensitive to electronic thermal excitation. Because it is not a priori clear where the optimum interatomic exchange coupling and ordering temperature will occur, the purpose of this manuscript is to perform a systematic study of dopant acceptor levels in $\mathrm{MX}_{2}$ monolayers with $\mathrm{M}=\mathrm{Cr}$, Mo, $\mathrm{W} ; \mathrm{X}=\mathrm{S}$, Se, Te in the single impurity limit. Because of the Mermin-Wagner theorem we will also evaluate the magnetic anisotropy in this same limit. Intrinsic defects such as vacancies and antisite defects are commonly observed in various van der Waals materials. Because the interplay between substitutional $\mathrm{M}$ dopants and intrinsic defects could have significant influence on the Curie temperature, we will also consider some representative examples of these.

This Chapter is organized as follows. After a short summary of some computational details in Sec. 4.2, we begin Sec. 4.3 with a brief comparison of the calculated structural and electronic properties of pristine $\mathrm{MX}_{2}$ monolayers in Sec. 4.3.1. Next we report the structural and electronic properties in p-doped $\mathrm{MX}_{2}$ monolayers, including dopant binding energy, John-Teller distortion, magnetic anisotropy energy. Finally, the effect of intrinsic defects and the solution for room temperature ferromagnetic semiconductor are discussed.

\subsection{Computational Method}

First-principles density functional theory (DFT) calculations were performed with a plane-wave basis and the projector augmented wave (PAW) formalism [109] as implemented in the vASP code $[110 ; 111 ; 112]$. An energy cut-off of $400 \mathrm{eV}$ was used and atomic structures were fully relaxed until all forces were less than 0.01 $\mathrm{eV} / \AA$. Monolayers of $\mathrm{MX}_{2}$ periodically repeated in the $c$ direction were separated by more than $20 \AA$ of vacuum to avoid spurious interaction. Because we only consider free-standing monolayers, there was no need to take van der Waals interactions into account. The local (spin) density approximation, L(S)DA, underestimates equilibrium atomic separations more than generalized gradient approximations (GGA) overestimate them. However, by comparison with experiment, the LDA describes the relative positions of the valence band maxima better than does the GGA $[106 ; 158]$. Since this will play an important role in our study of shallow impurity levels, we used the LSDA as parameterized by Perdew and Zunger [115]. A $12 \times 12$ supercell was used to reduce the interaction between neighboring supercells to an acceptable degree when modelling dopants in the single impurity limit. A $2 \times 2 \mathrm{k}$-point sampling was used for structure relaxation and $4 \times 4$ for the calculations with spin-polarization and spin orbit coupling. For intrinsic defects, a smaller $6 \times 6$ supercell was found to be sufficient to reliably describe the defect potential inside the supercell because of the greater localization of the gap states. 
Table 4.1: Structural and electronic properties of $\mathrm{MX}_{2}$ monolayers. Shown are the lattice constant $a(\AA)$, the separation of two chalcogen X layers $c_{\mathrm{X}}$, band gap $\Delta \varepsilon_{g}(\mathrm{eV})$, energy difference at $K$ and $\Gamma$ valley $\Delta_{K \Gamma}(\mathrm{eV})$, and the bandwidth $W$ of the "nonbonding" band forming the top of the valence band.

\begin{tabular}{|c|c|c|c|c|c|c|c|c|c|c|c|c|c|c|c|}
\hline $\mathrm{X}$ & & & S & & & & & $\mathrm{Se}$ & & & & & $\mathrm{Te}$ & & \\
\hline $\mathrm{M}$ & $a$ & $c_{\mathrm{X}}$ & $\Delta \varepsilon_{g}$ & $\Delta_{K \Gamma}$ & $W$ & $a$ & $c_{\mathrm{X}}$ & $\Delta \varepsilon_{g}$ & $\Delta_{K \Gamma}$ & $W$ & $a$ & $c_{\mathrm{X}}$ & $\Delta \varepsilon_{g}$ & $\Delta_{K \Gamma}$ & $W$ \\
\hline $\mathrm{Cr}$ & 2.97 & 2.92 & 1.07 & 0.22 & 0.95 & 3.12 & 3.11 & 0.87 & 0.32 & $0.86^{-}$ & 3.37 & 3.38 & 0.62 & 0.45 & 0.70 \\
\hline Calc Mo & 3.12 & 3.11 & 1.87 & 0.15 & 1.06 & 3.25 & 3.31 & 1.62 & 0.38 & 1.02 & 3.47 & 3.59 & 1.22 & 0.58 & 0.86 \\
\hline $\mathrm{W}$ & 3.12 & 3.12 & 2.01 & 0.17 & 1.30 & 3.25 & 3.31 & 1.72 & 0.43 & 1.22 & - & - & - & - & - \\
\hline $\mathrm{Cr}$ & - & - & - & - & - & - & - & - & - & - & - & - & - & - & - \\
\hline Expt Mo & $3.16^{a}$ & $3.17^{a}$ & $1.90^{b}$ & $0.14^{c}$ & - & $3.29^{a}$ & $3.34^{a}$ & $1.58^{d}$ & $0.44^{e}$ & $0.90^{e}$ & $3.52^{f}$ & $3.60^{f}$ & $1.10^{g}$ & - & - \\
\hline $\mathrm{W}$ & $3.15^{h}$ & $3.14^{h}$ & $2.01^{i}$ & $0.24^{j}$ & - & $3.28^{h}$ & $3.34^{h}$ & $1.66^{k}$ & $0.50^{e}$ & $1.24^{e}$ & - & - & - & - & - \\
\hline
\end{tabular}

\subsection{Results}

\subsection{1 $\mathrm{MX}_{2}$ Monolayers}

We start by considering the structural and electronic properties of pristine $\mathrm{MX}_{2}$ monolayers with $\mathrm{M}=\mathrm{Cr}$, Mo, $\mathrm{W}$ and $\mathrm{X}=\mathrm{S}$, Se, Te. As sketched in Fig. 4.2, monolayers of these $\mathrm{MX}_{2}$ compounds have hexagonal lattices with honeycomb structures in which a one atomic thick layer of transition metal atoms is sandwiched between atomic layers of chalcogen atoms. The $\mathrm{M}$ atoms sit at the center of a triangular prism of $\mathrm{X}$ atoms in a so-called " $\mathrm{H}$ " structure. In metallic $\mathrm{MX}_{2}$ compounds, the $\mathrm{M}$ atoms sit at the center of an octahedron of $\mathrm{X}$ atoms in a so-called "T" struc-

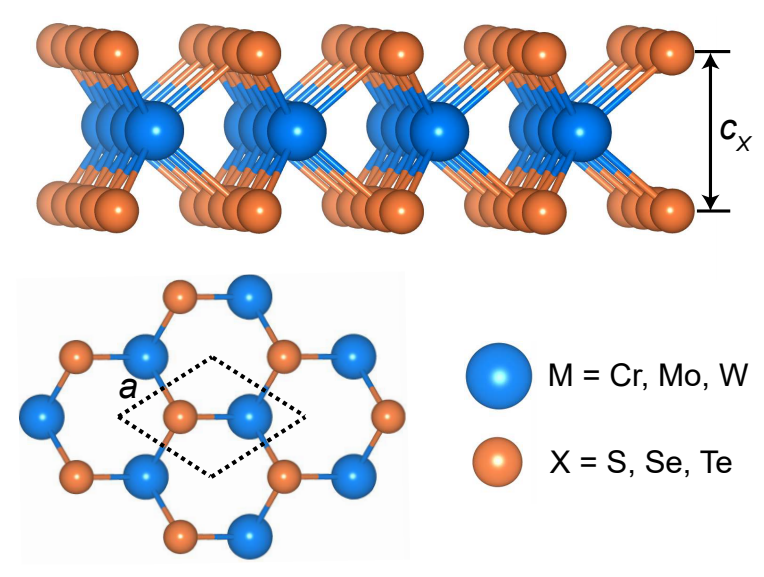

Figure 4.2: (color online). Side view and top view of the atomic structure of $\mathrm{MX}_{2}$ $(\mathrm{M}=\mathrm{Cr}, \mathrm{Mo}, \mathrm{W} ; \mathrm{X}=\mathrm{S}, \mathrm{Se}, \mathrm{Te})$ monolayer. The unit cell is enclosed by the dotted lines. 
ture. Under ambient conditions, all group VI TMDs are reported to have $\mathrm{H}$ phase structure. Even though the stable structure of $\mathrm{CrX}_{2}$ is still the subject of discussion $[171 ; 172 ; 173 ; 174]$, the $\mathrm{CrS}_{2}$ and $\mathrm{CrSe}_{2} 1 \mathrm{H}$ phases can be stabilized by choosing appropriate growth conditions [175]. WTe 2 is found to be semi-metallic with a $T_{d}$ polytype structure [176] and will not be considered further in this manuscript.

The equilibrium structural and electronic parameters for $1 \mathrm{H}-\mathrm{MX}_{2}$ monolayers as calculated in the LDA [115] are listed in Table 4.1. 1H corresponds to one layer per hexagonal unit cell as in monolayers of $\mathrm{MX}_{2}$. For bulk $\mathrm{MX}_{2}$, the hexagonal unit cell has two layers indicated by $2 \mathrm{H}$. In the $\mathrm{MoX}_{2}$ monolayers, the lattice constant $a$ is seen to increase as $\mathrm{X}$ goes from $\mathrm{S}$ to Se to Te. This leads to a reduction in the overlap of Mo $d$ orbitals and consequently of the bandwidth $W$ of the "nonbonding" $d$ band that forms the topmost valence band in Fig. 4.1(a)-(c). The direct band gap $\Delta \varepsilon_{g}$ at the $K$ point decreases from $\mathrm{MoS}_{2}$ to $\mathrm{MoTe}_{2}$, while the energy difference $\Delta_{K \Gamma}$ between the $K / K^{\prime}$ and $\Gamma \mathrm{VBM}$ associated with the ratio $a / c_{\mathrm{X}}$ increases in the same sequence. The eigenstates at the $K / K^{\prime}$ VBM have mixed in-plane $d_{x^{2}-y^{2}}$ and $d_{x y}$ character which is sensitive to the in-plane lattice constant $a$. As $a$ increases, the reduced overlap of Mo in-plane $d_{x^{2}-y^{2}}$ and $d_{x y}$ orbitals lowers the eigenvalues at the $K / K^{\prime}$ VBM. For the eigenstates at the $\Gamma$ point that have $d_{3 z^{2}-r^{2}}$ character, the separation $c_{\mathrm{X}}$ of the two chalcogen $\mathrm{X}$ layers determines the overlap between the $\mathrm{X} p$ orbitals and the $\mathrm{M} d_{3 z^{2}-r^{2}}$ orbital. The eigenvalues at the $\Gamma$ point would also be lowered with increasing $c_{\mathrm{X}}$. The ratio $a / c_{\mathrm{X}}$ can qualitatively indicate the size of $\Delta_{K \Gamma}$.

As $\mathrm{X}$ goes from $\mathrm{S} \rightarrow \mathrm{Te}, \mathrm{CrX}_{2}$ and $\mathrm{WX}_{2}$ monolayers exhibit similar trends. Compared to $\mathrm{MoX}_{2}, \mathrm{CrX}_{2}$ has a smaller lattice constant $a$ and that of $\mathrm{WX}_{2}$ is similar because of the similar radii of Mo and $\mathrm{W}$ atoms. The bandwidth $W$ increases as $\mathrm{M}$ goes from $\mathrm{Cr}$ to $\mathrm{W}$, which is attributed to the significant increase of electronegativity from $\mathrm{Cr}$ to $\mathrm{W}$ that increases the overlap of $\mathrm{M} d$ orbitals. It should be noted that the overlap of $\mathrm{M} d$ orbitals that determines the bandwidth $W$ is not only related to the M-M bond length but also largely attributed to the electronegativity of metal atoms that determines the total charge attracted around $\mathrm{M} d$ orbitals. The band gap $\Delta \varepsilon_{g}$ also increases with increasing atomic number of $\mathrm{M}$ and $\Delta_{K \Gamma}$ exhibits a similar trend except for $\mathrm{CrS}_{2}$. The properties of the dopant are largely determined by their host. In the next section, we will investigate how dopant properties depend on their host material.

\subsubsection{Structural and electronic properties of $\mathrm{p}$-doped $\mathrm{MX}_{2}$ monolayers}

\section{Formation energy}

The formation energy of an impurity determines how easily doping is achieved and is here defined as

$$
E_{\text {form }}=E_{\mathrm{tot}}\left[\mathrm{MX}_{2}: \text { dopant }\right]-E_{\mathrm{tot}}\left[\mathrm{MX}_{2}\right]+\sum_{i} n_{i} \mu_{i}
$$


where $E_{\text {tot }}\left[\mathrm{MX}_{2}\right.$ : dopant $]$ is the total energy of a relaxed supercell of an $\mathrm{MX}_{2}$ monolayer with a single $\mathrm{M}$ atom replaced with a dopant atom, $E_{\mathrm{tot}}\left[\mathrm{MX}_{2}\right]$ is the total energy of a pristine $\mathrm{MX}_{2}$ monolayer and $\mu_{i}$ is the bulk chemical potential of the added $\left(n_{i}=+1\right)$ or removed $\left(n_{i}=-1\right)$ atom.

The negative formation energies listed in Table 4.2 indicate that substitutional doping of group IVB and VB elements in monolayers of $\mathrm{MX}_{2}$ is energetically favorable. For $\mathrm{MoX}_{2}$, the formation energy increases from $\mathrm{V}$ to Ta, reaches the peak for $\mathrm{Ti}$ and then is reduced for $\mathrm{Zr}$ and $\mathrm{Hf}$. In general, the double acceptors have larger formation energy than the single acceptors. $\mathrm{CrX}_{2}$ and $\mathrm{WX}_{2}$ exhibit similar trends. Compared to $\mathrm{MoX}_{2}$ and $\mathrm{WX}_{2}$, the single acceptors in $\mathrm{CrX}_{2}$ have much larger formation energies.

Table 4.2: Calculated formation energy (eV) of various dopant in $\mathrm{MX}_{2}$ monolayer with relaxation.

\begin{tabular}{lcccccccc}
\hline & $\mathrm{CrS}_{2}$ & $\mathrm{MoS}_{2}$ & $\mathrm{WS}_{2}$ & $\mathrm{CrSe}_{2}$ & $\mathrm{MoSe}_{2}$ & $\mathrm{WSe}_{2}$ & $\mathrm{CrTe}_{2}$ & $\mathrm{MoTe}_{2}$ \\
\hline $\mathrm{V}$ & -0.26 & 0.25 & 0.24 & -0.37 & 0.32 & -0.07 & -0.45 & 0.22 \\
$\mathrm{Nb}$ & -0.25 & -0.15 & -0.10 & -0.55 & -0.14 & -0.49 & -0.62 & 0.03 \\
$\mathrm{Ta}$ & -0.36 & -0.23 & -0.02 & -0.43 & -0.10 & -0.39 & -0.57 & 0.07 \\
$\mathrm{Ti}$ & -0.32 & -0.58 & -0.64 & -0.74 & -0.57 & -0.72 & -0.62 & -0.33 \\
$\mathrm{Zr}$ & -0.25 & -0.25 & -0.21 & -0.46 & -0.27 & -0.55 & -0.56 & -0.21 \\
$\mathrm{Hf}$ & -0.42 & -0.32 & -0.39 & -0.52 & -0.31 & -0.84 & -0.59 & -0.42 \\
\hline
\end{tabular}

\section{Binding energy}

On substituting an $\mathrm{M}$ atom $(\mathrm{M}=\mathrm{Cr}, \mathrm{Mo}, \mathrm{W})$ in an $\mathrm{MX}_{2}$ monolayer with a single (V, Nb, Ta) or a double (Ti, Zr, Hf) acceptor in the single impurity limit, intervalley scattering leads to orbitally twofold degenerate $e^{\prime}$ and singly degenerate $a_{1}^{\prime}$ levels bound to the $K / K^{\prime}$ valley and $\Gamma$ valley maxima, respectively. In effective mass theory (EMT), a Coulomb potential leads to the formation of a Rydberg series of (shallow impurity) levels bound to the valence band maxima [117; 118; 119]. The impurity binding energy is defined as the position of the level with respect to the VBM and determines the degree of localization of the impurity wavefunction.

To determine the position of impurity levels with respect to the VBM, we first need to be able to identify the VBM in a doped systems, something which is not trivial in a supercell calculation because of the interaction of Coulomb bound states with their periodic images over all length scales [158]. Because the binding energy of both single and double acceptors in $\mathrm{MX}_{2}$ monolayers is much smaller than the sizeable bandgap, see Fig. 4.3, and a repulsive Coulomb potential does not affect the conduction band states, in particular the states forming the conduction band minima (CBM), we can determine the bulk VBM in a doped system from its position 


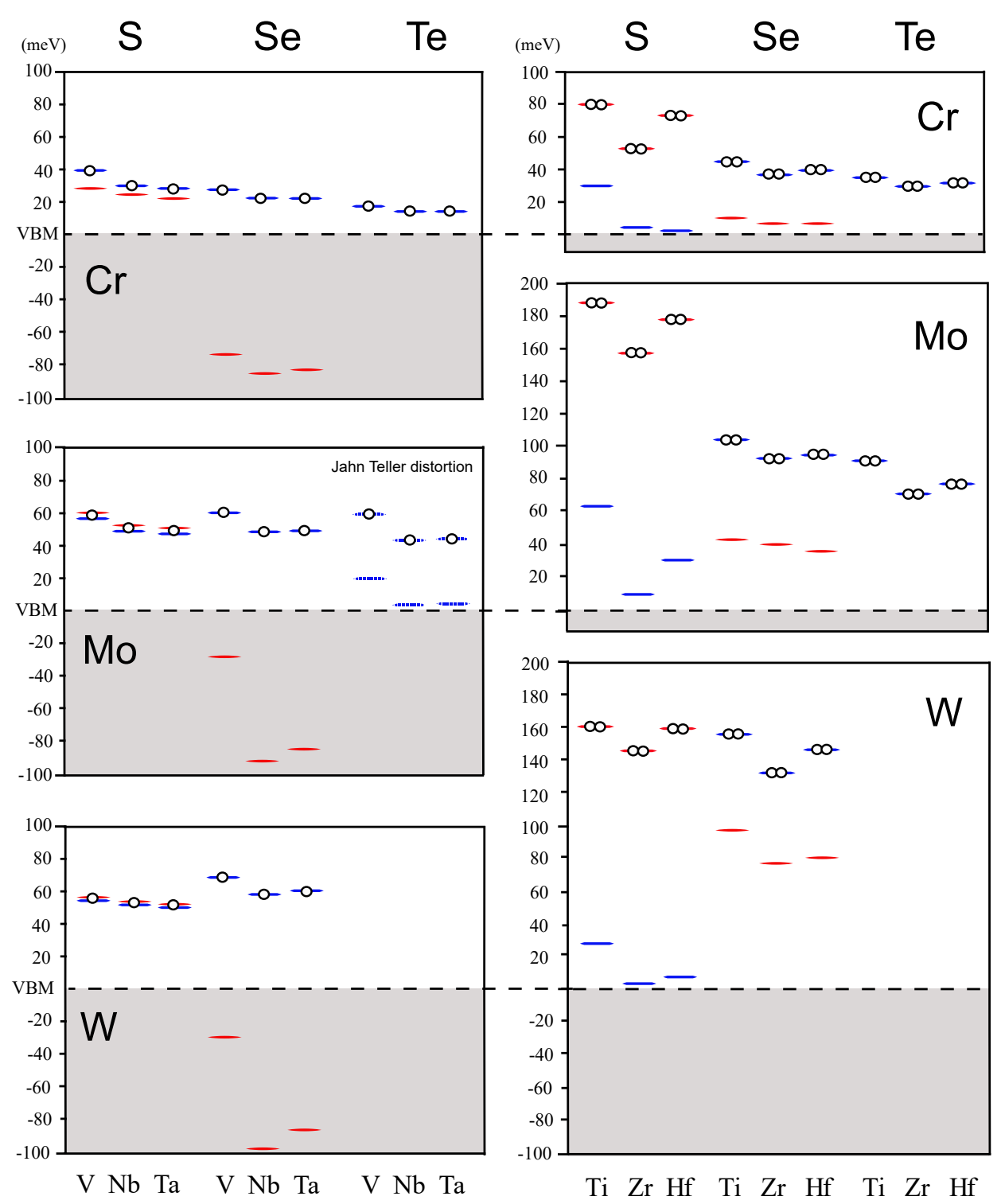

Figure 4.3: (color online). Impurity levels for single (V, Nb, Ta) and double ( $\mathrm{Ti}$, $\mathrm{Zr}, \mathrm{Hf}$ ) acceptors in $\mathrm{MX}_{2}$ monolayers including atomic relaxation but without spin polarization. The red lines represent $a_{1}^{\prime}$ levels, the blue lines represent degenerate $e^{\prime}$ levels. The dashed blue lines indicate the degeneracy lifted $e^{\prime}$ levels due to the Jahn Teller distortion. Holes represented by open circles occupy the highest levels in each system. 
relative to the $\mathrm{CBM}$ in the pristine case $\left(\varepsilon_{\mathrm{VBM}}=\varepsilon_{\mathrm{CBM}}-\varepsilon_{g}\right)$. Alternatively, we can align the VBM to a core level of an atom far from the dopant site, e.g., the Mo $4 s$ level, extrapolating to infinite separation and relating that to the corresponding energy separation of the core level and VBM in a pristine $\mathrm{MX}_{2}$ monolayer. The two methods give consistent results [158]. For convenience, we use the first to extract the position of the VBM. The position of single (V, Nb, Ta) and double (Ti, $\mathrm{Zr}$, Hf) acceptor impurity levels with respect to the VBM in doped $\mathrm{MX}_{2}$ monolayer determined in this way are shown in Fig. 4.3. Because of the finite dispersion of the impurity "bands" in a supercell calculation, what is shown is the appropriate weighted average over the Brillouin-zone of the impurity band.

As already shown in Chapter 3 for monolayers of $\mathrm{MoS}_{2}$ where the $a_{1}^{\prime}$ and $e^{\prime}$ levels are almost degenerate, the binding energy of single acceptor levels decreases from $\mathrm{V}$ to Ta as shown in Fig. 4.3. The energy difference $\Delta_{K \Gamma}$ between the $K / K^{\prime}$ and $\Gamma$ VBM in pristine $\mathrm{MX}_{2}$ monolayers determines the relative position of the impurity levels bound to these VBM. As tabulated in Table 4.1, $\Delta_{K \Gamma}$ increases as X goes from $\mathrm{S}$ to $\mathrm{Te}$, so the $a_{1}^{\prime}$ levels (red lines) drop fast with respect to the $e^{\prime}$ levels (blue lines) going from $\mathrm{MoS}_{2}$ to $\mathrm{MoTe}_{2}$ as documented in Fig. 4.3. The $e^{\prime}$ levels drop slightly from $\mathrm{MoS}_{2}$ to $\mathrm{MoTe}_{2}$. In $\mathrm{MoTe}_{2}$, a Jahn-Teller distortion splits the degenerate $e^{\prime}$ levels; we return to this below. The impurity binding energy in single acceptor doped $\mathrm{CrX}_{2}$ monolayers shows a similar trend to that in $\mathrm{MoX}_{2}$ but with a shallower binding energy, while in $\mathrm{WX}_{2}$ the impurity binding energy is almost the same as in $\mathrm{MoX}_{2}$. For the double acceptors, the main trends of $a_{1}^{\prime}$ and $e^{\prime}$ levels in $\mathrm{MX}_{2}$ are the same as the case with single acceptors. The main difference is that the $a_{1}^{\prime}$ levels in $\mathrm{MS}_{2}$ that accommodate both holes lie well above the $e^{\prime}$ levels.

\section{Spin polarization}

The result of calculating the magnetic moments of $\mathrm{MX}_{2}$ monolayers with different dopants with full structural relaxation in $12 \times 12$ supercells is given in Table 4.3. In the single impurity limit, the magnetic moments are expected to be an integer number of Bohr magnetons, $\mu_{B}$. For all three single acceptors $\mathrm{V}, \mathrm{Nb}$, and $\mathrm{Ta}$, this is true and $\mathrm{MoS}_{2}$ is fully polarized. The same is true for $\mathrm{MoSe}_{2}$ and $\mathrm{MoTe}_{2}$. Single acceptor doped $\mathrm{CrX}_{2}$ monolayers also have a magnetic moment of $1 \mu_{B}$ for all three single acceptors. Even though the defect levels of $\mathrm{Nb}$ and $\mathrm{Ta}$ acceptors in $\mathrm{WX}_{2}$ are as deep as in $\mathrm{MoX}_{2}$ and deeper than in $\mathrm{CrX}_{2}$, see Fig. 4.3, their magnetic moments are less than $1 \mu_{B}$. Since the impurities must be fully polarized in the single impurity limit, we must attribute this partial polarization to the finite $12 \times 12$ supercell size not being sufficiently large to avoid significant interaction between dopants in neighbouring cells. Even if dopants in $\mathrm{WX}_{2}$ are fully polarized in the single impurity limit, their exchange splitting may be smaller. As we discussed above, the large electronegativity of $\mathrm{W}$ leads to enhanced hybridization between $\mathrm{W} d$ orbitals. Similarly, the hybridization between impurity states and $\mathrm{W} d$ orbitals makes the 
Table 4.3: Calculated magnetic moments in $\mu_{B}$ for monolayers of $\mathrm{MX}_{2}(12 \times 12$ supercell) doped with various transition metal atoms substituting a single Mo. The structures are fully relaxed.

\begin{tabular}{lcccccccc}
\hline & $\mathrm{CrS}_{2}$ & $\mathrm{MoS}_{2}$ & $\mathrm{WS}_{2}$ & $\mathrm{CrSe}_{2}$ & $\mathrm{MoSe}_{2}$ & $\mathrm{WSe}_{2}$ & $\mathrm{CrTe}_{2}$ & $\mathrm{MoTe}_{2}$ \\
\hline $\mathrm{V}$ & 1.00 & 1.00 & 1.00 & 1.00 & 1.00 & 1.00 & 1.00 & 1.00 \\
$\mathrm{Nb}$ & 1.00 & 1.00 & 0.91 & 1.00 & 1.00 & 0.71 & 1.00 & 1.00 \\
$\mathrm{Ta}$ & 1.00 & 1.00 & 0.90 & 1.00 & 1.00 & 0.70 & 1.00 & 1.00 \\
\hline $\mathrm{Ti}$ & 0.00 & 0.00 & 0.00 & 2.00 & 2.00 & 2.00 & 2.00 & 2.00 \\
$\mathrm{Zr}$ & 0.00 & 0.00 & 0.00 & 2.00 & 2.00 & 2.00 & 2.00 & 2.00 \\
$\mathrm{Hf}$ & 0.00 & 0.00 & 0.00 & 2.00 & 2.00 & 2.00 & 2.00 & 2.00 \\
\hline
\end{tabular}

impurity states more delocalized.

The double acceptor impurities are all fully polarized with magnetic moments of $2 \mu_{B}$ except for the $\mathrm{MS}_{2}$ monolayers with $\mathrm{M}=\mathrm{Cr}$, Mo, W. In these systems, the $a_{1}^{\prime}$ level lies well above the $e^{\prime}$ levels, as seen in Fig. 4.3, leaving two holes on the singly degenerate $a_{1}^{\prime}$ level that quenches the magnetic moment. In the $\mathrm{MSe}_{2}$ and $\mathrm{MTe}_{2}$ monolayers, the holes are accommodated in the doubly degenerate $e^{\prime}$ levels that need not and does not quench the magnetic moment. However, the half-filled orbitally degenerate state is susceptible to a Jahn-Teller (JT) distortion leading to a competition between exchange interaction and JT distortion. If the JT distortion is strong enough, the magnetic moment will be quenched. We discuss this in the next section.

The exchange splittings listed in Table 4.4 depend on the (relative) positions of the $a_{1}^{\prime}$ and $e^{\prime}$ levels and on the localization of these states. The exchange splitting of the $a_{1}^{\prime}$ level is relatively large because of the spatial localization of this state while a much smaller exchange splitting is associated with the much more delocalized $e^{\prime}$ levels. The relative position of $a_{1}^{\prime}$ and $e^{\prime}$ levels determines the hole occupation of these two impurity levels and so the total spin polarization. The high-lying localized $a_{1}^{\prime}$ level enhances the exchange splitting of dopants in $\mathrm{MS}_{2}$ systems, while

Table 4.4: Calculated exchange splitting $\Delta$ in meV for monolayers of $\mathrm{MX}_{2}(12 \times 12$ supercell) doped with $\mathrm{V}$ and $\mathrm{Ti}$ substituting a single $\mathrm{Cr} / \mathrm{Mo} / \mathrm{W}$.

\begin{tabular}{|c|c|c|c|c|c|c|c|c|c|}
\hline \multirow{2}{*}{\multicolumn{2}{|c|}{$\begin{array}{l}M \\
X\end{array}$}} & $\mathrm{Cr}$ & Mo & W & $\mathrm{Cr}$ & Mo & W & $\mathrm{Cr}$ & Mo \\
\hline & & \multicolumn{3}{|c|}{$S$} & \multicolumn{3}{|c|}{$\mathrm{Se}$} & \multicolumn{2}{|c|}{$\mathrm{Te}$} \\
\hline \multirow[t]{2}{*}{$\overline{\mathrm{V}}$} & $a_{1}^{\prime}$ & 80.5 & 96.1 & 91.2 & 21.7 & 37.4 & 12.0 & - & - \\
\hline & $e^{\prime}$ & 21.5 & 15.2 & 9.3 & 24.2 & 26.7 & 12.0 & 24.1 & 44.0 \\
\hline \multirow[t]{2}{*}{$\overline{\mathrm{Ti}}$} & $a_{1}^{\prime}$ & 0.00 & 0.00 & 0.00 & 83.3 & 86.5 & 93.8 & - & - \\
\hline & $e^{\prime}$ & 0.00 & 0.00 & 0.00 & 13.3 & 84.8 & 64.4 & 71.4 & 90.2 \\
\hline
\end{tabular}


the delocalized $e^{\prime}$ levels in $\mathrm{MSe}_{2}$ and $\mathrm{MTe}_{2}$ systems lead to weak exchange splitting.

\section{Jahn Teller distortions}

When a metal atom in an $\mathrm{MX}_{2}$ monolayer is substituted with a group IVB or VB element, the difference between the atomic radii of the dopant and host $\mathrm{M}$ atoms leads to a change in the bond length of the dopant and chalcogen atoms. For an $\mathrm{MoS}_{2}$ monolayer doped with $\mathrm{V}$, we saw that its small atomic radius makes the surrounding $\mathrm{S}$ atoms move towards the $\mathrm{V}$ impurity atom, which enhances the hybridization between the $\mathrm{V} d_{3 z^{2}-r^{2}}$ orbital and the $\mathrm{S} p$ orbitals. The relaxation was found to be symmetric, maintaining the local $D_{3 h}$ symmetry with the dopant atom on the high symmetry host site and the chalcogen atoms relaxing towards it (see Fig 3.9). As a consequence, the $a_{1}^{\prime}$ level was lifted (slightly) above the $e^{\prime}$ level as seen in Fig. 4.3. The energy gain from the symmetric relaxation was $\sim 0.15 \mathrm{eV}$. $\mathrm{Nb}$ and Ta displayed a similar behaviour, the important difference being that the chalcogen atoms moved away from them because of their larger atomic radii. Explicit tests found that symmetry-lowering distortions were energetically unfavourable [158].

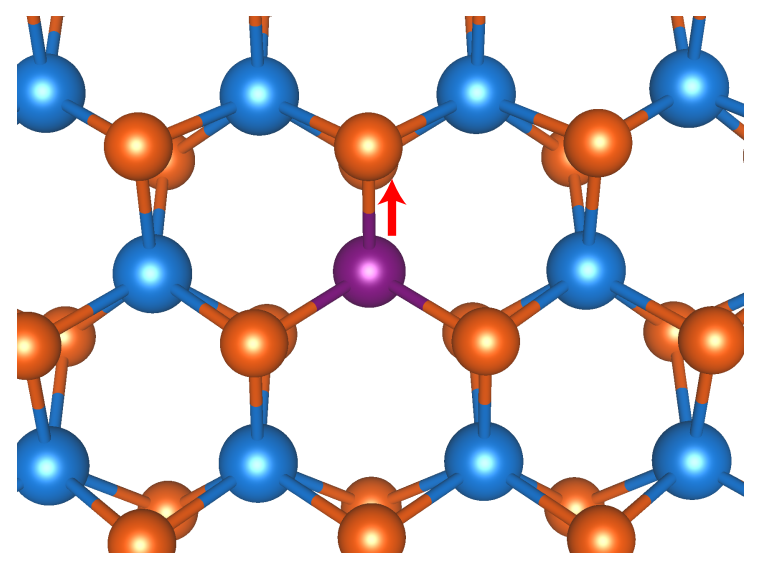

Figure 4.4: (color online). Top view of the dopant-induced Jahn-Teller distortion in an $\mathrm{MX}_{2}$ monolayer. The purple ball is the dopant atom and the red arrow indicates its displacement. Because of the threefold symmetry of the unrelaxed structure, the symmetry-lowering dopant displacement can be in any one of three equivalent directions.

In single-acceptor doped $\mathrm{MoSe}_{2}$ and $\mathrm{MoTe}_{2}$ monolayers, the increased value of $\Delta_{K \Gamma}$ resulting from the lowering of the $\Gamma$ point VBM with respect to the $K / K^{\prime} \mathrm{VBM}$ leads to the $a_{1}^{\prime}$ level lying much lower than the $e^{\prime}$ levels. The hole is accommodated in the doubly degenerate $e^{\prime}$ levels, see Fig. 4.3 and Fig. 4.5, and the system is susceptible to a "Jahn-Teller" (JT) distortion; a symmetry lowering displacement of the dopant atom [177] that lifts the orbital degeneracy. The splitting $\delta$ of the 
only partly filled $e^{\prime}$ level that is linear in the displacement $x$ leads to an energy gain that is also linear in $x$. In equilibrium, where the energy is quadratic in the displacement, this gain of "electronic" energy is balanced by an "elastic" restoring force. As sketched in Fig. 4.4, the displacement of the dopant atom from the center of the trigonal prism $\mathrm{MX}_{6}$ with point group symmetry $D_{3 h}$ lowers the symmetry to $\mathrm{C}_{2 v}$; because the dopant atom moves in the metal plane, mirror symmetry with respect to this metal plane is preserved.

The energy surface of a JT system [178] as a function of the displacement $x$ [177] can be simply modelled as

$$
E_{\mathrm{JT}}(x)=-F_{\mathrm{JT}} x+\frac{1}{2} K x^{2}
$$

where the force driving the JT distortion, $F_{J T}$, is the energy gained by lowering the electronic degeneracy. The elastic restoring force measured by $K$ depends on the strength of the bond between the dopant atom and the neighbouring chalcogen atoms. In equilibrium, $x_{0}=F_{J T} / K$ and $E\left(x_{0}\right)=-F_{J T}^{2} / 2 K$. If the harmonic response of the bonds to the displacement is stiff (large elastic constant $K$ ) and the $e^{\prime}$ orbitals are very delocalized (small $F_{J T}$ ), the JT distortion may be very weak with a negligibly small displacement $x_{0}$.

Although a JT distortion ought to be observed for single-acceptor doped $\mathrm{MoSe}_{2}$ and $\mathrm{MoTe}_{2}$ monolayers, a significant distortion was only found for $\mathrm{V}, \mathrm{Nb}$ and $\mathrm{Ta}$ doped $\mathrm{MoTe}_{2}$ monolayers. For a V-doped $\mathrm{MoTe}_{2}$ monolayer, the large bond length of V-Te indicates a weak elastic restoring force. An elastic constant $K \sim 101 \mathrm{~N} / \mathrm{m}$ is reported [179], which corresponds to $6.3 \mathrm{eV} / \AA^{2}$. The JT displacement is $0.04 \AA$, leading to a splitting of $40 \mathrm{meV}$ for the degenerate $e^{\prime}$ levels (see Fig. 4.3). To estimate the net energy gain of JT displacement, we move the Te atom to the original site and compare its total energy with that of JT displacement. The structure with JT displacement has a net energy gain of $7 \mathrm{meV}$. The elastic energy and electronic energy gain from JT distortion can be estimated to be $5 \mathrm{meV}$ and $12 \mathrm{meV}$, respectively. For the V-doped $\mathrm{MoSe}_{2}$ monolayer, the strong V-Se bond with a significant hybridization of the $\mathrm{V} d_{x y / x^{2}-y^{2}}$ and Se $p$ orbitals leads to a large elastic restoring force with $K \sim 130 \mathrm{~N} / \mathrm{m}\left(8.2 \mathrm{eV} / \AA^{2}\right)$ [179]. On the other hand, the driving force of JT distortion originates from the Coulomb interactions among electrons and nuclei associated with the localization of the degenerate $e^{\prime}$ states. The energy gain from the JT distortion decreases strongly with increasing spatial extent of the wavefunction of $e^{\prime}$ states (effective Bohr radius $a_{0}^{*}$ ) [180]. V-doped $\mathrm{MoSe}_{2}$ has a relative more delocalized $e^{\prime}$ states $\left(a_{0}^{*} \sim 6.9 \AA\right)$ than that in V-doped MoTe 2 monolayer $\left(a_{0}^{*} \sim 5.7\right.$ $\AA$ ), indicating a smaller $F_{J T}$. As a result, the gain of electronic energy from the JT distortion fails to compensate the cost of elastic energy and no JT distortions are found for single-acceptor doped $\mathrm{MoSe}_{2}$ monolayers.

In $\mathrm{CrS}_{2}$ and $\mathrm{WS}_{2}$, a symmetric relaxation appears as in $\mathrm{MoS}_{2}$. For $\mathrm{CrSe}_{2}, \mathrm{CrTe}_{2}$ and $\mathrm{WSe}_{2}$ systems, no JT distortions are found. In general, the large elastic con- 


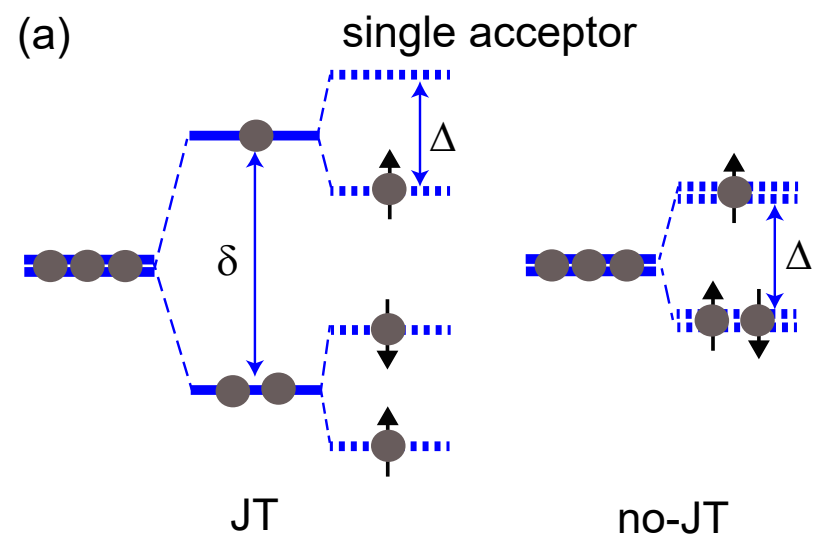

(b) double acceptor
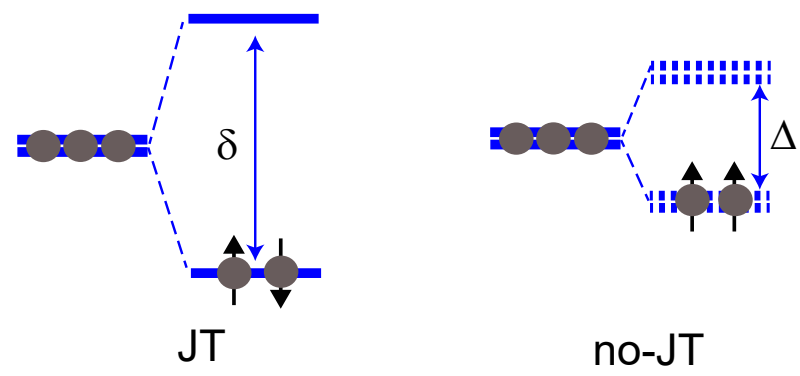

Figure 4.5: (color online). Schematic of the energy gain with and without JahnTeller distortion for (a) single and (b) double acceptors. The solid and dashed blue lines represent spin-degenerate and spin-resolved energy levels, respectively. $\delta$ denotes the energy splitting of the degenerate $e^{\prime}$ levels caused by JT distortion and $\Delta$ is the exchange splitting.

stant and delocalized degenerate orbitals prevent the JT distortion in some systems. For single acceptors, the Jahn Teller distortion does not quench the magnetic moment, Fig. 4.5(a), but the symmetry breaking of the local structure does affect the exchange interaction between dopant pairs. This will be discussed in Sec. 4.4.

For double acceptors, the symmetric relaxation increases the distance between the dopant and nearest neighbour $\mathrm{M}$ atoms, which lowers the $e^{\prime}$ levels far below the $a_{1}^{\prime}$ level in $\mathrm{MS}_{2}$ monolayers (see Fig. 4.3). Two holes occupy the single $a_{1}^{\prime}$ level, resulting in a non-polarized ground state. There is no JT distortion. In $\mathrm{MSe}_{2}$ and $\mathrm{MTe}_{2}$ monolayers, in addition to the symmetric relaxation there is competition between JT distortion and exchange splitting as sketched in Fig. 4.5(b). In the single impurity limit, the double acceptors can be allowed to relax symmetrically with spin polarization in which case they polarize fully (rhs). Or symmetry-lowering distortion can be allowed in which case the JT distortion quenches the magnetic 
moment (lhs). i.e. there are two local minima that are close in energy, a JT state without spin polarization and a spin-polarized state without JT distortion.

Structural relaxations with spin polarization do not break the local symmetry or lead to the dopant atom moving. Except for the position of the dopant atom, the rest atomic position relaxed with spin polarization (spin-polarized state) is almost identical to that relaxed without spin polarization (JT state). We can assume the symmetric relaxation around dopant for these two cases are equal and so the corresponding energy gain. The total energy for these two cases can be represented as

$$
\begin{gathered}
E_{\text {spin }}=E_{0}-E_{\text {symmetric }}-E_{\Delta} \\
E_{J T}\left(x_{0}\right)=E_{0}-E_{\text {symmetric }}-F_{\mathrm{JT}} x_{0}+\frac{1}{2} K x_{0}^{2}
\end{gathered}
$$

in which $E_{\text {symmetric }}$ and $E_{\Delta}$ represent the energy gain from the symmetric relaxation and exchange splitting, respectively; $E_{0}$ denotes the total energy without relaxation or spin polarization, $x_{0}$ denotes the equilibrium displacement of the dopant atom.

The energy gain of the JT state with respect to the spin-polarized state

$$
\Delta E=E_{\text {spin }}-E_{\mathrm{JT}}\left(x_{0}\right)=F_{\mathrm{JT}} x_{0}-\frac{1}{2} K x_{0}^{2}-E_{\Delta} .
$$

In $\mathrm{MSe}_{2}$ and $\mathrm{MTe}_{2}$ monolayers, $\Delta E$ decreases from Ti to Hf, while the exchange splitting $\Delta$ decreases a bit in the same sequence. From Ti to Hf, the impurity states become more shallow and delocalized, which leads to a weaker JT force $F_{\mathrm{JT}}$ and

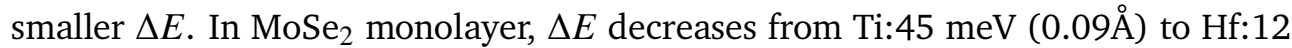
$\mathrm{meV}\left(0.14 \AA\right.$ ) . As discussed above, $\mathrm{MoTe}_{2}$ monolayer has a relative small $K$ and large $F_{\text {JT }}$ with respect to $\mathrm{MoSe}_{2}$ monolayer. As a result, $\Delta E$ is quiet large for $\mathrm{MoTe}_{2}$

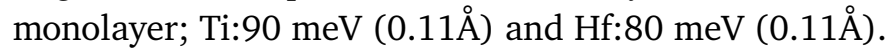

In $\mathrm{CrSe}_{2}$ and $\mathrm{CrTe}_{2}$, the shallow $e^{\prime}$ states (see Fig. 4.3) are pretty delocalized, which indicates a very weak JT driving force. The $a_{1}^{\prime}$ levels that are close to the $e^{\prime}$ levels accommodate part of the holes. This increases the $E_{\Delta}$ for the large exchange splitting of the $a_{1}^{\prime}$ levels. For Ti-doped $\mathrm{CrSe}_{2}$ and $\mathrm{CrTe}_{2}$ monolayers, the states with spin polarization gains more energy than the JT state, about $54 \mathrm{meV}$ and $42 \mathrm{meV}$, respectively. $\mathrm{WSe}_{2}$ has a large elastic constant $\mathrm{K} \sim 147 \mathrm{~N} / \mathrm{m}[179]\left(9.2 \mathrm{eV} / \AA^{2}\right) . \Delta E$ are pretty small; $10 \mathrm{meV}(0.14 \AA)$ for Ti and $5 \mathrm{meV}(0.12 \AA)$ for Hf.

\subsubsection{Single ion anisotropy}

In the isotropic 2D Heisenberg model with a gapless spin-wave spectrum, thermal fluctuations at any finite temperature will destroy long-range magnetic ordering while the magnetic anisotropy implicit in the 2D Ising model makes the system undergo a phase transition at a finite temperature to a magnetically ordered state [63; 64]; magnetic anisotropy is essential for long-range ferromagnetic ordering 


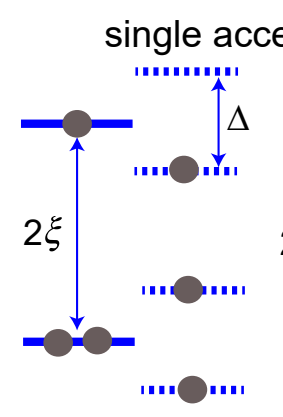

$\mathrm{M} / / \mathrm{c}$

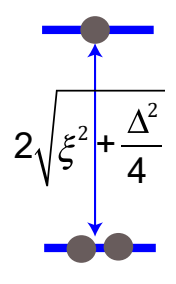

$\mathrm{M} / \mathrm{a}$

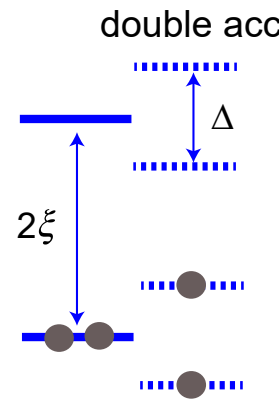

$\mathrm{M} / / \mathrm{c}$

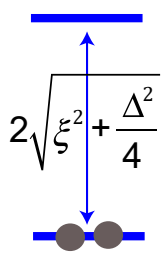

$\mathrm{M} / / \mathrm{a}$

Figure 4.6: (color online). Schematic diagram of the energy gain with spin orientation in-plane $(\mathrm{M} / / \mathrm{a})$ and out of plane $(\mathrm{M} / / \mathrm{c})$ for single and double acceptors. The solid and dashed blue lines represent spin degenerate and spin resolved levels, respectively.

in two dimensions. Though not constraining the direction of the spin as strictly as in the Ising model, magnetocrystalline anisotropy from exchange interactions or single ion anisotropy (SIA) may lead to an "easy axis" for the orientation of the spins to reduce their energy. In the recently reported ferromagnetic $\mathrm{CrI}_{3}$ and $\mathrm{Fe}_{2} \mathrm{GeTe}_{3}$ monolayers, single ion anisotropy plays an important role in stabilizing the longrange order $[141 ; 181]$. We can expect the same to hold for $\mathrm{MX}_{2}$ monolayers doped to make them magnetic making it important to determine their magnetic anisotropy. We will study this in the single impurity limit where it is called the SIA.

First let us consider the low energy Hamiltonian of the doped system including spin orbit coupling (SOC) and spin polarization

$$
H=H_{0}+\Delta \mathbf{m} \cdot \mathbf{s}+\xi \mathbf{l} \cdot \mathbf{s}
$$

in which $H_{0}$ is the spin-independent part of the Hamiltonian, $\Delta \mathbf{m}$ is the exchange field that leads to an exchange splitting $\Delta, \mathbf{m}$ is a unit vector in the direction of the magnetization, $\mathbf{m} \equiv \mathbf{M} /|\mathbf{M}|$, and $\xi$ is the conventional spin-orbit coupling parameter. In the subspace of the $l=2, m_{l}= \pm 2$ orbitals

$$
\xi \mathbf{l} \cdot \mathbf{s}=\frac{\xi}{2}\left(\begin{array}{cc}
l_{z} & l_{-} \\
l_{+} & -l_{z}
\end{array}\right)=\left(\begin{array}{cc}
\xi & 0 \\
0 & -\xi
\end{array}\right)
$$

where we use Hartree atomic units with $\hbar=1$. For $\mathbf{M} \| c$,

$$
\Delta \mathbf{m} \cdot \mathbf{s}=\left(\begin{array}{cc}
\frac{\Delta}{2} & 0 \\
0 & -\frac{\Delta}{2}
\end{array}\right) .
$$


and the SOC Hamiltonian can be written as

$$
H=H_{0}+\left(\begin{array}{cccc}
\xi+\frac{\Delta}{2} & 0 & 0 & 0 \\
0 & -\xi-\frac{\Delta}{2} & 0 & 0 \\
0 & 0 & -\xi+\frac{\Delta}{2} & 0 \\
0 & 0 & 0 & \xi-\frac{\Delta}{2}
\end{array}\right)
$$

Rotating the spin quantization direction from $c$ to $a$ we get for $\mathbf{M} \| a$,

$$
H=H_{0}+\left(\begin{array}{cccc}
\xi & \frac{\Delta}{2} & 0 & 0 \\
\frac{\Delta}{2} & -\xi & 0 & 0 \\
0 & 0 & -\xi & \frac{\Delta}{2} \\
0 & 0 & \frac{\Delta}{2} & \xi
\end{array}\right)
$$

Then we can estimate the single ion anisotropy to be

$$
E_{\mathrm{MAE}}= \begin{cases}\xi+\frac{\Delta}{2}-\sqrt{\xi^{2}+\frac{\Delta^{2}}{4}} & \text { for } \mathrm{V}, \mathrm{Nb}, \mathrm{Ta} \\ 2 \xi-\sqrt{4 \xi^{2}+\Delta^{2}} & \text { for Ti, Zr, Hf }\end{cases}
$$

where positive values favour an out-of-plane spin orientation and negative values in-plane. Equation (4.11) shows how the SIA depends on both the SOC and exchange interaction. It is clear that single acceptors give positive values, while double acceptors give negative values if the magnetic moment is not quenched by a JT distortion.

We calculated the SIA numerically from first principles using the force theorem $[132 ; 133]$ which expresses the difference between two total energies as the difference in the sum of Kohn-Sham eigenvalues [16] for $\mathbf{M} \| a$ and $\mathbf{M} \| c[134 ; 139 ; 136]$. Table 4.5 compares the SIA from (4.11) with the first principles results. We note that the SOC for the $e^{\prime}$ levels of the V-doped $\mathrm{MoS}_{2}$ monolayer is similar (130 meV) to that for the $K / K^{\prime}$ valley of pristine $\mathrm{MoS}_{2}$ monolayer, while the $a_{1}^{\prime}$ level has no SOC just as the $\Gamma$ valley of the $\mathrm{MoS}_{2}$ monolayer has none. The exchange splitting is $15.2 \mathrm{meV}$ and $96 \mathrm{meV}$ for the $e^{\prime}$ and $a_{1}^{\prime}$ levels, respectively. In Table 4.5, we see an estimate of $7.2 \mathrm{meV}$ using (4.11), larger than what we calculate from first principles, $4.5 \mathrm{meV}$. We note that the $a_{1}^{\prime}$ level may accommodate some of the hole but does not contribute to the SIA leading to the first-principles SIA being smaller than that estimated using (4.11) that does not take this factor into consideration. In addition, because a finite size supercell $(12 \times 12)$ was used in the first-principles calculation, the $e^{\prime}$ level has become a band with a finite dispersion and this will reduce the energy gain from the exchange splitting $\Delta$ and therefore also reduce the SIA. The values from (4.11) are a simple estimate of the SIA in the single impurity limit with all holes in $e^{\prime}$ states. For all of the systems in Table 4.5 there is reasonable 
Table 4.5: Calculated spin orbit splitting, exchange splitting, and SIA in meV for the degenerate $e^{\prime}$ levels in monolayers of $\mathrm{MX}_{2}(12 \times 12$ supercell $)$ doped with $\mathrm{V}$ and Ti substituting a single Mo. The SIA estimated from (4.11) is also shown for comparison. The atomic structures are fully relaxed with spin polarization.

\begin{tabular}{|c|c|c|c|c|c|c|c|c|c|}
\hline \multirow{3}{*}{$\begin{array}{l}\mathrm{M} \\
\mathrm{X}\end{array}$} & & $\mathrm{Cr}$ & Mo & $\mathrm{W}$ & $\mathrm{Cr}$ & Mo & $\mathrm{W}$ & $\mathrm{Cr}$ & Mo \\
\hline & & \multicolumn{3}{|c|}{ S } & \multicolumn{3}{|c|}{ Se } & \multicolumn{2}{|c|}{$\mathrm{Te}$} \\
\hline & $2 \xi$ & 66 & 130 & 187 & 78 & 107 & 181 & 91 & 110 \\
\hline \multirow{3}{*}{ V } & $\Delta$ & 21.5 & 15.2 & 9.3 & 24.2 & 26.7 & 12.0 & 24.1 & 44.0 \\
\hline & SIA: (4.11) & 9.0 & 7.2 & 4.5 & 10.3 & 11.0 & 5.8 & 10.5 & 17.8 \\
\hline & SIA:DFT & 4.7 & 4.5 & 3.2 & 7.2 & 10.0 & 4.7 & 7.7 & 11.2 \\
\hline \multirow[t]{4}{*}{$\mathrm{Ti}$} & $2 \xi$ & 0.0 & 0.0 & 0.0 & 78 & 87 & 148 & 83 & 105 \\
\hline & $\Delta$ & 0.0 & 0.0 & 0.0 & 13.3 & 84.8 & 64.4 & 71.4 & 90.2 \\
\hline & SIA: (4.11) & 0.0 & 0.0 & 0.0 & -1.1 & -34.4 & -13.4 & -26.4 & -31.1 \\
\hline & SIA:DFT & 0.0 & 0.0 & 0.0 & -0.3 & -24.1 & -5.2 & -23.4 & -25.5 \\
\hline
\end{tabular}

qualitative agreement between the DFT supercell calculations and the SIA estimate of equation (4.11).

In the limit $\Delta \ll \xi, E_{\text {SIA }} \sim \Delta\left(1-\frac{\Delta}{2 \xi}\right)$ for single acceptors and the SIA depends mainly on the exchange splitting $\Delta$. In $\mathrm{MoSe}_{2}$, the SIA can be as high as $10 \mathrm{meV} /$ dopant, larger than the $4.5 \mathrm{meV} /$ dopant in $\mathrm{MoS}_{2} . \mathrm{V}$ in $\mathrm{MoTe}_{2}$ has a very large exchange splitting, but because of the JT distortion the SIA is not as high as the value estimated from (4.11). Pristine $\mathrm{WS}_{2}$ and $\mathrm{WSe}_{2}$ monolayers have a large spin-orbit splitting of the $K / K^{\prime}$ valley $(\sim 0.4-0.6 \mathrm{eV})$, but the splitting of the impurity levels in these systems is only half of these values. For the delocalized holes, $\Delta$ is small and determines the SIA. The impurity states have a large $\Delta$ in $\mathrm{CrX}_{2}$ monolayers, but the small SOC leads to SIA values between those for MoX and $\mathrm{WX}_{2}$.

Table 4.5 shows that the exchange splitting can become very large for double acceptors because of the increased hole density and the SIA can be as large as $25.5 \mathrm{meV}$ in magnitude for Ti doped $\mathrm{MoTe}_{2}$. The negative values favour in-plane magnetization to be described by an XY spin model. In the 2D XY spin model, there is no conventional transition to long-range magnetic ordering. As discussed in Sec. 4.3.2 for double acceptors, there is competition between JT distortion and exchange splitting and the JT distortion will quench the magnetic moment if it is sufficiently strong. Doping $\mathrm{MX}_{2}$ monolayers with double acceptors is therefore not a viable route to realizing ferromagnetic semiconductors.

When the doping concentration is increased, the MAE decreases because of the reduced exchange splitting. For $100 \% \mathrm{~V}$ doping ( $\mathrm{VX}_{2}$ monolayers), we calculate an MAE of $\sim-0.2 \mathrm{meV} /$ atom from first principles with the spin rotating from the $\mathrm{Z}$ axis to the $\mathrm{XY}$ plane. This may explain the inconsistent experimental results on 
Table 4.6: Calculated formation energy $(\mathrm{eV})$ of a chalcogen vacancy $\varnothing_{\mathrm{X}}$, a transition metal vacancy $\varnothing_{\mathrm{M}}$, and an $\mathrm{M}_{\mathrm{X}}$ antisite (one transition metal atom occupying a chalcogen atom site) in $\mathrm{MX}_{2}$ monolayer with relaxation.

\begin{tabular}{ccccccccc}
\hline & $\mathrm{CrS}_{2}$ & $\mathrm{MoS}_{2}$ & $\mathrm{WS}_{2}$ & $\mathrm{CrSe}_{2}$ & $\mathrm{MoSe}_{2}$ & $\mathrm{WSe}_{2}$ & $\mathrm{CrTe}_{2}$ & $\mathrm{MoTe}_{2}$ \\
\hline$\varnothing_{\mathrm{X}}$ & 2.47 & 3.15 & 3.01 & 2.46 & 2.77 & 2.72 & 2.26 & 2.89 \\
$\varnothing_{\mathrm{M}}$ & 6.31 & 7.87 & 7.27 & 4.80 & 6.04 & 5.85 & 2.44 & 4.60 \\
$\mathrm{M}_{\mathrm{X}}$ & 4.72 & 6.71 & 7.12 & 4.44 & 5.51 & 6.48 & 3.31 & 4.61 \\
\hline
\end{tabular}

the ferromagnetism of $\mathrm{VSe}_{2}$ monolayers $[75 ; 182]$ where a charge density wave is normally blamed for destroying the ferromagnetic ordering. Recently reported $\mathrm{VSe}_{2} / \mathrm{Co}$ and $\mathrm{VSe}_{2} / \mathrm{Fe}$ heterostructures have a magnetic transition at finite temperature $[183 ; 184]$. This might be related to the interface anisotropy or hybridization between $\mathrm{V}$ d orbitals and Co or Fe d orbitals, which leads to an out-of-plane anisotropy in $\mathrm{VSe}_{2}$ monolayer and opens a gap in the spin-wave spectrum of the system.

\subsubsection{Interaction with intrinsic defects}

Intrinsic defects like vacancies and antisite defects are ubiquitous in van der Waals materials and have been investigated in much experimental $[83 ; 185 ; 186]$ and computational $[187 ; 84 ; 188]$ work. We denote $\mathrm{M}$ and $\mathrm{X}$ vacancies as $\varnothing_{\mathrm{M}}$ and $\varnothing_{\mathrm{X}}$, respectively. Similarly, antisite defects are denoted $A_{B}$ for an $A$ atom on a $B$ site; thus in the present case we will be concerned with $M_{X}$. We will focus on the magnetism introduced or affected by these intrinsic defects in $p$-doped systems. The formation energies of various intrinsic defects are listed in Table 4.6. The formation energy $E\left[\varnothing_{X}\right]$ of a chalcogen X atom vacancy is about half that of a transition metal vacancy, $E\left[\varnothing_{\mathrm{M}}\right]$, or half that of an antisite defect with a metal atom on an X site, $E\left[\mathrm{M}_{\mathrm{X}}\right]$, indicating that chalcogen vacancies are more likely to be formed in thermodynamic equilibrium than the other two defects. The formation energies $\varnothing_{\mathrm{M}}$ and $\mathrm{M}_{\mathrm{X}}$ are close and decrease from $\mathrm{MS}_{2}$ to $\mathrm{MTe}_{2}$. Compared to the formation energy of acceptors (see Table 4.2), much more energy is required for the formation of intrinsic defects indicating that acceptors may be incorporated into $\mathrm{MX}_{2}$ monolayers at low temperature to suppress the formation of intrinsic defects.

As in the case of substitutionally doped $\mathrm{MX}_{2}$ monolayers, Fig. 4.7(e), defect states associated with intrinsic defects can appear in the band gap and interplay with those introduced by $p$ doping. Taking an $\mathrm{MoS}_{2}$ monolayer as prototype, we plot the non spin-polarized band structures for a number of intrinsic defects and intrinsic defects combined with $\mathrm{V}$ dopants, $\mathrm{V}_{\mathrm{Mo}}$. For simplicity, the structures are not relaxed and the effect of relaxation is discussed in the text.

A Mo vacancy $\varnothing_{M}$ with a formation energy of $7.87 \mathrm{eV}$ introduces five mid-gap 

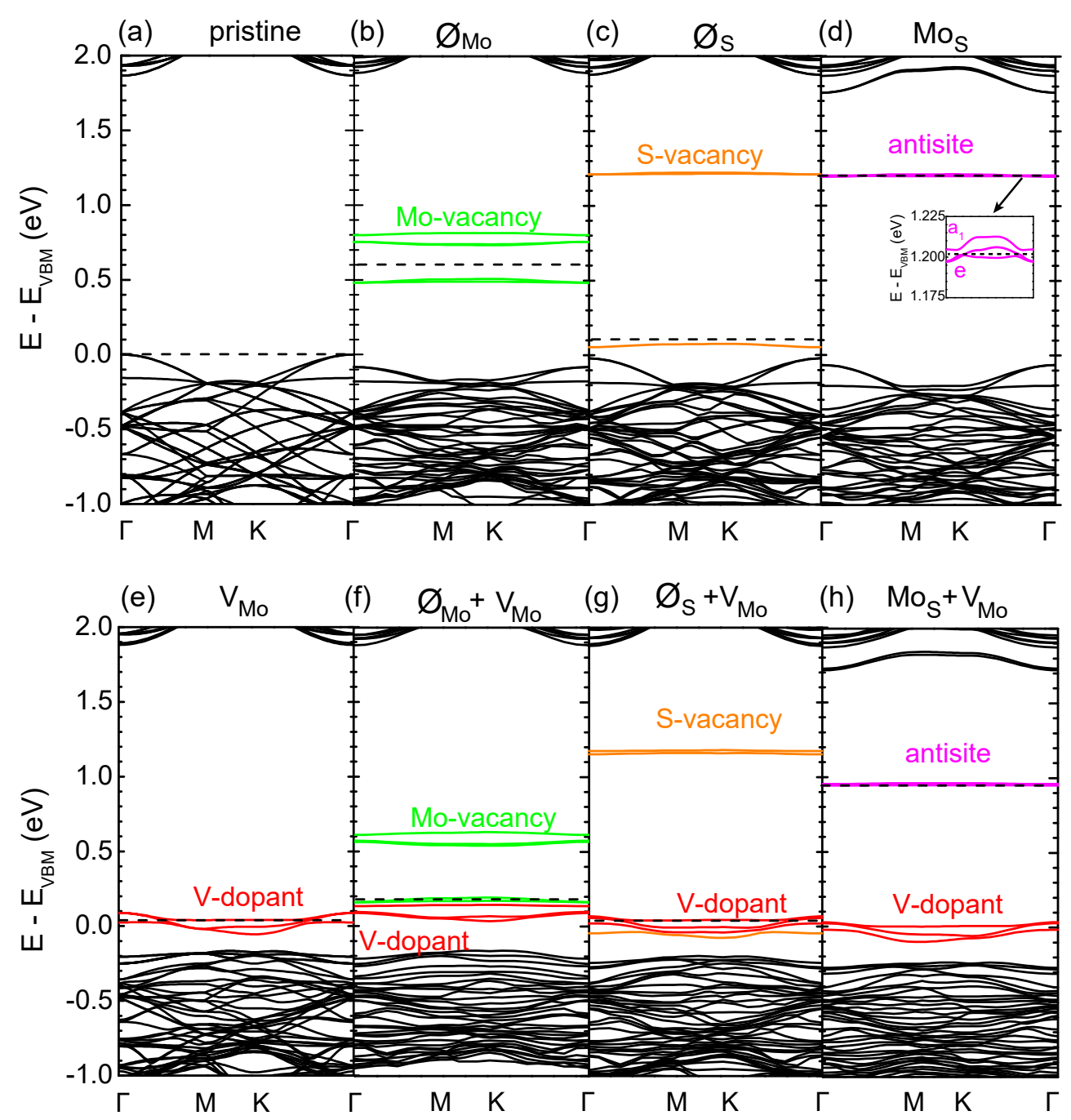

Figure 4.7: (color online). Band structures for (a) a defect-free $\mathrm{MoS}_{2}$ monolayer, (b) a vacancy on a Mo site, $\varnothing_{\mathrm{Mo}}$, (c) a vacancy on a sulfur site, $\varnothing_{\mathrm{Mo}}$, (d) a Mos antisite defect consisting of a Mo atom on a S site, (e) a vanadium atom on a Mo site, $\mathrm{V}_{\text {Mo, }}$ (f) two neighbouring Mo sites, one occupied by a substitutional vanadium atom, the other with a Mo vacancy, $\varnothing_{M_{0}}+V_{M o}(g)$ a sulfur vacancy with a vanadium atom on one of the neighbouring Mo sites, $\varnothing_{\mathrm{Mo}}+\mathrm{V}_{\mathrm{Mo}}$, (h) a Mos antisite defect with a vanadium atom on one of the neighbouring Mo sites, $\mathrm{Mo}_{\mathrm{S}}+\mathrm{V}_{\mathrm{Mo}}$. All modelled for $\mathrm{MoS}_{2}$ with a $6 \times 6$ supercell. The electronic structures are aligned on the top of the valence band and the Fermi level is indicated by a dashed black line. The inset in (d) is zoom in band structure near the Fermi level. 
states, one singly degenerate state and two doubly degenerate states under local $D_{3 h}$ symmetry as shown in Fig. 4.7(b). Six holes are hereby introduced into the system that occupy the three defect states above the Fermi level. Structural relaxation preserves the $D_{3 h}$ symmetry as expected from the absence of partially occupied orbitally degenerate states. However, the relative position of the deep defect states depends on the particular system under consideration. For example, in a $\mathrm{MoTe}_{2}$ monolayer with a Mo vacancy, the singly degenerate state goes far below the doubly degenerate states and the Fermi level lies in the doubly degenerate states, which leads to a JT distortion.

A sulfur vacancy, $\varnothing_{S}$, with a formation energy of $3.15 \mathrm{eV}$, introduces three gap states with an orbitally doubly degenerate unoccupied state in the middle of the gap and a single occupied state close to the VBM, Fig. 4.7(c). Usually a sulfur vacancy is considered to be an electron donor but for that to be true, the occupied state would have to be close to the CBM which is not the case. There are no partially filled defect states so that structural relaxation preserves the $C_{3 v}$ site symmetry. The relaxation makes the Mo atoms surrounding the $S$ vacancy come close, which lifts a bit the occupied singly degenerate $S$ vacancy defect state. This is true for all $\mathrm{MX}_{2}$ monolayers and chalcogen vacancies do not introduce magnetism or induce JT distortions.

A Mos antisite consists of a sulfur vacancy occupied by an extra Mo atom. The $\mathrm{S}$ vacancy removes two holes while the Mo atom contributes six electrons, Fig. 4.7(d). Four of these electrons are accommodated in the sulfur vacancy mid-gap states that are pulled down into the valence band by the attractive Mo potential leaving two electrons in a deep doubly degenerate $e$ state under the local $C_{3 v}$ symmetry that is quasi degenerate with an $a_{1}$ singlet and giving rise to a magnetic moment of $2 \mu_{B}$. The inset of Fig. 4.7(d) shows a zoom-in of the band structure near the Fermi level. For the partially occupied degenerate $e$ levels, JT distortion could lift the degeneracy but is not strong enough to do so and spin polarization prevails. For the much deep antisite states, the doubly degenerate states have an exchange splitting of 0.33 $\mathrm{eV}$. In a $\mathrm{MoTe}_{2}$ monolayer, relaxation lowers the $a_{1}$ level well below the $e$ levels. The $a_{1}$ level accommodates both electrons and a $\mathrm{Mo}_{\mathrm{Te}}$ antisite is nonmagnetic.

As discussed at length in Chapter 3 , a vanadium atom on a Mo site, $\mathrm{V}_{\mathrm{Mo}}$, induces three shallow impurity like levels above the VBM which are shown unrelaxed in Fig. 4.7(e). With relaxation, we see these levels with binding energies of about $60 \mathrm{meV}$ in Fig. 4.3. The intrinsic defect states shown in Fig. 4.7(b-d) have very deep gap states $(>0.5 \mathrm{eV})$, indicating that the defect potential is effectively more attractive for holes than the substitutional $\mathrm{V}$ dopant. If we introduce a Mo vacancy next to a $\mathrm{V}$ dopant $\left(\varnothing_{\mathrm{Mo}}+\mathrm{V}_{\mathrm{Mo}}\right)$, the $\mathrm{V}_{\mathrm{Mo}}$ hole will transfer to the $\mathrm{S}$ atoms surrounding the Mo vacancy. As shown in Fig. 4.7(f), the hole goes into the lowest lying doubly degenerate $\varnothing_{\text {Mo }}$ defect state and polarizes fully; this might suppress the quenching of the magnetic moment we found for close $\mathrm{V}$ dopant pairs.

A sulfur vacancy, $\varnothing_{S}$, has one fully occupied defect state that is very close to the 
VBM, Fig. 4.7(c). For neighbouring $\varnothing_{\mathrm{S}}+\mathrm{V}_{\mathrm{Mo}}$ defects, the shallow quasidegenerate defect states hybridize leading to a spatial redistribution of the hole, see Fig. 4.7(g). It should be noted that the defect states of $\varnothing_{\mathrm{S}}$ mainly consist of Mo $d$ states on the three Mo atoms that surround the $\mathrm{S}$ vacancy. The hybridization between $\mathrm{V}$ and Mo $d$ orbitals enhances the magnetism as evidenced by an increased exchange splitting. Under relaxation, the Mo atoms surrounding the $S$ vacancy move towards the $\mathrm{V}$ dopant atom, which further enhances the hybridization of the $\mathrm{V}$ dopant states with $S$ vacancy states and so the magnetism.

For a vanadium atom on a Mo site next to a Mo atom on a S site, a $\mathrm{Mo}_{S}+\mathrm{V}_{\mathrm{Mo}}$ defect pair, the hole introduced by $\mathrm{V}_{\mathrm{Mo}}$ moves to the partially filled Mos mid-gap states, resulting in a net magnetic moment of $1 \mu_{B}$ on the antisite Mo atom. the $\mathrm{V}$ dopant atom gets one extra electron from the antisite that quenches the magnetic moment on the dopant atom. With relaxation, a JT distortion appears for the partially filled degenerate defect states of the antisite and the system keeps spin polarized.

In general, all of the intrinsic defects we have examined are quite localized. Even though the magnetization can be enhanced by the hybridization between intrinsic defects and dopant states in the sense that the exchange splitting is increased, this is unlikely to extend the range of the exchange interaction between magnetic defects. It has been reported that the existence of $S$ vacancies might reduce the barrier for dopant diffusion [189], which can decrease the percolation threshold. This could increase the Curie temperature at low doping concentration, where dopant can form pairs in the presence of $S$ vacancies.

\subsubsection{Electron doping: Rhenium}

So far we have only considered the case of hole doping. Here we briefly consider the case of electron doping by the $5 d$ element rhenium that gives rise to an attractive Coulomb potential in the single impurity limit. We expect the electron-doping case to be more complex because according to Fig. 4.1 the conduction band minima (CBM) at $K / K^{\prime}$ will give rise to two degenerate states while the six $Q$ point minima between $K$ and $\Gamma$ will give rise to an additional six degenerate states that will be coupled in degenerate perturbation theory by the Coulomb potential in the effective mass approximation. The intervalley scattering between the $K$ and $K^{\prime}$ valleys and the sixfold $Q$ valleys leaves a single $a_{1}^{\prime}$ level with $d_{3 z^{2}-r^{2}}$ character and a doubly degenerate $e^{\prime}$ level with mixed $\left\{d_{x^{2}-y^{2}}, d_{x y}\right\}$ character below the CBM as indicated in Fig. 4.8 with a binding energy of $92 \mathrm{meV}$. The lowest lying $a_{1}^{\prime}$ state polarizes fully in the single impurity limit. In spite of its $d_{3 z^{2}-r^{2}}$ character, the exchange splitting is weak ( $46 \mathrm{meV}$ ). This can also be seen from the large dispersion of the $a_{1}^{\prime}$ level shown in Fig. 4.8. The small SOC in the conduction band leads to a negligible SIA. As we found for pairs of substitutional V atoms in a $\mathrm{MoS}_{2}$ monolayer in Chapter 3, below a critical separation the magnetic moment is quenched by a strong $\pi$ bond 


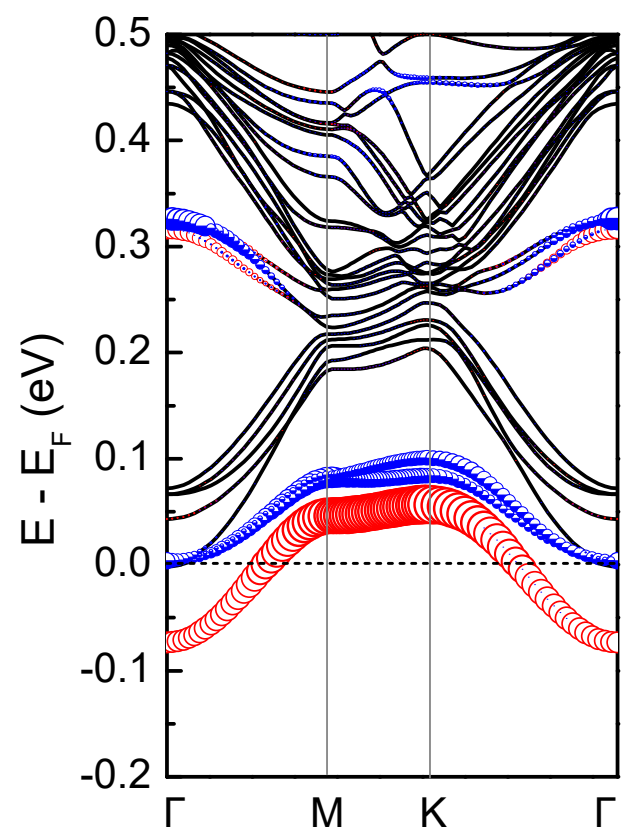

Figure 4.8: (color online). The orbital resolved band structure of $\mathrm{MoS}_{2}$ monolayer with one Re substitutionally doping $(6 \times 6$ supercell) at Mo site. The open red circles and half-filled blue circles represent the contribution from $\operatorname{Re} d_{3 z^{2}-r^{2}}$ and $d_{x^{2}-y^{2}}, d_{x y}$, respectively. The Fermi level is indicated by black dashed line.

being formed between Re pairs.

\subsection{Discussion}

The behaviour of substitutional dopants in TMD monolayers has its origin in the electronic properties of the host. In $\mathrm{MoS}_{2}$ and $\mathrm{WS}_{2}$ monolayers, the small energy difference $\Delta_{K \Gamma}$ between the $K / K^{\prime}$ and $\Gamma$ maxima leads to the hole occupying the $a_{1}^{\prime}$ level from the $\Gamma$ valley. For pairs of dopant atoms that are sufficiently close, the magnetic moment is quenched by the formation of singlet bonding states. This can be avoided by increasing $\Delta_{K \Gamma}$ as happens for $\mathrm{MSe}_{2}$ and $\mathrm{MTe}_{2}(\mathrm{X}=\mathrm{Cr}, \mathrm{Mo}, \mathrm{W}$, see Table 4.1). Even the slightly larger value of $\Delta_{K \Gamma}$ for $\mathrm{CrS}_{2}$ is enough to suppress the quenching. Nevertheless, in order to realize long range and strong exchange interactions at high temperature, the impurity states should have large effective Bohr radii as well as large exchange splitting. In addition, the SIA of single acceptors also requires a large exchange splitting, while double acceptors are excluded because of their in-plane SIA.

Comparing the exchange splitting parameter $\Delta$ for $\mathrm{V}$-doped $\mathrm{MX}_{2}$ monolayers 
in Table 4.5, p-doped $\mathrm{CrS}_{2}, \mathrm{CrSe}_{2}, \mathrm{CrTe}_{2}, \mathrm{MoSe}_{2}$, and $\mathrm{MoTe}_{2}$ are seen to have reasonably large values of $\Delta$ while WSe $e_{2}$ can be excluded because its $\Delta$ is so small. The JT distortion will lift the degeneracy of the partially filled $e^{\prime}$ levels in $\mathrm{MoTe}_{2}$ monolayers making this system prefer singlet states. The effective Bohr radii for Vdoped $\mathrm{CrS}_{2}, \mathrm{CrSe}_{2}, \mathrm{CrTe}_{2}$, and $\mathrm{MoSe}_{2}$ are $8.3 \AA$, 8.9 $9.9 .3 \AA$, and 6.9 $\mathrm{A}$, respectively, indicating the expected range of the exchange interaction.

As in Chapter 3, we can map the exchange interactions onto a Heisenberg model and calculate the total energy difference between ferromagnetic and antiferromagnetic states for $\mathrm{V}$ pairs. These energy differences are plotted in Fig. 4.9 and Fig. 4.10. Even though the Bohr radii of $e^{\prime}$ levels in V-doped $\mathrm{CrS}_{2}$ is $8.3 \AA$, the exchange interaction drops faster with dopant separation than in $\mathrm{MoSe}_{2}$ which has a smaller effective Bohr radius. This is because the $a_{1}^{\prime}$ level in V-doped $\mathrm{CrS}_{2}$ is so close to the $e^{\prime}$ levels (see Fig. 4.3) that are partially polarized, as we originally found for V-doped $\mathrm{MoS}_{2}$. The difference is that the $a_{1}^{\prime}$ level is not high enough to quench the magnetic moment for even the closest separation. It should be noted that in $\mathrm{CrS}_{2}, \mathrm{CrSe}_{2}, \mathrm{CrTe}_{2}$ and $\mathrm{MoSe}_{2}$ the JT distortions are negligible. So the doubly degenerate impurity levels remain degenerate and the exchange interactions do not change much with relaxation. According to Fig. 4.9, V-doped $\mathrm{CrTe}_{2}$ exhibits the longest range exchange interaction of all the systems we have studied. We use Monte Carlo calculations to estimate the Curie temperature $\left(T_{C}\right)$ and plot it for

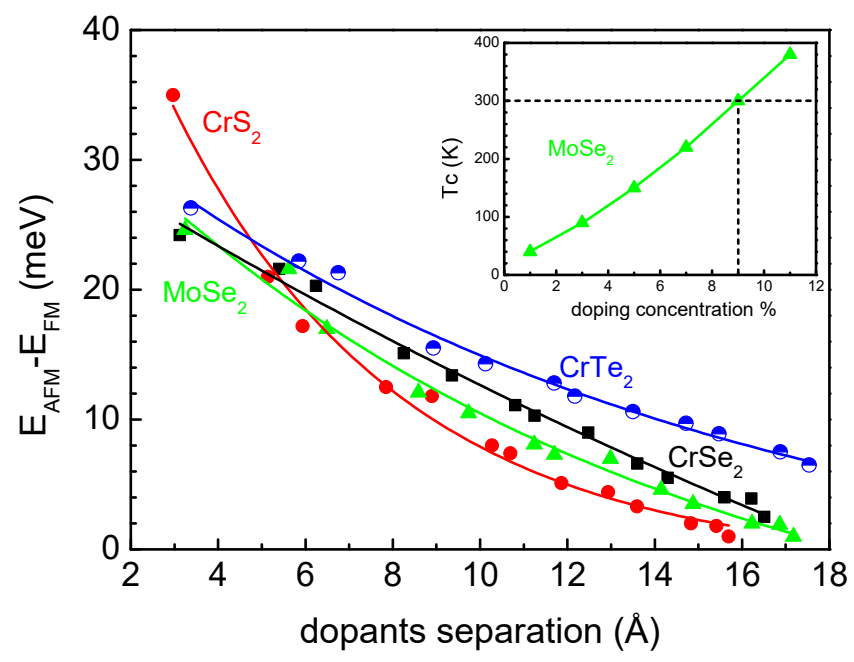

Figure 4.9: (color online). The difference between the total energies of antiferromagnetic and ferromagnetic states for pairs of $\mathrm{V}$ substitutional impurities plotted as a function of their separation for $\mathrm{MX}_{2}(\mathrm{M}=\mathrm{Cr}, \mathrm{Mo} ; \mathrm{X}=\mathrm{S}, \mathrm{Se})$ monolayer in relaxed $12 \times 12$ supercell. The inset plots the Curie temperature as a function of doping concentration for V-doped $\mathrm{MoSe}_{2}$. The solid and dash lines are a guide to the eyes. 


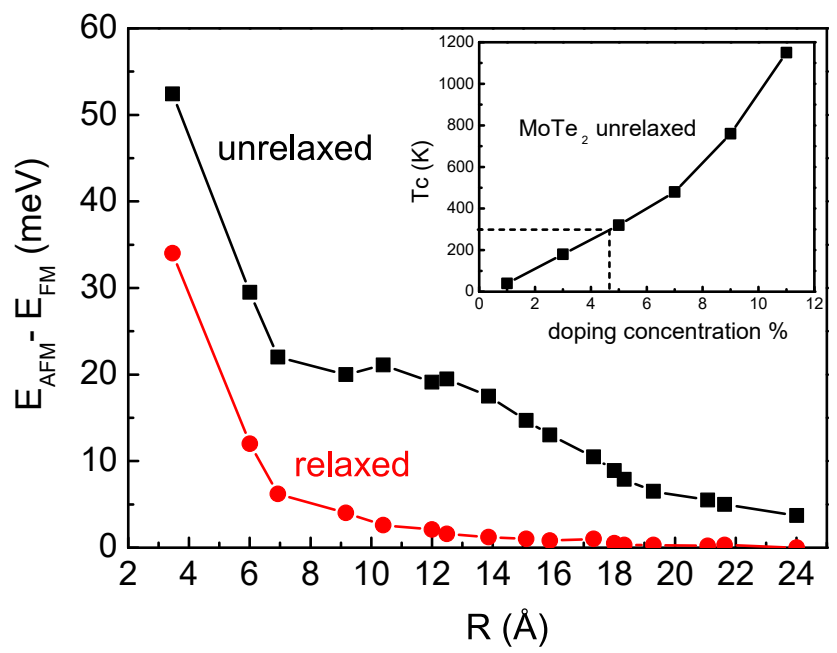

Figure 4.10: (color online). The same as Fig. 4.9 but for V-doped $\mathrm{MoTe}_{2}$ monolayer with (red dots) and without (black squares) relaxation. The inset plots the Curie temperature as a function of doping concentration for unrelaxed V-doped $\mathrm{MoTe}_{2}$.

V-doped $\mathrm{MoSe}_{2}$ as a function of vanadium concentration in the inset to Fig. 4.9. For a 9\% concentration of $\mathrm{V}$ dopant, a room temperature value of $T_{C}$ is predicted.

Of all the TMD monolayers we have considered, V-doped $\mathrm{MoTe}_{2}$ has the largest exchange splitting. Before relaxation the $\mathrm{V}$ pairs show a large and long-range exchange FM coupling with very high Curie temperatures as shown in the inset to Fig. 4.10. However, when relaxation is considered, the JT distortion splits the degenerate levels and leads to a reduced MAE as we found in Sec. 4.3.2. The exchange interaction then decreases very significantly with dopant separation, see Fig. 4.10. One possibility to circumvent this would be to consider doping a $\operatorname{Mo}\left(\operatorname{Se}_{x} \operatorname{Te}_{1-x}\right)_{2}$ substitutional alloy with $\mathrm{V}$.

Monte Carlo calculations show that $9 \%$ of $\mathrm{V}$ doped in $\mathrm{MoSe}_{2}$ monolayer can have Curie temperature as high as 300K. For itinerant electrons occupying narrow bands, it has been argued that the effective interaction predicted by the Stoner criterion will not be reduced by correlation effects or spin wave excitations [108]. The completely spin polarized carriers in narrow impurity bands lead to a large spin stiffness and develop ferromagnetic ordering by their mutual interaction.

\subsection{Conclusions}

We have used $a b$ initio calculations to systematically investigate the structural and electronic properties of $\mathrm{MX}_{2}$ monolayers doped with group IVB and VB transition metals. Three impurity levels are identified above the VBM that determine the 
structural and electronic properties of the doped systems. To avoid the magnetic moment quenching by singlet formation for close dopant pairs, the holes should reside on the degenerate $e^{\prime}$ levels. This also gives rise to large single ion anisotropies for the strong spin-orbit coupling in $\mathrm{MX}_{2}$ monolayers. The partially occupied degenerate levels might, however, lead to Jahn-Teller distortions and quenching of the magnetic moments. Double acceptors have an in-plane single ion anisotropy and can not develop long-range ordering. Intrinsic defects may localize the holes and enhance the exchange splittings in $p$-doped $\mathrm{MX}_{2}$ monolayers. Considering the exchange splitting and effective Bohr radii of $\mathrm{V}, \mathrm{Nb}$ and Ta dopants, four candidates $\left(\mathrm{CrS}_{2}, \mathrm{CrSe}_{2}, \mathrm{CrTe}_{2}\right.$, and $\mathrm{MoSe}_{2}$ monolayers) are promising to realize room temperature ferromagnetic semiconductors. 


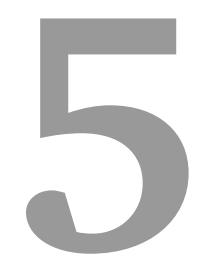

\section{Edge reconstruction of 2D-Xene $(\mathrm{X}=\mathrm{Si}$, $\mathrm{Ge}, \mathrm{Sn})$ nanoribbons}

By performing first principles calculations, we investigate the zigzag edge reconstruction in free-standing $2 D$-Xene $(X=S i, G e, S n)$ nanoribbons. Three different kinds of edge reconstructions $(2 a, 3 a, 4 a)$ are found, in which the reconstruction with $3 a$ periodicity has the lowest energy that opens a gap and shows non-magnetic state. The edge reconstruction can be understood by the band filling of dangling bond states which is affected by the buckling of the structures. From tight binding model, the reduced buckling at the edge after reconstruction decouples $s p^{2}$ and $p_{z}$ orbitals accompanied with a charge transfer from $s p^{2}$ orbitals to $p_{z}$ orbitals.

\subsection{Introduction}

In the wake of graphene, the single-element, monoatomic honeycomb lattices of $2 \mathrm{D}$ $\mathrm{X}$-enes, where $\mathrm{X}=\mathrm{Si}, \mathrm{Ge}$, or Sn (silicene, germanene, stannene), have attracted great attention. Whereas these $2 \mathrm{D}$ layers may be interesting for applications in electronics, scientific interest has also focused on the electronic properties of the $1 \mathrm{D}$ edges of such layers. The reduced symmetry at edges can lead to special electronic edge states. On silicon surfaces, magnetically ordered edge states have been observed, for instance [190].

Calculations on silicene and germanene nanoribbons also predict more exotic electronic states, such as topological edge states, which can be tuned by the ribbon width [191], edge functionalization [192], the substrate [193], and external elec- 
tric [194] or magnetic fields [195]. Edge reconstructions can dramatically alter the electronic properties of the edges. In graphene, edge structures containing StoneWales defects and Klein edges have been observed [196; 197]. Little has been done to systematically map out the edge structures of other Xenens.

The most prominently different edge orientations of honeycomb lattices are called armchair and zigzag edges respectively, see Fig. 5.1. The armchair edge is typically insulating, and thus mostly harmless. The zigzag edge is more interesting, as in its pristine form it is metallic, and as such, it is prone to electronic and structural instabilities [198]. This is best understood for graphene, where the carbon atoms are in-plane $\sigma$-bonded by $s p^{2}$ orbitals, and $\pi$-bonded by out-of-plane $p_{z}$ orbitals. For each carbon atom at the zigzag edge, one $\sigma$-bond is missing, leading to an in-plane $s p^{2}$ dangling bond state. In addition, each such carbon atom carries a non-bonding $p_{z}$ state.

These two states each give a relatively flat edge band containing an unpaired electron, which yields a high density of states (DOS) at the Fermi level. Such a 1D metallic structure is susceptible to structural instabilities. For instance, an edge reconstruction that includes Stone-Wales defects, lifts the dangling bond state away from the Fermi level [199]. Alternatively, if the zigzag edge is passivated by hydrogen atoms, the dangling bond state can be removed completely. This still leaves the $p_{z}$ edge band giving a non-trivial (magnetic) electronic structure, however [200; 201].

The atoms in the honeycomb lattice of silicene, germanene and stanene do not all lie in the same plane. Instead, one has a buckling between the A and B sublattices[202], decreasing the symmetry from $D_{6 h}$ to $D_{3 d}$. This is consistent with the bonding between the atoms being closer to $s p^{3} \sigma$-bonds, which promotes a nonplanar structure. The two states at a zigzag edge discussed above, are still present, but they can now hybridize because of the broken symmetry. The reconstructions of X-ene edges are therefore potentially more diverse than those of graphene.

Computationally, X-ene zigzag edges have been studied with hydrogen passivation of the edge atoms, leaving edge states that might be topological because of the significant spin-orbit coupling in these materials. Such edges are treated as being unreconstructed [203]. Pristine, reconstructed silicene and germanene zigzag edges with $2 a$ periodicity have also been considered [203; 204; 191], where it is believed that these are metallic and show anti-ferromagnetic ground states. Recently, Zhang et al. [205] have studied a germanene layer on top of a $\mathrm{Ge}_{2} \mathrm{Pt}$ substrate by scanning tunneling microscopy and spectroscopy (STM and STS) and have identified reconstructed zigzag edges with a $4 a$ periodicity. The differential conductivity at the edges has a parabolic shape, and indicates that there are no pronounced metallic edge states.

To clarify these inconsistencies, we systematically investigate the zigzag edge reconstruction of pristine silicene, germanene and stanene nano ribbons. Surpisingly, we find that all these edges reconstruct with a $3 a$ periodicity. The $3 a$ peri- 
odicity can in fact be predicted from the band filling of the edge states associated with the buckled pristine edge. The reconstruction opens a gap and leads to nonmagnetic ground states. The change in electronic structure can be analyzed in detail with the help of a tight-binding model.

\subsection{Computational Method}

We performed first principles calculations with density functional theory (DFT) using the all-electron projector augmented wave (PAW) method [109] and a planewave basis set with a cut-off energy of $400 \mathrm{eV}$ as implemented in VASP package [110; 111; 112]. The equilibrium structural and electronic properties for silicene, germanene, and stanene were calculated in the generalized gradient approximation (GGA) [115] as listed in Table 5.1, which agree well with previous work [206]. Zigzag nanoribbons were periodically repeated in the $x$ direction with 9 ribbon chains in $y$ direction to avoid edge interaction and separated by more than $20 \AA$ of vacuum in $y$ and $z$ direction to avoid spurious interaction. We fix the width of nano ribbons to 9 and construct different size of unit cells labelled as $9 \times n(n=1,2,3,4)$ as shown in Fig. 5.1. For the nano ribbons, 3 ribbon chains in the middle are fixed to mimic the corresponding bulk material and the rest atomic structures are fully relaxed until the force less than $0.001 \mathrm{eV} / \AA .45 \times 1 \times 1 \mathrm{k}$-point sampling is used.

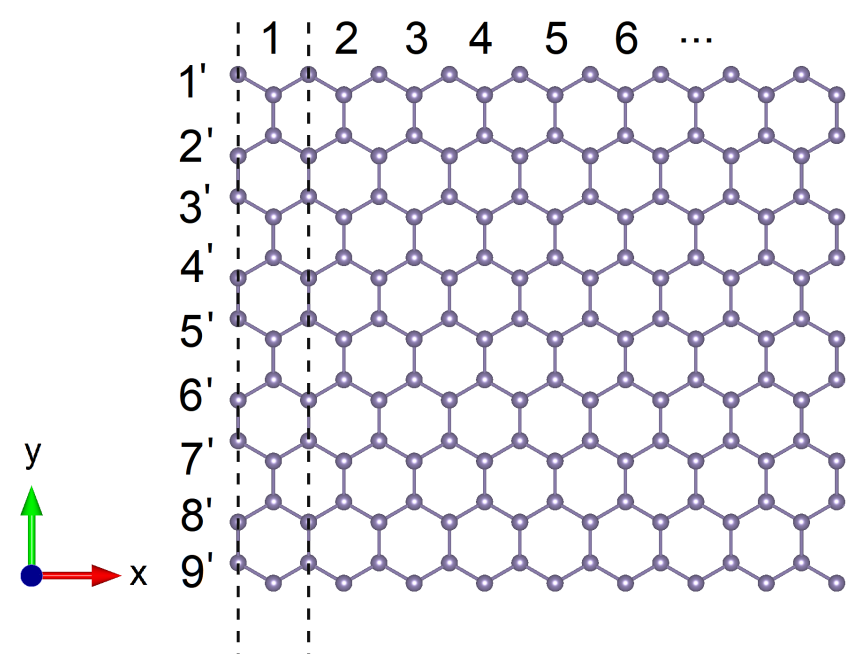

Figure 5.1: (color online). Geometric structures of Germanene nanoribbon nanoribbons. Zigzag edges and armchair edges are along $x$ and $y$ direction, respectively. The dashed rectangle represents $9 \times 1$ unit cell with zigzag edges repeated in $\mathrm{x}$ direction. 
Table 5.1: Calculated structural parameters for 2D-Xene. $a, d_{X-X}$, and $\delta$ represent the lattice constant, bond length, and buckling, respectively.

\begin{tabular}{lccc}
\hline Xene & $a(\AA)$ & $d_{X-X}(\AA)$ & $\delta(\AA)$ \\
\hline Silicene & 3.86 & 2.27 & 0.45 \\
Germanene & 4.05 & 2.44 & 0.69 \\
Stanene & 4.67 & 2.89 & 0.85 \\
\hline
\end{tabular}

\subsection{Electronic properties}
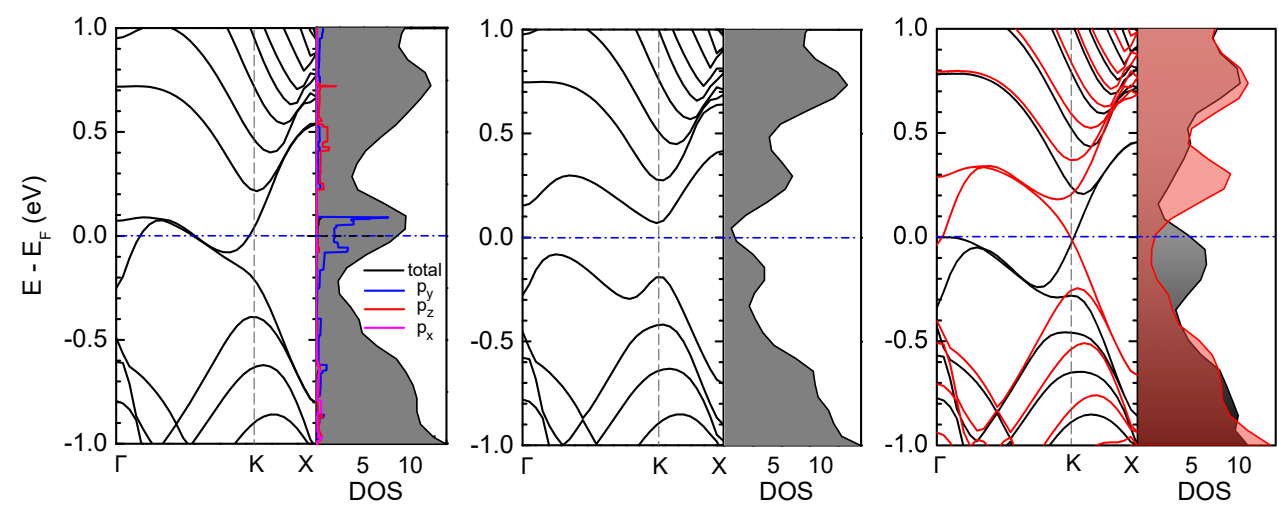

Figure 5.2: (color online). Band structures of $9 \times 1$ zigzag Germanene ribbon with non-spin polarization (a), anti-ferromagnetic polarization (b), ferromagnetic polarization (c). The projected DOS on edge atoms is shown in (a). $K$ point denotes $\frac{1}{3} \pi$.

When 2D nano sheets are cut into 1D ribbons, one missing bond at the zigzag edge atoms gives rise to edge states ( $p_{z}$ orbital) and also the dangling bond states $\left(s p^{2}\right.$ orbital) in the gap. The buckling of the atomic lattice would lead to hybridization between the edge states and the dangling states. The band structures and density of states (DOS) of $9 \times 1$ zigzag Germanene ribbon are plotted in Fig. 5.2. In Fig. 5.2(a), two bands introduced by the two edges are formed in the gap, leaving a DOS peak at the Fermi level. The dispersion of the dangling states $(\Gamma-K)$ indicates the hybridization with the edge states because of the buckling. The orbital resolved DOS on edge atoms in Fig. 5.2(a) shows that the dangling states near the Fermi level mainly consist of $p_{y}$ orbitals, while the edge states formed by $p_{z}$ orbitals are pushed up at $0.5 \mathrm{eV}$. The electronic instability induced by the DOS peak at the Fermi level leads to spin polarized edges. The two edges are coupled antiferromagnetically (AFM) (Fig. 5.2(b)) but only $5 \mathrm{meV} /$ edge atom lower than the ferromagnetic (FM) state (Fig. 5.2(c)) and $25 \mathrm{meV} / \mathrm{edge}$ atom lower than the 
non-magnetic (NM) state. The small difference in energy for AFM and FM states indicates the large ribbon width is sufficient to decouple the two edges.

(a)

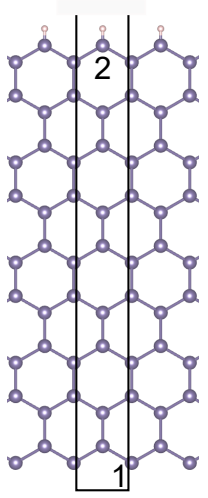

(b)

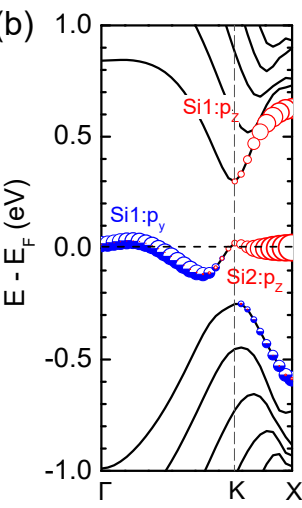

(c)

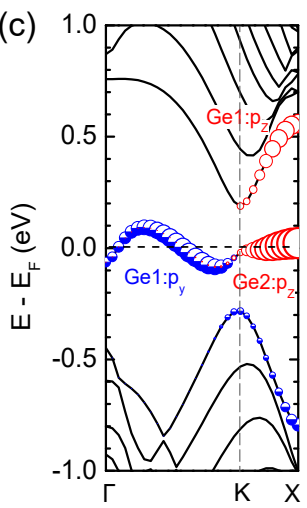

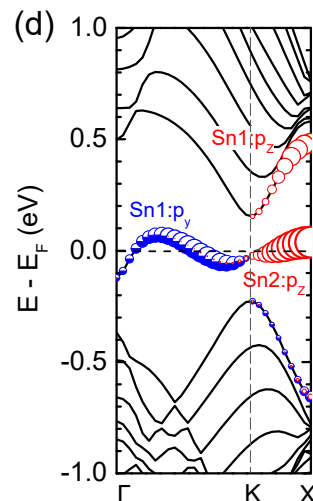

Figure 5.3: (color online). Projected band structures of $9 \times 1$ zigzag silicene (b), germanene (c), and stanene (c) ribbons with hydrogen passivation on edge atom "2".

To have a better understanding of the states introduced by the edges, we compensate the dangling bond on one side of the nano ribbon (edge atom "2" in Fig. 5.3(a)) by hydrogen atoms. From the projected band structure Fig. 5.3(b-d), the dangling bond states of edge atom "1" with $p_{y}$ orbital character lie from $\Gamma$ to $K$ and pull up the edge states $(K-X)$ with $p_{z}$ orbital character by $0.5 \mathrm{eV}$, while the dangling states of the edge atom "2" are passivated by hydrogen and only the flat edge states with $p_{z}$ orbital character lying from $K$ to $X$ are left. If we can further remove the edges states of edge atom "2", we can see that the filling of the edge band that are composed of dangling states and edge states is $1 / 3$, indicating a $3 a$ periodicity.

\subsection{Edge reconstruction}

Table 5.2: Edge energy (eV) for relaxed silicence, germanene, and stanene zigzag ribbons with different periodicity.

\begin{tabular}{lccc}
\hline $\mathrm{n}$ & 2 & 3 & 4 \\
\hline$E_{\text {edge }}($ Silicene $)$ & -0.37 & -0.43 & -0.33 \\
$E_{\text {edge }}($ Germanene $)$ & -0.43 & -0.51 & -0.43 \\
$E_{\text {edge }}($ Stanene) & -0.22 & -0.35 & -0.23 \\
\hline
\end{tabular}

The electronic instability at the zigzag of 2D-Xene not only lead to breaking of the time reversal symmetry but also the spatial symmetry, namely, the edge re- 
(a) $\quad \mathrm{Si}(2 \mathrm{a})$
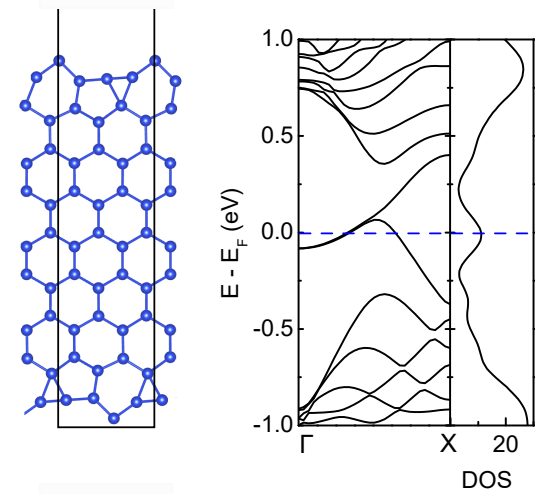

(c) $\mathrm{Ge}(2 \mathrm{a})$
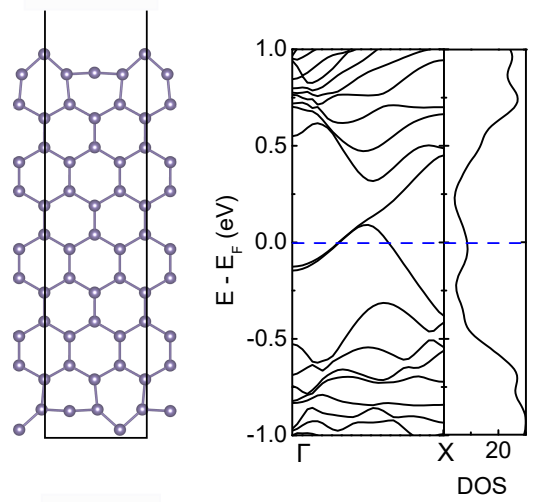

(e) $\operatorname{Sn}(2 \mathrm{a})$

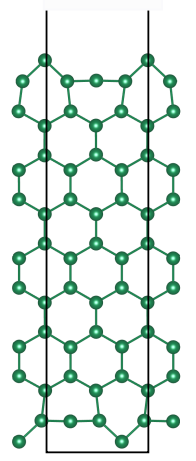

(b)

Si (3a)
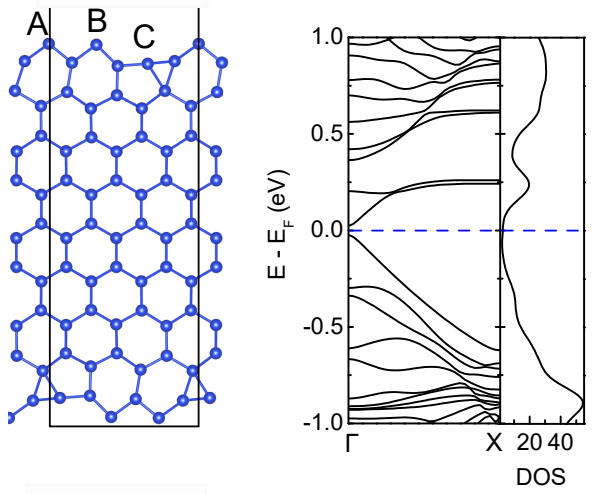

(d)

$\mathrm{Ge}(3 \mathrm{a})$
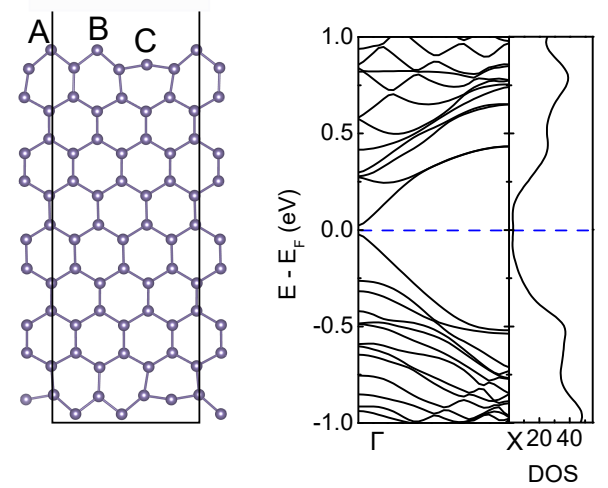

(f)

$\operatorname{Sn}(3 a)$
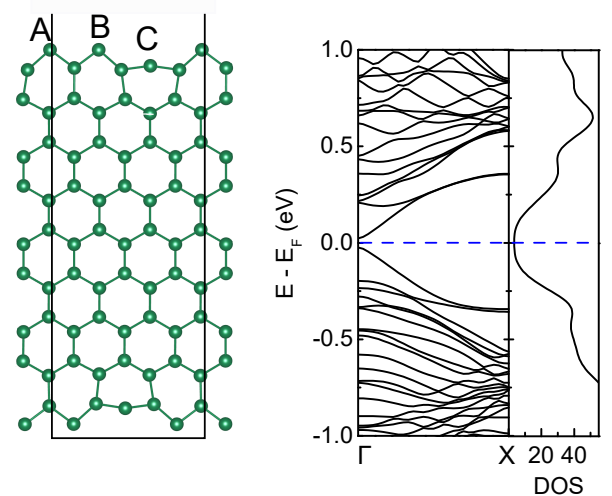

Figure 5.4: (color online). Band structures and atomic structures of relaxed zigzag ribbon with $2 a$ and $3 a$ periodicity for silicene (a,b), germanene (c,d), and stanene $(\mathrm{e}, \mathrm{f})$, respectively. A, B, C label the edge atoms in structures with $3 a$ periodicity. 
construction. We relaxed the structures of germanene nanoribbons with different periodicity $9 \times n(n=2,3,4)$. The structure reconstruction greatly decreases the total energy with respect to the small energy difference of different states shown in Fig. 4.1. Therefore, we first relax the structure with NM state, and then start from the pre-relaxed structure, we do further relaxation with FM or AFM state to find the ground-state structure. We define the edge energy as

$$
E_{\text {edge }}=\left(E_{\text {relaxed }}-E_{\text {unrelaxed }}\right) / n
$$

in which $E_{\text {relaxed }}$ and $E_{\text {unrelaxed }}$ are the total energy for the relaxed and unrelaxed germanene ribbon, respectively, and $\mathrm{n}$ is the size of the unit cell defined above. The relaxation generates three different kinds of periodicity: $2 a, 3 a$ and $4 a$. Comparing the edge energies with different periodicity in Table 5.2, structures with $3 a$ periodicity have the lowest edge energies, which is consistent with the band filling in Fig. 5.3. For comparison, the relaxed atomic and electronic structures with $2 a$ and $3 a$ periodicity are plotted in Fig. 5.4. With $2 a$ periodicity, two degenerate states near the Fermi level give rise to a DOS peak at Fermi level, leading to an electronic instability. Two edges show anti-ferromagnetic coupling as reported in previous work [203; 204; 191]. For relaxed structures with $3 a$ periodicity, a gap opens near Fermi level which further lowers the energy with respect to the structures with $2 a$ periodicity. The ground states are insulating and non-magnetic.

In $1 \mathrm{D}$ atom chain, $1 / 3$ filled band would lead to a Peierls distortion in which three atoms come close and form trimer to open a gap. However, we have a $1 \mathrm{D}$ nano ribbon with finite ribbon width. As two edge atoms (A,B) come close, the bonds between edge atoms and inner atoms would push the third edge atom (C) down. This is also happened on many crystal surface, such as Si (100) surface [207]. With reconstruction, edge atom A and B move towards each other with mainly $p_{y}$ orbital. Edge atom $\mathrm{C}$ has an increased occupation of $p_{x}$ orbital. It is easy to understand that edge atom C move inwards and prefer to occupy $p_{x}$ orbital which can form $\sigma$ bonds with its neighbours. Actually, there has not too much meaning to discuss the individual orbitals on each atoms. Because the $s p^{2}$ or $s p^{3}$ hybridization makes the behaviour of electrons more complicated.

\subsection{Tight binding model}

To understand the 3a periodicity of edge reconstruction, we constructed a tight binding model based on the maximally localized wannier function. The bloch wavefunction are projected onto atom-centered $p_{z}$ orbitals on each site for the $\pi$ and $\pi^{*}$ bands and atom-centered $s p^{2}$ orbitals for the $\sigma$ bonding. We just focus on germanene monolayer, the $8 \times 8$ tight binding Hamiltonian is constructed with one $p_{z}$ 

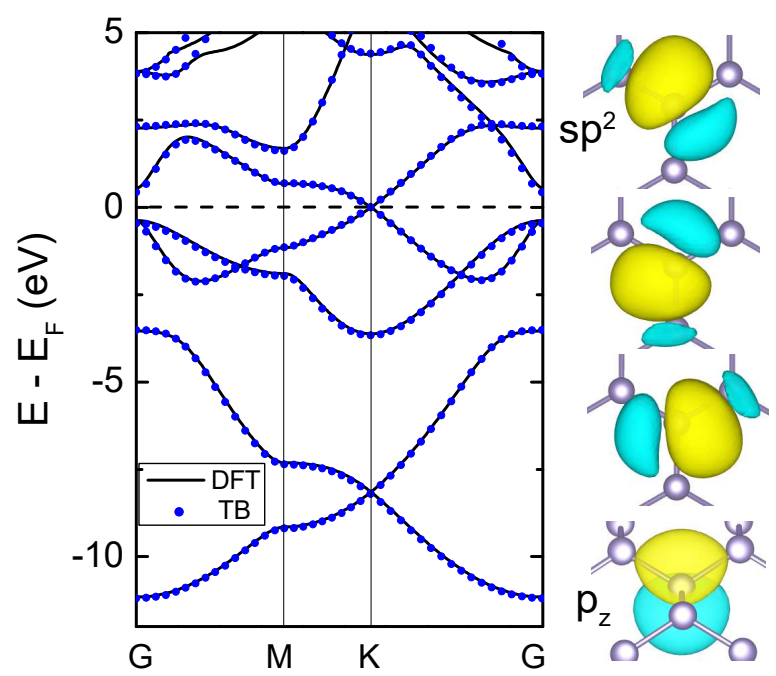

Figure 5.5: (color online). Comparision of bands structures of germanene monolayer from DFT calculation and TB model based on Wannier function basis. Surface plot for the maximally localized $s p^{2}$ and $p_{z}$ orbital Wannier functions. The yellow and blue regions indicate positive and negative values of the real part of the wavefunction amplitudes.

orbitals and three $s p^{2}$ on each Ge atom in primitive cell:

$$
H(\boldsymbol{k})=\left(\begin{array}{ll}
H_{A A}(\boldsymbol{k}) & H_{A B}(\boldsymbol{k}) \\
H_{B A}(\boldsymbol{k}) & H_{B B}(\boldsymbol{k})
\end{array}\right)
$$

The Bloch wave functions resulting from VASP calculation are transformed to an orthogonal set of wannier functions by

$$
\left|\psi_{\boldsymbol{k} \alpha}\right\rangle=\frac{1}{\sqrt{N}} \sum_{\boldsymbol{R}} e^{i \boldsymbol{k} \cdot\left(\boldsymbol{R}+\tau_{\alpha}\right)}\left|\boldsymbol{R}+\tau_{\alpha}\right\rangle
$$

in which index $\alpha$ represents the A or B sublattice and $\left|\boldsymbol{R}+\tau_{\alpha}\right\rangle$ denotes the Wannier function for different orbitals at site $\boldsymbol{R}+\tau_{\alpha}$. In the tight binding Hamiltonian, the hopping parameters can be expressed in the form of Wannier function by

$$
\begin{aligned}
H_{\alpha \beta}(\boldsymbol{k}) & =\left\langle\psi_{\boldsymbol{k} \alpha}|H| \psi_{\boldsymbol{k} \beta}\right\rangle \\
& =\frac{1}{N} \sum_{\boldsymbol{R} \boldsymbol{R}^{\prime}} e^{i \boldsymbol{k} \cdot\left(\boldsymbol{R}+\tau_{\alpha}-\boldsymbol{R}^{\prime}-\tau_{\beta}\right)}\left\langle\boldsymbol{R}+\tau_{\alpha}|H| \boldsymbol{R}^{\prime}+\tau_{\beta}\right\rangle
\end{aligned}
$$

in which $\left\langle\boldsymbol{R}+\tau_{\alpha}|H| \boldsymbol{R}^{\prime}+\tau_{\beta}\right\rangle=t_{\alpha \beta}\left(\boldsymbol{R}-\boldsymbol{R}^{\prime}\right)$. The bandstructure for germanene monolayer based on wannier function basis fit quite well with that from DFT calculations in the energy range we are interested at as shown in Fig. 5.5. 
The hopping parameters for the nearest neighbours in the unit cell are listed in Table 5.3. We can see that the buckling in gemanene introduces the hopping between $s p^{2}$ and $p_{z}$ orbitals $\left(\mathrm{t}_{\sigma \pi}\right)$, leading to a mix of $s p^{2}$ and $p_{z}$ orbitals. On the unrelaxed edge, the appearance of dangling bond increases the hopping between $s p^{2}$ orbitals $\left(\mathrm{t}_{\sigma \sigma}\right)$. After the edge reconstruction, two edge atoms (Ge A and B) move together and one ( $\mathrm{Ge} \mathrm{C}$ ) moves down to the inner part. The reduced buckling of the edge atoms result in a decrease of $\mathrm{t}_{\sigma \pi}$ accompanied with an increase of $\mathrm{t}_{\pi \pi}$ and a decrease of $\mathrm{t}_{\sigma \sigma}$. This indicates a reduced hybridization between $s p^{2}$ and $p_{z}$ orbitals and a charge transfer from $s p^{2}$ to $p_{z}$ orbitals. The relative large hopping between different $s p^{2}$ orbitals on edge atoms (numbers in the parenthesis) indicate enhanced $\sigma$ bonds between edge atoms and their nearest neighbours. This can also be seen from the reduced bond length between $\mathrm{Ge} C$ and its nearest neighbours $(2.40 \AA)$. As a result, this Peierls-like edge reconstruction opens a gap near the Fermi level as seen in Fig. 5.4. The buckling of the Ge atoms determines the hopping between $s p^{2}$ and $p_{z}$ orbitals and affects the bonding interaction between $\mathrm{Ge}$ atoms.

Table 5.3: Hopping integrals $t(\mathrm{eV})$ and buckling $\delta(\AA)$ of monolayer germanene, unrelaxed zigzag edge, relaxed $8 \times 3$ zigzag edge with three edge atoms (A, B, C). The numbers in the parenthesis indicate the hopping between different $s p^{2}$ orbitals on edge atoms. Only the nearest hopping parameters are shown in the table.

\begin{tabular}{lcccccc}
\hline & $\epsilon_{s p^{2}}$ & $\epsilon_{p_{z}}$ & $\mathrm{t}_{\sigma \sigma}$ & $\mathrm{t}_{\pi \pi}$ & $\mathrm{t}_{\sigma \pi}$ & $\delta$ \\
\hline germanene & -1.06 & 1.00 & -3.20 & -0.68 & \pm 1.11 & 0.69 \\
unrelaxed edge & -1.19 & 0.78 & -3.74 & -0.66 & \pm 1.11 & 0.69 \\
Ge A & -1.21 & 0.83 & $-3.61(-1.88)$ & -0.94 & \pm 0.47 & 0.46 \\
Ge B & -1.22 & 0.84 & $-3.61(-1.82)$ & -0.97 & \pm 0.39 & 0.42 \\
Ge C & -1.73 & 0.49 & $-3.43(-2.62)$ & -0.78 & \pm 0.94 & 0.58 \\
\hline
\end{tabular}

\subsection{Discussion}

The periodicity of the edge reconstruction is a result of the perels-like distortion associated with the band filling of dangling bond states near the Fermi level. And the band filling of dangling bond states is determined by the interaction between dangling states and edge states, resulting from the buckling of the structure. In flat graphene edges, the $50 \%$ band filling of dangling states (one unpaired electron in the dangling bond state) leads to a Stone-Wales reconstruction, in which two edge atom come together and form a bond to passivate the dangling bond. In 2D-Xene $(\mathrm{X}=\mathrm{Si}, \mathrm{Ge}, \mathrm{Sn}$ ), due to the buckling, dangling states pull up the edge states and has a small band filling. Especially, in germanene ribbons, 1/3 band occupation leads to a edge reconstruction with $3 a$ periodicity. We noticed that the sillicene 
ribbons with a small buckling ( $0.45 \AA$ ) have a band filling of 0.40 indicated from Fig. 5.3(b). To passivate the extra electrons on the edges, the edge atom $\mathrm{C}$ forms an extra bond with inner atoms (see Fig. 5.4(b)) and gives rise to a $3 a$ periodicity at the zigzag edge. In Fig. 5.3(d), the band filling of stanene edge states is slightly less than $1 / 3$ for its large buckling $(0.85 \AA)$. A charge transfer from the inner $\mathrm{Sn}$ atoms to the edges were found, which makes the zigzag edges fulfill the one-third band filling. By applying strain, we can tune the buckling of the structures. In germanene zigzag edges, 3.5\% compressive strain changes the band filling from $1 / 3$ to $1 / 4$ and leads to a $4 a$ periodicity with a significant enhanced hybridization of $s p^{2}$ and $p_{z}$ orbitals, while $8.5 \%$ tensile strain gives rise to to a $2 a$ periodicity.

The substrate material can also influence the buckling within the 2D layer, resulting from the Van der Waals interaction between them. Zhang et al. reported the germanene grown on the $\mathrm{Ge}_{2} \mathrm{Pt}$ substrate with a lattice constant of $4.3 \AA$ and a relative small buckling of $0.2 \AA[205]$. However, they found a $4 a$ periodicity at the zigzag edge of germanene. It should be noted that our calculations are performed at zero Kelvin, while most of the experiment are done at room temperature. We see that the edge energy difference between the relaxed structures with different periodicity is on the scale of a few tens meV. At room temperature, the thermal energy $(\sim 25 \mathrm{meV})$ can affect the periodicity of the zigzag edges. In addition, possible charge transfer between the substrate and the material would change the edge band filling and so the edge reconstruction.

\subsection{Conclusion}

In this Chapter, by performing first principles calculations, we investigate the zigzag edge reconstruction in 2D-Xene ( $\mathrm{X}=\mathrm{Si}, \mathrm{Ge}, \mathrm{Sn}$ ) nanoribbons. Three different kinds of edge reconstruction are found. The ground-state structures have $3 a$ periodicity, which opens a gap at the Fermi level and shows non-magnetic ground states. The edge reconstruction is determined by the band filling of the dangling states associated with the buckling of the atomic lattice. 


\section{Bibliography}

[1] K. S. Novoselov, A. K. Geim, S. V. Morozov, D. Jiang, Y. Zhang, S. V. Dubonos, I. V. Grigorieva, and A. A. Firsov, "Electric Field Effect in Atomically Thin Carbon Films", Science 306, 666-669 (2004).

[2] K. S. Novoselov, A. K. Geim, S. V. Morozov, D. Jiang, M. I. Katsnelson, I. V. Grigorieva, S. V. Dubonos, and A. A. Firsov, "Two-dimensional gas of massless Dirac fermions in graphene", Nature (London) 438, 197-200 (2005).

[3] K. Nomura and A. H. MacDonald, "Quantum hall ferromagnetism in graphene", Phys. Rev. Lett. 96, 256602 (2006).

[4] Y. Cao, V. Fatemi, S. Fang, K. Watanabe, T. Taniguchi, E. Kaxiras, and P. JarilloHerrero, "Unconventional superconductivity in magic-angle graphene superlattices", Nature (London) 556, 43-50 (2018).

[5] F. Liu, J. Zhou, C. Zhu, and Z. Liu, "Electric field effect in two-dimensional transition metal dichalcogenides", Adv. Funct. Mater. 27, 1602404 (2017).

[6] L. R. Radovic and B. Bockrath, "On the chemical nature of graphene edges: Origin of stability and potential for magnetism in carbon materials", Journal of the American Chemical Society 127, 5917-5927 (2005).

[7] S. Feng, Z. Lin, X. Gan, R. Lvb, and M. Terrones, "Doping two-dimensional materials: ultra-sensitive sensors, band gap tuning and ferromagnetic monolayers", Nanoscale Horizons 2(2), 72-80 (2017).

[8] P.-C. Yeh, W. Jin, N. Zaki, D. Zhang, J. T. Liou, J. T. Sadowski, A. Al-Mahboob, J. I. Dadap, I. P. Herman, P. Sutter, , and R. M. O. Jr., "Layer-dependent electronic structure of an atomically heavy two-dimensional dichalcogenide”, Phys. Rev. B 91, 041407(R) (2015).

[9] A. Kimouche, M. M. Ervasti, R. Drost, S. Halonen, A. Harju, P. M. Joensuu, J. Sainio, and P. Liljeroth, "Ultra-narrow metallic armchair graphene nanoribbons", Nat. Commun. 6, 10177 (2015).

[10] L. Liu, T. Moriyama, D. C. Ralph, and R. A. Buhrman, "Spin-Torque Ferromagnetic Resonance Induced by the Spin Hall Effect”, Phys. Rev. Lett. 106, 036601 (2011).

[11] K. F. Mak, C. Lee, J. Hone, J. Shan, and T. F. Heinz, "Atomically Thin $\mathrm{MoS}_{2}$ : A New Direct-Gap Semiconductor”, Phys. Rev. Lett. 105, 136805 (2010). 
[12] D. Xiao, G.-B. Liu, W. Feng, X. Xu, and W. Yao, "Coupled spin and valley physics in monolayers of $\mathrm{MoS}_{2}$ and other group-VI dichalcogenides", Phys. Rev. Lett. 108, 196802 (2012).

[13] A. Ohtomo and H. Y. Hwang, "A high-mobility electron gas at the $\mathrm{LaAlO}_{3} / \mathrm{SrTiO}_{3}$ heterointerface", Nature (London) 427, 423-426 (2004).

[14] N. Nakagawa, H. Y. Hwang, and D. A. Muller, "Why some interfaces cannot be sharp", Nat. Mater. 5, 204-209 (2006).

[15] P. Hohenberg and W. Kohn, "Inhomogeneous electron gas", Phys. Rev. 136, B864 B871 (1964).

[16] W. Kohn and L. J. Sham, "Self-consistent equations including exchange and correlation effects", Phys. Rev. 140, A1133-A1138 (1965).

[17] J.-S. Lee, Y. W. Xie, H. K. Sato, C. Bell, Y. Hikita, H. Y. Hwang, and C.-C. Kao, "Titanium $\mathrm{d}_{x y}$ ferromagnetism at the $\mathrm{LaAlO}_{3} / \mathrm{SrTiO}_{3}$ interface", Nat. Mater. 12, 703-706 (2013).

[18] A. Brinkman, M. Huijben, M. van Zalk, J. Huijben, U. Zeitler, J. C. Maan, W. G. van der Wiel, G. Rijnders, D. H. A. Blank, and H. Hilgenkamp, "Magnetic effects at the interface between non-magnetic oxides", Nat. Mater. 6, 493-496 (2007).

[19] S. Gariglio, N. Reyren, A. D. Caviglia, and J.-M. Triscone, "Superconductivity at the $\mathrm{LaAlO}_{3} / \mathrm{SrTiO}_{3}$ interface”, J. Phys.: Condens. Matter 21, 164213 (2009).

[20] J. A. Bert, B. Kalisky, C. Bell, M. Kim, Y. Hikita, H. Y. Hwang, and K. A. Moler, "Direct imaging of the coexistence of ferromagnetism and superconductivity at the $\mathrm{LaAlO}_{3} / \mathrm{SrTiO}_{3}$ interface", Nat. Phys. 7, 767-771 (2011).

[21] S. Thiel, G. Hammerl, A. Schmehl, C. W. Schneider, and J. Mannhart, "Direct imaging of the coexistence of ferromagnetism and superconductivity at the $\mathrm{LaAlO}_{3} / \mathrm{SrTiO}_{3}$ interface", Nat. Phys. 7, 767-771 (2011).

[22] P. Delugas, A. Filippetti, V. Fiorentini, D. I. Bilc, D. Fontaine, and P. Ghosez, "Spontaneous 2-dimensional carrier confinement at the $n$-type $\mathrm{SrTiO}_{3} / \mathrm{LaAlO}_{3}$ interface", Phys. Rev. Lett. 106, 166807 (2011).

[23] C. W. Bark, D. A. Felker, Y. Wang, Y. Zhang, H. W. Jang, C. M. Folkman, J. W. Park, S. H. Baek, H. Zhou, D. D. Fong, X. Q. Pan, E. Y. Tsymbal, M. S. Rzchowski, and C. B. Eom, "Tailoring a two-dimensional electron gas at the $\mathrm{LaAlO}_{3} / \mathrm{SrTiO}_{3}(001)$ interface by epitaxial strain", Proc. Natl. Acad. Sci. U.S.A. 108, 4720-4724 (2011).

[24] Y. Z. Chen, F. Trier, T. Wijnands, R. Green, N. Gauquelin, R. Egoavil, D. V. Christensen, G. Koster, M. Huijben, N. Bovet, et al., "Extreme mobility enhancement of two-dimensional electron gases at oxide interfaces by charge-transfer-induced modulation doping", Nat. Mater. 14, 801-806 (2015). 
[25] E. Lesne, N. Reyren, D. Doennig, R. Mattana, H. Jaffrès, V. Cros, F. Petroff, F. Choueikani, P. Ohresser, R. Pentcheva, et al., "Suppression of the critical thickness threshold for conductivity at the $\mathrm{LaAlO}_{3} / \mathrm{SrTiO}_{3}$ interface", Nat. Communications 5, 4291 (2014).

[26] H. Kim, N. Y. Chan, J.-y. Dai, and D.-W. Kim, "Enhanced surface-and-interface coupling in pd-nanoparticle-coated $\mathrm{LaAlO}_{3} / \mathrm{SrTiO}_{3}$ heterostructures: strong gas-and photo-induced conductance modulation", Scientific Reports 5, 8531 (2015).

[27] M. Rossle, K. W. Kim, A. Dubroka, P. Marsik, C. N. Wang, R. Jany, C. Richter, J. Mannhart, C. W. Schneider, A. Frano, P. Wochner, Y. Lu, B. Keimer, D. K. Shukla, J. Strempfer, and C. Bernhard, "Electric-field-induced polar order and localization of the confined electrons in $\mathrm{LaAlO}_{3} / \mathrm{SrTiO}_{3}$ heterostructures", Phys. Rev. Lett. 110, 136805 (2013).

[28] P. P. Aurino, A. Kalabukhov, N. Tuzla, E. Olsson, A. Klein, P. Erhart, Y. A. Boikov, I. T. Serenkov, V. I. Sakharov, T. Claeson, and D. Winkler, "Reversible metal-insulator transition of ar-irradiated $\mathrm{LaAlO}_{3} / \mathrm{SrTiO}_{3}$ interfaces", Phys. Rev. B 92, 155130 (2015).

[29] K. Au, D. Li, N. Chan, and J. Dai, "Polar liquid molecule induced transport property modulation at $\mathrm{LaAlO}_{3} / \mathrm{SrTiO}_{3}$ heterointerface", Adv. Mater. 24, 2598-2602 (2012).

[30] Z. Huang, Z. Q. Liu, M. Yang, S. W. Zeng, A. Annadi, W. M. Lu, X. L. Tan, P. F. Chen, L. Sun, X. Renshaw Wang, Y. L. Zhao, C. J. Li, J. Zhou, K. Han, W. B. Wu, Y. P. Feng, J. M. D. Coey, T. Venkatesan, and Ariando, "Biaxial strain-induced transport property changes in atomically tailored $\mathrm{SrTiO}_{3}$-based systems", Phys. Rev. B 90, 125156 (2014).

[31] P. Kumar, P. Pal, A. K. Shukla, J. J. Pulikkotil, and A. Dogra, "Metal-to-insulator transition in $\mathrm{LaAl}_{1-x} \mathrm{Cr}_{x} \mathrm{O}_{3} / \mathrm{SrTiO}_{3}$ oxide heterostructures guided by electronic reconstruction", Phys. Rev. B 91, 115127 (2015).

[32] C. Ahn, J.-M. Triscone, and J. Mannhart, "Electric field effect in correlated oxide systems", Nature (London) 424, 1015 (2003).

[33] Y. C. Liao, T. Kopp, C. Richter, A. Rosch, and J. Mannhart, "Metal-insulator transition of the $\mathrm{LaAlO}_{3} / \mathrm{SrTiO}_{3}$ interface electron system”, Phys. Rev. B 83, 075402 (2011).

[34] H. Peelaers, K. Krishnaswamy, L. Gordon, D. Steiauf, A. Sarwe, A. Janotti, and C. G. Van de Walle, "Impact of electric-field dependent dielectric constants on two-dimensional electron gases in complex oxides", Appl. Phys. Lett. 107, 183505 (2015).

[35] S. K. Kim, S.-I. Kim, H. Lim, D. S. Jeong, B. Kwon, S.-H. Baek, and J.-S. Kim, "Electricfield-induced shift in the threshold voltage in $\mathrm{LaAlO}_{3} / \mathrm{SrTiO}_{3}$ heterostructures", Scientific Reports 5, 8023 (2015).

[36] L. Kim, J. Kim, D. Jung, J. Lee, and U. V. Waghmare, "Polarization of strained $\mathrm{BaTiO}_{3} / \mathrm{SrTiO}_{3}$ artificial superlattice: First-principles study", Appl. Phys. Lett. 87, 052903 (2005). 
[37] P. Zubko, N. Stucki, C. Lichtensteiger, and J.-M. Triscone, "X-ray diffraction studies of $180^{\circ}$ ferroelectric domains in $\mathrm{PbTiO}_{3} / \mathrm{SrTiO}_{3}$ superlattices under an applied electric field", Phys. Rev. Lett. 104, 187601 (2010).

[38] L. Kim, J. Kim, U. V. Waghmare, D. Jung, and J. Lee, "Structural transition and dielectric response of an epitaxially strained $\mathrm{BaTiO}_{3} / \mathrm{SrTiO}_{3}$ superlattice: A firstprinciples study", Phys. Rev. B 72, 214121 (2005).

[39] S.-I. Kim, D.-H. Kim, Y. Kim, S. Y. Moon, M.-G. Kang, J. K. Choi, H. W. Jang, S. K. Kim, J.-W. Choi, S.-J. Yoon, et al., "Non-volatile control of 2DEG conductivity at oxide interfaces", Adv. Mater. 25, $4612-4617$ (2013).

[40] K. D. Fredrickson and A. A. Demkov, "Switchable conductivity at the ferroelectric interface: Nonpolar oxides", Phys. Rev. B 91, 115126 (2015).

[41] M. K. Niranjan, J. D. Burton, J. P. Velev, S. Jaswal, and E. Y. Tsymbal, "Magnetoelectric effect at the $\mathrm{SrRuO}_{3} / \mathrm{BaTiO}_{3}$ (001) interface: An ab initio study", Appl. Phys. Lett. 95, 052501 (2009).

[42] J. Junquera and P. Ghosez, "Critical thickness for ferroelectricity in perovskite ultrathin films", Nature (London) 422, 506- (2003).

[43] E. A. Giess, R. L. Sandstrom, W. J. Gallagher, A. Gupta, S. L. Shinde, R. F. Cook, E. I. Cooper, E. J. M. O’Sullivan, J. M. Roldan, A. P. Segmuller, and J. Angilello, "Lanthanide gallate perovskite-type substrates for epitaxial, high-tc superconducting $\mathrm{Ba}_{2} \mathrm{YCu}_{3} \mathrm{O}_{7-\delta}$ films", IBM Journal of Research and Development 34, 916-926 (1990).

[44] C. Li, D. Cui, Y. Zhou, H. Lu, Z. Chen, D. Zhang, and F. Wu, "Asymmetric rocking curve study of the crystal structure orientations for $\mathrm{BaTiO}_{3}$ thin films grown by pulsed laser deposition", Appl. Surf. Sci. 136, 173 - 177 (1998).

[45] M. Cardona, "Optical properties and band structure of $\mathrm{SrTiO}_{3}$ and $\mathrm{BaTiO}_{3}$ ", Phys. Rev. 140, A651-A655 (1965).

[46] A. Dubroka, M. Rössle, K. W. Kim, V. K. Malik, L. Schultz, S. Thiel, C. W. Schneider, J. Mannhart, G. Herranz, O. Copie, M. Bibes, A. Barthélémy, and C. Bernhard, "Dynamical response and confinement of the electrons at the $\mathrm{LaAlO}_{3} / \mathrm{SrTiO}_{3}$ interface", Phys. Rev. Lett. 104, 156807 (2010).

[47] S. Nazir, M. Behtash, and K. Yang, "Enhancing interfacial conductivity and spatial charge confinement of $\mathrm{LaAlO}_{3} / \mathrm{SrTiO}_{3}$ heterostructures via strain engineering", Appl. Phys. Lett. 105, 141602 (2014).

[48] R. Pentcheva and W. E. Pickett, "Avoiding the polarization catastrophe in $\mathrm{LaAlO}_{3}$ overlayers on $\mathrm{SrTiO}_{3}$ (001) through polar distortion”, Phys. Rev. Lett. 102, 107602 (2009).

[49] W. Zhong, R. D. King-Smith, and D. Vanderbilt, "Giant lo-to splittings in perovskite ferroelectrics", Phys. Rev. Lett. 72, 3618-3621 (1994). 
[50] J. P. Velev, C.-G. Duan, J. D. Burton, A. Smogunov, M. K. Niranjan, E. Tosatti, S. S. Jaswal, and E. Y. Tsymbal, "Magnetic tunnel junctions with ferroelectric barriers: Prediction of four resistance states from first principles", 9, 427-432 (2009).

[51] G. Gerra, A. K. Tagantsev, N. Setter, and K. Parlinski, "Ionic polarizability of conductive metal oxides and critical thickness for ferroelectricity in $\mathrm{BaTiO}_{3}$ ", Phys. Rev. Lett. 96, 107603 (2006).

[52] J. Lee and A. A. Demkov, "Charge origin and localization at the n-type $\mathrm{SrTiO}_{3} / \mathrm{LaAlO}_{3}$ interface”, Phys. Rev. B 78, 193104 (2008).

[53] J. Junquera, M. H. Cohen, and K. M. Rabe, "Nanoscale smoothing and the analysis of interfacial charge and dipolar densities", J. Phys.: Condens. Matter 19, 213203 (2007).

[54] J. Lee and A. A. Demkov, "Theoretical investigation of the band alignment at the $\mathrm{LaAlO}_{3} / \mathrm{SrTiO}_{3}$ interface”, MRS Proceedings 966 (2006).

[55] M. Itoh, R. Wang, Y. Inaguma, T. Yamaguchi, Y.-J. Shan, and T. Nakamura, "Ferroelectricity induced by oxygen isotope exchange in strontium titanate perovskite", Phys. Rev. Lett. 82, 3540-3543 (1999).

[56] S. Chae, W. Choi, H. Yoo, and B. Kang, "Metal-insulator-like transition in the $\mathrm{LaAlO}_{3} / \mathrm{BaTiO}_{3}$ interface", Current Applied Physics 11, 521-524 (2011).

[57] H. Ohno, H. Munekata, T. Penney, S. von Molnár, and L. L. Chang, "Magnetotransport properties of $p$-type (In,Mn)As diluted magnetic III-V semiconductors", Phys. Rev. Lett. 68, 2664-2667 (1992).

[58] H. Ohno, A. Shen, F. Matsukura, A. Oiwa, A. Endo, S. Katsumoto, and Y. Iye, "(Ga,Mn)As: A new diluted magnetic semiconductor based on GaAs", Appl. Phys. Lett. 69, 363-365 (1996).

[59] T. Dietl, H. Ohno, F. Matsukura, J. Cibert, and D. Ferrand, "Zener model description of ferromagnetism in zinc-blende magnetic semiconductors", Science 287, 10191022 (2000).

[60] T. Dietl, "A ten-year perspective on dilute magnetic semiconductors and oxides", Nat. Mater. 9, 965-974 (2010).

[61] T. Jungwirth, J. Sinova, J. Mašek, J. Kučera, and A. H. MacDonald, "Theory of ferromagnetic (III,Mn)V semiconductors", Rev. Mod. Phys. 78, 809-864 (2006).

[62] K. Sato, L. Bergqvist, J. Kudrnovský, P. H. Dederichs, O. Eriksson, I. Turek, B. Sanyal, G. Bouzerar, H. Katayama-Yoshida, V. A. Dinh, T. Fukushima, H. Kizaki, and R. Zeller, "First-principles theory of dilute magnetic semiconductors", Rev. Mod. Phys. 82, 1633-1690 (2010).

[63] N. D. Mermin and H. Wagner, "Absence of Ferromagnetism or Antiferromagnetism in One- or Two-Dimensional Isotropic Heisenberg Models", Phys. Rev. Lett. 17, 11331136 (1966). 
[64] P. C. Hohenberg, "Existence of long-range order in one and two dimensions", Phys. Rev. 158, 383-386 (1967).

[65] in Ultrathin Magnetic Structures I-IV, edited by J. A. C. Bland and B. Heinrich (Springer-Verlag, Berlin) (1994-2005).

[66] C. Gong, L. Li, Z. Li, H. Ji, A. Stern, Y. Xia, T. Cao, W. Bao, C. Wang, Y. Wang, Z. Q. Qiu, R. J. Cava, S. G. Louie, J. Xia, and X. Zhang, "Discovery of intrinsic ferromagnetism in two-dimensional van der Waals crystals", Nature (London) 546, 265-269 (2017).

[67] B. Huang, G. Clark, E. Navarro-Moratalla, D. R. Klein, R. Cheng, K. L. Seyler, D. Zhong, E. Schmidgall, M. A. McGuire, D. H. Cobden, W. Yao, D. Xiao, P. JarilloHerrero, and $\mathrm{X}$. Xu, "Layer-dependent ferromagnetism in a van der Waals crystal down to the monolayer limit", Nature (London) 546, 270-273 (2017).

[68] N. Samarth, "Magnetism in flatland", Nature (London) 546, 216-218 (2017).

[69] K. S. Novoselov, D. Jiang, F. Schedin, T. J. Booth, V. V. Khotkevich, S. V. Morozov, and A. K. Geim, "Two-dimensional atomic crystals", Proc. Natl. Acad. Sci. U.S.A. 102, 10451-10453 (2005).

[70] K. S. Novoselov, V. I. Fal'ko, L. Colombo, P. R. Gellert, M. G. Schwab, and K. Kim, "A roadmap for graphene", Nature (London) 490, 192-200 (2012).

[71] A. Splendiani, L. Sun, Y. Zhang, T. Li, J. Kim, C. Chim, G. Galli, and F. Wang, "Emerging photoluminescence in monolayer $\mathrm{MoS}_{2}$ ", Nano Letters 10, 1271-1275 (2010).

[72] B. Radisavljevic, A. Radenovic, J. Brivio, V. Giacometti, and A. Kis, "Single-layer $\mathrm{MoS}_{2}$ transistors", Nat. Nanotechnol. 6, 147-150 (2011).

[73] A. K. Geim and I. V. Grigorieva, "Van der Waals heterostructures", Nature (London) 499, 419-425 (2013).

[74] M. Gibertini, M. Koperski, A. F. Morpurgo, and K. S. Novoselov, "Magnetic 2D materials and heterostructures", Nat. Nanotechnol. 14, 408-419 (2019).

[75] M. Bonilla, S. Kolekar, Y. Ma, H. C. Diaz, V. Kalappattil, R. Das, T. Eggers, H. R. Gutierrez, M. Phan, and M. Batzill, "Strong room-temperature ferromagnetism in $\mathrm{VSe}_{2}$ monolayers on van der Waals substrates", Nat. Nanotechnol. 13, 289-294 (2018).

[76] Y. Deng, Y. Yu, Y. Song, J. Zhang, N. Z. Wang, Z. Sun, Y. Yi, Y. Z. Wu, S. Wu, J. Zhu, J. Wang, X. H. Chen, and Y. Zhang, "Gate-tunable room-temperature ferromagnetism in two-dimensional $\mathrm{Fe}_{3} \mathrm{GeTe}_{2}$ ", Nature (London) 563, 94-99 (2018).

[77] Though they state that they are studying trigonal prismatic $(\mathrm{H})$ monolayers, it is clear from their sketches of the structure as well as the density of states and magnetic moments that the calculations of Ma et al. were for the octahedral (T) phase [208]. Subsequent studies of both phases $[171 ; 209 ; 210 ; 211 ; 212 ; 213]$ find the $H$ phase to have a lower energy and to be magnetic with a moment of $1 \mu_{B} / \mathrm{V}$ atom. 
[78] T. Li and G. Galli, "Electronic properties of $\mathrm{MoS}_{2}$ nanoparticles", J. Phys. Chem. C 111, 16192-16196 (2007).

[79] R. A. Bromley, R. B. Murray, and A. D. Yoffe, "The band structures of some transition metal dichalcogenides: III. Group VI A: trigonal prism materials", J. Phys. C: Sol. State Phys. 5, 759-778 (1972).

[80] L. F. Mattheiss, "Band structures of transition-metal-dichalcogenide layer compounds", Phys. Rev. B 8, 3719-3740 (1973).

[81] C. Ataca and S. Ciraci, "Functionalization of single-layer $\mathrm{MoS}_{2}$ honeycomb structures", J. Phys. Chem. C 115, 13303-13311 (2011).

[82] S.-C. Lu and J.-P. Leburton, "Electronic structures of defects and magnetic impurities in $\mathrm{MoS}_{2}$ monolayers", Nanoscale Research Letters 9, 676 (2014).

[83] J. Hong, Z. Hu, M. Probert, K. Li, D. Lv, X. Yang, L. Gu, N. Mao, Q. Feng, L. Xie, J. Zhang, D. Wu, Z. Zhang, C. Jin, W. Ji, X. Zhang, J. Yuan, and Z. Zhang, "Exploring atomic defects in molybdenum disulphide monolayers", Nat. Commun. 6, 6293 (2015).

[84] W.-F. Li, C. Fang, and M. A. van Huis, "Strong spin-orbit splitting and magnetism of point defect states in monolayer WS 2 ", Phys. Rev. B 94, 195425 (2016).

[85] M. A. Khan and M. N. Leuenberger, "Room-temperature superparamagnetism due to giant magnetic anisotropy in $\mathrm{Mos}_{\mathrm{S}}$ defected single-layer $\mathrm{MoS}_{2}$ ", J. Phys.: Condens. Matter 30, 155802 (2018).

[86] J. He, K. Wu, R. Sa, Q. Li, and Y. Wei1, "Magnetic properties of nonmetal atoms absorbed $\mathrm{MoS}_{2}$ monolayers", Appl. Phys. Lett. 96, 082504 (2010).

[87] K. Dolui, I. Rungger, C. Pemmaraju, and S. Sanvito, "Possible doping strategies for $\mathrm{MoS}_{2}$ monolayers: An ab initio study", Phys. Rev. B 88, 075420 (2013).

[88] J. Karthikeyan, H. P. Komsa, M. Batzill, and A. V. Krasheninnikov, "Which transition metal atoms can be embedded into two-dimensional molybdenum dichalcogenides and add magnetism?", Nano Letters 19, 4581-4587 (2019).

[89] Y. C. Cheng, Z. Y. Zhu, W. B. Mi, Z. B. Guo, and U. Schwingenschlögl, "Prediction of two-dimensional diluted magnetic semiconductors: Doped monolayer $\mathrm{MoS}_{2}$ systems", Phys. Rev. B 87, 100401(R) (2013).

[90] Q. Yue, S. Chang, S. Qin, and J. Li, "Functionalization of monolayer $\mathrm{MoS}_{2}$ by substitutional doping: A first-principles study", Phys. Lett. A 377, 1362-1367 (2013).

[91] A. Ramasubramaniam and D. Naveh, "Mn-doped monolayer $\mathrm{MoS}_{2}$ : An atomically thin dilute magnetic semiconductor", Phys. Rev. B 87, 195201 (2013).

[92] R. Mishra, W. Zhou, S. J. Pennycook, S. T. Pantelides, and J. Idrobo, "Long-range ferromagnetic ordering in manganese-doped two-dimensional dichalcogenides", Phys. Rev. B 88, 144409 (2013). 
[93] W. Yun and J. D. Lee, "Unexpected strong magnetism of Cu doped single-layer $\mathrm{MoS}_{2}$ and its origin", Physical Chemistry Chemical Physics 16, 8990-8996 (2014).

[94] J. Qi, X. Li, X. Chen, and K. Hu, "Strain tuning of magnetism in Mn doped $\mathrm{MoS}_{2}$ monolayer", J. Phys.: Condens. Matter 26, 256003 (2014).

[95] C. J. Gil, A. Pham, A. Yu, and S. Li, "An ab initio study of transition metals doped with $\mathrm{WSe}_{2}$ for long-range room temperature ferromagnetism in two-dimensional transition metal dichalcogenide", J. Phys.: Condens. Matter 26, 306004 (2014).

[96] A. N. Andriotis and M. Menon, "Tunable magnetic properties of transition metal doped $\mathrm{MoS}_{2}$ ", Phys. Rev. B 90, 125304 (2014).

[97] J. Zhang, H. Zheng, R. Han, X. Du, and Y. Yan, "Tuning magnetic properties of $\mathrm{CrS}_{2}$ monolayer by doping transition metal and alkaline-earth atoms", J. Alloys Compd. 647, 75-81 (2015).

[98] Y. Miao, Y. Huang, Q. Fang, Z. Yang, K. Xu, F. Ma, and P. K. Chu, “Tuning of electronic states and magnetic polarization in monolayered $\mathrm{MoS}_{2}$ by codoping with transition metals and nonmetals", J. Mater. Sci. 51, 9514-9525 (2016).

[99] X. Zhao, C. Xia, T. Wang, and X. Dai, "Electronic and magnetic properties of X-doped (X = Ti, Zr, Hf) tungsten disulphide monolayer", J. Alloys Compd. 654, 574-579 (2016).

[100] X. Zhao, P. Chen, C. Xia, T. Wang, and X. Dai, "Electronic and magnetic properties of $n$-type and $p$-doped $\mathrm{MoS}_{2}$ monolayers", RSC Advances 6, 16772 (2016).

[101] X.-L. Fan, Y.-R. An, and W.-J. Guo, "Ferromagnetism in transitional metal-doped $\mathrm{MoS}_{2}$ monolayer", Nanoscale Research Letters 11, 154 (2016).

[102] A. W. Robertson, Y.-C. Lin, S. Wang, H. Sawada, C. S. Allen, Q. Chen, S. Lee, G.-D. Lee, J. Lee, S. Han, E. Yoon, A. I. Kirkland, H. Kim, K. Suenaga, and J. H. Warner, "Atomic structure and spectroscopy of single metal (Cr, V) substitutional dopants in monolayer $\mathrm{MoS}_{2}$ ", ACS Nano 10, 10227-10236 (2016).

[103] N. Singh and U. Schwingenschlögl, "A route to permanent valley polarization in monolayer $\mathrm{MoS}_{2}$ ”, Adv. Mater. 29, 1600970 (2017).

[104] Y. Miao, Y. Li, Q. Fang, Y. Huang, Y. Sund, K. Xub, F. Ma, and P. K. Chu, "Effects of dopant separation on electronic states and magnetism in monolayer $\mathrm{MoS}_{2}$ ", Appl. Surf. Sci. 428, 226-232 (2018).

[105] S. Mekonnen and P. Singh, "Electronic structure and nearly room-temperature ferromagnetism in V-doped monolayer and bilayer $\mathrm{MoS}_{2}$ ", International Journal of Modern Physics B 32, 1850231 (2018).

[106] Y. Gao, N. Ganguli, and P. J. Kelly, "Itinerant ferromagnetism in p-doped monolayers of $\mathrm{MoS}_{2}$ ”, Phys. Rev. B 99, 220406(R) (2019). 
[107] H. Peelaers and C. G. Van de Walle, "Effects of strain on band structure and effective masses in $\mathrm{MoS}_{2}$ ", Phys. Rev. B 86, 241401(R) (2012).

[108] D. M. Edwards and M. I. Katsnelson, "High-temperature ferromagnetism of sp electrons in narrow impurity bands: application to $\mathrm{CaB}_{6}$ ", J. Phys.: Condens. Matter 18, 7209-7225 (2006).

[109] P. E. Blöchl, "Projector augmented-wave method", Phys. Rev. B 50, 17953-17979 (1994).

[110] G. Kresse and J. Hafner, "Ab initio molecular dynamics for open-shell transition metals", Phys. Rev. B 48, 13115 (1993).

[111] G. Kresse and J. Furthmüller, "Efficient iterative schemes for $a b$ initio total-energy calculations using a plane-wave basis set", Phys. Rev. B 54, 11169-11186 (1996).

[112] G. Kresse and D. Joubert, "From ultrasoft pseudopotentials to the projector augmented-wave method", Phys. Rev. B 59, 1758-1775 (1999).

[113] T. Böker, R. Severin, A. Müller, C. Janowitz, R. Manzke, D. Voß, P. Krüger, A. Mazur, and J. Pollmann, "Band structure of $\mathrm{MoS}_{2}, \mathrm{MoSe}_{2}$, and a-MoTe 2 : Angle-resolved photoelectron spectroscopy and ab initio calculations", Phys. Rev. B 64, 235305 (2001).

[114] W. C. Jin, P. C. Yeh, N. Zaki, D. T. Zhang, J. T. Sadowski, A. Al-Mahboob, A. M. van der Zande, D. A. Chenet, J. I. Dadap, I. P. Herman, P. Sutter, J. Hone, and R. M. Osgood, "Direct Measurement of the Thickness-Dependent Electronic Band Structure of $\mathrm{MoS}_{2}$ using Angle-Resolved Photoemission Spectroscopy", Phys. Rev. Lett. 111, 106801 (2013).

[115] J. P. Perdew and A. Zunger, "Self-interaction correction to density-functional approximations for many-electron systems", Phys. Rev. B 23, 5048-5079 (1981).

[116] J. P. Perdew, K. Burke, and M. Ernzerhof, "Generalized gradient approximation made simple”, Phys. Rev. Lett. 77, 3865-3868 (1996).

[117] S. T. Pantelides, "The electronic structure of impurities and other point defects in semiconductors", Rev. Mod. Phys. 50, 797-858 (1978).

[118] M. Lannoo and J. Bourgoin, Point Defects in Semiconductors I: Theoretical Aspects, volume 22 of Springer Series in Solid-State Sciences (Springer, Berlin) (1981).

[119] M. Altarelli and F. Bassani, "Impurity states: Theoretical", in Handbook on Semiconductors edited by T. S. Moss, edited by W. Paul, volume 1, 269-322 (North-Holland, Amsterdam) (1982).

[120] J. S. Smith, A. Budi, M. C. Per, N. Vogt, D. W. Drumm, L. C. L. Hollenberg, J. H. Cole, and S. P. Russo, "Ab initio calculation of energy levels for phosphorus donors in silicon”, Scientific Reports 7, 6010 (2017). 
[121] O. Gunnarsson, B. Lundqvist, and J. W. Wilkins, "Contribution to the cohesive energy of simple metals: Spin-dependent effect”, Phys. Rev. B 10, 1319-1327 (1974).

[122] M. Aghajanian, A. A. Mostofi, and J. Lischner, "Tuning electronic properties of transition-metal dichalcogenides via defect charge”, Scientific Reports 8, 13611 (2018).

[123] X. L. Yang, S. H. Guo, F. T. Chan, K. W. Wong, and W. Y. Ching, "Analytic solution of a two-dimensional hydrogen atom. I. Nonrelativistic theory", Phys. Rev. A 43, 1186-1196 (1991).

[124] A. Laturia, M. L. Van de Put, and W. G. Vandenberghe, "Dielectric properties of hexagonal boron nitride and transition metal dichalcogenides: from monolayer to bulk", npj 2D Materials and Applications 2, 6 (2018).

[125] W. S. Yun, S. W. Han, S. C. Hong, I. G. Kim, and J. D. Lee, "Thickness and strain effects on electronic structures of transition metal dichalcogenides: $2 \mathrm{H}-\mathrm{MX}_{2}$ semiconductors ( $\mathrm{M}=\mathrm{Mo}, \mathrm{W}$; X = S, Se, Te)", Phys. Rev. B 85, 033305 (2012).

[126] O. Gunnarsson and B. Lundqvist, "Exchange and correlation in atoms, molecules, and solids by the spin-density-functional formalism", Phys. Rev. B 13, 4274-4298 (1976).

[127] J. F. Janak, "Uniform susceptibilities of metallic elements", Phys. Rev. B 16, 255-262 (1977).

[128] S. L. Dudarev, G. A. Botton, S. Y. Savrasov, C. J. Humphreys, and A. P. Sutton, "Electron-energy-loss spectra and the structural stability of nickel oxide: An LSDA+U study", Phys. Rev. B 57, 1505-1509 (1998).

[129] M. Wu, X. Yao, Y. Hao, H. Dong, Y. Cheng, H. Liu, F. Lu, W. Wang, K. Cho, and W.-H. Wang, "Electronic structures, magnetic properties and band alignments of 3d transition metal atoms doped monolayer $\mathrm{MoS}_{2}$ ", Phys. Lett. A 382, 111-115 (2018).

[130] L. Onsager, "Crystal Statistics. I. A Two-Dimensional Model with an Order-Disorder Transition", Phys. Rev. 65, 117-149 (1944).

[131] C. N. Yang, "The Spontaneous Magnetization of a Two-Dimensional Ising Model", Phys. Rev. 85, 808-816 (1952).

[132] A. R. Mackintosh and O. K. Andersen, "The electronic structure of transition metals", in Electrons at the Fermi Surface, edited by M. Springford, 149-224 (Cambridge University Press, Cambridge) (1980).

[133] V. Heine, "Electronic structure from the point of view of the local atomic environment", in Solid State Physics, edited by H. Ehrenreich, F. Seitz, and D. Turnbull, volume 35, 1-123 (Academic Press, New York) (1980).

[134] G. H. O. Daalderop, P. J. Kelly, and M. F. H. Schuurmans, "First-principles calculation of the magnetocrystalline anisotropy energy of iron, cobalt and nickel", Phys. Rev. B 41, 11919-11937 (1990). 
[135] M. D. Stiles, S. V. Halilov, R. A. Hyman, and A. Zangwill, "Spin-other-orbit interaction and magnetocrystalline anisotropy", Phys. Rev. B 64, 104430 (2001).

[136] G. H. O. Daalderop, P. J. Kelly, and M. F. H. Schuurmans, "Magnetic anisotropy of a free-standing Co monolayer and of multilayers which contain Co monolayers", Phys. Rev. B 50, 9989-10003 (1994).

[137] G. H. O. Daalderop, P. J. Kelly, and M. F. H. Schuurmans, "Magnetic anisotropy from first principles", in Ultrathin Magnetic Structures I, edited by J. A. C. Bland and B. Heinrich, 40-64 (Springer-Verlag, Berlin Heidelberg) (1994).

[138] Z. Y. Zhu, Y. C. Cheng, and U. Schwingenschlögl, "Giant spin-orbit-induced spin splitting in two-dimensional transition-metal dichalcogenide semiconductors", Phys. Rev. B 84, 153402 (2011).

[139] G. H. O. Daalderop, P. J. Kelly, and F. J. A. den Broeder, "Prediction and confirmation of perpendicular magnetic anisotropy in Co/Ni multilayers", Phys. Rev. Lett. 68, 682-685 (1992).

[140] P. E. Blöchl, O. Jepsen, and O. K. Andersen, "Improved tetrahedron method for Brillouin-zone integrations", Phys. Rev. B 49, 16223-16233 (1994).

[141] C. Xu, J. Feng, H. Xiang, and L. Bellaiche, "Interplay between Kitaev interaction and single ion anisotropy in ferromagnetic $\mathrm{CrI}_{3}$ and $\mathrm{CrGeTe}_{3}$ monolayers", npj Computational Materials 4, 57 (2018).

[142] S. A. Leonel, A. Oliveira, B. V. Costa, and P. Coura, "Comparative study between a two-dimensional anisotropic Heisenberg antiferromagnet with easy-axis single-ion anisotropy and one with easy-axis exchange anisotropy", J. Magn. Magn. Mater. 305, 157-164 (2006).

[143] K. Binder, "Finite size scaling analysis of Ising model block distribution functions", Z. Phys. B 43, 119-140 (1981).

[144] D. P. Landau and K. Binder, A Guide to Monte Carlo Simulations in Statistical Physics, 3 edition (Cambridge University Press, Cambridge, U.K.) (2009).

[145] D. Vanderbilt, "Soft self-consistent pseudopotentials in a generalized eigenvalue formalism", Phys. Rev. B 41, 7892-7895 (1990).

[146] N. Troullier and J. L. Martins, "Efficient pseudopotentials for plane-wave calculations", Phys. Rev. B 43, 1993-2006 (1991).

[147] D. M. Ceperley and B. J. Alder, "Ground state of the electron gas by a stochastic method", Phys. Rev. Lett. 45, 566-569 (1980).

[148] J. Heyd, G. E. Scuseria, and M. Ernzerhof, "Hybrid functionals based on a screened coulomb potential", J. Chem. Phys. 118, 8207-8215 (2003). 
[149] P. Giannozzi, S. Baroni, N. Bonini, M. Calandra, R. Car, C. Cavazzoni, D. Ceresoli, G. L. Chiarotti, M. Cococcioni, I. Dabo, A. Dal Corso, S. Fabris, G. Fratesi, S. de Gironcoli, R. Gebauer, U. Gerstmann, C. Gougoussis, A. Kokalj, M. Lazzeri, L. MartinSamos, N. Marzari, F. Mauri, R. Mazzarello, S. Paolini, A. Pasquarello, L. Paulatto, C. Sbraccia, S. Scandolo, G. Sclauzero, A. P. Seitsonen, A. Smogunov, P. Umari, and R. M. Wentzcovitch, "QUANTUM ESPRESSO: a modular and open-source software project for quantum simulations of materials", J. Phys.: Condens. Matter 21, 395502 (2009).

[150] P. Blaha, K. Schwarz, and G. K. H. Madsen, "Electronic structure calculations of solids using the WIEN2k package for material sciences", Comput. Phys. Commun. 147, 71-76 (2002).

[151] J. M. Soler, E. Artacho, J. D. Gale, A. García, J. Junquera, P. Ordejón, and D. SánchezPortal, "The siesta method for ab initio order-n materials simulation", J. Phys.: Condens. Matter 14, 2745-2779 (2002).

[152] M. Choi, "Strain-enhanced $p$ doping in monolayer $\mathrm{MoS}_{2}$ ", Phys. Rev. Appl. 9, 024009 (2018).

[153] S. Yun, D. Duong, M. Doan, K. Singh, T. Phan, W. Choi, Y. Kim, and Y. Lee, "Room-temperature ferromagnetism in monolayer $\mathrm{WSe}_{2}$ semiconductor via vanadium dopant", arXiv:1806.06479 .

[154] Z. Guguchia, A. Kerelsky, D. Edelberg, S. Banerjee, F. von Rohr, D. Scullion, M. Augustin, M. Scully, D. A. Rhodes, Z. Shermadini, H. Luetkens, A. Shengelaya, C. Baines, E. Morenzoni, A. Amato, J. C. Hone, R. Khasanov, S. J. L. Billinge, E. Santos, A. N. Pasupathy, and Y. J. Uemura, "Magnetism in semiconducting molybdenum dichalcogenides", Science Advances 4, eeat3672 (2018).

[155] P. M. Coelho, H.-P. Komsa, K. Lasek, V. Kalappattil, J. Karthikeyan, M.-H. Phan, A. V. Krasheninnikov, and M. Batzill, "Room-temperature ferromagnetism in $\mathrm{MoTe}_{2}$ by post-growth incorporation of vanadium impurities", Advanced Electronic Materials 5, 1900044 (2019).

[156] L. Yang, H. Wu, W. Zhang, X. Lou, Z. Xie, X. Yu, Y. Liu, and H. Chang, "Ta Doping Enhanced Room-Temperature Ferromagnetism in 2D Semiconducting $\mathrm{MoTe}_{2}$ Nanosheets", Advanced Electronic Materials 5, 1900552 (2019).

[157] W. Hu, H. Tan, H. Duan, G. Li, N. Li, Q. Ji, Y. Lu, Y. Wang, Z. Sun, F. Hu, C. Wang, and W. Yan, "Synergetic Effect of Substitutional Dopants and Sulfur Vacancy in Modulating the Ferromagnetism of $\mathrm{MoS}_{2}$ Nanosheets", ACS Applied Materials \& Interfaces 11, 31155-31161 (2019).

[158] Y. Gao, N. Ganguli, and P. J. Kelly, "DFT study of itinerant ferromagnetism in p-doped monolayers of $\mathrm{MoS}_{2}$ ", Phys. Rev. B 100, 235440 (2019).

[159] U. von Barth and L. Hedin, "A local exchange-correlation potential for the spinpolarized case: I", J. Phys. C: Sol. State Phys. 5, 1629-1642 (1972). 
[160] O. Gunnarsson, "Band model for magnetism of transition metals in the spin-densityfunctional formalism”, J. Phys. F: Met. Phys. 6, 587-606 (1976).

[161] O. K. Andersen, J. Madsen, U. K. Poulsen, O. Jepsen, and J. Kollar, "Magnetic groundstate properties of transition-metals", Physica B+C 86, 249-256 (1977).

[162] K. D. Bronsema, J. L. D. Boer, and F. Jellinek, "On the structure of molybdenum diselenide and disulfide", Z. Anorg. Allg. Chem 540, 15 (1986).

[163] Y. Zhang, T. Chang, B. Zhou, Y. Cui, H. Yan, Z. Liu, F. Schmitt, J. Lee, R. Moore, Y. Chen, H. Lin, H.-T. Jeng, S.-K. Mo, Z. Hussain, A. Bansil, and Z. Shen, "Direct observation of the transition from indirect to direct bandgap in atomically thin epitaxial $\mathrm{MoSe}_{2}$ ", Nat. Nanotechnol. 9, 111-115 (2014).

[164] N. R. Wilson, P. V. Nguyen, K. Seyler, P. Rivera, A. J. Marsden, Z. P. L. Laker, G. C. Constantinescu, V. Kandyba, A. Barinov, N. D. M. Hine, X. Xu, and D. H. Cobden, "Determination of band offsets, hybridization, and exciton binding in 2D semiconductor heterostructures", Science Advances 3, e1601832 (2017).

[165] O. Knop and R. D. MacDonald, "Chalcogenides of the transition elements: III. Molybdenum ditelluride”, Can. J. Chem. 39, 897-904 (1961).

[166] C. Ruppert, O. B. Aslan, and T. F. Heinz, "Optical Properties and Band Gap of Singleand Few-Layer MoTe ${ }_{2}$ Crystals", Nano Letters 14, 6231-6236 (2014).

[167] W. J. Schutte, J. L. de Boer, and F. Jellinek, "Crystal structures of tungsten disulfide and diselenide", Can. J. Chem. 70, 207-209 (1987).

[168] S. Tongay, W. Fan, J. Kang, J. Park, U. Koldemir, J. Suh, D. S. Narang, K. Liu, J. Ji, J. Li, R. Sinclair, and J. Wu, "Tuning interlayer coupling in large-area heterostructures with CVD grown $\mathrm{MoS}_{2}$ and $\mathrm{WS}_{2}$ monolayers", Nano Letters 14, 3185-3190 (2014).

[169] C. Kastl, C. T. Chen, R. J. Koch, B. Schuler, T. R. Kuykendall, A. Bostwick, C. Jozwiak, T. Seyller, E. Rotenberg, A. Weber-Bargioni, S. Aloni, and A. M. Schwartzberg, "Multimodal spectromicroscopy of monolayer $\mathrm{WS}_{2}$ enabled by ultra-clean van der Waals epitaxy", 2D Materials 5, 045010 (2018).

[170] W.-T. Hsu, L.-S. Lu, D. Wang, J.-K. Huang, M.-Y. Li, T.-R. Chang, Y.-C. Chou, Z.-Y. Juang, H.-T. Jeng, L.-J. Li, and W.-H. Chang, "Evidence of indirect gap in monolayer $\mathrm{WSe}_{2}$ ", Nat. Commun. 8, 929 (2017).

[171] C. Ataca, H. Şahin, and S. Ciraci, "Stable, Single-Layer $\mathrm{MX}_{2}$ Transition-Metal Oxides and Dichalcogenides in a Honeycomb-Like Structure", J. Phys. Chem. C 116, 89838999 (2012).

[172] H. L. Zhuang, M. D. Johannes, M. N. Blonsky, and R. G. Hennig, "Computational prediction and characterization of single-layer $\mathrm{CrS}_{2}$ ", Appl. Phys. Lett. 104, 022116 (2014).

[173] C. F. van Bruggen, R. J. Haange, G. A. Wiegers, and D. K. G. de Boer, " $\mathrm{CrSe}_{2}$, a new layered dichalcogenide", Physica B+C 99, 166-172 (1980). 
[174] C. Wang, X. Zhou, Y. Pan, J. Qiao, X. Kong, C.-C. Kaun, and W. Ji, "Layer and doping tunable ferromagnetic order in two-dimensional $\mathrm{CrS}_{2}$ layers", Phys. Rev. B 97, 245409 (2018).

[175] Y. Qu, H. Pan, and C. Kwok, "Hydrogenation-controlled phase transition on twodimensional transition metal dichalcogenides and their unique physical and catalytic properties", Scientific Reports 6, 34186 (2016).

[176] J. A. Wilson and A. D. Yoffe, "Transition metal dichalcogenides discussion and interpretation of observed optical, electrical and structural properties", Adv. Phys. 18, 193-335 (1969).

[177] In the simplest case, $x$ is the symmetry-lowering displacement of a single atom. More generally, it represents a "collective coordinate" describing some more complex distortion of the central and surrounding atoms.

[178] H. A. Jahn and E. Teller, "Stability of polyatomic molecules in degenerate electronic states. I. Orbital degeneracy”, Proc. Roy. Soc. A 161, 220-235 (1937).

[179] K. H. Michel, D. Cakir, C. Sevik, and F. M. Peeters, "Piezoelectricity in twodimensional materials: Comparative study between lattice dynamics and ab initio calculations", Phys. Rev. B 95, 125415 (2017).

[180] Y. Toyozawa, "Phonon structures in the spectra of solids", Journal of Luminescence 1, 732-746 (1970).

[181] Z. Fei, B. Huang, P. Malinowski, W. Wang, T. Song, J. Sanchez, W. Yao, D. Xiao, D. H. Cobden, J.-H. Chu, and X. Xu, "Two-dimensional itinerant ferromagnetism in atomically thin $\mathrm{Fe}_{3} \mathrm{GeTe}_{2}$ ", Nat. Mater. 17, 778-782 (2018).

[182] P. K. J. Wong, W. Zhang, F. Bussolotti, X. Yin, T. S. Herng, L. Zhang, Y. L. Huang, G. Vinai, Y. J. Zheng, R. K. E. J. Goh, J. Ding, M.-T. Lin, G. Brocks, M. P. de Jong, A. H. C. Neto, and A. T. S. We, "Evidence of spin frustration in a vanadium diselenide monolayer magnet”, Adv. Mater. 31, 1901185 (2019).

[183] W. Zhang, P. K. J. Wong, X. Zhou, A. Rath, Z. Huang, H. Wang, S. A. Morton, J. Yuan, L. Zhang, R. Chua, S. Zeng, E. Liu, F. Xu, Ariando, D. H. C. Chua, Y. P. Feng, G. van der Laan, S. J. Pennycook, Y. Zhai, and A. T. S. Wee, "Ferromagnet/twodimensional semiconducting transition-metal dichalcogenide interface with perpendicular magnetic anisotropy", ACS Nano 13, 2253-2261 (2019).

[184] W. Zhang, L. Zhang, P. K. J. Wong, J. Yuan, G. Vinai, P. Torelli, G. van der Laan, Y. P. Feng, and A. T. S. Wee, "Magnetic transition in monolayer $\mathrm{VSe}_{2}$ via interface hybridization”, ACS Nano 13, 8897-9004 (2019).

[185] S. Wang, A. Robertson, and J. H. Warner, "Exploring atomic defects in molybdenum disulphide monolayers", Chem. Soc. Rev. 47, 6764 (2018).

[186] H. I. Rasool, C. Ophus, and A. Zettl, "Atomic defects in two dimensional materials", Adv. Mater. 27, 5771-5777 (2015). 
[187] H.-P. Komsa and A. V. Krasheninnikov, "Native defects in bulk and monolayer $\mathrm{MoS}_{2}$ from first principles", Phys. Rev. B 91, 125304 (2015).

[188] S. Haldar, H. Vovusha, M. K. Yadav, O. Eriksson, and B. Sanyal, "A systematic study of structural, electronic and optical properties of atomic scale defects in twodimensional transition metal dichalcogenides $\mathrm{MX}_{2}(\mathrm{M}=\mathrm{Mo}, \mathrm{W} ; \mathrm{X}=\mathrm{S}, \mathrm{Se}, \mathrm{Te})$ ", Phys. Rev. B 92, 235408 (2015).

[189] S.-Z. Yang, W. Sun, Y.-Y. Zhang, Y. Gong, M. P. Oxley, A. R. Lupini, P. M. Ajayan, M. F. Chisholm, S. T. Pantelides, and W. Zhou, "Direct Cation Exchange in Monolayer $\mathrm{MoS}_{2}$ via Recombination-Enhanced Migration”, Phys. Rev. Lett. 122, 106101 (2019).

[190] S. C. Erwin and F. Himpsel, "Intrinsic magnetism at silicon surfaces", Nat. Commun. 1, 58 (2010).

[191] M. Ferri, G. Fratesi, G. Onida, and A. Debernardi, "Ab initio study of the structural, electronic, magnetic, and optical properties of silicene nanoribbons", Phys. Rev. B 99, 085414 (2019).

[192] A. Hattori, S. Tanaya, K. Yada, M. Araidai, M. Sato, Y. Hatsugai, K. Shiraishi, and Y. Tanaka, "Edge states of hydrogen terminated monolayer materials: silicene, germanene and stanene ribbons", J. Phys.: Condens. Matter 29, 115302 (2017).

[193] B. van den Broek, M. Houssa, A. Lu1, G. Pourtois, V. Afanas'ev, and A. Stesmans, "Silicene nanoribbons on transition metal dichalcogenide substrates: Effects on electronic structure and ballistic transport", Nanoscale Research 9(11), 3394-3406 (2016).

[194] Y.-L. Song, S. Zhang, D.-B. Lu, H. ru Xu, Z. Wang, Y. Zhang, and Z.-W. Lu, "Bandgap modulations of armchair silicene nanoribbons by transverse electric fields", Eur. Phys. J. B 86, 488 (2013).

[195] S. Rachel and M. Ezawa, "Giant magnetoresistance and perfect spin filter in silicene, germanene, and stanene”, Phys. Rev. B 89, 195303 (2014).

[196] J. C. Meyer, C. Kisielowski, R. Erni, M. D. Rossell, M. F. Crommie, and A. Zettl, "Direct imaging of lattice atoms and topological defects in graphene membranes", 8(11), 3582-3586 (2008).

[197] K. He, A. W. Robertson, S. Lee, E. Yoon, G.-D. Lee, and J. H. Warner, "Extended klein edges in graphene", ACS Nano 8(12), 12272-12279 (2014).

[198] Y.-L. Song, Y. Zhang, J.-M. Zhang, and D.-B. Lu, "Effects of the edge shape and the width on the structural and electronicproperties of silicene nanoribbons", Appl. Surf. Sci. 256, 6313-6317 (2010).

[199] J. N. B. Rodrigues, P. A. D. Gonçalves, N. F. G. Rodrigues, R. M. Ribeiro, J. M. B. L. dos Santos, and N. M. R. Peres, "Zigzag graphene nanoribbon edge reconstruction with stone-wales defects”, Phys. Rev. B 84, 155435 (2011). 
[200] Y.-W. Son, M. L. Cohen, and S. G. Louie, "Half-metallic graphene nanoribbons", Nature (London) 444, 347-349 (2006).

[201] Y.-W. Son, M. L. Cohen, and S. G. Louie, "Energy gaps in graphene nanoribbons", Phys. Rev. Lett. 97, 216803 (2006).

[202] A. Acun, L. Zhang, P. Bampoulis, M. Farmanbar, A. van Houselt, A. N. Rudenko, M. Lingenfelder, G. Brocks, B. Poelsema, M. I. Katsnelson, and H. J. W. Zandvliet, "Germanene: the germanium analogue of graphene", J. Phys.: Condens. Matter 27, 443002 (2015).

[203] C. Cahangirov, M. Topsakal, E. Akturk, H. Sahin, and S. Ciraci, "Two- and onedimensional honeycomb structures of silicon and germanium", Phys. Rev. Lett. 102, 236804 (2009).

[204] R. Li, J. Zhou, Y. Han, J. Dong, and Y. Kawazoe, "A new $(2 \times 1)$ reconstructed edge structure of zigzag si nanoribbon: First principles study", J. Chem. Phys. 139, 104703 (2013).

[205] L. Zhang, P. Bampoulis, A. van Houselt, and H. J. W. Zandvliet, "Two-dimensional dirac signature of germanene", Appl. Phys. Lett. 107, 111605 (2015).

[206] A. Molle, J. Goldberger, M. Houssa, Y. Xu, S.-C. Zhang, and D. Akinwande, "Buckled two-dimensional xene sheets", Nat. Mater. 16, 163-169 (2017).

[207] A. Ramstad, G. Brocks, and P. J. Kelly, "Theoretical study of the Si(100) surface reconstruction", Phys. Rev. B 51, 14504 (1995).

[208] Y. Ma, Y. Dai, M. Guo, C. Niu, Y. Zhu, and B. Huang, "Evidence of the existence of magnetism in pristine $\mathrm{VX}_{2}$ monolayers $(\mathrm{X}=\mathrm{S}$, Se) and their strain-induced tunable magnetic properties", ACS Nano 6, 1695-1701 (2012).

[209] H. Zhang, L.-M. Liu, and W.-M. Lau, "Dimension-dependent phase transition and magnetic properties of $\mathrm{VS}_{2}$ ", Journal of Materials Chemistry A 1, 10821-10828 (2013).

[210] L.-Y. Gan, Q. Zhang, Y. Cheng, and U. Schwingenschlögl, "Two-dimensional ferromagnet/semiconductor transition metal dichalcogenide contacts: $p$-type Schottky barrier and spin-injection control", Phys. Rev. B 88, 235310 (2013).

[211] M. Kan, B. Wang, Y. H. Lee, and Q. Sun, "A density functional theory study of the tunable structure, magnetism and metal-insulator phase transition in $\mathrm{VS}_{2}$ monolayers induced by in-plane biaxial strain", Nano Research 8, 1348-1356 (2015).

[212] A. H. M. A. Wasey, S. Chakrabarty, and G. P. Das, "Quantum size effects in layered $\mathrm{VX}_{2}(\mathrm{X}=\mathrm{S}, \mathrm{Se})$ materials: Manifestation of metal to semimetal or semiconductor transition", J. Appl. Phys. 117, 064313 (2015).

[213] H. L. Zhuang and R. G. Hennig, "Stability and magnetism of strongly correlated single-layer VS 2 ”, Phys. Rev. B 93, 054429 (2016). 


\section{List of publications}

1 Itinerant ferromagnetism in p-doped monolayers of $\mathrm{MoS}_{2}$

Y. Gao, N. Ganguli, P. J. Kelly,

Phys. Rev. B 99, 220406(R), 2019.

2 DFT study of itinerant ferromagnetism in p-doped monolayers of $\mathrm{MoS}_{2}$

Y. Gao, N. Ganguli, P. J. Kelly

Phys. Rev. B 100, 235440, 2019.

3 The ferroelectric field effect on the two-dimensional electron gas at $\mathrm{LaAlO}_{3} / \mathrm{SrTiO}_{3}$ (001) interface: Insights from first principle simulations

Y. Gao, P. Li, N. Wang, H. Xing, C. Chen

Physica E: Low-dimensional Systems and Nanostructures, 80: 195-201,2016.

4 Defect Engineering of $\mathrm{MnO}_{2}$ Nanosheets by Substitutional Doping for Printable Solid-State Micro-Supercapacitors

Y. Wang, Y. Zhang, Y. Gao, G. Sheng, J. E. ten Elshof

Nano Energy 104306,2019.

5 Photocarrier transport and dynamics in mixed-phase BiFeO3 films

P. Li, X. Dong, Y. Gao, L. Ren, K. Jin

Optics Express 24 (8), 9119-9129,2016. 


\section{Summary}

The first isolation of graphene in 2004 opened a new area of 2D Van der Waals materials with one or several elements arranged in one plane with atomic thin thickness, which attracts great attention for the potential in the miniaturization of electronic devices. Various spectacular properties that do not exist in their 3D bulk form also provide opportunities for the design of novel electronic devices. In this thesis, by first principle calculations, I investigate the electronic properties of several typical 2D systems including the $\mathrm{LaAlO}_{3} / \mathrm{SrTiO}_{3}$ (LAO/STO) (001) n-type interface, monolayer of transition metal dichalcogenides (TMDs) and 2D-Xene ( $\mathrm{X}=\mathrm{Si}, \mathrm{Ge}, \mathrm{Sn}$ ) and explore the possibility to tune them for practical application.

In chapter 2, I tune the two-dimensional electron gas at the n-type LAO/STO (001) interface through ferroelectric substrate $\mathrm{BaTiO}_{3}$ (BTO). First, I evaluate the carrier density at the interface to show the existence of polar distortions in LAO and STO. Then the BTO substrate is polarized perpendicular to the interface and it is found that the carrier density at the LAO/STO interface can be reversely tuned by the ferroelectric field of the substrate accompanied with a metal to insulator transition. Through potential analysis, we further confirm that the ferroelectric field control of the intrinsic electric field and therefore the electronic reconstructions at the interface originate from the screening of polarization charges.

In chapter 3, I explore the possibility of realizing long-range ferromagnetism in $\mathrm{MoS}_{2}$ monolayer by substitutionally doped single acceptor (V,Nb,Ta). Using the periodic supercell approach, we identify three impurity levels originating from $K / K^{\prime}$ and $\Gamma$ valley and get the dependence of their binding energy on the supercell size. With a simple hydrogen model, the effective Bohr radius and dielectric constant are extracted by fitting the impurity Coulomb potential curve. The exchange interaction between dopants are evaluated by calculating the energy difference between spin antiparallel alignment (AFM) and parallel alignment (FM). Taking account of spin orbit coupling, a formula is derived to determine the single ion anisotropy in V-doped monolayer of $\mathrm{MoS}_{2}$. With the extracted exchange parameter, I perform Monte Carlo calculations by using Binder's cumulant method to evaluate the Curie temperature for V-doped monolayer of $\mathrm{MoS}_{2}$. A Curie temperature of $165 \mathrm{~K}$ is found at $9 \% \mathrm{~V}$-doped $\mathrm{MoS}_{2}$ monolayer. The magnetic moment is found to be quenched by the formation of strong $\pi$ bond as the dopants pair is close enough, which limits the Curie temperature. Finally, possible solutions to avoid the quenching are proposed 
by considering other TMD system such as monolayers of $\mathrm{MoSe}_{2}$ or $\mathrm{MoTe}_{2}$.

In chapter 4, I do a systematic study of the structural and electronic properties of single acceptor and double acceptor $(\mathrm{V}, \mathrm{Nb}, \mathrm{Ta}, \mathrm{Ti}, \mathrm{Zr}, \mathrm{Hf})$ doped $\mathrm{MX}_{2}$ monolayers to find the general trend of the impurity behaviour. I first examine the bulk properties of $\mathrm{MX}_{2}$ monolayers, which determines the behaviour of the impurity levels bound to their band edges. The binding energy, formation energy, Jahn-Teller distortion, and single ion anisotropy of the single and double acceptors in $\mathrm{MX}_{2}$ monolayers are systematically calculated in single doping limit. In systems with double acceptors, the Jahn-Teller distortion competes with exchange splitting and may quench the magnetic moment as it is strong enough. In addition, double acceptors give rise to an in-plane single ion anisotropy. In systems with single acceptors, the Jahn-Teller distortion is detrimental to the long-range exchange interaction. In the end, possible effects of intrinsic defects on the dopant and systems promising for realizing the room temperature ferromagnetism are discussed.

In chapter 5 , the zigzag edge reconstructions of $2 \mathrm{D}-\mathrm{Xene}(\mathrm{X}=\mathrm{Si}, \mathrm{Ge}, \mathrm{Sn})$ nano ribbons are investigated by first principles calculations. Different size of unit cells of zigzag nanoribbon $9 \times n(n=1,2,3,4,6,8)$ are constructed to include all possible edge reconstructions. Three different kinds of edge reconstructions $(2 a, 3 a, 4 a)$ are found. By comparing the edge energies of each system, edge reconstruction with $3 a$ periodicity has the lowest edge energy and opens a gap at the Fermi level. The band filling of the dangling states near the Fermi level associated with the buckling is found to be the main factor that determines the periodicity of the edge reconstruction. Furthermore, the wavefunction resulting from DFT calculations are projected on wannier function basis and the hopping parameters between different orbitals are obtained. The buckling of the atomic lattice determines the hopping between $s p^{2}$ and $p_{z}$ orbitals and so the bonding interaction. 


\section{Acknowledgements}

I want to give a big thank to the people who have helped me. Without the help of all those people, I can not make it possible.

First of all, I would give my sincerely gratitude to my daily supervisor Paul J. Kelly. Thanks for giving me the chance to study in the group and keep funding me to finish my Ph.D. You are always so patient and kind to teach me everything from the beginning. I am surprised by your insight into the results I present to you. I admire your critical attitude to the work that a scientist should have. The devil is in the detail. You told me " Do it quickly, then you would not do it perfectly". I really enjoy and appreciate the discussion we had in your office. Your positive attitude and curiosity to science inspire me to finish my Ph.D. Thanks for reviewing my paper and thesis, it must take you lots of time and efforts.

My colleagues in the group, the CMSer, thanks for all of your help and support in my Ph.D. Geert, I enjoy the talk with you about history, culture, society, and tradition. I learned a lot about Europe from you. We worked together in the "2D material" project. You always can give a clear and simple explanation to the problem and make a joke by the way. Els, thanks for your secretary work and you did a great job. All the best for your retired life. Sridevi, you told me how to run the job on our cluster and lots of other thing to live in Enschede when I came here. We share the office and work on similar project. I really enjoy the discussion we had on the physics problem and also about things happened in China and India. Thanks for all of your help and all the best for your thesis. Rohit, I remember the workshop we did together in Leiden. You are our main force and did most of the work. You never complain about it. You help me to do the tutorial which I should share with you. A big thank to you. Kriti, thanks for your kind help on many thing. Every time I walked into your office to ask for help, you always give me very detailed information. Your kindly smile always warm others. All the bests for your work. Ensan, I enjoy the lunch talk. We share many things about life. Wish you have a bright future.

I want to express my thanks to all of the friends I met in Netherlands. Bingbing Zhang, thanks for accommodating me when I came here. Even though just four months, it is quite a pleasure to be roommate with you. And Nico, you are so kind and I really enjoy the discussion with you. Hope you are healthy and happy. Harold, thanks for sharing your experimental data with us, I admire your hard 
working and curiosity to science, you are like a good friend to the students. Bing $\mathrm{Hu}$, thanks for the dinners in your home. I really like the Saturday night when we play cards together. We have lots of memories in the trip to the Belgium. Wish you and your wife have a bright future. Zhiyuan, my roommate, we cycled in Enschede and Gronau. That is my most relaxed time. And all of the other friends in Twente, thanks for your help.

Mom and Dad, you are my spiritual pillar that make me to finish my Ph.D. You give me freedom to do what I want to and support me whenever I need. Thanks for all you have done for the family. I would spend more time with you after I finish my Ph.D. My elder brother, Yudong, thanks for taking care of our parents when I am not at home.

At last, I would give my deepest appreciation to my wife, Pan. You came to Netherlands for me and started your life from the beginning. You managed to take care of yourself, especially during the period of your pregnancy. Thanks for all of the patience and encourage during the last year of my Ph.D. My unborn daughter, though I do not have a name for you yet, thanks for coming this year, we are looking forward to your coming.

Feb. 2020, Enschede 
ISBN: 978-90-365-5005-5

DOI: 10.3990/1.9789036550055 